NATIONAL LABORATORY

MANAGED BY UT-BATTELLE

FOR THE DEPARTMENT OF ENERGY

\title{
TSUNAMI Primer: A Primer for Sensitivity/Uncertainty Calculations with SCALE
}

\section{January 2009}

Prepared by

B. T. Rearden

D. E. Mueller

S. M. Bowman

R. D. Busch

S. J. Emerson

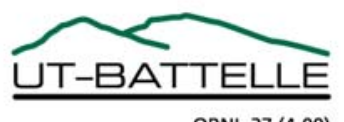

ORNL-27 (4-00) 


\section{DOCUMENT AVAILABILITY}

Reports produced after January 1, 1996, are generally available free via the U.S. Department of Energy (DOE) Information Bridge.

Web site http://www.osti.gov/bridge

Reports produced before January 1, 1996, may be purchased by members of the public from the following source.

National Technical Information Service

5285 Port Royal Road

Springfield, VA 22161

Telephone 703-605-6000 (1-800-553-6847)

TDD 703-487-4639

Fax 703-605-6900

E-mail info@ntis.gov

Web site http://www.ntis.gov/support/ordernowabout.htm

Reports are available to DOE employees, DOE contractors, Energy Technology Data Exchange (ETDE) representatives, and International Nuclear Information System (INIS) representatives from the following source.

Office of Scientific and Technical Information

P.O. Box 62

Oak Ridge, TN 37831

Telephone 865-576-8401

Fax 865-576-5728

E-mail reports@osti.gov

Web site http://www.osti.gov/contact.html

This report was prepared as an account of work sponsored by an agency of the United States Government. Neither the United States Government nor any agency thereof, nor any of their employees, makes any warranty, express or implied, or assumes any legal liability or responsibility for the accuracy, completeness, or usefulness of any information, apparatus, product, or process disclosed, or represents that its use would not infringe privately owned rights. Reference herein to any specific commercial product, process, or service by trade name, trademark, manufacturer, or otherwise, does not necessarily constitute or imply its endorsement, recommendation, or favoring by the United States Government or any agency thereof. The views and opinions of authors expressed herein do not necessarily state or reflect those of the United States Government or any agency thereof. 
Nuclear Science and Technology Division

\title{
TSUNAMI Primer: A Primer for Sensitivity/Uncertainty Calculations with SCALE
}

B. T. Rearden

D. E. Mueller

S. M. Bowman

R. D. Busch (University of New Mexico)

S. J. Emerson (University of New Mexico)

Date Published: January 2009

\author{
Prepared by \\ OAK RIDGE NATIONAL LABORATORY \\ P.O. Box 2008 \\ Oak Ridge, Tennessee 37831-6285 \\ managed by \\ UT-BATTELLE, LLC \\ for the \\ U.S. DEPARTMENT OF ENERGY \\ under contract DE-AC05-00OR22725
}





\section{CONTENTS}

\section{Page}

LIST OF FIGURES

$\mathrm{v}$

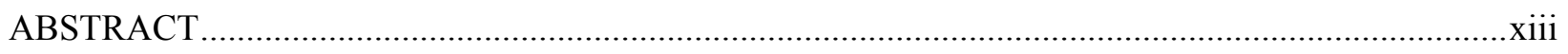

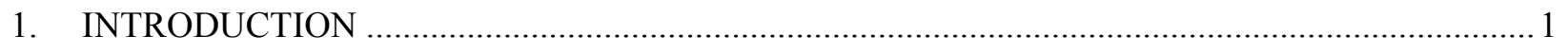

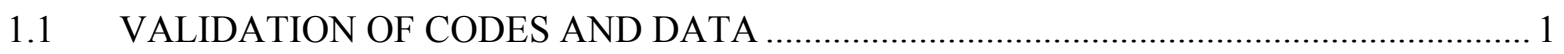

1.2 TSUNAMI TECHNIQUES FOR CODE VALIDATION .............................................. 2

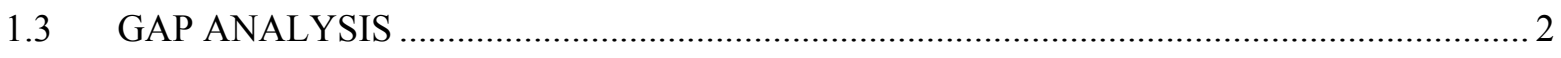

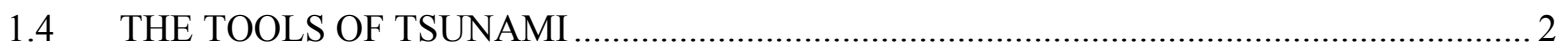

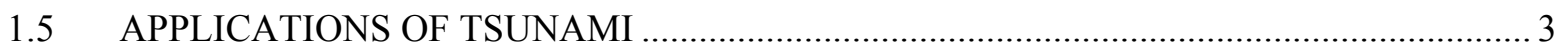

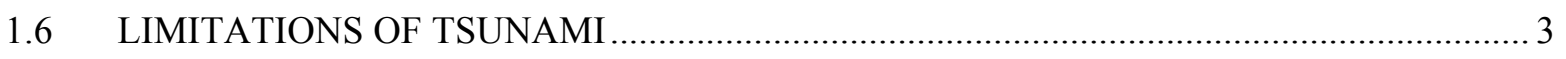

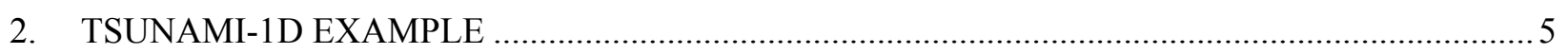

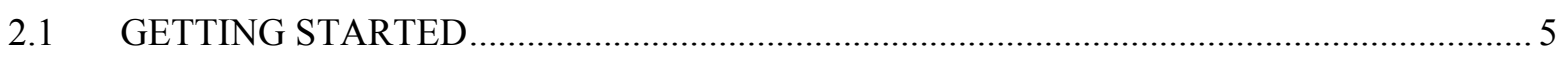

2.2 QUICK START EXAMPLE ...................................................................................... 5

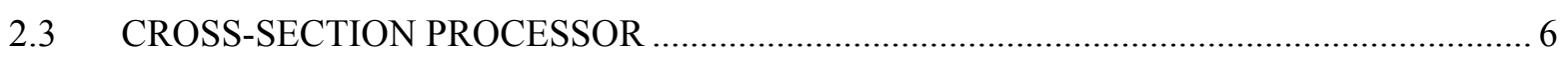

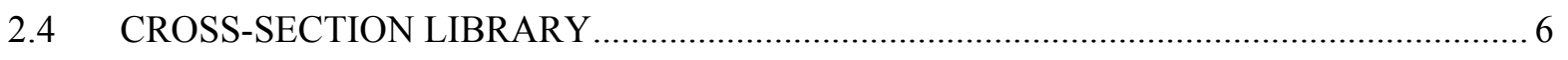

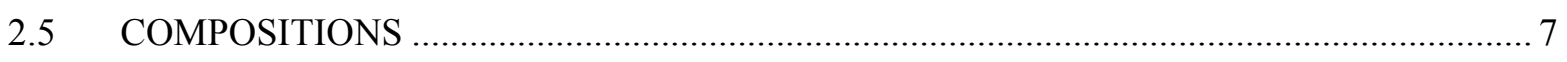

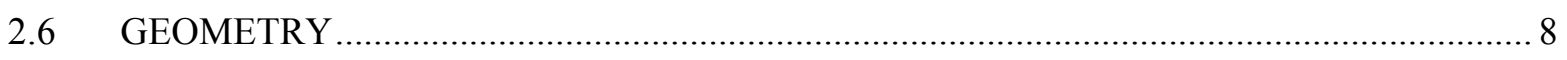

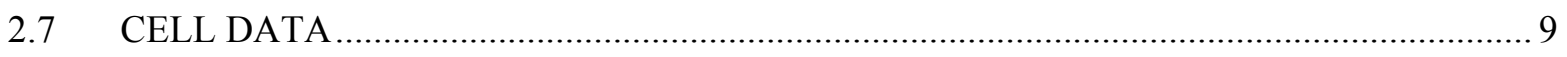

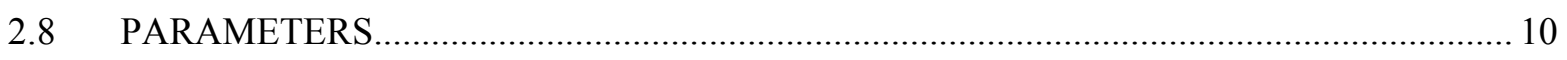

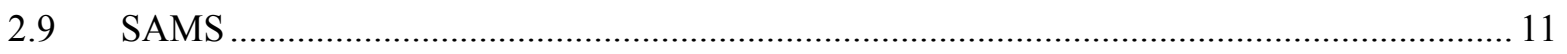

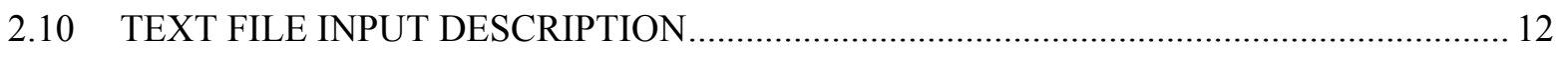

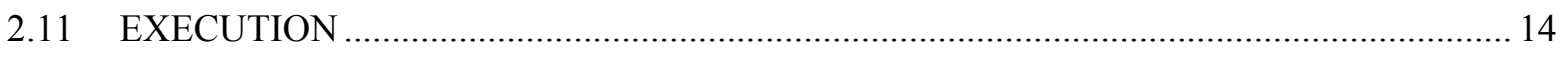

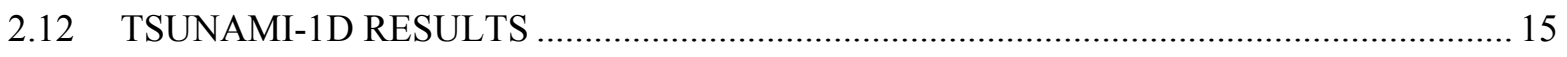

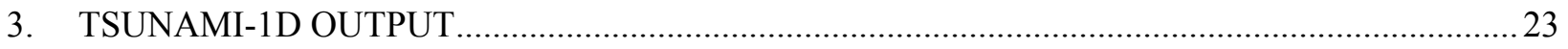

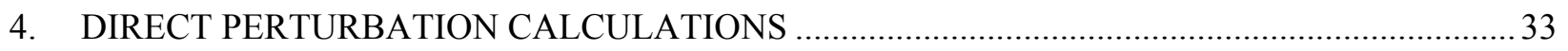

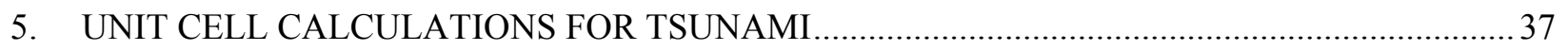

6. $\quad$ FISSION SPECTRUM (CHI) SENSITIVITY CALCULATIONS …............................................. 39

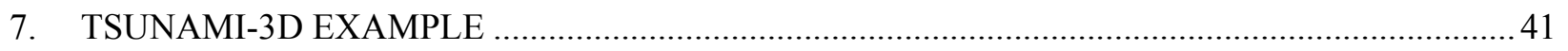

8. SELECTION OF BENCHMARK EXPERIMENTS ............................................................5

$8.1 \quad$ SENSITIVITY DATA DISTRIBUTED WITH ICSBEP HANDBOOK..............................5

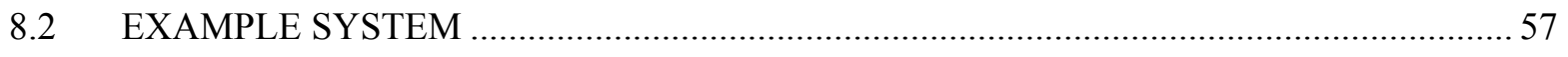

8.3 INTERPRETING SENSITIVITY DATA WITH VIBE ................................................. 58 


\section{CONTENTS (continued)}

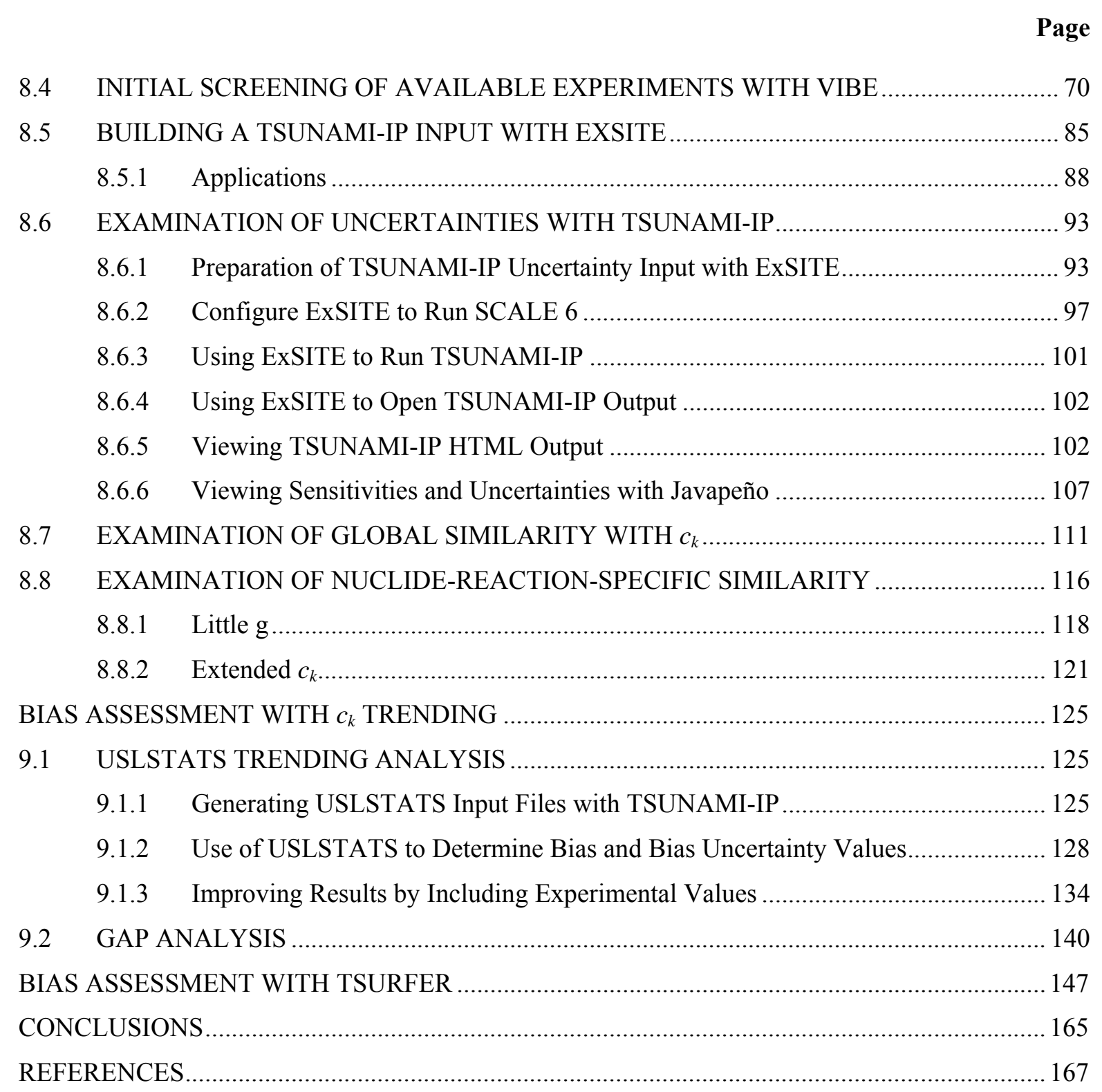




\section{LIST OF FIGURES}

Figure $\quad$ Page

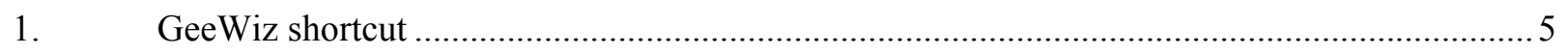

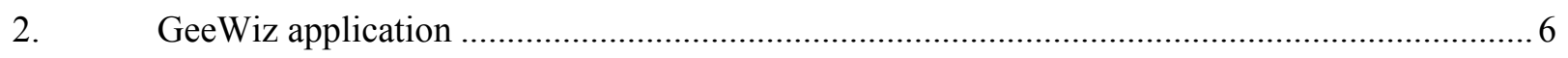

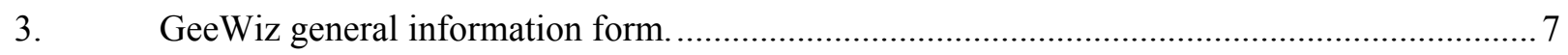

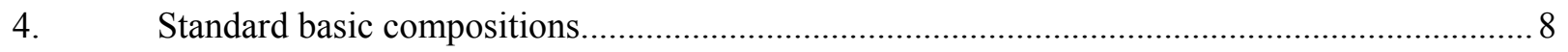

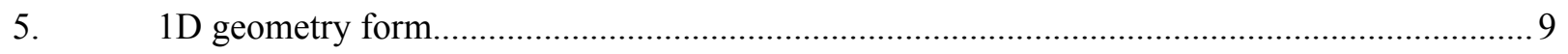

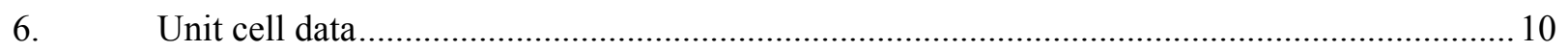

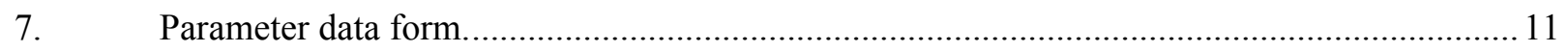

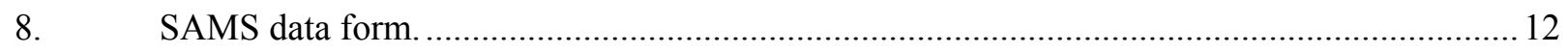

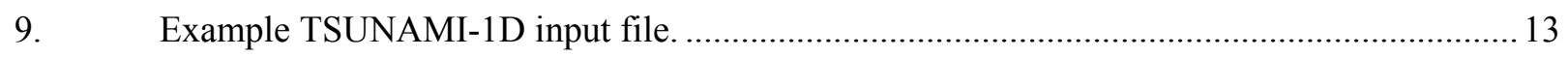

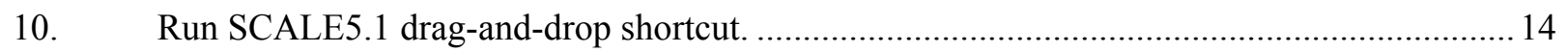

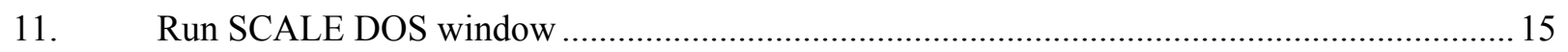

12. Files returned to user directory after TSUNAMI-1D calculation. ...................................... 15

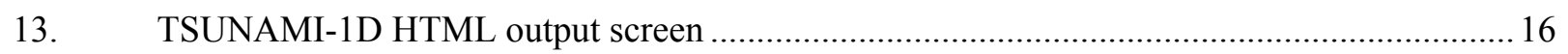

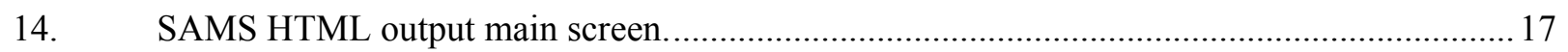

15. Energy, region, and mixture integrated sensitivity coefficients in TSUNAMI-1D

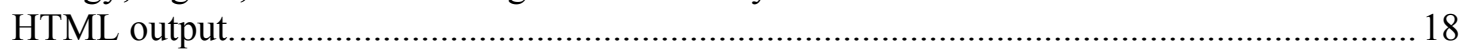

16. Total sensitivity coefficients by mixture in HTML output.................................................. 19

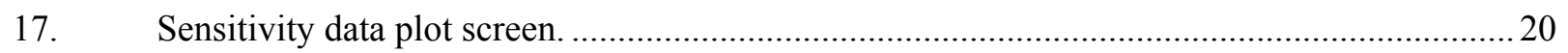

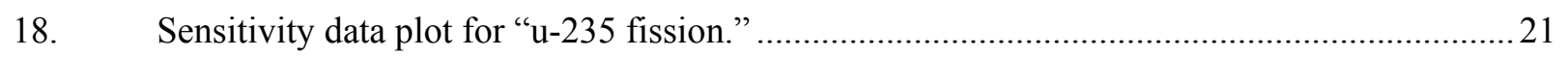

19. Uncertainty information for TSUNAMI-1D example problem........................................22

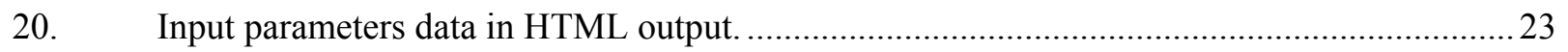

21. Summary of transport calculations in HTML output from TSUNAMI-1D..........................24

22. Energy, region, and mixture integrated sensitivity coefficients in TSUNAMI-1D

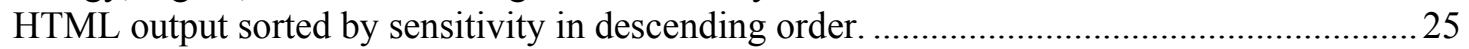

23. Energy and region integrated sensitivity coefficients in TSUNAMI-1D HTML

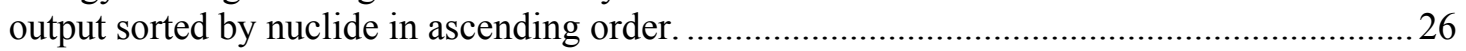

24. Sensitivity coefficients by material zone in TSUNAMI-1D HTML output.........................22

25. Sensitivity coefficients by nuclide in TSUNAMI-1D HTML output...................................28

26. Total sensitivity coefficients by mixture in TSUNAMI-1D HTML output. ........................29

27. Sensitivity data plot in TSUNAMI-1D HTML output with ${ }^{16} \mathrm{O}$ elastic scattering

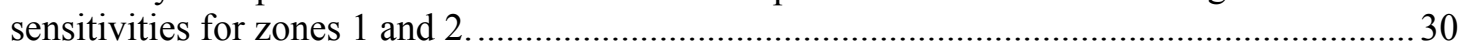

28. Problem characterization in TSUNAMI-1D HTML output. ............................................ 31

29. Uncertainty information in TSUNAMI-1D HTML output.................................................. 32 


\section{LIST OF FIGURES (continued)}

Figure

Page

30. Example of sensitivity profile indicating a unit cell modeling problem.................................38

31. Fission spectrum (chi) sensitivities for ${ }^{235} U$ from SCALE 5.1 and SCALE 6......................40

32. General information for TSUNAMI-3D example. .................................................. 41

33. Composition data for TSUNAMI-3D example. .......................................................... 42

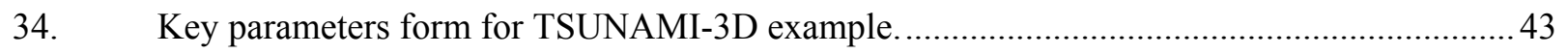

35. Numeric parameters for TSUNAMI-3D example. ................................................... 44

36. TSUNAMI-3D parameters for TSUNAMI-3D example.............................................. 45

37. Lattice cell data form for TSUNAMI-3D example. ...................................................... 46

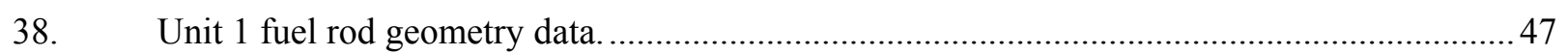

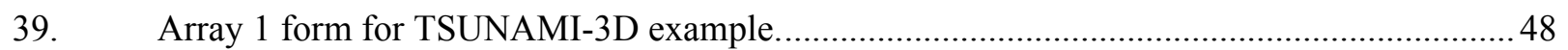

40. Global unit geometry data for TSUNAMI-3D example problem........................................4 49

41. SAMS data form for TSUNAMI-3D example problem. ..................................................50

42. Initial page of HTML output from TSUNAMI-3D example............................................. 51

43. KENO V.a forward calculation final results page for TSUNAMI-3D example.....................52

44. KENO V.a adjoint calculation final results page for TSUNAMI-3D example.....................52

45. Summary of transport calculation from TSUNAMI-3D example. ......................................53

46. Energy, region, and mixture integrated sensitivity coefficients from

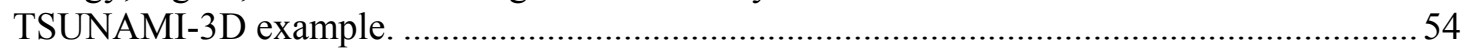

47. Sensitivity coefficients by region from TSUNAMI-3D example.......................................5

48. Sensitivity data plot from TSUNAMI-3D example........................................................55

49. Uncertainty information from TSUNAMI-3D example..................................................5

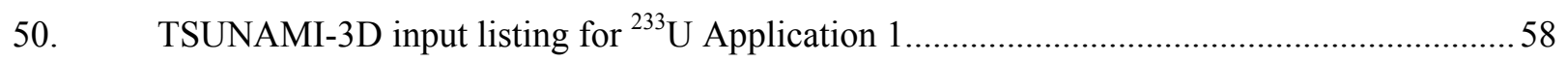

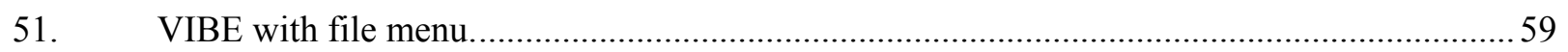

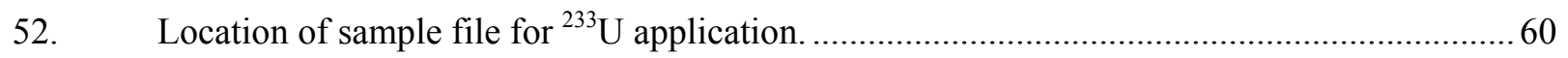

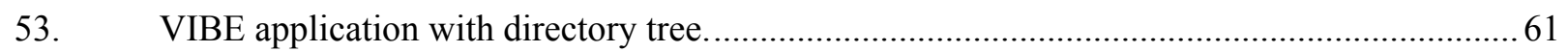

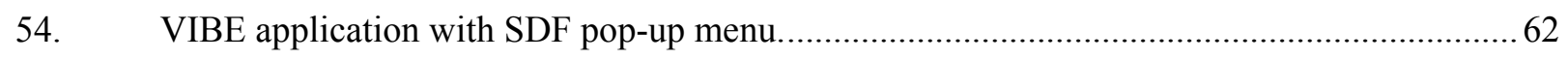

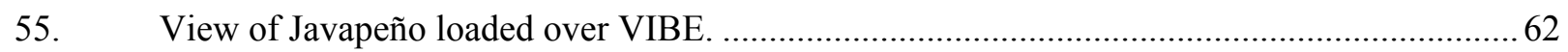

56. $\quad$ Plotting ${ }^{233} \mathrm{U}$ fission and n,gamma for ${ }^{233} \mathrm{U}$ application...................................................63

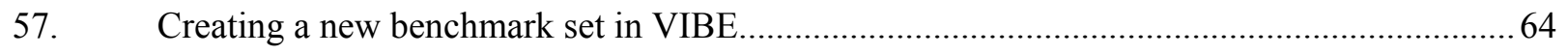

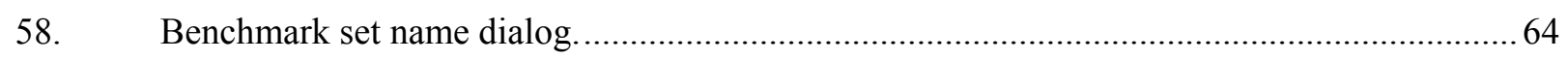

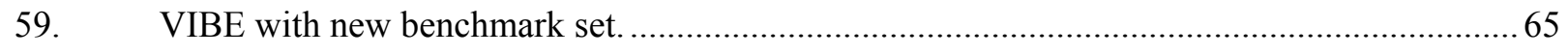

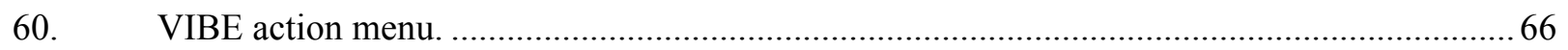




\section{LIST OF FIGURES (continued)}

Figure

Page

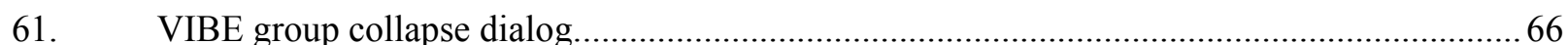

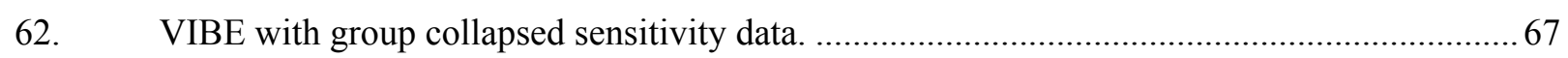

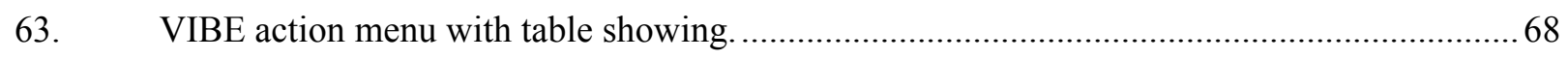

64. VIBE sort dialog for sorting by thermal sensitivities in descending order............................6 68

65. VIBE with thermal sensitivity data sorted in descending order. ........................................69

66. VIBE showing bottom of table with thermal sensitivity data sorted in descending order.......69

67. VIBE file chooser to select TSUNAMI data distributed in ICSBEP Handbook.................... 70

68. VIBE file tree pane with ICSBEP TSUNAMI sensitivity data selected. ............................. 71

69. Creation of new benchmark set for data distributed with ICSBEP Handbook.......................71

70. VIBE benchmark set dialog for ICSBEP Handbook data. ............................................... 72

71. VIBE with group collapsed sensitivity data for benchmarks in the ICSBEP Handbook ......... 72

72. $\quad$ VIBE filter dialog for ${ }^{233} \mathrm{U}$ nubar............................................................................ 73

73. VIBE with ${ }^{233}$ U filter applied to ICSBEP Handbook data.................................................. 74

74. $\quad$ VIBE with ${ }^{233} \mathrm{U}$ filler and descending sorting for thermal sensitivities applied to

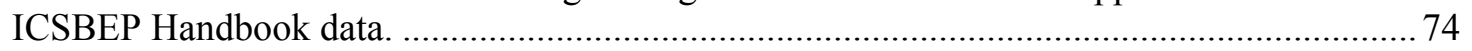

75. Values near 0.69 from VIBE with ${ }^{233} \mathrm{U}$ filter and descending sorting for thermal sensitivities applied to ICSBEP Handbook data......................................................... 75

76. $\quad$ VIBE Filter dialog for thermal ${ }^{233} \mathrm{U}$ nubar sensitivities with values $\geq 0.67$ and $\leq 0.71 \ldots \ldots \ldots . .75$

77. VIBE with ${ }^{233} \mathrm{U}$ nubar thermal sensitivities near 0.69 filter applied to ICSBEP

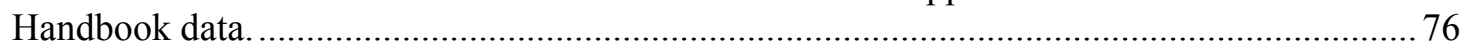

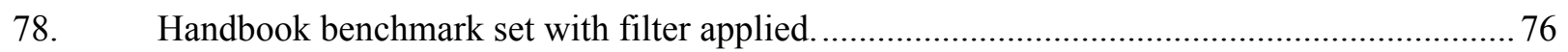

79. Javapeño reaction list with data for ${ }^{233} \mathrm{U}$ application and U233-SOL-THERM-016-003 ........ 77

80. Javapeño reaction list for plotting ${ }^{233} \mathrm{U}$ nubar sensitivity data for ${ }^{233} \mathrm{U}$ application in a

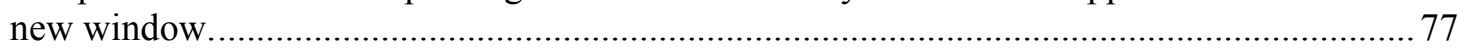

81. Javapeño reaction list for plotting ${ }^{233} \mathrm{U}$ nubar sensitivity data for

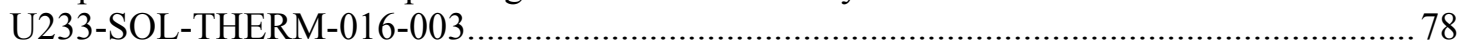

82. Javapeño plot of ${ }^{233} \mathrm{U}$ nubar sensitivities for ${ }^{233} \mathrm{U}$ application and

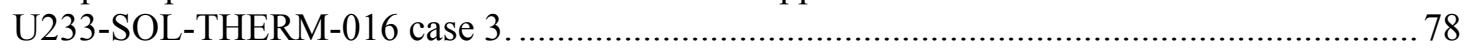

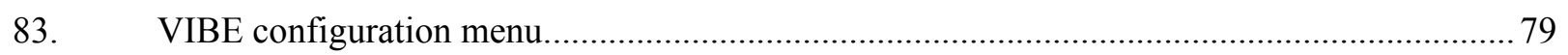

84. VIBE configuration manager dialog........................................................................... 79

85. File chooser to set location of DICE database............................................................. 80

86. Handbook benchmark set filtered for "ust016t." .......................................................... 80

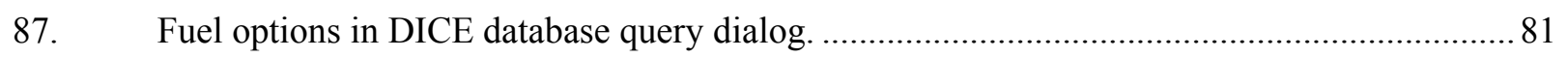

$88 . \quad$ Benchmark $k_{e f f}$ and uncertainty options in DICE database query dialog. ........................... 81 


\section{LIST OF FIGURES (continued)}

Figure $\quad$ Page

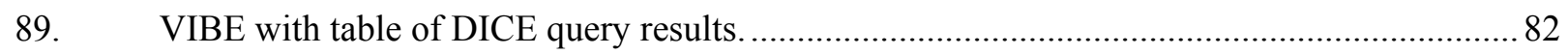

90. VIBE DICE query results for all U233-SOL-THERM experiments filtered from

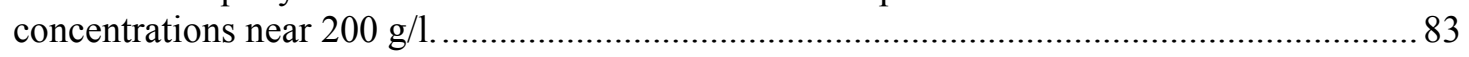

91. VIBE DICE query results for U233-SOL-THERM-002 benchmarks................................ 83

92. Sensitivity profiles for ${ }^{233} \mathrm{U}$ nubar from ${ }^{233} \mathrm{U}$ application and U233-SOL-THERM-002

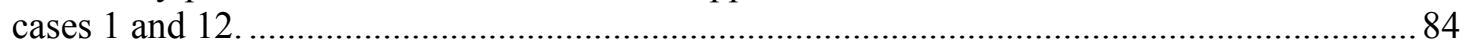

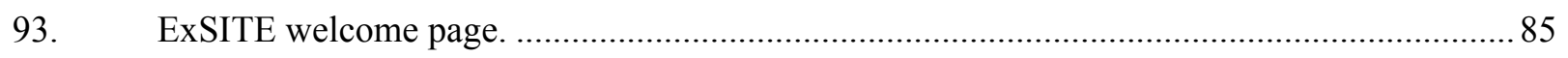

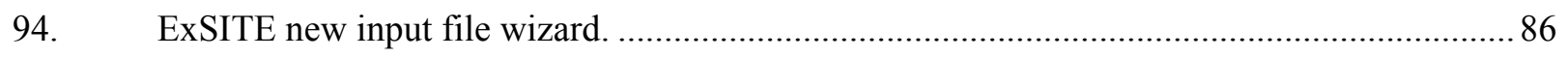

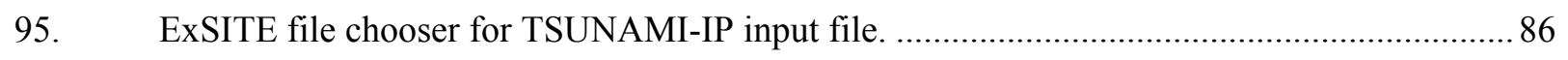

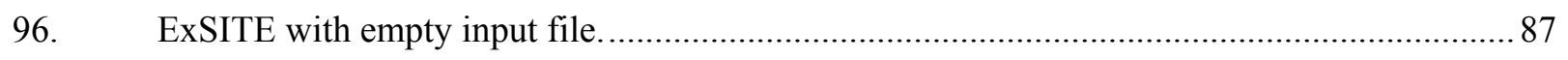

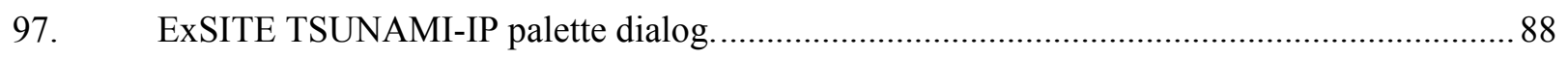

98. $\quad$ ExSITE applications tab for TSUNAMI-IP input with ${ }^{233}$ U application..............................89

99. $\quad$ ExSITE with ${ }^{233} \mathrm{U}$ application identified in TSUNAMI-IP input. ........................................ 89

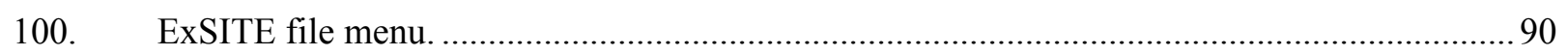

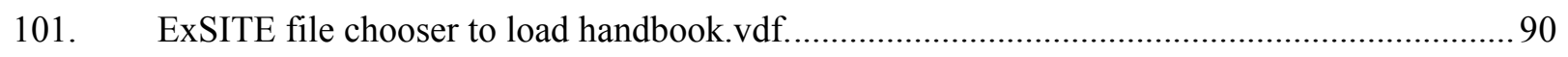

102. ExSITE with handbook data added to the palette and to the read experiments

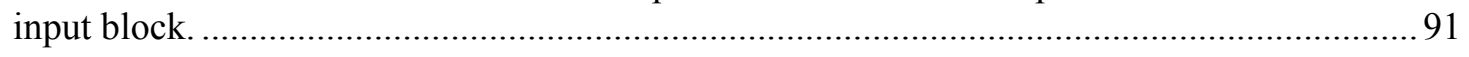

103. ExSITE TSUNAMI-IP dialog with handbook experiments loaded. ...................................92

104. ExSITE palette with pop-up menu. ............................................................................. 93

105. ExSITE TSUNAMI-IP input dialog for uncertainty data.............................................. 94

106. ExSITE TSUNAMI-IP input dialog for covariance data settings. .................................... 95

107. ExSITE TSUNAMI-IP input dialog for output edit settings. ..............................................96

108. ExSITE with TSUNAMI-IP input to compute uncertainties............................................ 97

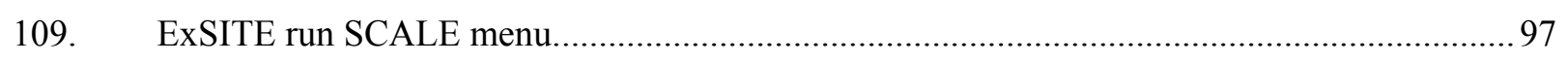

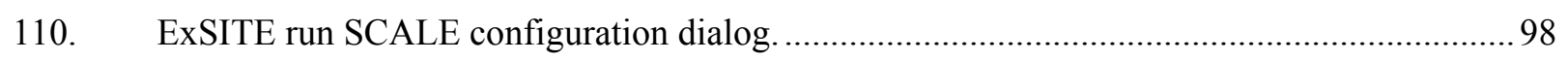

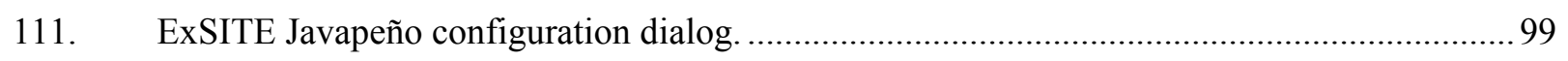

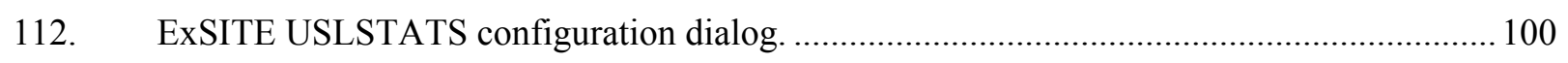

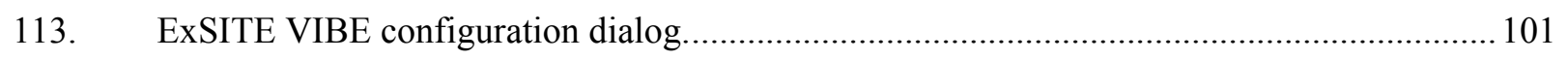

114. ExSITE process list window with TSUNAMI-IP input running. ...................................... 101

115. ExSITE process list window with TSUNAMI-IP input completed................................... 102

116. ExSITE toolbar icons to open output files................................................................... 102

117. SCALE initial screen for TSUNAMI-IP HTML output.................................................. 103

118. TSUNAMI-IP program verification page in HTML output. ............................................ 103 


\section{LIST OF FIGURES (continued)}

Figure $\quad$ Page

119. TSUNAMI-IP warning messages in HTML output. ................................................... 104

120. TSUNAMI-IP input parameters in HTML output....................................................... 104

121. TSUNAMI-IP applications list in HTML output. .................................................... 105

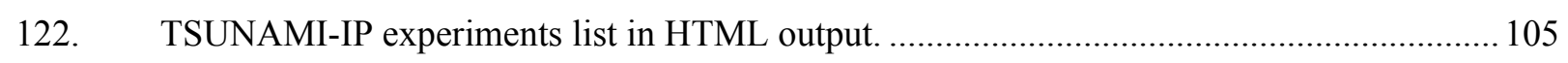

123. TSUNAMI-IP HTML format options in HTML output.................................................. 106

124. TSUNAMI-IP extended uncertainty edit in HTML output. ............................................. 107

125. Javapeño plot of sensitivities from ${ }^{233} \mathrm{U}$ application for top six contributors

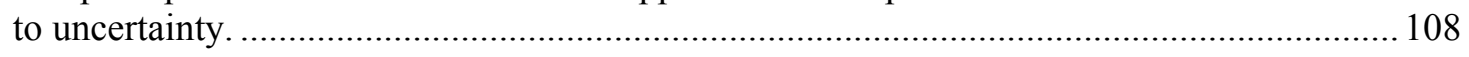

126. Javepeño file chooser to open "44groupcov" covariance file............................................. 109

127. Plotting standard deviation of cross-section-covariance matrix in a new window

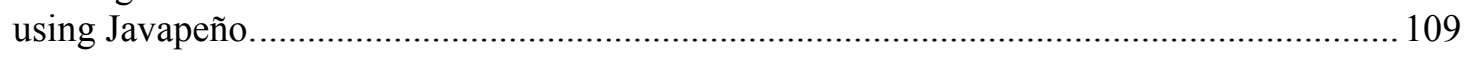

128. Plotting standard deviation of cross-section-covariance matrix using Javapeño................... 110

129. Javapeño plot of standard deviations of cross sections for ${ }^{233} \mathrm{U}$ application......................... 110

130. Energy-dependent uncertainty plot created with post-SCALE 6 Javapeño..........................111

131. Opening TSUNAMI-IP parameter palette in ExSITE................................................. 112

132. ExSITE palette dialog for TSUNAMI-IP parameter input........................................... 113

133. ExSITE with TSUNAMI-IP input updated to compute and print $c_{\mathrm{k}}$ values....................... 113

134. TSUNAMI-IP integral index values edit in HTML output. .......................................... 115

135. TSUNAMI-IP integral index values edit in HTML output sorted by descending $\mathrm{c}$ k.............115

136. TSUNAMI-IP $\mathrm{c}_{\mathrm{k}}$ summary edit in HTML output....................................................... 116

137. EXSITE TSUNAMI-IP parameter palette dialog with selections for

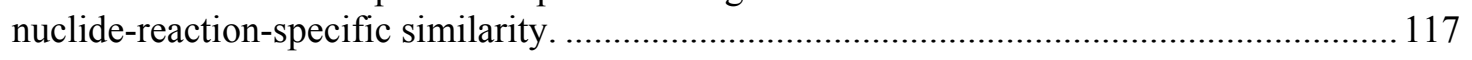

138. ExSITE with TSUNAMI-IP input updated to include nuclide-reaction-specific

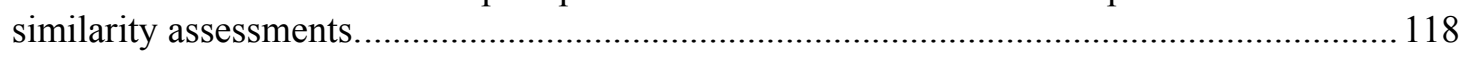

139. TSUNAMI-IP nuclide-reaction-specific integral index values for ${ }^{233}$ Upplication compared to itself in HTML output......................................................................... 119

140. TSUNAMI-IP nuclide-reaction-specific integral index values for ${ }^{233}$ application compared to U233-SOL-THERM-016 case 3 in HTML output......................................... 120

141. Javapeño plot of $\mathrm{H}$ scatter for ${ }^{233} \mathrm{U}$ application and U233-SOL-THERM-016 case 3 .......... 120

142. Javapeño plot of ${ }^{233} \mathrm{U}$ fission for ${ }^{233} \mathrm{U}$ application and U233-SOL-THERM-016 case 3........ 121

143. TSUNAMI-IP extended $c_{\mathrm{k}}$ edit for ${ }^{233} \mathrm{U}$ application compared to itself in HTML output...... 122

144. TSUNAMI-IP extended $c_{k}$ ed lit for ${ }^{233} \mathrm{U}$ application compared to U233-SOL-THERM-016 case 3 in HTML output.

145. ExSITE palette dialog for TSUNAMI-IP USLSTATS input parameters........................... 126 


\section{LIST OF FIGURES (continued)}

Figure $\quad$ Page

146. ExSITE auto-completion for TSUNAMI-IP parameter data ......................................... 127

147. Location of directory containing USLSTATS input files generated by TSUNAMI-IP. ........ 127

148. USLSTATS input files generated by TSUNAMI-IP................................................. 128

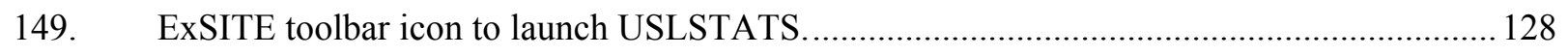

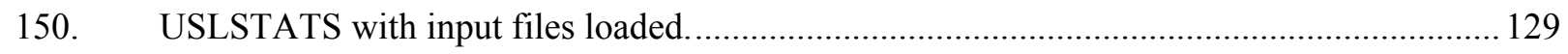

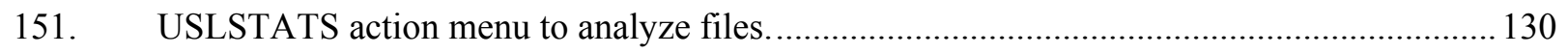

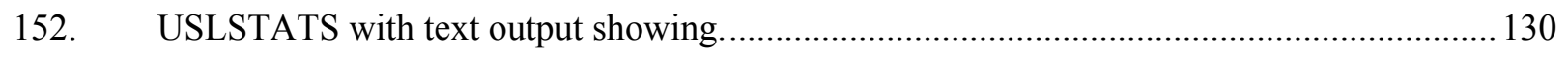

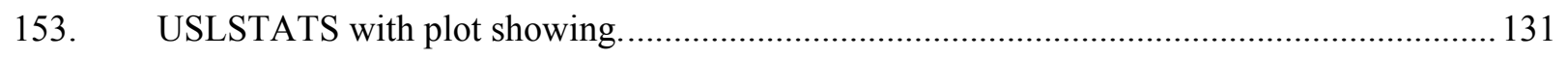

154. USLSTATS with Javapeño applet window menu. .................................................. 131

155. USLSTATS trend plot for experiments with $c{ }_{k} \geq 0.9$ for ${ }^{233} \mathrm{U}$ application.......................... 132

156. USLSTATS with Javapeño pop-up menu. ................................................................... 133

157. USLSTATS with Javapeño table of results. .................................................................... 133

158. ExSITE toolbar icons to launch VIBE..................................................................... 134

159. VIBE file menu item to open an existing benchmark set. ............................................. 134

160. VIBE file chooser to open the handbook benchmark set.............................................. 135

161. VIBE DICE database query for benchmark $k_{\text {eff }}$ and $k_{\text {eff }}$ uncertainty................................. 135

162. VIBE query results for benchmark $k_{\text {eff }}$ and $k_{\text {eff }}$ uncertainty........................................... 136

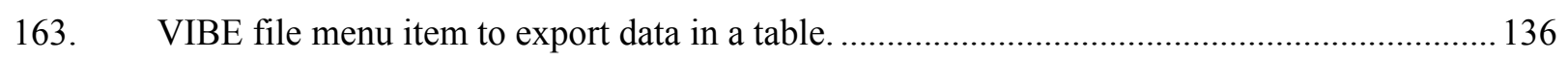

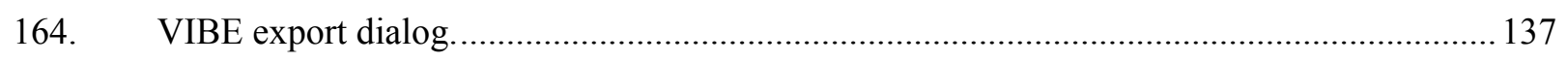

165. VIBE file chooser to export table data.................................................................... 137

166. ExSITE with TSUNAMI-IP input including benchmark $k_{\text {eff }}$ and benchmark $k_{\text {eff }}$

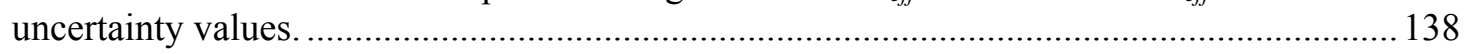

167. ULSTATS input using benchmark $k_{e f f}$ and benchmark $k_{e f f}$ uncertainty values.................... 139

168. USLSTATS trend plot including benchmark $k_{\text {eff }}$ and benchmark $k_{\text {eff }}$ uncertainties for experiments with $c_{k} \geq 0.9$ for ${ }^{233} U$ application................................................... 140

169. ExSITE with TSUNAMI-IP input updated to perform penalty calculations......................... 141

170. TSUNAMI-IP penalty summary edit in HTML output. ................................................ 142

171. TSUNAMI-IP extended penalty assessment edit in HTML output.................................. 142

172. ExSITE palette dialog for TSUNAMI-IP output edit parameters. ..................................... 143

173. ExSITE palette dialog to add reactions block to TSUNAMI-IP input. ............................... 144

174. ExSITE with TSUNAMI-IP input updated to add reactions data..................................... 145

175. TSUNAMI-IP composite sensitivity data plot in HTML output..................................... 146 


\section{LIST OF FIGURES (continued)}

Figure $\quad$ Page

176. Conversion of TSUNAMI-IP input to TSURFER input using ExSITE.............................. 148

177. ExSITE palette dialog for TSURFER calculation options parameters................................. 149

178. ExSITE palette dialog for TSURFER output edits parameters. ...................................... 150

179. ExSITE palette dialog for TSURFER covariance data parameters. .................................... 151

180. TSURFER input with updated parameter data in ExSITE. .......................................... 152

181. TSURFER correlation data in ExSITE ...................................................................... 153

182. TSURFER program verification page in HTML output................................................ 154

183. TSURFER warning message in HTML output............................................................. 154

184. TSURFER input response list in HTML output. .......................................................... 155

185. TSURFER application and bias summary in HTML output. .......................................... 156

186. TSURFER adjustment summary in HTML output......................................................... 157

187. TSURFER problem dimension edit in HTML output. ................................................. 158

188. TSURFER cross-section adjustments in HTML output. .................................................... 159

189. TSURFER cross-section adjustment plot in HTML output............................................. 160

190. Plot of energy-dependent bias and sensitivity for ${ }^{233} \mathrm{U}$ fission for ${ }^{233} \mathrm{U}$ application. ............. 161

191. ExSITE with TSUNAMI-IP input to compute postadjustment uncertainties........................ 162

192. TSUNAMI-IP extended uncertainty edit using postadjustment covariance data. ................. 163 



\begin{abstract}
This primer presents examples in the application of the SCALE/TSUNAMI tools to generate $k_{\text {eff }}$ sensitivity data for one- and three-dimensional models using TSUNAMI-1D and -3D and to examine uncertainties in the computed $k_{\text {eff }}$ values due to uncertainties in the cross-section data used in their calculation. The proper use of unit cell data and need for confirming the appropriate selection of input parameters through direct perturbations are described.

The uses of sensitivity and uncertainty data to identify and rank potential sources of computational bias in an application system and TSUNAMI tools for assessment of system similarity using sensitivity and uncertainty criteria are demonstrated. Uses of these criteria in trending analyses to assess computational biases, bias uncertainties, and gap analyses are also described. Additionally, an application of the data adjustment tool TSURFER is provided, including identification of specific details of sources of computational bias.
\end{abstract}





\section{INTRODUCTION}

This primer is intended to assist users with the mechanics of performing criticality safety validations

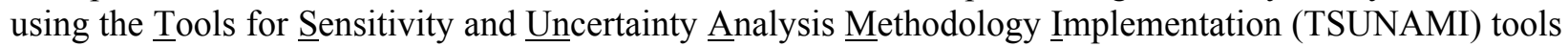
of SCALE 6. It is designed to demonstrate the most common functions of TSUNAMI in a step-by-step guide with numerous illustrations to clarify the mechanics of applying the tools. The example problems were selected to represent real systems yet be simple enough that the interested reader could quickly reproduce them while reading the primer. The information presented here is by no means an exhaustive review of all possible applications of TSUNAMI. It is hoped that future revisions to this document will provide additional examples of analyses that can be performed with TSUNAMI.

In this primer, many commonly used input data are described or demonstrated. However, this primer is not intended to substitute for the comprehensive documentation of the computational tools of SCALE

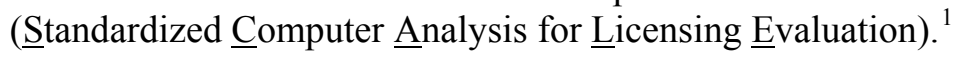

This primer evolved as SCALE 6 was under development to enable its release as part of the SCALE 6 distribution. Due to the parallel development of this document and the software it describes, some components of the software, especially the graphical user interfaces, will have changed by the time SCALE 6 is released. However, the authors believe that a change in some components of the analysis process does not diminish the value of this primer in enabling users to expand the application of the TSUNAMI methodology in their daily work.

\subsection{VALIDATION OF CODES AND DATA}

Modern neutron transport codes, such as the KENO codes in the SCALE code system and MCNP (Monte Carlo $\underline{N}$ Particle Transport Code) ${ }^{2}$ can predict $k_{\text {eff }}$ with a high degree of precision. Still, computational biases of a percent or more are often found when using these codes to model critical benchmark experiments. The primary source of this computational bias is believed to be errors in the cross-section data, as bounded by their uncertainties, which can be tabulated in cross-section-covariance data. To predict or bound the computational bias for a design system of interest, the American National Standards for Nuclear Criticality Safety in Operations with Fissionable Material Outside Reactors, ANSI/ANS-8.1-1998, ${ }^{3}$ and the American National Standard for Validation of Neutron Transport Methods for Nuclear Criticality Safety Calculations, ANSI/ANS-8.24-2007, calculations in the determination of subcritical limits for the design of fissionable material systems. The standards require validation of the analytical methods and data used in nuclear criticality safety calculations to quantify any computational bias and the uncertainty in the bias. The validation procedure must be conducted through comparison of the computed results with experimental data, and the design system for which the subcritical limit is established must fall within the area of applicability of the experiments chosen for validation. The 8.1 standard defines the area (or areas) of applicability as "the limiting ranges of material compositions, geometric arrangements, neutron-energy spectra, and other relevant parameters (e.g., heterogeneity, leakage, interaction, absorption, etc.) within which the bias of a computational method is established."

In compliance with the standards, the nuclear criticality safety community in the United States typically evaluates the computational biases and uncertainties of its computational methods and nuclear data through the use of trending analyses, usually a linear regression with a statistical confidence band. For a traditional trending analysis, a suite of experimental benchmarks is selected with physical characteristics that are similar to the corresponding values in the design system for which the subcritical limit is to be established. ${ }^{5}$ Some physical characteristics used to evaluate system similarity are fissile element(s), fissile concentration, moderator type, geometrical configuration, hydrogen-to-fissile atom ratios $(\mathrm{H} / \mathrm{X})$, 
average neutron-energy group causing fission, and energy of average neutron lethargy causing fission (EALF). Typically, the trending parameters are calculated as averages for each benchmark experiment.

Each of the experiments in the benchmark suite is modeled with the same code and neutron cross-section data that will be used in the criticality safety analysis of the design system. The difference between the measured and calculated values of $k_{\text {eff }}$, the effective neutron multiplication factor, of a critical experiment is considered to be the computational bias for that experiment. The expected computational bias of the design system is established through a trending analysis of the bias for all of the selected critical experiments as a function of their physical characteristics (e.g., H/X, EALF). The uncertainty in the bias is established through a statistical analysis of the trend, taking into account the uncertainty in each $k_{\text {eff }}$ data point and the distribution of the data.

\subsection{TSUNAMI TECHNIQUES FOR CODE VALIDATION}

The TSUNAMI software developed at Oak Ridge National Laboratory (ORNL) provides a unique means of determining the similarity of nuclear criticality experiments to safety applications. ${ }^{6}$ The basis of the TSUNAMI validation techniques is that computational biases are primarily caused by errors in the cross-section data, which are quantified in cross-section-covariance data. Instead of using one or more average physical parameters to characterize a system, TSUNAMI determines the uncertainty shared between two systems, which directly relates to the bias shared by the two systems. To accomplish this, the sensitivity of $k_{\text {eff }}$ to each groupwise nuclide-reaction specific cross section is computed for all systems considered in the analysis. Correlation coefficients are developed by propagating the uncertainties in neutron cross-section data to uncertainties in the computed neutron multiplication factor for experiments and safety applications through sensitivity coefficients. The bias in the experiments, as a function of correlated uncertainty with the intended application, is extrapolated to predict the bias and bias uncertainty in the target application.

\subsection{GAP ANALYSIS}

Even with advanced tools to identify benchmark experiments, criticality safety analysts occasionally find that the safety analysis models include some feature or material for which adequately similar, welldefined critical experiments do not exist to support validation. For example, the analyst may want to take credit for the presence of fission products in spent nuclear fuel or structural materials in shipping and storage configurations. In such cases, analysts sometimes rely on "expert judgment" to select an additional administrative margin to cover the validation weakness or to conclude that the impact on the calculated bias and bias uncertainty is negligible. TSUNAMI provides the means to estimate the potential impact on the application-specific bias and bias uncertainty resulting from nuclides not represented in the critical experiments.

\subsection{THE TOOLS OF TSUNAMI}

TSUNAMI was introduced in SCALE 5.0 and has been improved in each subsequent SCALE release 1 TSUNAMI through SCALE 5.1 has three major functionalities: one-dimensional (1D) sensitivity and uncertainty (S/U) analysis with TSUNAMI-1D, three-dimensional (3D) S/U analysis with TSUNAMI3D, and system-to-system similarity assessment through several S/U-based indices and parameters with TSUNAMI-IP. Additionally, sensitivity data and cross-section-covariance data can be visualized through the Javapeño plotting package, and biases and their uncertainty can be computed using the USLSTATS statistical package!

TSUNAMI-1D and TSUNAMI-3D compute energy-dependent sensitivity and uncertainty data for each nuclide in system model adjoint-based first-order linear perturbation theory and save the detailed 
sensitivity data to a file with the extension . sdf. Additionally, TSUNAMI-1D and -3D use the energy-dependent cross-section-covariance data to compute the uncertainty in each system's $k_{\text {eff }}$ value due to the cross-section-covariance data. TSUNAMI-IP uses the .sdf file generated from each system to compute correlation coefficients that determine the amount of shared uncertainty between each target application and each benchmark experiment considered in the analysis. TSUNAMI-IP offers a wide range of options for more detailed assessment of system-to-system similarity. Additionally, TSUNAMIIP can generate input for the USLSTATS trending analysis and compute a penalty, or additional margin, needed for the gap analysis.

\subsection{APPLICATIONS OF TSUNAMI}

TSUNAMI's primary function is to improve the ability to predict computational biases within the limitations of available experiments. TSUNAMI can be applied to a wide range of fissile systems and criticality experiments to help the analyst understand the sensitivity of $k_{\text {eff }}$ to different materials in the application system; understand which materials contribute to uncertainty in $k_{\text {eff, }}$ which is related to the computational bias; and identify experiments that have similar sensitivities and uncertainties and are expected to have biases similar to the application.

\subsection{LIMITATIONS OF TSUNAMI}

The generation of sensitivity coefficients is more complicated than ordinary $k_{\text {eff }}$ calculations. As will be explained in subsequent sections, TSUNAMI-3D can require a computer with multiple gigabytes of memory, and each calculation can run for hours. The user must carefully model not only the materials and geometry of the system, but also must provide unit cell specifications for the resonance self-shielding calculations. Up to $40 \%$ of the sensitivity of $k_{\text {eff }}$ to the cross-section data can come from the resonance self-shielding calculation. Inconsistent resonance self-shielding and criticality models will result in incorrect results and are the most common user errors. Results should be confirmed with direct perturbation methods and by plotting the sensitivity coefficients to identify anomalies that could indicate incorrect results. 



\section{TSUNAMI-1D EXAMPLE}

\subsection{GETTING STARTED}

TSUNAMI-1D is a code sequence that calculates the sensitivity of $k_{\text {eff }}$ of a 1D system (e.g., heterogeneous slab, cylinder, or sphere) to each nuclide and reaction in the system. We will introduce a basic 1D example to demonstrate the features of TSUNAMI-1D and to lead the user though the creation of an input file, execution of TSUNAMI-1D, and interpretation of the output. This example demonstrates techniques available in SCALE 5.1, but the same techniques are applicable to SCALE 6.

\subsection{QUICK START EXAMPLE}

A simple example selected to illustrate the TSUNAMI-1D sequence in SCALE is a $10 \%$ enriched $\mathrm{UO}_{2}$ sphere infinitely reflected by water. The input file generation will be discussed using both the SCALE Graphically Enhanced Editing Wizard (GeeWiz) user interface and a basic text file editor. The complete example input can be seen at the end of this chapter.

To begin, open GeeWiz by double-clicking the shortcut icon in the scale5.1 directory on your Windows desktop, shown in Fig. 1, or by selecting GeeWiz from the scale5.1 menu of the Windows Start menu. A GeeWiz application window, like that shown in Fig. 2, should appear. To begin the TSUNAMI-1D input, click the General button on the left toolbar. This button opens the general information form, shown in Fig. 3. Enter a title for the input file we will be creating, Example 1D. Next, choose the application that will be run; for our example, choose TSUNAMI1D - tsunami-1d, tsunami-1dc from the drop-down list. For the sequence, choose tsunami-1d from the list.

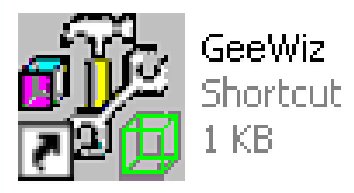

Fig. 1. GeeWiz shortcut. 


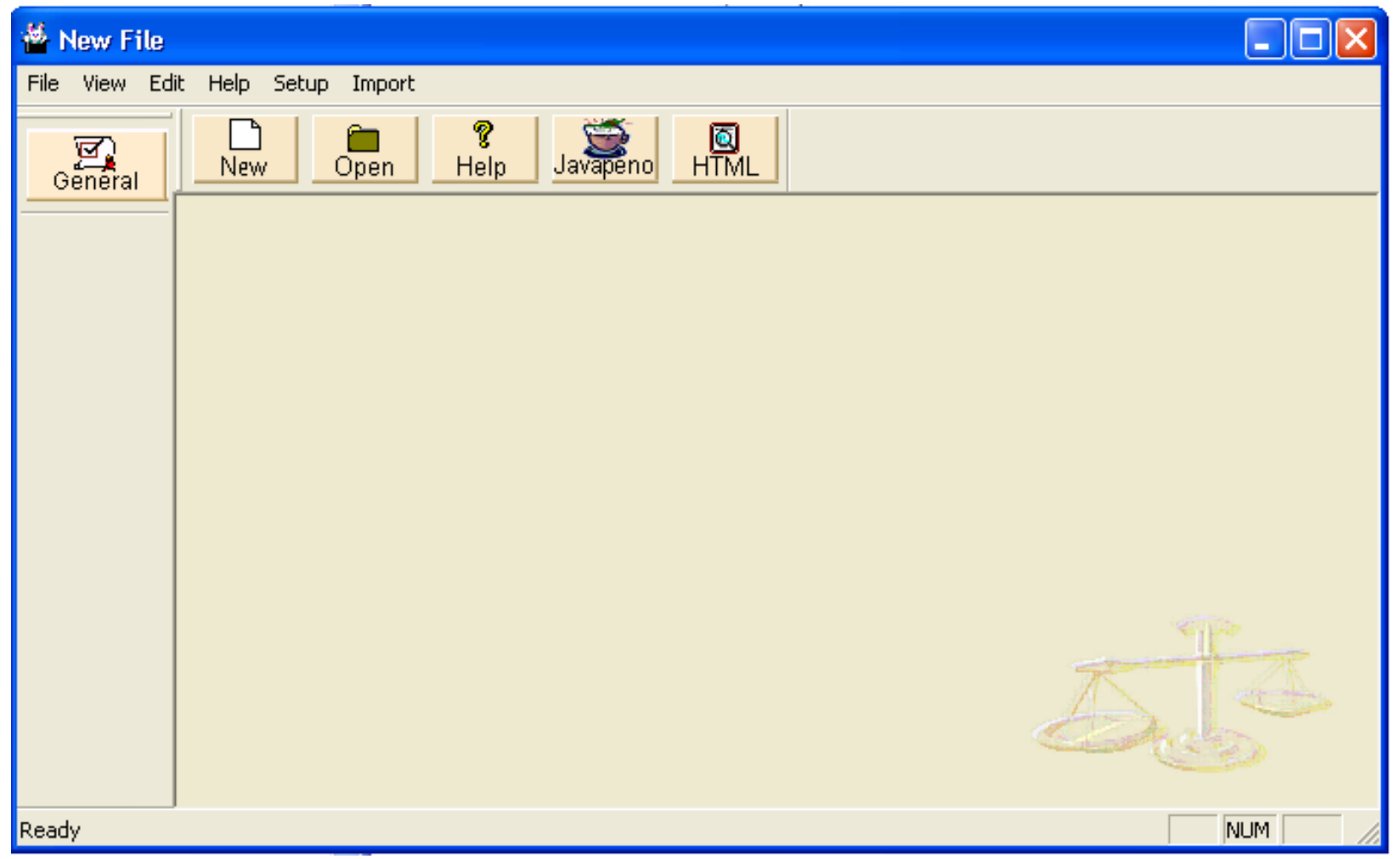

Fig. 2. GeeWiz application.

\subsection{CROSS-SECTION PROCESSOR}

Next, we need to choose a cross-section processor. We will use NITAWLST, the sensitivity version of the NITAWL cross-section processor. It offers reasonable results with a quicker run time. CENTRMST offers a more accurate solution, but requires significantly more run time and memory than NITAWLST. Note that for SCALE 5.1, CENTRMST must be used with ENDF/B-VI or later cross-section data; NITAWLST can process only ENDF/B-V or earlier resonance data. In SCALE 6, the CENTRMST module is not used as full-range Bondarenko factors are available for processing with BONAMIST, yielding excellent sensitivity analysis results with significantly reduced computer-system resource requirements. See the SCALE 6 Manual Sects. C.8.3.1 and C.9.3.1 for further guidance on use of cross-section processing modules in TSUNAMI-1D and TSUNAMI-3D calculations, respectively.

\subsection{CROSS-SECTION LIBRARY}

The cross-section library is the next option we need to select. In our example, we will use the 238-group ENDF/B-V 238groupndf5 cross-section library to minimize the run time for our example. The V6-238 cross-section library contains more recent data and is recommended for more accurate results. As mentioned previously, it requires the use of CENTRMST, which uses more central processing unit (CPU) time and memory. 


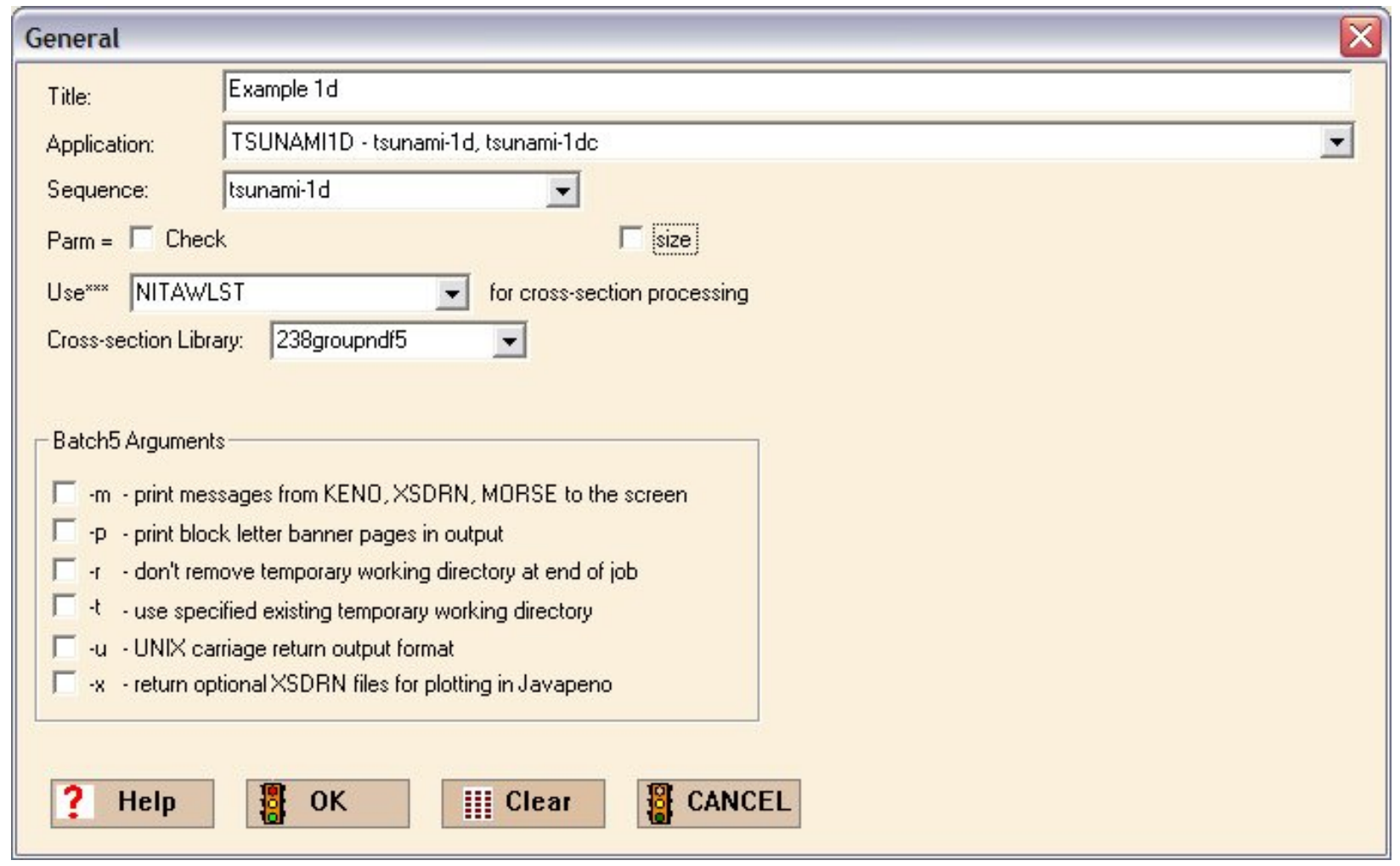

Fig. 3. GeeWiz general information form.

At the bottom of the General window is a box that has Batch5 Arguments. For this example, we will not select any of these. Finally, click the OK button at the bottom of the screen to complete this section.

\subsection{COMPOSITIONS}

To input the materials used in our example, click the Compositions button on the left toolbar. When the Compositions window opens, click the Create button on the top toolbar. On the next menu, click Basic Compositions, because our case has only $\mathrm{UO}_{2}$ and water. We will designate mixture 1 as $\mathrm{UO}_{2}$ and mixture 2 as water. When entering the data for $\mathrm{UO}_{2}$, change the isotopic distribution to reflect the $10 \%$ enrichment by clicking the Edit Selection button under the isotopic distribution report. Next to ${ }^{234} \mathrm{U}$, or 92234, enter 0.0 ; next to ${ }^{235} \mathrm{U}$, or 92235, enter 10.0; next to ${ }^{238} \mathrm{U}$, or 92238, enter 90.0. We will accept the default temperature of $300 \mathrm{~K}$, which is roughly room temperature. We will also change the density multiplier to 0.95 to represent $95 \%$ theoretical density.

Now that the information for mixture 1 is complete, click the New button at the top of the window, and again click Basic Composition. GeeWiz automatically increments the mixture number to 2 by the Mixture label. Select h2o from the drop-down menu. We will assume the default density multiplier (1) and temperature $(300 \mathrm{~K})$ for the water mixture to obtain room-temperature water. Press the OK button at the top of the window. You can quickly review the entered composition data by viewing the Standard Basic Compositions table, shown in Fig. 4. If everything looks correct, close the window. 


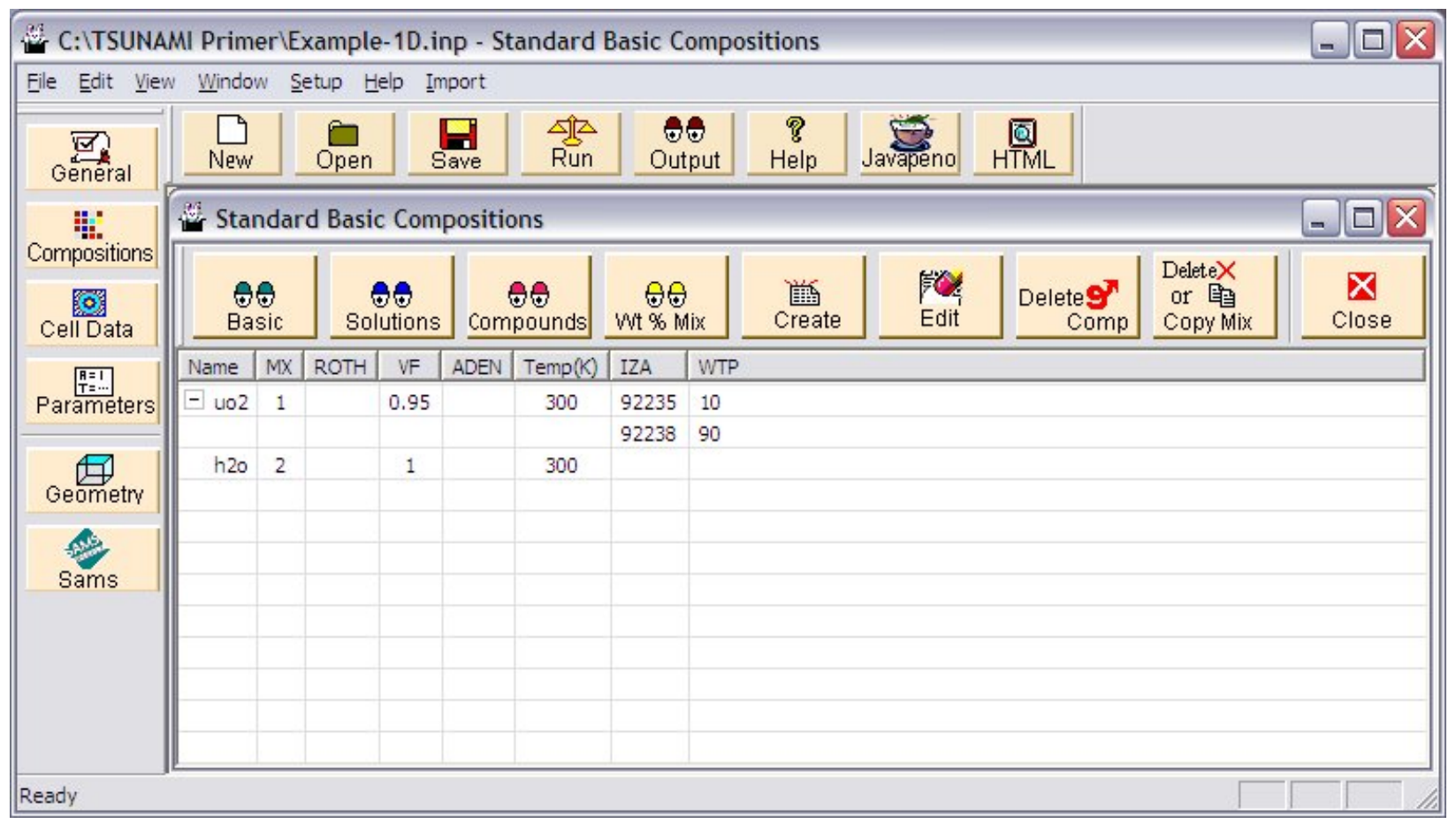

Fig. 4. Standard basic compositions.

\subsection{GEOMETRY}

With the compositions entered in our model, we see more options open to us on the left toolbar. Click on the Geometry button to open the 1D geometry form, as shown in Fig. 5. In the Geometry Type menu, select spherical. We will leave the boundary conditions as they are (left $=$ reflected, right $=$ vacuum). Click the Insert button above the table, and this will open a new window. The radius of the $\mathrm{UO}_{2}$ sphere is $57.5 \mathrm{~cm}$. Enter that value, select mixture 1 from the menu, and press $0 \mathrm{~K}$. Click the Insert button again and repeat the process for water, which will have a radius of $87.5 \mathrm{~cm}$. Check the inputs in the table, and click the OK button below the table. 


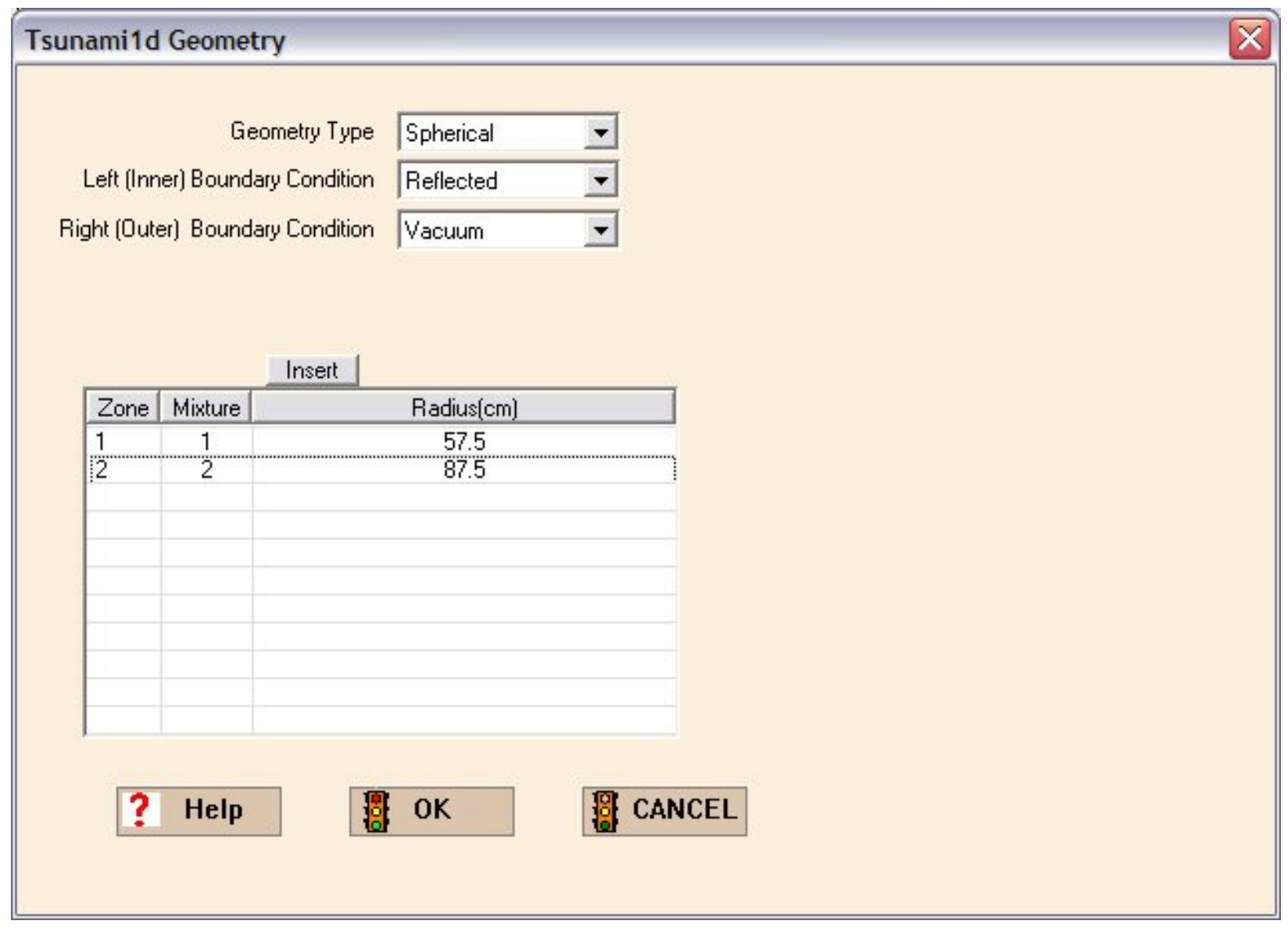

Fig. 5. $1 D$ geometry form

\subsection{CELL DATA}

Next, we will specify the unit cell data, which provides models for the resonance self-shielding models. Click on the Cell Data button on the left toolbar. Creating a 1D model usually requires the MultiRegion option. Click that tab at the top of the window, as shown in Fig. 6. A new cell can be created by clicking the new cell button on the top toolbar. This window is similar to the geometry window, and we will input the same dimensions and the same boundary conditions for our spherical case. Once the data are entered, click the Close button.

Briefly, we will discuss the other options that can be created in the Cell Data window. Lattice Cell should be used with repetitive lattice geometries, such as fuel rods in an assembly. The infinite homogenous medium option, labeled Infhommedium, should be used only if there is no interstitial moderating material. This option assumes that each mixture is infinitely large and its flux spectrum is not affected by other neighboring mixtures. Any mixture not placed in a Lattice Cell or MultiRegion unit cell will be treated as an infinite homogenous medium. If we used this option in our case, improper resonance self-shielding of the uranium cross sections would produce erroneous results.

The use of consistent cell data and geometry models is very important for TSUNAMI calculations, as will be explained in Sect. 5 . 


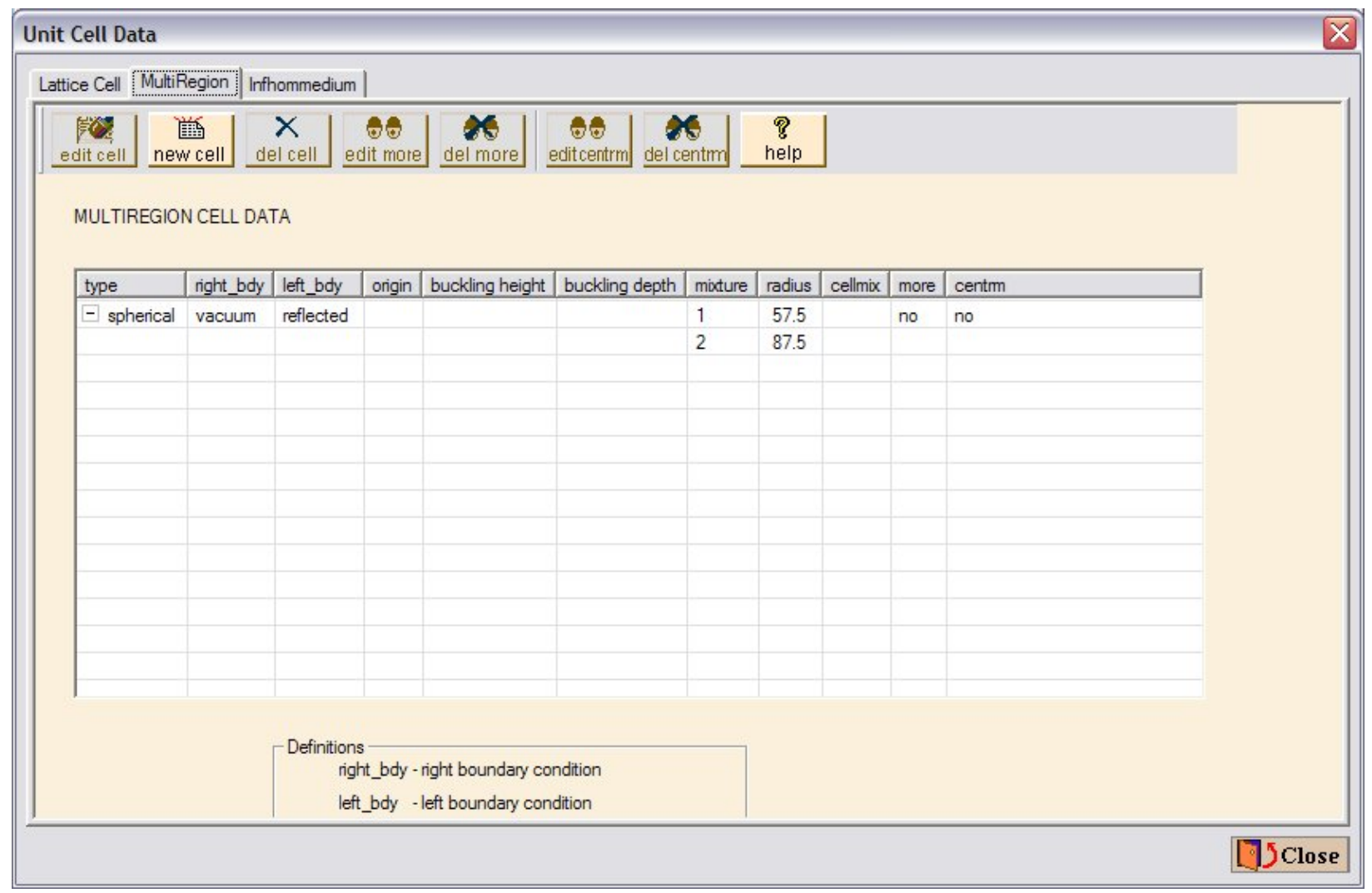

Fig. 6. Unit cell data

\subsection{PARAMETERS}

Parameter input allows the user to control the forward and adjoint criticality calculations performed with XSDRNPM. Choose the Parameters button on the left toolbar. The only parameter that we will change is the angular quadrature. Specify $I S N=32$, as shown in Fig. 7, to improve the accuracy of the calculation, then click OK to close the form. 


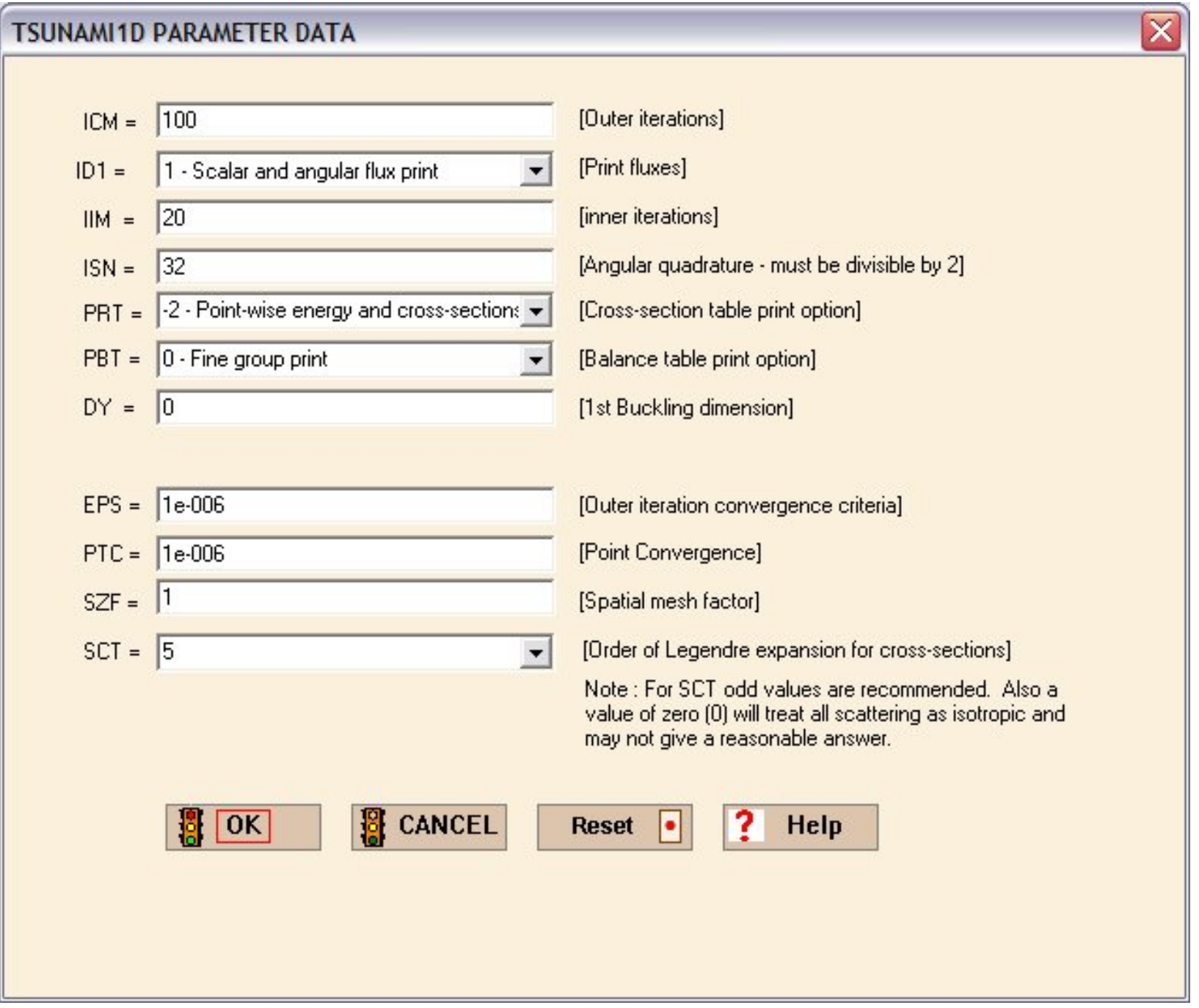

Fig. 7. Parameter data form.

\subsection{SAMS}

SAMS is the Sensitivity Analysis Module for SCALE that actually computes the sensitivity and uncertainty data using data generated in the forward and adjoint criticality calculations as well as the resonance self-shielding calculations. There are some user controls for SAMS. To access these controls, press the SAMS button on the left toolbar to open the SAMS data form, as shown in Fig. 8. We will use the makeimp option (already checked by default) to make implicit sensitivity coefficients, and the prtgeom and prtimp options to print the sensitivity data by geometry region and the implicit sensitivities, respectively. Click OK to accept the changes and close the window. 


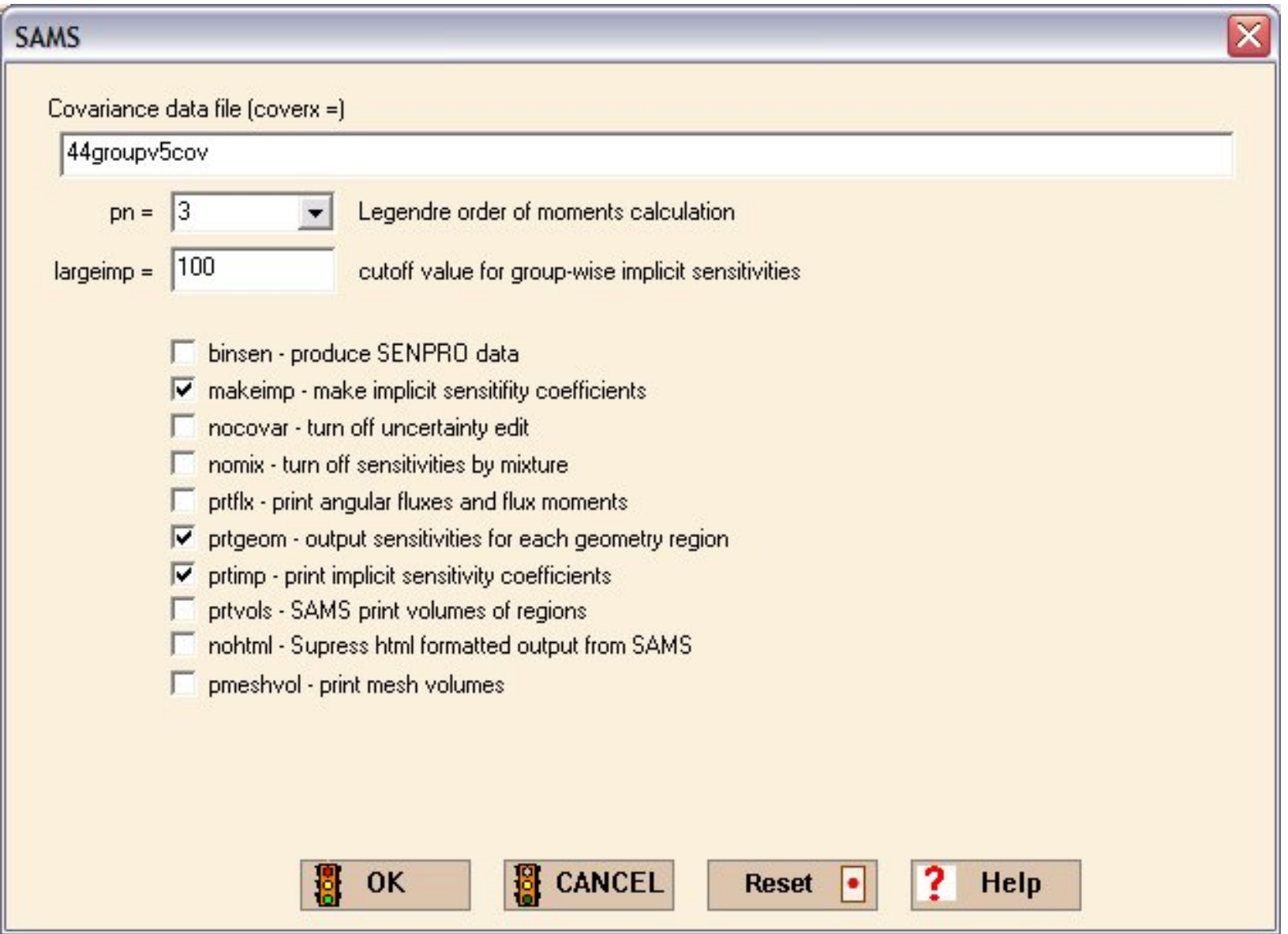

Fig. 8. SAMS data form.

At this point the input is complete, so click on the Run button on the top toolbar. You will be prompted to save the input file. (Enter Example-1D.inp as the file name.)

\subsection{TEXT FILE INPUT DESCRIPTION}

The input for TSUNAMI-1D is actually a plain text file that can be generated and edited using a basic text editor. A complete description of the TSUNAMI-1D input file format is provided in Sect. C8.3 of the SCALE Manual. The text file that is generated by GeeWiz from our example is shown below in Fig. 9 . Note that the line numbers in the left column are not part of the input file. They are included to facilitate the following description of the input file. 


\begin{tabular}{|c|c|}
\hline $\begin{array}{c}\text { Line } \\
\text { Number }\end{array}$ & Input File \\
\hline 1 & $=$ tsunami $-1 d$ parm $=($ nitawlst $)$ \\
\hline 2 & Example 1D \\
\hline 3 & $238 g$ roupndf5 \\
\hline 4 & read composition \\
\hline 5 & 10.95300 \\
\hline 6 & 9223510 \\
\hline 7 & $9223890 \quad$ end \\
\hline 8 & 21300 end \\
\hline 9 & end composition \\
\hline 10 & read celldata \\
\hline 11 & multiregion spherical left_bdy=reflected right_bdy=vacuum end \\
\hline 12 & $1 \quad 57.5$ \\
\hline 13 & 87.5 \\
\hline 14 & end zone \\
\hline 15 & end celldata \\
\hline 16 & read parameter \\
\hline 17 & isn $=32$ \\
\hline 18 & end parameter \\
\hline 19 & read geometry \\
\hline 20 & spherical vacuum reflected end \\
\hline 21 & 157.5 \\
\hline 22 & 287.5 \\
\hline 23 & end geometry \\
\hline 24 & read sams \\
\hline 25 & prtgeom \\
\hline 26 & prtimp \\
\hline 27 & end sams \\
\hline 28 & end \\
\hline
\end{tabular}

Fig. 9. Example TSUNAMI-1D input file.

The first line of the file specifies which SCALE sequence to run and any optional parameters. Here we are running the =tsunami-1d sequence using parm=(nitawlst), which specifies using NITAWLST for the resonance self-shielding module. It is important to note that you should use spaces, not tabs, to separate input parameters. Line 2 is the title for this input. In line 3 we indicate the cross-section library used. Refer to Table M4.1.1 in Sect. M4 of the SCALE Manual for a list of cross-section library names. Note that NITAWLST is only appropriate for use with libraries previous to ENDF/B-VI, such as the 238-group ENDF/B-V library used here. For ENDF/B-VI and later libraries, CENTRMST or BONAMIST must be used to correctly process the resonance parameter representation when using SCALE 5 or SCALE 6, respectively.

The composition data block begins on line 4. Each data block begins with a read... statement and terminates with a corresponding end... statement. The general format of each composition is as follows: composition name, mixture number, density multiplier, temperature, isotopic distribution in weight percent (if applicable). The SCALE basic standard composition names are documented in Sect. M8 of the SCALE Manual. 
The next block contains unit cell data and begins on line 10. As many unit cells as needed may be specified. In this case we specify multiregion as the unit cell type and spherical as the geometry type for the multiregion cell. These keywords are followed by the boundary conditions as seen in line 11. The multiregion geometry input for each region, or zone, is as follows: mixture number and dimension (in centimeters). The geometry input is terminated with the keywords end zone followed by the end celldata keywords to terminate the data block.

The parameter block begins on line 16. Only nondefault parameter values need to be specified. For this example, isn $=32$ is specified to change the angular quadrature to 32 .

The geometry block is next, beginning on line 19. In this block, the 1D geometry used for the forward and adjoint XSDRNPM calculations is specified. The input format is similar to the multiregion geometry input on lines 11-13. The format syntax for the geometry block is documented in Sect. C8.3.3.1 of the SCALE Manual.

The final data block is the sams block, beginning on line 24. This block includes only changes made to the default values. We included two more options: prtgeom to print the sensitivities by geometry region and prtimp to print the implicit sensitivities.

A SCALE input file is always terminated by an end statement beginning in column 1. Once saved, your input should be ready to execute in SCALE.

\subsection{EXECUTION}

Within GeeWiz, clicking the Run button on the top toolbar, shown in Fig. 2, will execute the problem in SCALE. SCALE can be run on a text input file by simply dropping the file on the Run SCALE5.1 shortcut, shown in Fig. 10, in the SCALE 5.1 directory that is added to your desktop by the SCALE installer. A similar directory and shortcut are provided with SCALE 6. When you execute SCALE with either method, a DOS window similar to the one in Fig. 11 will appear. On an Intel Core2 CPU (1.86 GHz and 1 GB RAM), the example problem took about 10 min to complete. When execution is complete, you may close the DOS window by clicking the $\mathrm{X}$ at the top right corner.

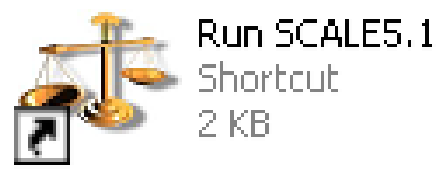

Fig. 10. Run SCALE5.1 drag-and-drop shortcut. 


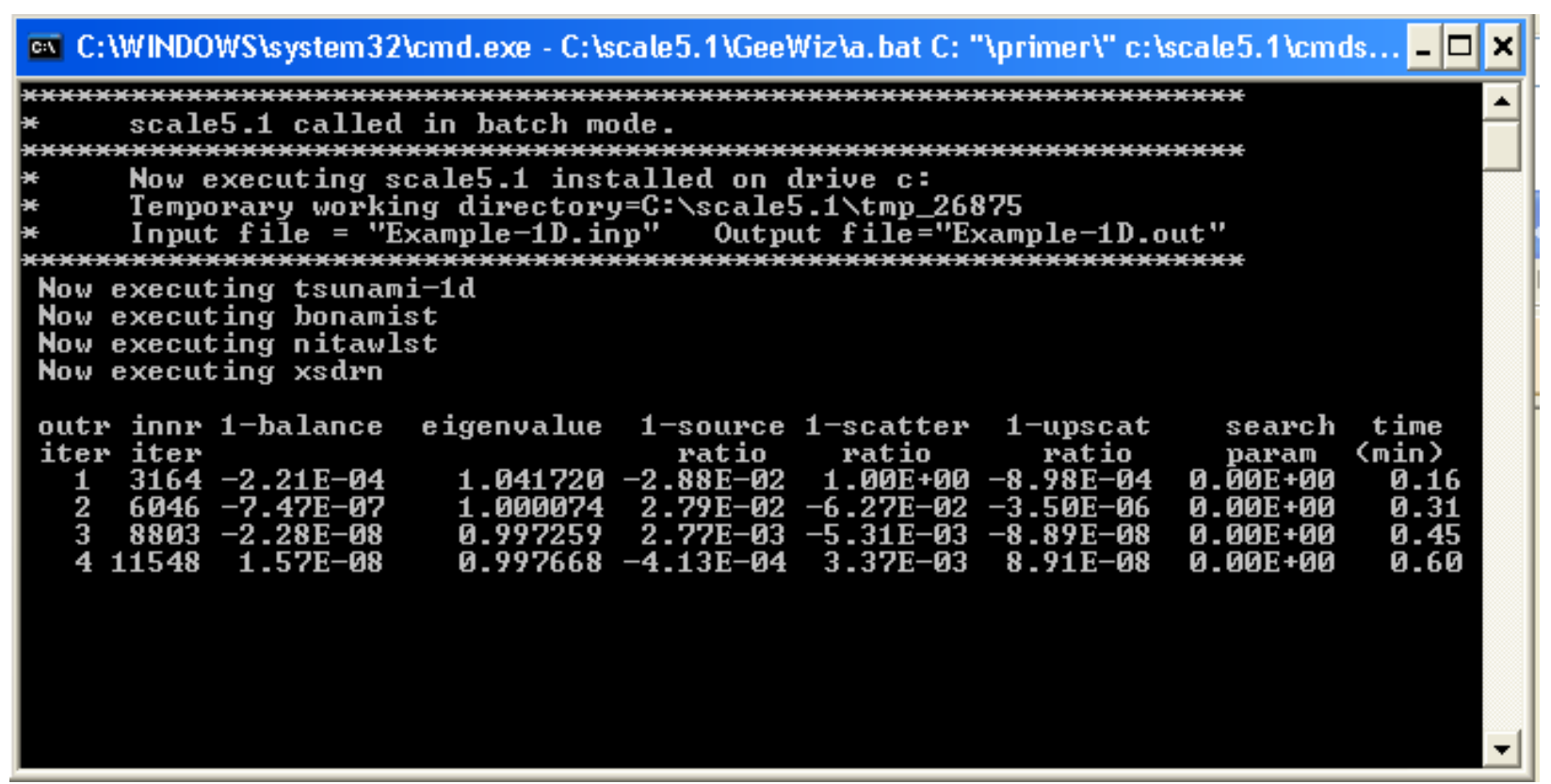

Fig. 11. Run SCALE DOS window.

\subsection{TSUNAMI-1D RESULTS}

Once execution is complete, SCALE returns the results of TSUNAMI-1D to the same directory where you saved your input file. In this case, plain text output is stored as Example-1D.out, HTML-formatted output is saved as Example-1D.html, with additional files in directories called Example-1D.htmd and applet_resources. Additionally, detailed energy-dependent sensitivity data are available for plotting and use in TSUNAMI-IP in the sensitivity data file (SDF) Example-1D. sdf. These files are shown in Fig. 12.

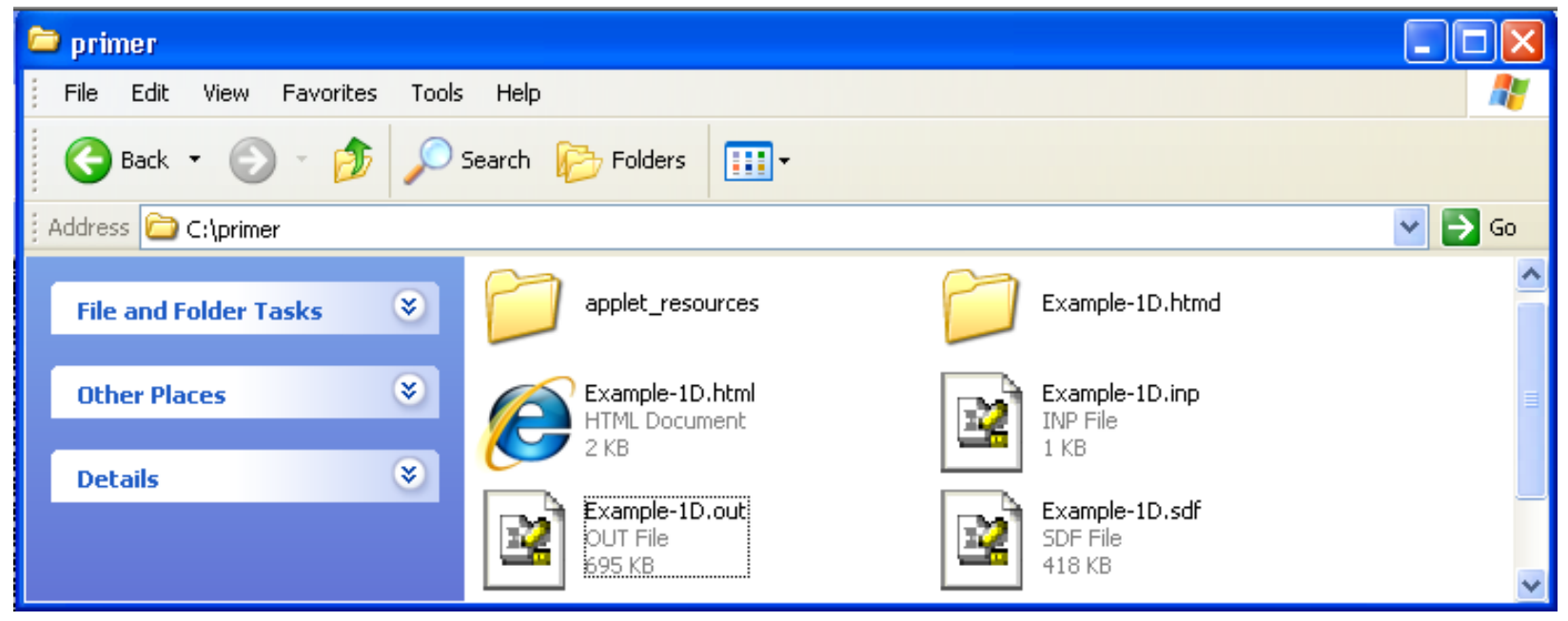

Fig. 12. Files returned to user directory after TSUNAMI-1D calculation. 
A detailed description of the entire output is presented in the next section, but the highlights of TSUNAMI-1D HTML output are presented here. Note that only the SAMS module output from TSUNAMI-1D is currently available in HTML format. The plain text output file contains the results from the resonance self-shielding calculations, the XSDRNPM transport calculations, and the SAMS sensitivity and uncertainty calculations. To view the output in HTML, launch the HTML browser by selecting the HTML icon on the top toolbar in GeeWiz. To view the SAMS HTML output, click on the 1. SAMS hyperlink on the list under the job title, example 1d, as shown in Fig. 13. Note that Java scripts must be enabled to run on your computer's browser to use some interactive features of the HTML output. Some browsers will present a warning message regarding ActiveX content. It is recommended that you select Allow Blocked Content to enable the full functionality of the HTML output. The blocked content can be permanently allowed by changing the security settings of your web browser. Please consult with your system administrator to ensure compliance with security protocols.

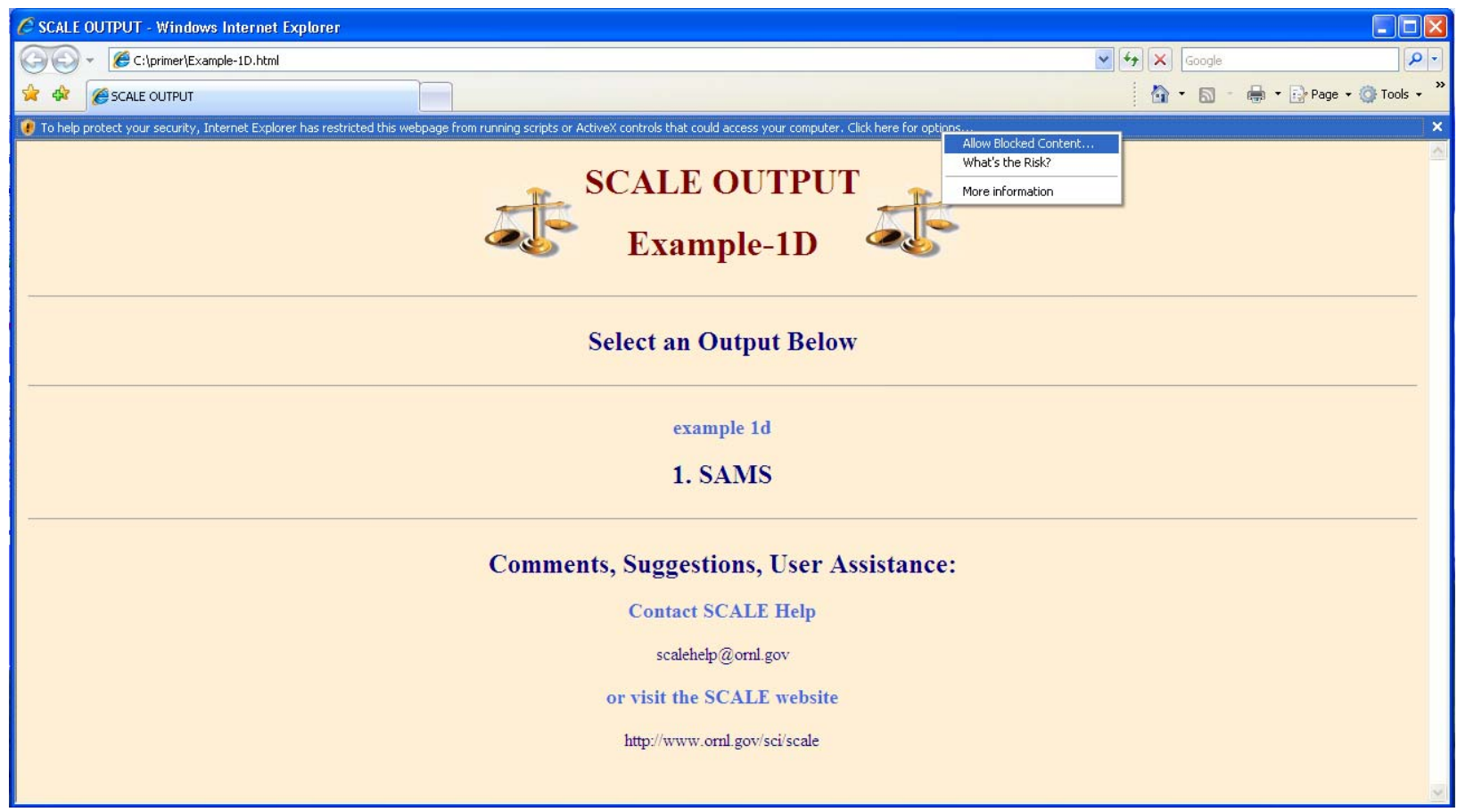

Fig. 13. TSUNAMI-1D HTML output screen. 
Program verification information is then displayed, and other information can be accessed through the menu on the left side of the screen, as shown Fig. 14. If any warning or error messages were generated during the SAMS calculation, they would be displayed in a Messages menu on the left side of the screen. It is always important to review any messages and assess their potential impact on your results.

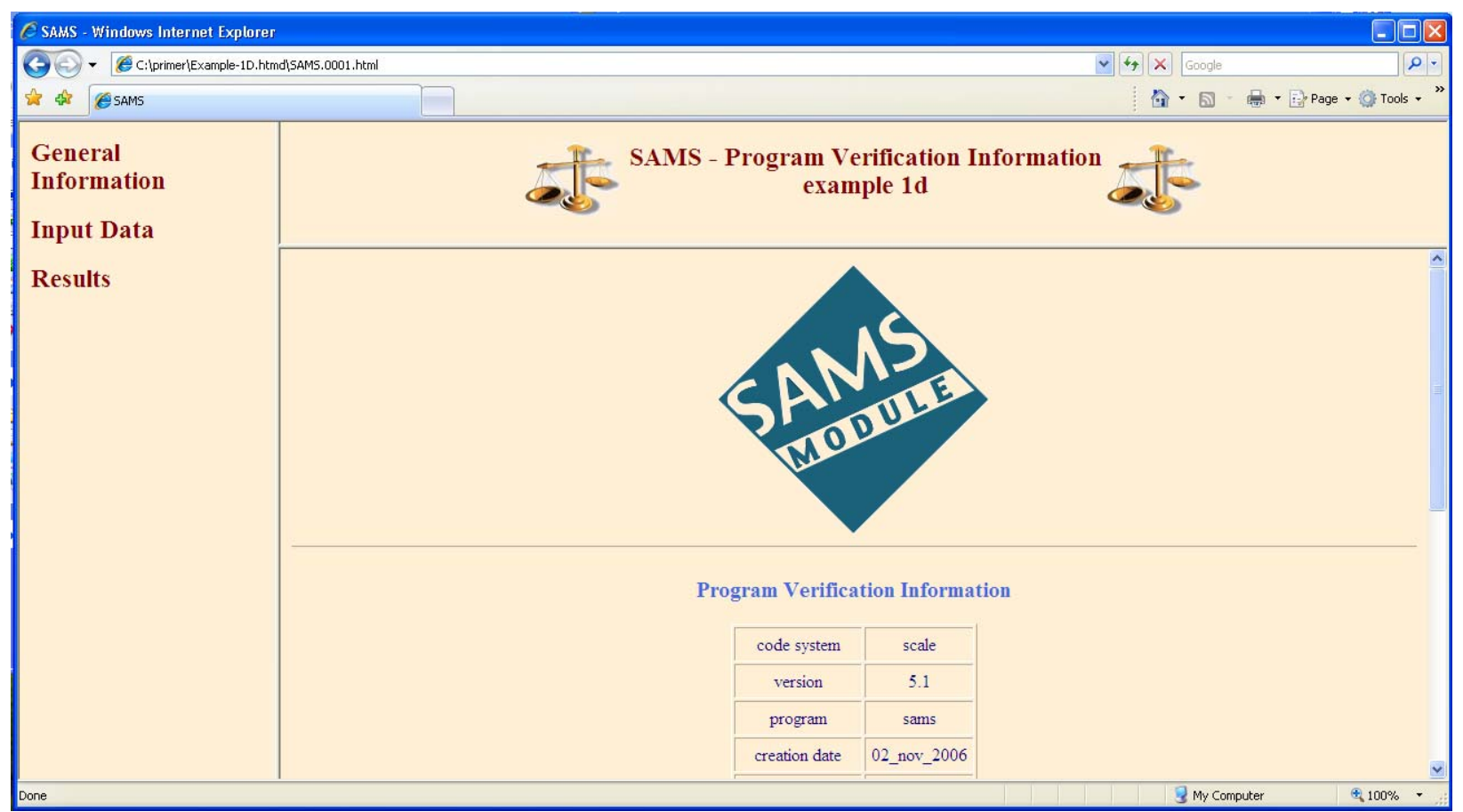

Fig. 14. SAMS HTML output main screen. 
Click on the Results menu item to open a new list of results in the menu on the left side of the screen. Click on the Energy, Region and Mixture Integrated Sensitivity Coefficients for this Problem option, which is the first listed option, to display the sensitivities listed by isotope, as shown in Fig. 15. The results shown in this table represent the percentage change in $k_{\text {eff }}$ one would observe by increasing the cross section for each nuclide reaction shown by $1 \%$ uniformly in all energy groups. From the data shown, a uniform $1 \%$ increase in the ${ }^{1} \mathrm{H}$ elastic scattering cross section would result in a $0.022292 \%$ increase in $k_{\text {eff. }}$ The data shown in the Explicit column represent the sensitivity of $k_{\text {eff }}$ to the resonance self-shielded cross sections. The data in the Implicit column represent the additional sensitivity of $k_{\text {eff }}$ introduced by the resonance self-shielding calculation. Data in the Sensitivity column are the sums of the explicit and implicit data and represent the true expected sensitivity of $k_{\text {eff }}$ to changes in the cross section. The values shown in the Sensitivity column of this table are the same values that are used in an energy-dependent format from the .sdf file in TSUNAMI-IP.

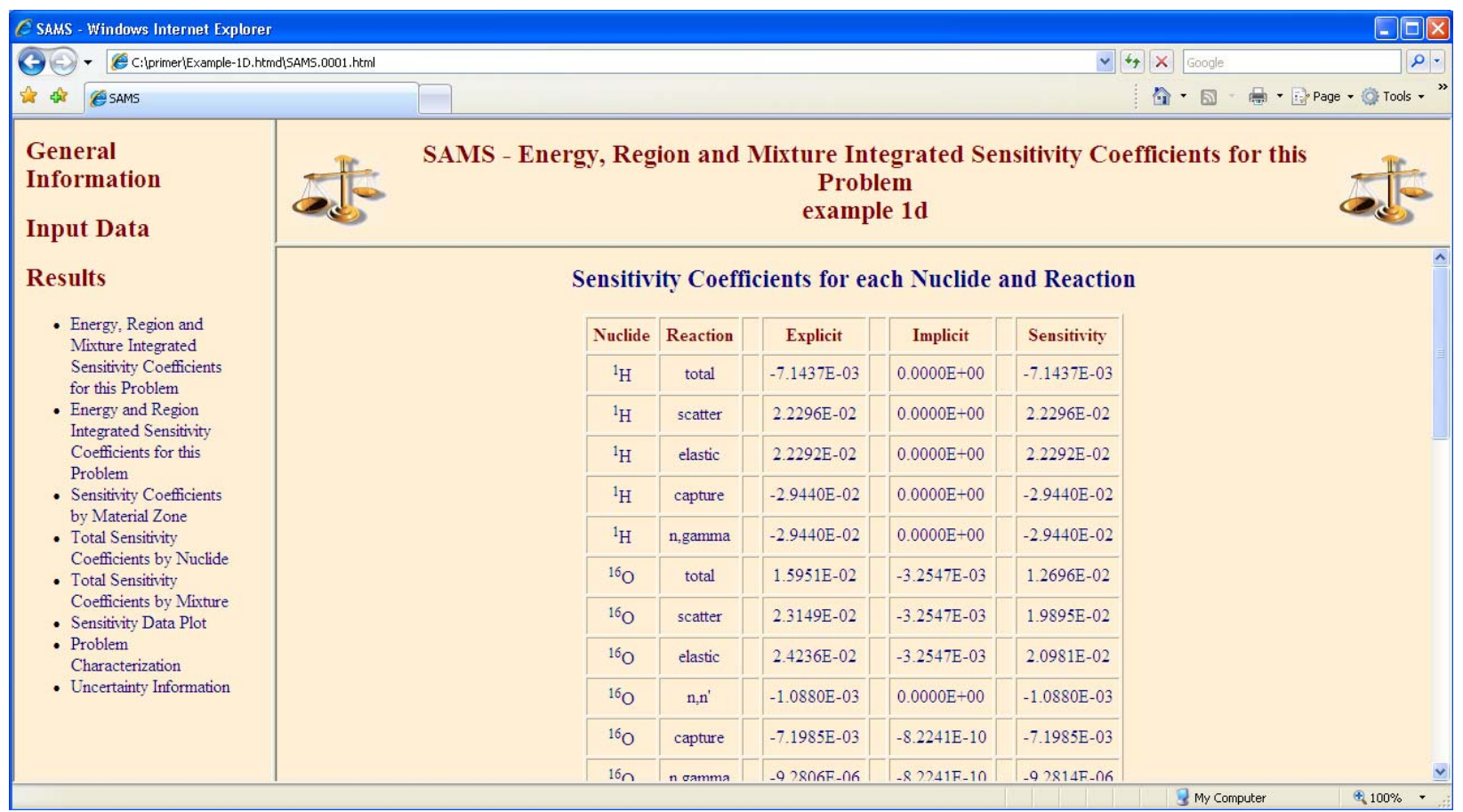

Fig. 15. Energy, region, and mixture integrated sensitivity coefficients in TSUNAMI-1D HTML output.

The HTML output file offers some interactive functionality. You can sort the results in each column in ascending or descending order by clicking on the column heading. An arrow will appear to note the ordering. To return to the original order, simply reload the page. The Nuclide and Reaction tabs will be sorted alphabetically, while the Explicit, Implicit, and Sensitivity tabs will be ordered numerically.

If you scroll through the Results section on the left-side menu, you can see other ways to view the sensitivity data. The next option we will choose is Total Sensitivity Coefficients by Mixture. This option presents data integrated by mixture, so it shows how the system $k_{\text {eff }}$ is sensitive to each mixture, but not specific isotopes or reactions, as shown in Fig. 16. The values shown represent the 
percentage change in $k_{\text {eff }}$ that would be observed by making a $1 \%$ increase in the density of each mixture. Thus, for this problem a $1 \%$ increase in the density of mixture 1 would result in a $0.18722 \%$ increase in $k_{\text {eff. }}$

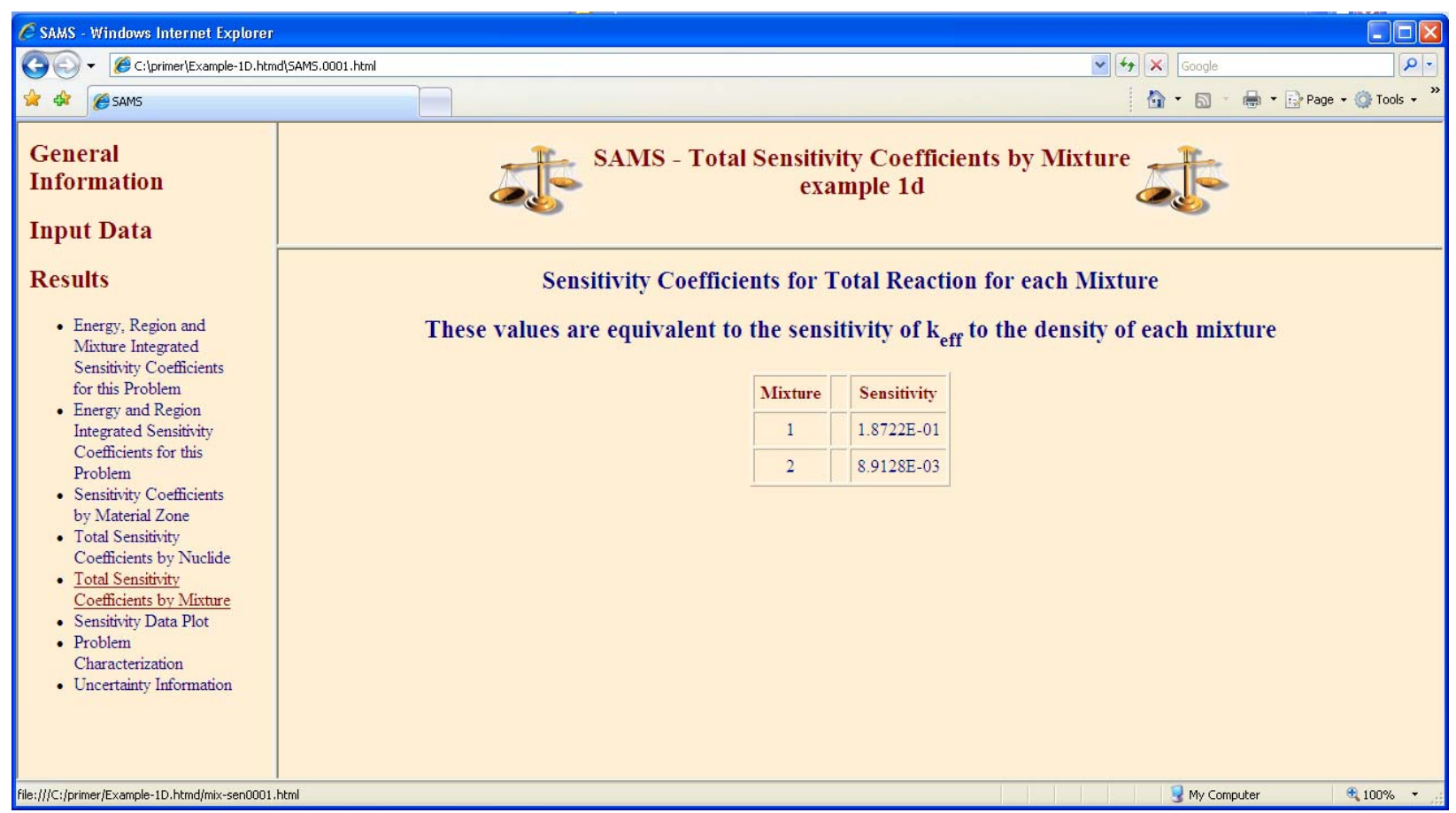

Fig. 16. Total sensitivity coefficients by mixture in HTML output.

To obtain plots of the sensitivity data, select Sensitivity Data Plot from the left menu. A Java applet version of the Javapeño plotting package loads directly in the web browser, as shown in Fig. 17, with all sensitivity data available for plotting. It may be necessary to trust the Javapeño applet, depending on the security settings for your web browser. 


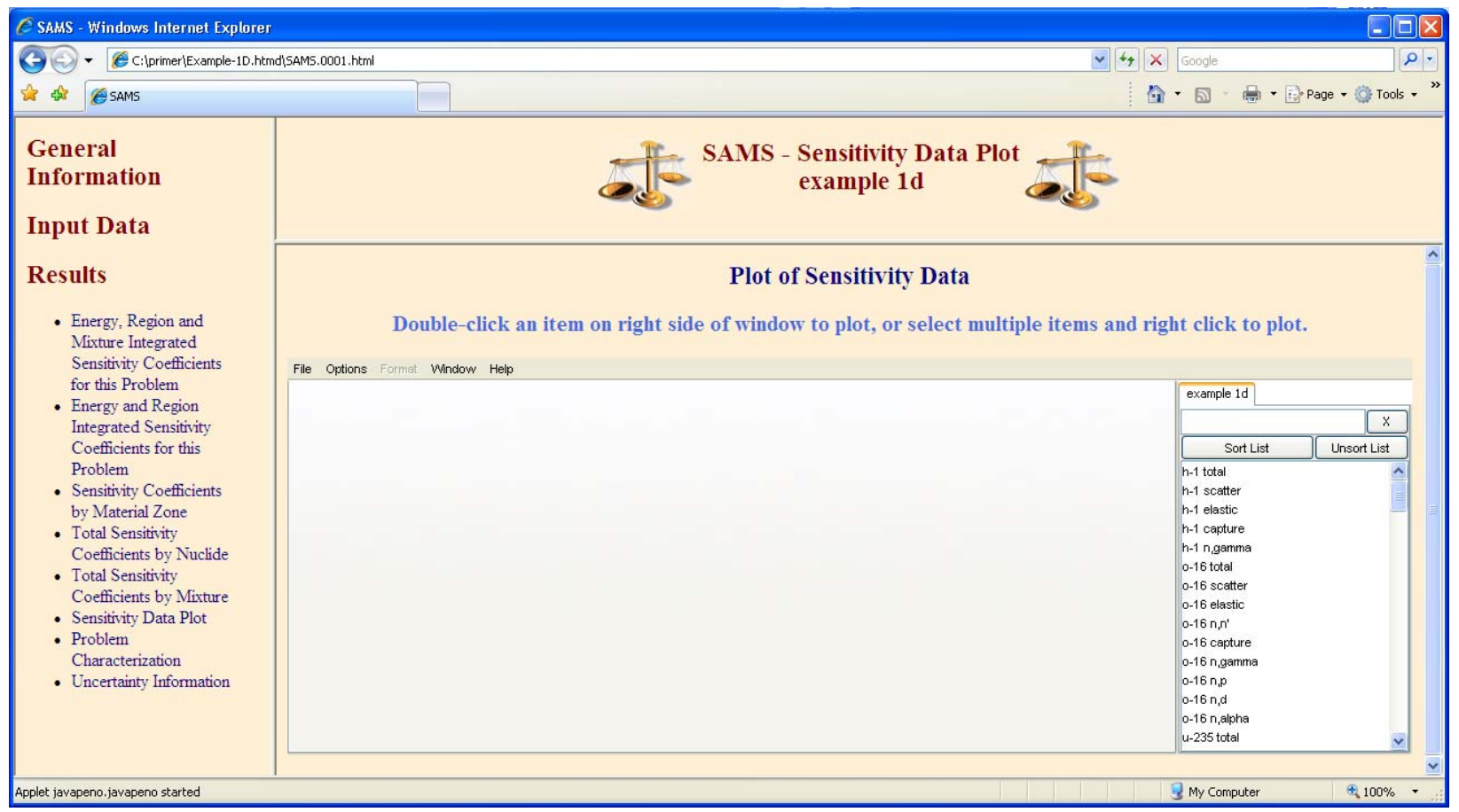

Fig. 17. Sensitivity data plot screen.

A plot of the sensitivity of $k_{\text {eff }}$ to ${ }^{235} \mathrm{U}$ fission cross sections can be displayed by double-clicking u - 235 fission on the right-side menu, as shown in Fig. 18. Note that you can filter the list of data to search for a desired nuclide reaction by typing the desired data in the box at the top of the list, as well as by scrolling through the list. The list can also be sorted in ascending order by clicking on the Sort List button. 


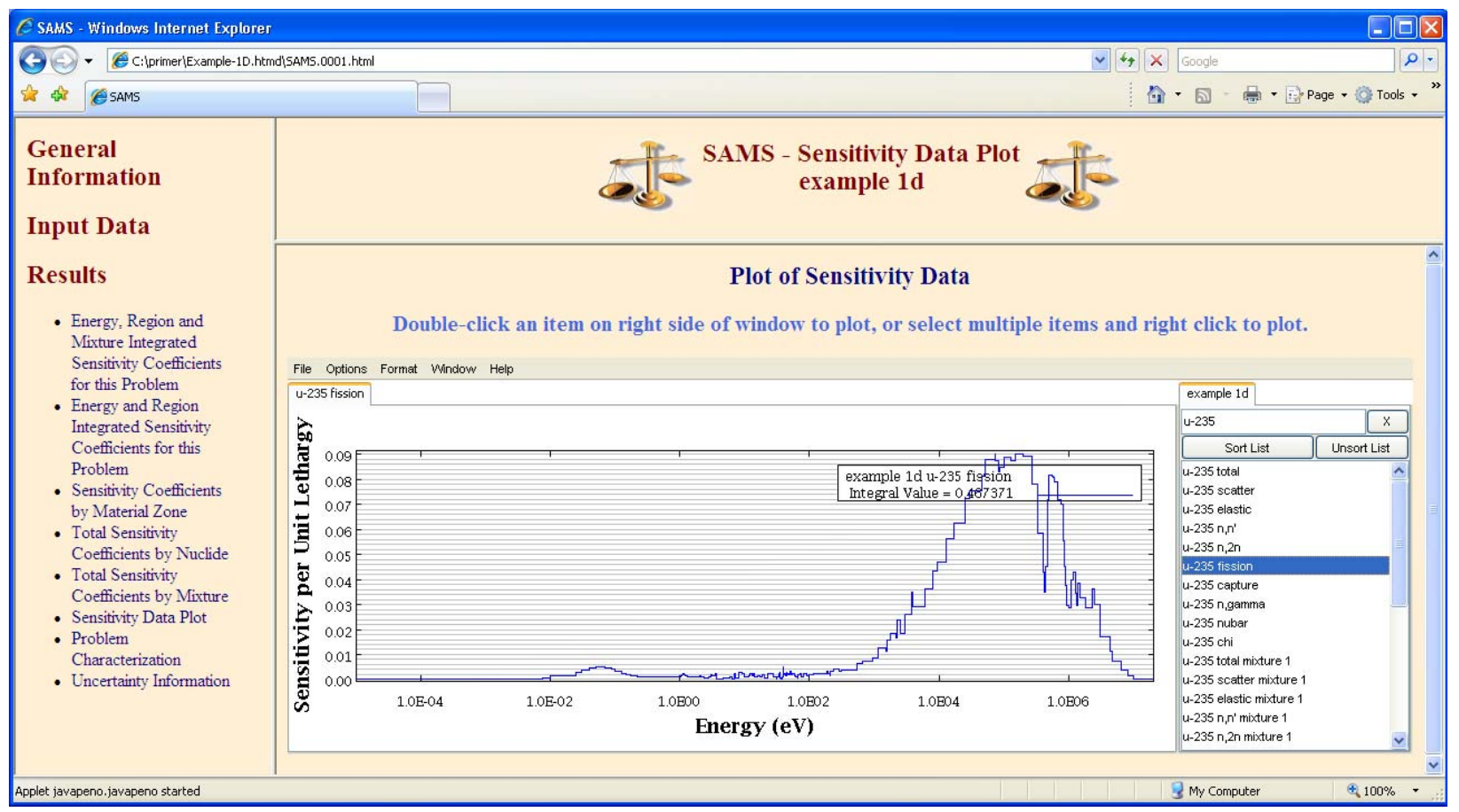

Fig. 18. Sensitivity data plot for "u-235 fission."

The uncertainty in $k_{\text {eff }}$ due to cross-section-covariance data can be viewed in Uncertainty Information, as shown in Fig. 19. For this system, the total uncertainty in $k_{\text {eff }}$ due to uncertainties in the cross-section data is $1.9841 \% \Delta \mathrm{k} / \mathrm{k}$. The contributors to this uncertainty are listed in order of decreasing magnitude in the table.

A more detailed assessment of each output edit is provided in the next section. 


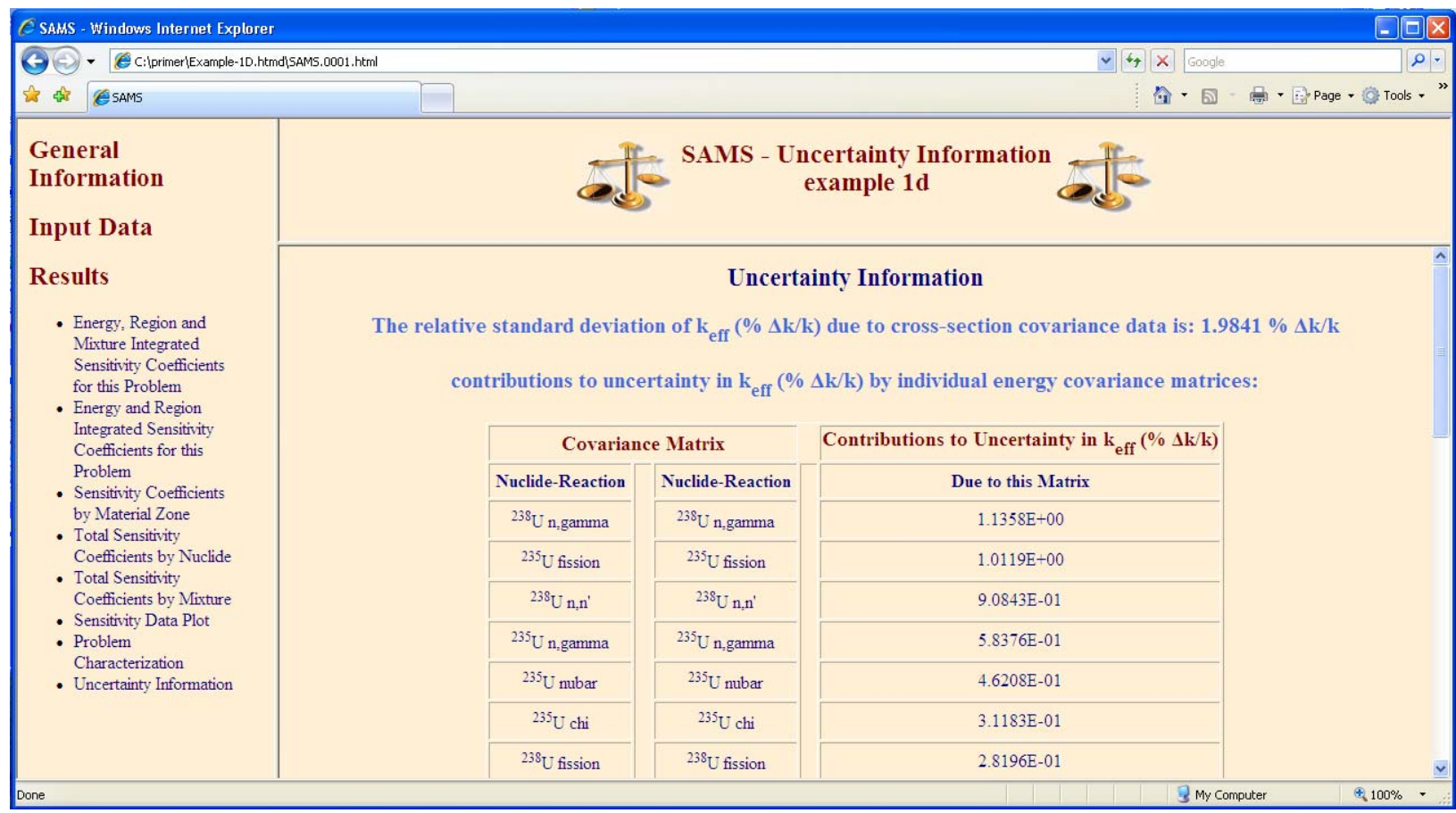

Fig. 19. Uncertainty information for TSUNAMI-1D example problem. 


\section{TSUNAMI-1D OUTPUT}

This section expands on the preliminary explanation of the HTML output provided in Sect. 2.12. There are two ways of viewing the output when using SCALE: as a plain text file or as interactive HTML displayed in a web browser. By default, the SAMS module creates both the text and HTML-formatted output. Because it is a more user-friendly way to examine the output data, we will discuss the HTML output here. The text-based output is described in Sect. F22 of the SCALE Manual.

Beginning with the main screen of the SAMS HTML output shown in Fig. 14, click on General Information and Program Verification Information, which displays the software name and version, the computer name, the date of execution, and the time of execution. You can verify that the correct output file was opened by noting the time and date of execution. If the link Messages appears in the left frame, clicking on it lists any warning or error messages that occurred during the execution of SAMS. Be sure to view any warning or error messages. It is always important to review any messages and assess their potential impact on your results.

Clicking on Input Data, reveals a submenu of data input to the SAMS calculation. The link for Input Parameters, shown in Fig. 20, reveals the input selected in the SAMS data form of GeeWiz. The link Summary of Transport Calculations, shown in Fig. 21, provides a summary of the forward and adjoint transport KENO calculation input parameters and the calculated $k_{\text {eff }}$ values. It is always important to ensure that the forward and adjoint $k_{\text {eff }}$ values closely agree. Any discrepancy between the calculated values indicates that one of the solutions was not correctly created. For TSUNAMI-1D, it is very rare that the forward and adjoint solutions do not agree.

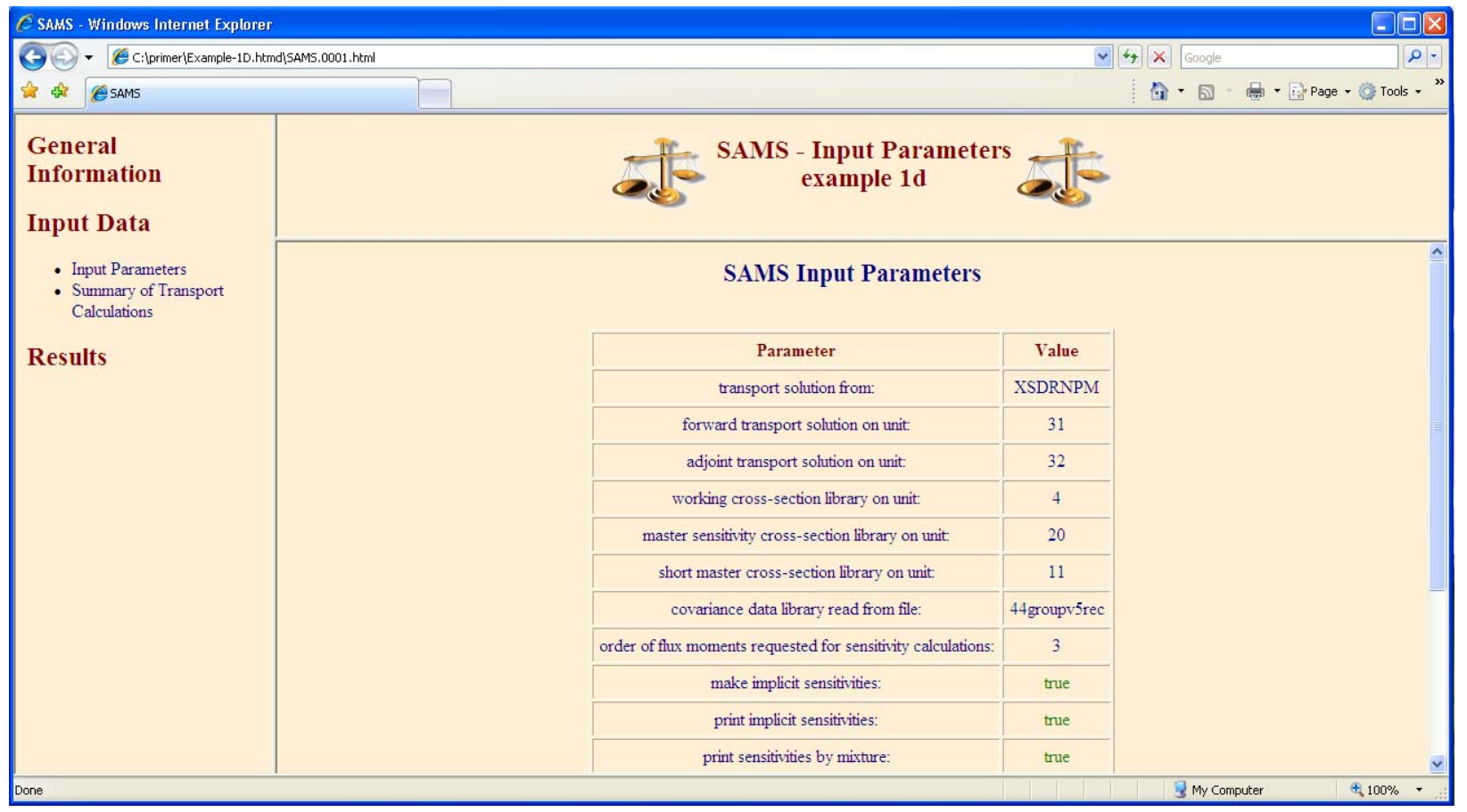

Fig. 20. Input parameters data in HTML output. 


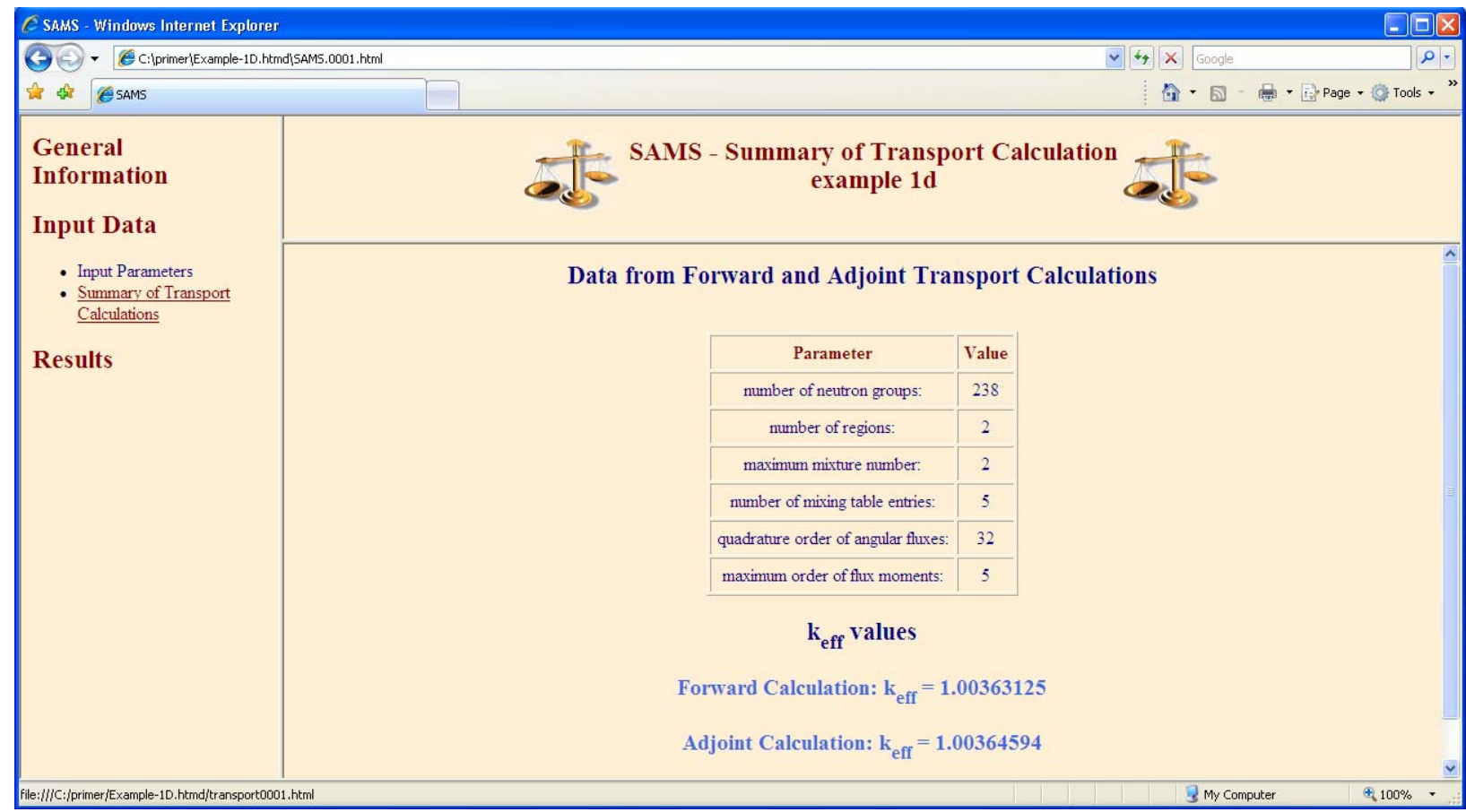

Fig. 21. Summary of transport calculations in HTML output from TSUNAMI-1D.

Clicking on the Results link opens a list of calculated results. Select the link Energy, Region and Mixture Integrated Sensitivity Coefficients for this Problem, which is the first edit list, to display the sensitivities listed by isotope. These are the same results previously shown in Fig. 15. The values presented indicate a percentage change in $k_{\text {eff }}$ that would be observed for a $1 \%$ increase in a given cross section across all energy groups. The data shown in the Explicit column represent the sensitivity of $k_{\text {eff }}$ to the resonance self-shielded cross sections. The data in the Implicit column represent the sensitivity of $k_{\text {eff }}$ introduced by the resonance self-shielding calculation. Data in the Sensitivity column are the sums of the explicit and implicit data and represent the true expected sensitivity of $k_{\text {eff }}$ to changes in the cross section. The values shown in the Sensitivity column of this table are the same values that are used in an energy-dependent format from the .sdf file in TSUNAMI-IP.

Because this is an interactive HTML output file, you may sort the list as desired by clicking on the column headers. By default, the list is sorted by the nuclide's atomic number, then atomic mass, then reaction. The Nuclide and Reaction tabs can be sorted alphabetically, while the Explicit, Implicit, and Sensitivity tabs are ordered numerically; all can be sorted in ascending or descending order with multiple clicks. An arrow will appear to note the ordering. Click on the Sensitivity column twice to sort the data in descending order. That sort order will list total sensitivity coefficients of nuclide reactions beginning with the largest positive sensitivity coefficients, as shown in Fig. 22. Note that clicking once on the Sensitivity column will sort the data so that the largest negative sensitivity coefficients are at the top of the table. To return to the default order, simply reload the page in your web browser.

Fig. 22 shows that this system is most sensitive to ${ }^{235} \mathrm{U}$ nubar, where a $1 \%$ increase in nubar would result in a $0.77757 \%$ increase in $k_{\text {eff }}$. 


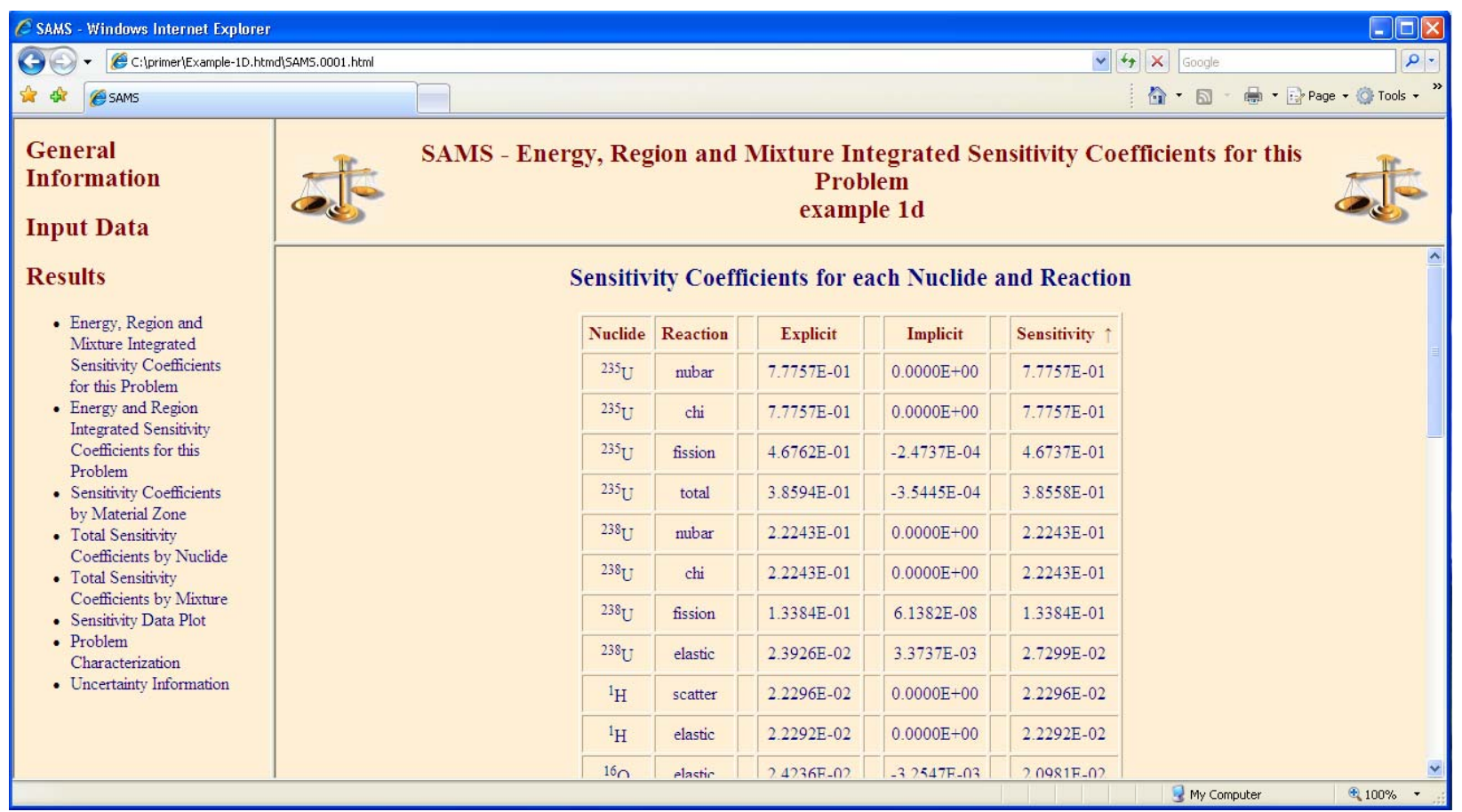

Fig. 22. Energy, region, and mixture integrated sensitivity coefficients in TSUNAMI-1D HTML output sorted by sensitivity in descending order.

The other links under Results in the left frame provide differing ways to view the sensitivity data. The next option, Energy and Region Integrated Sensitivity Coefficients for this Problem, displays the data for each mixture. It is similar to the first edit, but if the same nuclide appears in multiple mixtures, the sensitivity coefficients for that nuclide are distinguished by each mixture in which it occurs. Clicking on the Sensitivity column twice will sort this list in descending order as well. This edit is shown in Fig. 23, where the sensitivity of $k_{\text {eff }}$ to ${ }^{16} \mathrm{O}$ in mixture $1, \mathrm{UO}_{2}$, is distinguished from that for mixture 2, water. Note that even if one of the mixtures was present in more than one geometrical zone, the cumulative sensitivity for all uses of the mixture would be shown here. 


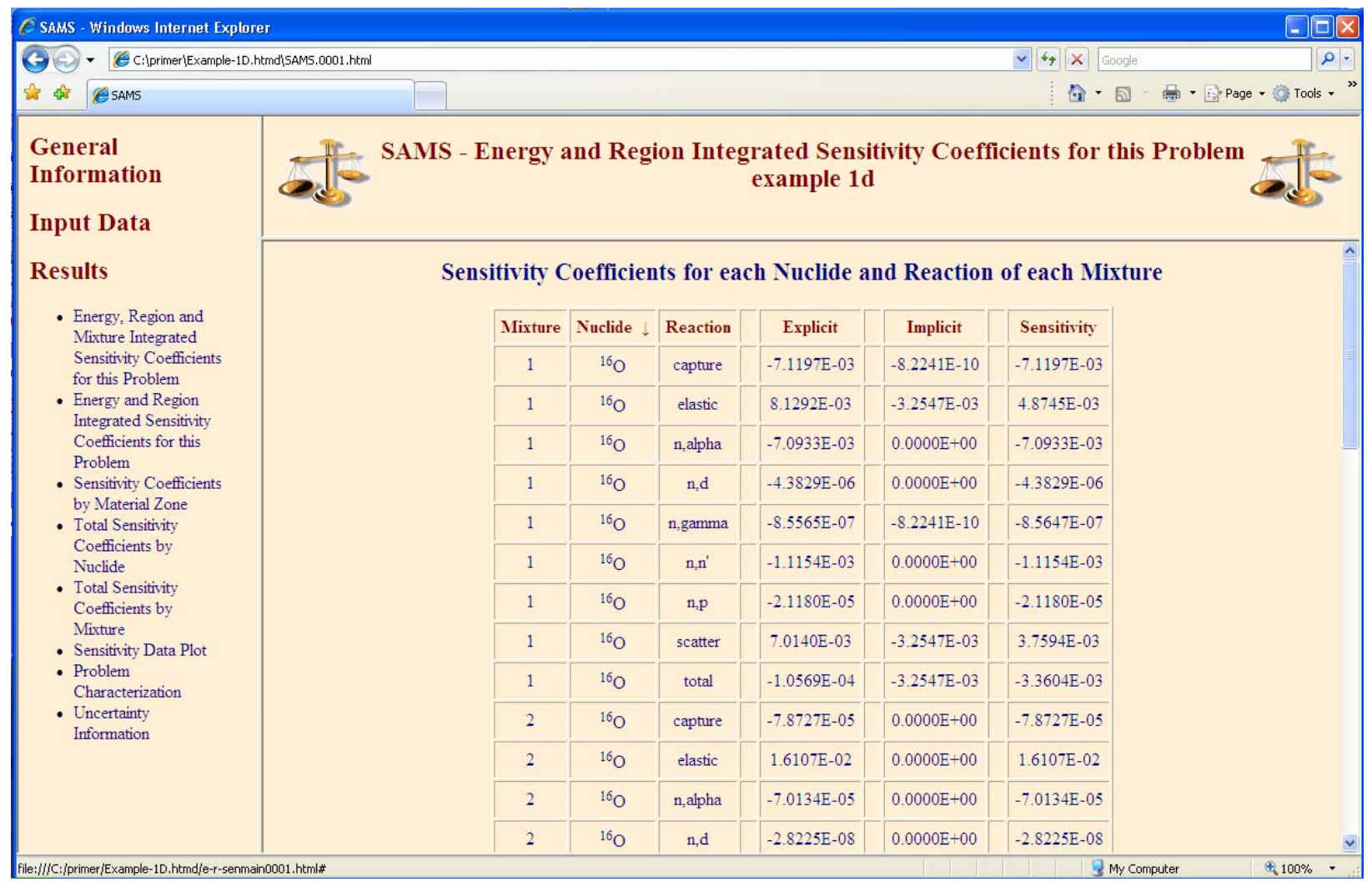

Fig. 23. Energy and region integrated sensitivity coefficients in TSUNAMI-1D HTML output sorted by nuclide in ascending order.

Because the SAMS keyword prtgeom was entered in the input file, the optional edit of Sensitivity Coefficients by Material Zone is next. This edit further distinguishes the sensitivity coefficients by geometrical zone, allowing you to see the sensitivity data by specific nuclide reactions for the mixture present in each zone, as shown in Fig. 24. Note the presence of a second menu on the right side of the screen. This submenu allows you to select the zone for which you wish to view the sensitivity coefficients. We begin by selecting Zone 1 from the list in the right frame. Once again we can sort the sensitivity data by clicking on the column headers. Note that the volume of the zone is also shown, although the sensitivity coefficients are not presented in a per-unit-volume format. 


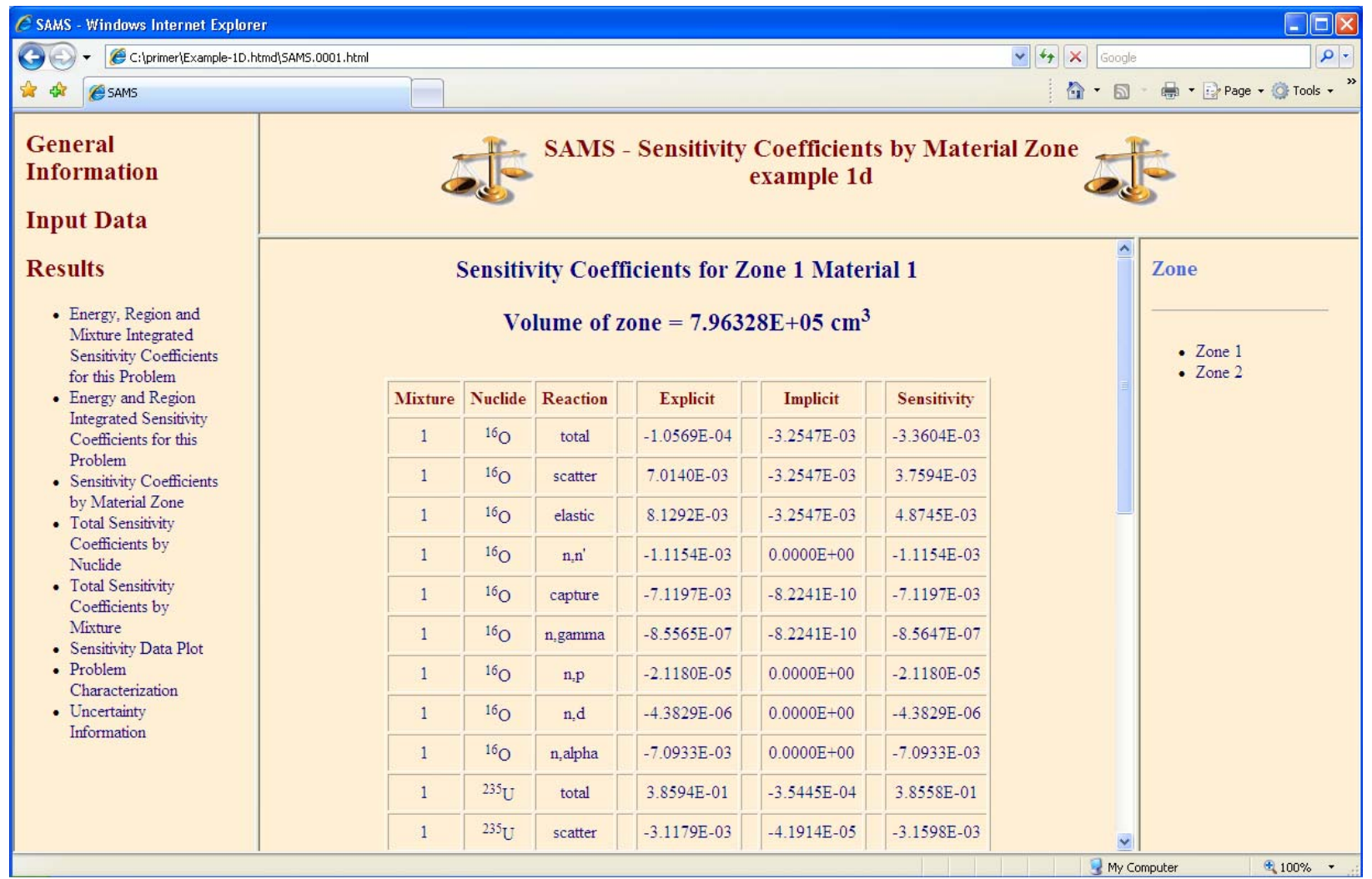

Fig. 24. Sensitivity coefficients by material zone in TSUNAMI-1D HTML output.

The next output edit, Total Sensitivity Coefficients by Nuclide, shows only the sensitivity data for the total reactions by nuclide and mixture. The results shown in this table are equivalent to sensitivity of $k_{\text {eff }}$ to the density of the nuclide in the mixture, so this table also includes the atomic density of each nuclide, as shown in Fig. 25. 


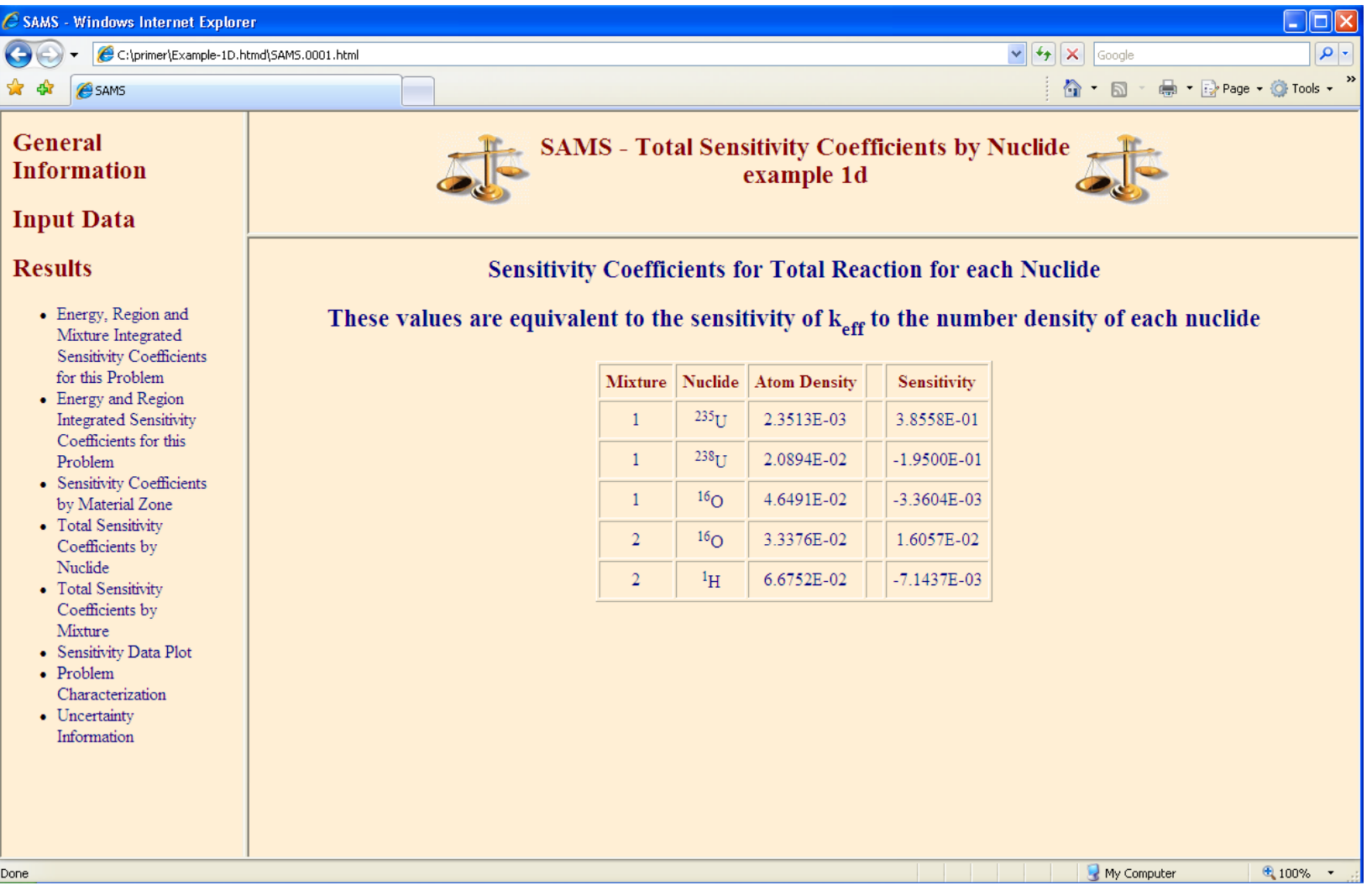

Fig. 25. Sensitivity coefficients by nuclide in TSUNAMI-1D HTML output.

The next edit is Total Sensitivity Coefficients by Mixture, where the sensitivity data are summed over all nuclides in a mixture so they show how the system $k_{\text {eff }}$ would respond to changes in the mixture density, as shown in Fig. 26. 


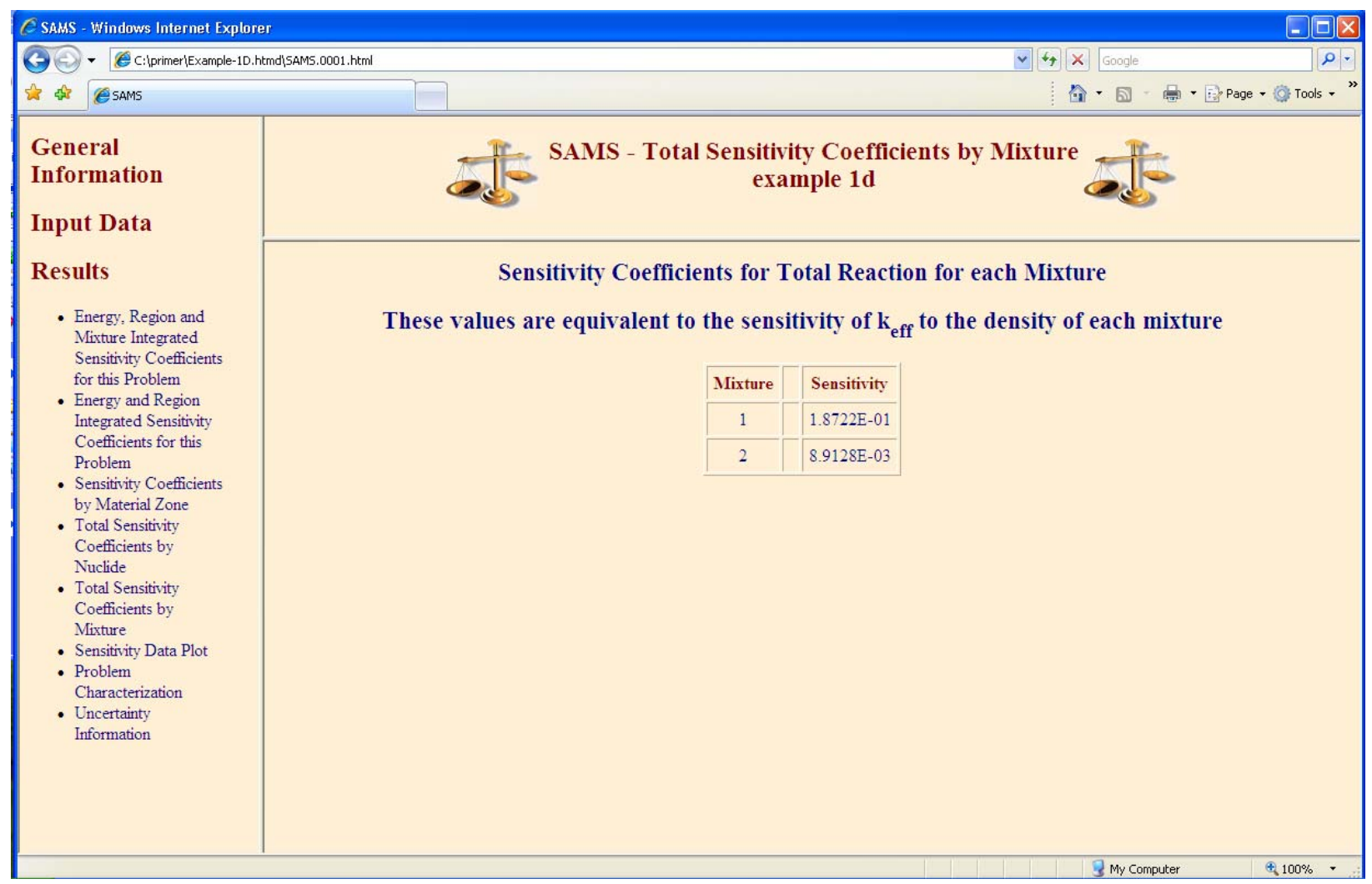

Fig. 26. Total sensitivity coefficients by mixture in TSUNAMI-1D HTML output.

The link for Sensitivity Data Plot opens a Javapeño applet inside the HTML output in which you can plot energy-dependent data by nuclide and reaction; nuclide, reaction, and mixture; and nuclide, reaction and zone by double-clicking items on the right-hand list. Note that you can filter the list of data to search for a desired nuclide reaction by typing the desired data in the box at the top of the list as well as by scrolling through the list. The list can also be sorted in ascending order by clicking on the Sort List button. It may be necessary to trust the Javapeño applet, depending on the security settings for your web browser. The HTML output with the plot ready for use is shown in Fig. 17.

A sample plot is shown in Fig. 27, where the sensitivity of $k_{\text {eff }}$ to ${ }^{16} \mathrm{O}$ elastic scattering in zones 1 and 2 is shown. You can generate this plot with the following steps. First, type $0-16$ in the filter field just above the data list on the right side of the plot. This eliminates all data except for those for ${ }^{16} \mathrm{O}$. Next, scroll down the list until you see 0-16 elastic zone 1 . This is the sensitivity of $k_{\text {eff }}$ to ${ }^{16} \mathrm{O}$ elastic scattering in zone 1. Double-click this item in the list to add it to the plot. Next, continue scrolling until you see 0-16 elastic zone 2. This is the sensitivity of $k_{\text {eff }}$ to ${ }^{16} \mathrm{O}$ elastic scattering in zone 2 . Double-click this item to add it to the plot. You should now have a plot just like that shown in Fig. 27. 


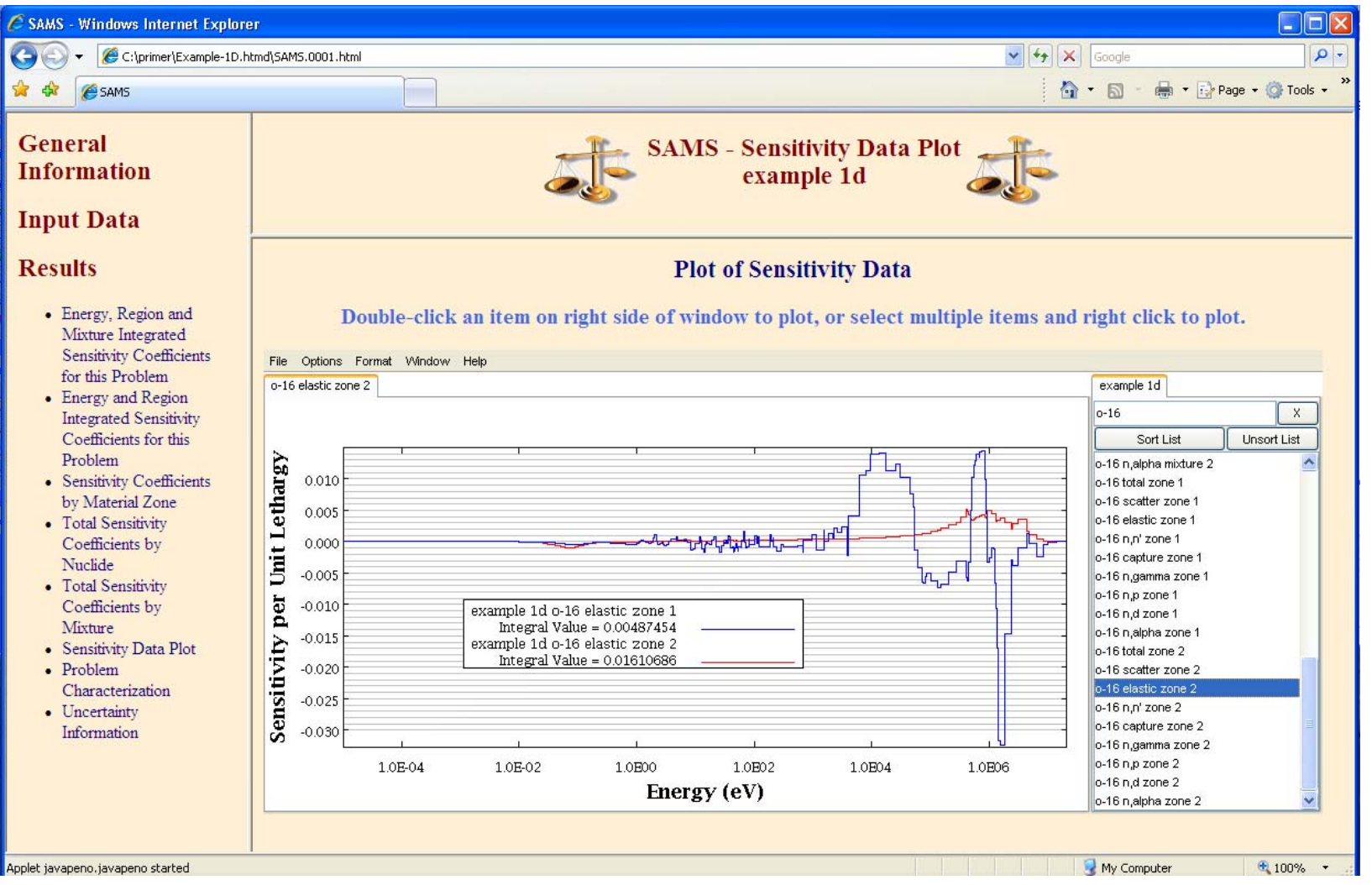

Fig. 27. Sensitivity data plot in TSUNAMI-1D HTML output with ${ }^{16} \mathrm{O}$ elastic scattering sensitivities for zones 1 and 2.

The edit of Problem Characterization shows the average fission, capture, and scattering parameters for the forward calculation of the model, as shown in Fig. 28. Although this is not a sensitivity or uncertainty edit, it is shown here because SAMS has access to all required data for these edits, and these data are often useful in code validation, a primary function of TSUNAMI. 


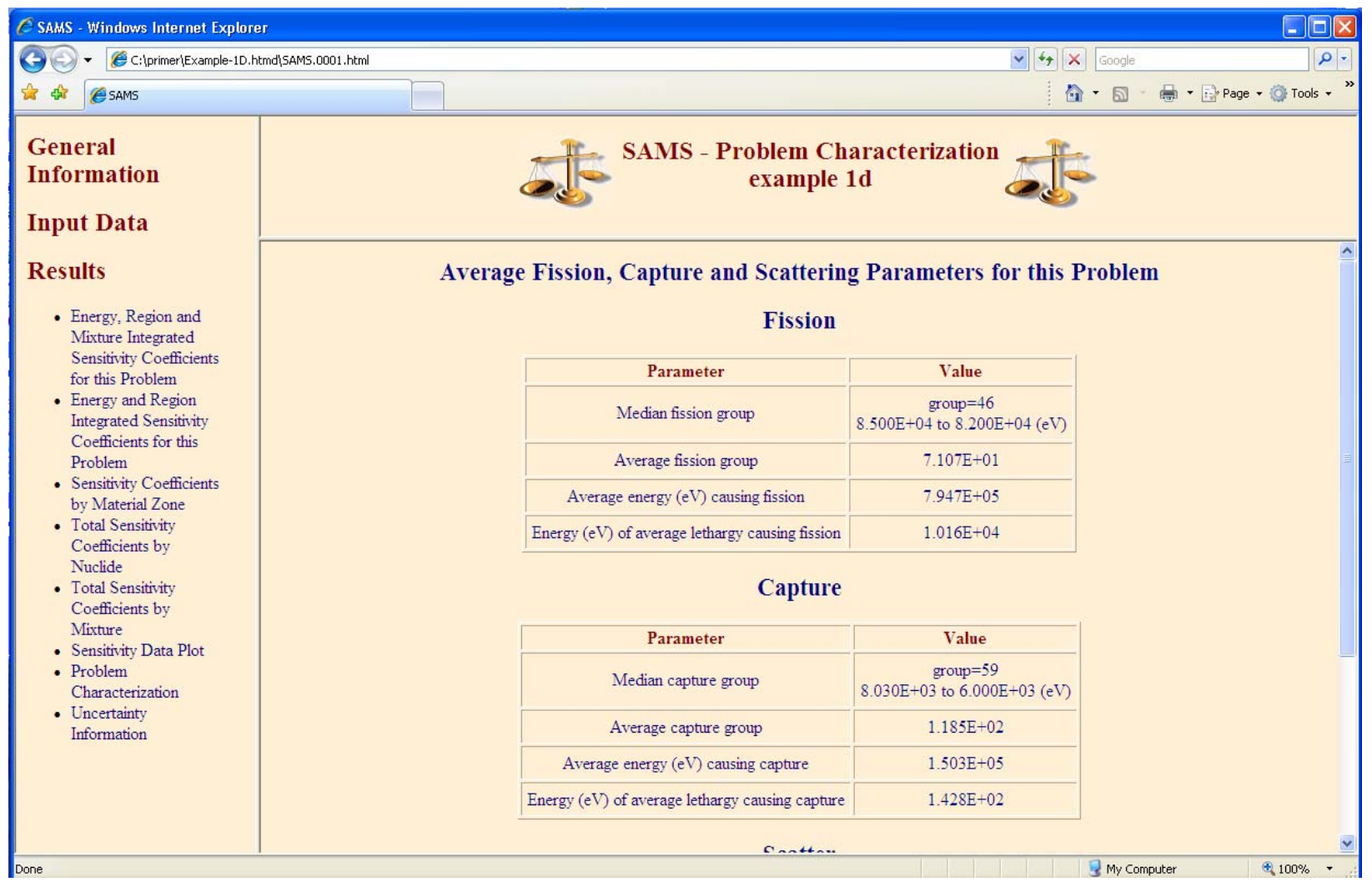

Fig. 28. Problem characterization in TSUNAMI-1D HTML output.

The uncertainty in $k_{\text {eff }}$ due to cross-section covariance can be viewed in Uncertainty Information, as shown in Fig. 19, with additional data shown in Fig. 29. For this system, the total uncertainty in $k_{\text {eff }}$ due to uncertainties in the cross-section data is $1.9841 \% \Delta \mathrm{k} / \mathrm{k}$. The contributors to this uncertainty are listed in order of decreasing magnitude in the table in unit of percent $\Delta \mathrm{k} / \mathrm{k}$ for each covariance matrix.

A covariance matrix describes not only the uncertainty in a particular value, the variance, but also the shared uncertainty between different values, the covariance. In cross-section-covariance data, the uncertainty is tabulated as a function of energy for a nuclide reaction to itself or to another nuclide reaction. Thus, the table of uncertainty data shown in Fig. 29 describes what two nuclide reactions are included in each covariance matrix. Often, the covariance matrix details the uncertainties of a nuclide reaction and the shared uncertainties for the same nuclide reaction at different energies. In this case, the covariance matrix is identified with the same nuclide and reaction in both columns. In other cases, the shared uncertainties between different reactions of the same nuclide or even between different nuclides are tabulated in the nuclear data libraries. In these cases, the identifiers in each column are different.

The values shown in this output edit are derived from the covariance values but expressed in a more familiar $\Delta \mathrm{k} / \mathrm{k}$ format for the convenience of the user. The total uncertainty can be computed from individual values by adding the square of the values with positive signs and subtracting the square of the values with negative signs, then taking the square root. The negative values are the result of anticorrelations in the cross-section-covariance data. A negative covariance results from a common uncertainty between two components and actually reduces the overall uncertainty that could have been observed if the individual uncertainties were not related. 


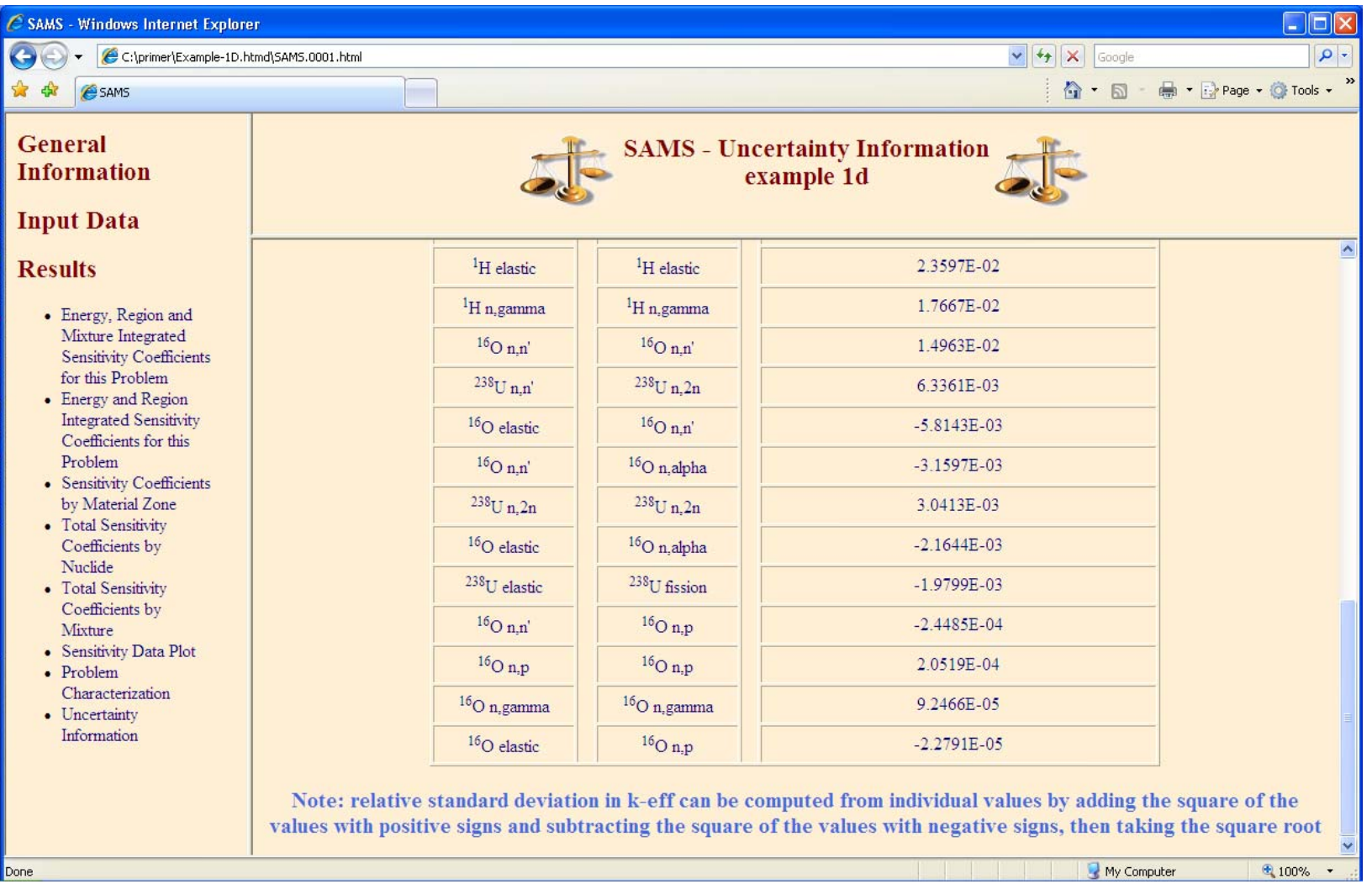

Fig. 29. Uncertainty information in TSUNAMI-1D HTML output. 


\section{DIRECT PERTURBATION CALCULATIONS}

The user must ensure that the necessary options are employed for each model to obtain accurate results. The validity of the energy-integrated sensitivity coefficients can be confirmed through the use of central difference direct perturbation sensitivity calculations. Through this technique, the sensitivity of $k_{\text {eff }}$ to the number density of a particular nuclide can be obtained. This sensitivity of $k_{\text {eff }}$ to the number density is equivalent to the sensitivity of $k_{\text {eff }}$ to the total cross section, integrated over energy. Because the total cross-section sensitivity coefficient tests much of the data used to compute all other sensitivity coefficients, it is considered an adequate test for verification. For each sensitivity coefficient examined by direct perturbation, the $k_{\text {eff }}$ of the system is computed first with the nominal values of the input quantities, then with a selected nominal input value increased by a certain percentage, and then with the nominal value decreased by the same percentage. The direct perturbation sensitivity coefficient of $k_{\text {eff }}$ to some input value $\alpha$ is computed as

$$
S_{k, \alpha}=\frac{\alpha}{k} \times \frac{d k}{d \alpha}=\frac{\alpha}{k} \times \frac{k_{\alpha^{+}}-k_{\alpha^{-}}}{\alpha^{+}-\alpha^{-}}
$$

where $\alpha^{+}$and $\alpha^{-}$represent the increased and decreased values, respectively, of the input quantity $\alpha$, and $k_{\alpha^{+}}$and $k_{\alpha^{-}}$represent the corresponding values of $k_{\text {eff. }}$ When direct perturbation calculations are performed using KENO, the Monte Carlo statistical uncertainties in the computed values of $k_{\text {eff }}$ are propagated to uncertainties in direct perturbation sensitivity coefficients by standard error propagation techniques as 7

$$
\sigma_{S}=\left(\left(\frac{\left(\sigma_{k^{+}}^{2}+\sigma_{k^{-}}^{2}\right)}{\left(k^{+}-k^{-}\right)^{2}}+\frac{\sigma_{k}^{2}}{k^{2}}\right) \times\left(\frac{k^{+}-k^{-}}{k}\right)^{2}\right)^{1 / 2} \times \frac{\alpha}{\alpha^{+}-\alpha^{-}} .
$$

It is important in sensitivity calculations to ensure that the $k_{\text {eff }}$ value of the forward and adjoint solutions closely agree. If the $k_{\text {eff }}$ values do not agree, then the quality of at least one of the transport calculations may be in question. Typically, the transport calculation of concern is the adjoint calculation.

To calculate total sensitivities using direct perturbation, the user selects which nuclide or mixture to modify, determines how much to modify the density of the selected nuclide or mixture, runs the 1D or 3D calculations, and then uses the equations presented earlier in this section to calculate the total sensitivity.

The user could choose to verify the total sensitivity for each nuclide in each zone; however, this is generally not necessary. The goal is to verify that the forward and adjoint calculations are adequately converged and that the forward and adjoint solutions contain adequate spatial and angular resolution. This may be accomplished by testing a nuclide or a mixture in one or more specific regions of a problem. For example, in a fuel storage rack model, one might check the total sensitivity of ${ }^{235} \mathrm{U}$ in the fuel pellet region, the $\mathrm{H}_{2} \mathrm{O}$ around the fuel pins, and the steel in the storage rack cell wells. This would ensure that the forward and adjoint solutions were acceptable in the fuel pins, the fuel pin moderator, and the rack structure. One could also check the sensitivity of the ${ }^{238} \mathrm{U}$ in the fuel pellet region, but this is not necessary.

The size of the perturbation varies first with whether the TSUNAMI calculation is a 1D or 3D calculation. Verification of a 1D calculation may be performed with relatively small perturbations, typically $1 \%$ or less. The only concern here is that the behavior of $k_{e f f}$ should be linear over the range of the perturbation. 
If a $1 \%$ perturbation is used, the $-1 \%$, nominal, and $+1 \% k_{\text {eff }}$ values should fall very close to a straight line. Because the $1 \mathrm{D}$ convergence criterion is typically smaller than $1 \times 10^{-5} \Delta \mathrm{k}$, the $k_{\text {eff }}$ values on the plus and minus sides should vary by at least $1 \times 10^{-4} \Delta \mathrm{k}$.

Verification of a 3D calculation is a little more complicated because the Monte Carlo statistical uncertainty is typically larger, and consequently, the variation in $k_{e f f}$ due to minor density changes may not be visible. A rule of thumb for $3 \mathrm{D}$ calculations is that the density perturbation should be large enough so that the high- and low-end $k_{\text {eff }}$ values differ by about 10 standard deviations. For example, if the total sensitivity for a nuclide has been calculated to be $-0.1(\Delta \mathrm{k} / \mathrm{k}) /(\Delta \sigma / \sigma)$, we are willing to run our direct perturbation KENO calculations to a standard deviation of $0.0002 \Delta \mathrm{k}$, and the model has a nominal $k_{\text {eff }}$ value of 0.9 , these data and the definition of sensitivity may be used to calculate the size of the perturbation needed to yield a 10 -standard-deviation spread in the $k_{\text {eff }}$ values. The definition of sensitivity is

$$
S=\frac{\Delta k / k}{\Delta \sigma / \sigma} .
$$

The total fractional density change from high to low is then

$$
\Delta \beta / \beta=\Delta \sigma / \sigma=\frac{1}{s}, \Delta k / k
$$

The fractional density change required to yield 5 standard deviations' difference between the nominal case and the high and low sides is then

$$
\Delta \beta / \beta=\frac{1}{0.1} ; 5: 0.0002 / 0.9=0.011
$$

Thus, if the density of the selected nuclide or mixture is varied $\pm 1.1 \%$ from the nominal, the high and

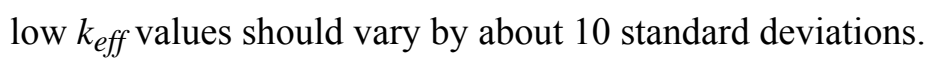

As with the 1D direct perturbation calculations, it is important that the perturbations not be too large. The behavior of $k_{\text {eff }}$ between the high and low perturbations should be nearly linear. When doing 3D calculations, in some cases it may not be possible to meet both the linear behavior and 10-standarddeviation spread criteria. In such cases, it may be necessary to use more sophisticated techniques or to accept a qualitative verification.

As a more concrete example of using direct perturbation calculations to verify TSUNAMI-3D sensitivity results, the following direct perturbation calculation is provided for the model used as an example in Sect. 7 of this document.

The sensitivity of $k_{\text {eff }}$ to variation of the ${ }^{233} \mathrm{U}$ total cross section was calculated to be $0.11471 \pm 4.2 \times 10^{-4}$, and the $k_{\text {eff }}$ value for this model is $1.00262 \pm 1.9 \times 10^{-4}$. 
When the direct perturbation calculations will be run to a Monte Carlo standard deviation of about 0.0002 , the size of the ${ }^{233} \mathrm{U}$ density perturbation needed to yield a 10 -standard-deviation spread in $k_{\text {eff }}$ values is calculated as follows:

$$
\Delta \beta / \beta=\frac{1}{0.11471} * 0.0002 / 1.00262=0.0087 .
$$

CSAS25 direct perturbation calculations are then performed for the nominal case and for $\pm 0.9 \% \Delta \rho / \rho$, yielding the following results:

$$
\begin{aligned}
& k_{\text {eff }}\left(\rho=0.991 * \rho_{\text {nom }}\right)=1.00156 \pm 0.00019, \\
& k_{\text {eff }}\left(\rho=1.000 * \rho_{\text {nom }}\right)=1.00262 \pm 0.00019, \\
& k_{\text {eff }}\left(\rho=1.009 * \rho_{\text {nom }}\right)=1.00374 \pm 0.00020 .
\end{aligned}
$$

The high-to-low $k_{\text {eff }}$ range is then an acceptable 11 standard deviations.

$$
s=\frac{(1.00374-1.00156) / 1.00262}{(1.009-0.991) / 1.000}=0.1208
$$

The uncertainty on the direct perturbation sensitivity is calculated as shown below.

$$
\begin{aligned}
\sigma_{s} & =\left(\left(\frac{\left(0.0002^{2}+0.00019^{2}\right)}{(1.00374-1.00156)^{2}}+\frac{0.00019^{2}}{1.00262^{2}}\right) *\left(\frac{(1.00374-1.00156)}{1.00262}\right)^{2}\right)^{1 / 2} \\
\frac{1.000}{1.009-0.991} & \\
\sigma_{S} & =0.0153
\end{aligned}
$$

The ${ }^{233} \mathrm{U}$ total sensitivity calculated using the direct perturbation method is then $0.1208 \pm 0.0153(13 \%)$. The sensitivity calculated by TSUNAMI was $0.11471 \pm 4.2 \times 10^{-4}(0.4 \%)$. The TSUNAMI-calculated sensitivity value differs from the direct perturbation sensitivity value and by about 0.4 standard deviations. Anything less than 2 standard deviations is considered acceptable.

Once direct perturbations have been used to verify the sensitivities for a model, it is not necessary to test the sensitivities of minor variations to the checked model. For example, it may be adequate to test a single critical configuration model in a critical experiment series if the model variations are not too extreme. 



\section{UNIT CELL CALCULATIONS FOR TSUNAMI}

The sensitivity data values calculated by TSUNAMI-3D and -1D are comprised of an explicit component and an implicit component. The explicit component is the part of the sensitivity that is due to how the system multiplication factor changes when the related multigroup cross sections change. The implicit component is the part of the sensitivity related to how the resonance self-shielding calculations affect the multigroup cross sections. For example in a water-moderated system, the generation of the self-shielded ${ }^{238} \mathrm{U}$ capture cross sections may be significantly affected by hydrogen scattering. In such a case, the ${ }^{238} \mathrm{U}$ capture reaction sensitivity includes explicit contributions due to sensitivity of the system $k_{\text {eff }}$ value to variation in the ${ }^{238} \mathrm{U}$ capture cross sections and an implicit component due to how the hydrogen scattering affects the resonance self-shielding calculations. The implicit effect can, in some cases, be a major contributor to sensitivity. Accurately capturing the implicit effect requires use of good unit cell models in the TSUNAMI-3D and -1D calculations. Poor unit cell modeling is frequently the reason TSUNAMIcalculated sensitivities and direct perturbation results disagree.

Unit cell modeling is described in Sect. M7, "The Material Information Processor for SCALE," of the SCALE Manual. The guiding principle is to try to perform unit cell modeling of each material in a way that is similar to how the material is used in the transport calculation. If the model is of fuel pins in a lattice, use the appropriate lattice cell modeling described in Sects. M.7.2.4.2 and M7.3.6 of the SCALE Manual. In some cases reflecting materials can have a significant impact on the sensitivities of the nearby resonance absorbers. Capturing these effects in 1D unit cell models can be challenging. Note that the default unit cell treatment is an infinite, homogeneous material (see SCALE Manual Sect. M7.2.4.1). In some cases the infinite, homogeneous approximation may not be appropriate.

The first indication that the unit cell modeling may not be adequate will be that the direct perturbation calculation results disagree with the TSUNAMI-calculated values. If the discrepancy is due to a unit cell modeling problem, the user may be able to identify which nuclide is causing the problem by doing direct perturbation calculations for specific nuclides and by using the Javapeño data visualization program to view the detailed nuclide-, reaction-, and energy-dependent sensitivity data. Very large spikes in one energy group, as shown in Fig. 30, may indicate a unit cell modeling problem. Another indication might be SAMS warning messages indicating a very large implicit contribution.

In TSUNAMI-1D calculations, it is usually appropriate to use a multiregion unit cell model that is identical to the geometry model. The primary exception to this rule is when the $1 \mathrm{D}$ model is used with reflective boundary conditions meant to approximate an array. In this case, a lattice cell unit cell model should be used.

One-dimensional unit cell modeling for TSUNAMI-3D models can be considerably more challenging. A fuel transport cask model would likely require one or more lattice cell treatments for the fuel pins, a multiregion slab cell treatment fuel assembly basket structure that may include neutron absorber panels, and an infinite homogeneous cell treatment for the thick steel cask body.

If there is no evidence of problems with the implicit sensitivity, the user should explore whether the appropriate TSUNAMI-3D analysis parameters have been used, such as collecting high enough order flux moments (see the pnm parameter) or using fine enough mesh flux accumulators (see the $\mathrm{mfx}$ and msh parameters). 


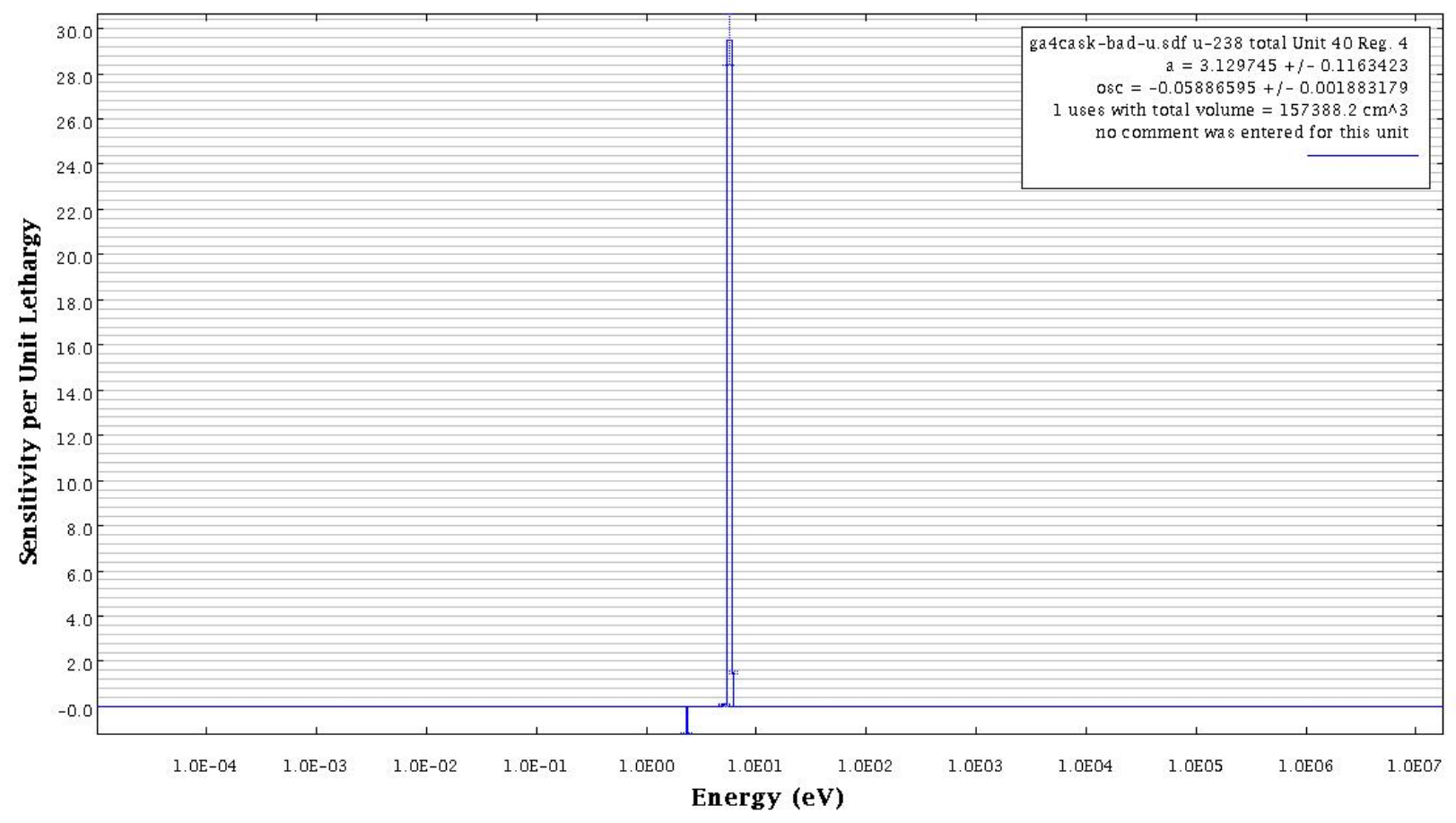

Fig. 30. Example of sensitivity profile indicating a unit cell modeling problem. 


\section{FISSION SPECTRUM (CHI) SENSITIVITY CALCULATIONS}

Based on recent examinations by ORNL staff, as bolstered by the findings of the Organisation for Economic Co-operation and Development/Nuclear Energy Agency Working Party on International Data Evaluation Co-operation Subgroup 26, the fission spectrum sensitivities computed using the classical approach implemented in TSUNAMI in SCALE 5.0 and 5.1 do not consider the normalization of the fission spectrum to unity when computing groupwise chi sensitivities. Fission spectrum data are different from other nuclear data because, by definition, the fission spectrum for a given nuclide must integrate to one. The classical approach to calculating chi sensitivity coefficients ignores this normalization such that the sensitivity to each group is computed independent of the offsetting effects that would be observed in preserving the normalization. In uncertainty calculations, the chi covariance data generated for SCALE does account for the anticorrelations in the data-if one value is increased, others must be decreased. However, it has not been proven that this covariance treatment is always equivalent to preserving the normalization with the sensitivity data. Increasing chi in one energy group without adjusting the others is equivalent to adjusting nubar, which is already considered separately in the calculation of the nubar sensitivities. Errors in the shape of the chi distribution could contribute to the system bias, but the treatment in TSUNAMI in SCALE 5.0 and 5.1 exaggerated the importance of chi for some systems. Due to compensating adjustments, small variations in the shape of the chi distribution should have little effect on $k_{\text {eff. }}$. Differences in the uncertainty in $k_{\text {eff }}$ for a single system based on constrained or unconstrained chi are small due to the anticorrelations present in the cross-section-covariance data for chi. The TSUNAMI techniques for SCALE 6 were modified so fission spectrum sensitivities are produced in a constrained calculation that is equivalent to preserving the requirement that chi data itself integrate to unity. The chi sensitivities for the TSUNAMI-1D1 sample problem, a homogeneous system of 2 -wt- $\%$-enriched $\mathrm{UF}_{4}$ and paraffin, computed with SCALE 5.1 and SCALE 6 are shown in Fig. 31. Here the integral value shown in the legend is the integral of the groupwise sensitivity coefficients. With SCALE 5.1, when summed over all nuclides, the sensitivity of $k_{\text {eff }}$ to chi should equal 1.0. With SCALE 6, the same sum should be nearly 0.0. In Fig. 31, the integral values for the ${ }^{235} \mathrm{U}$ chi sensitivities are 0.95 and nearly 0.0 for SCALE 5.1 and SCALE 6, respectively. The integral values for chi sensitivities for ${ }^{238} \mathrm{U}$, not shown, are 0.05 and nearly 0.0 for SCALE 5.1 and SCALE 6 , respectively. 


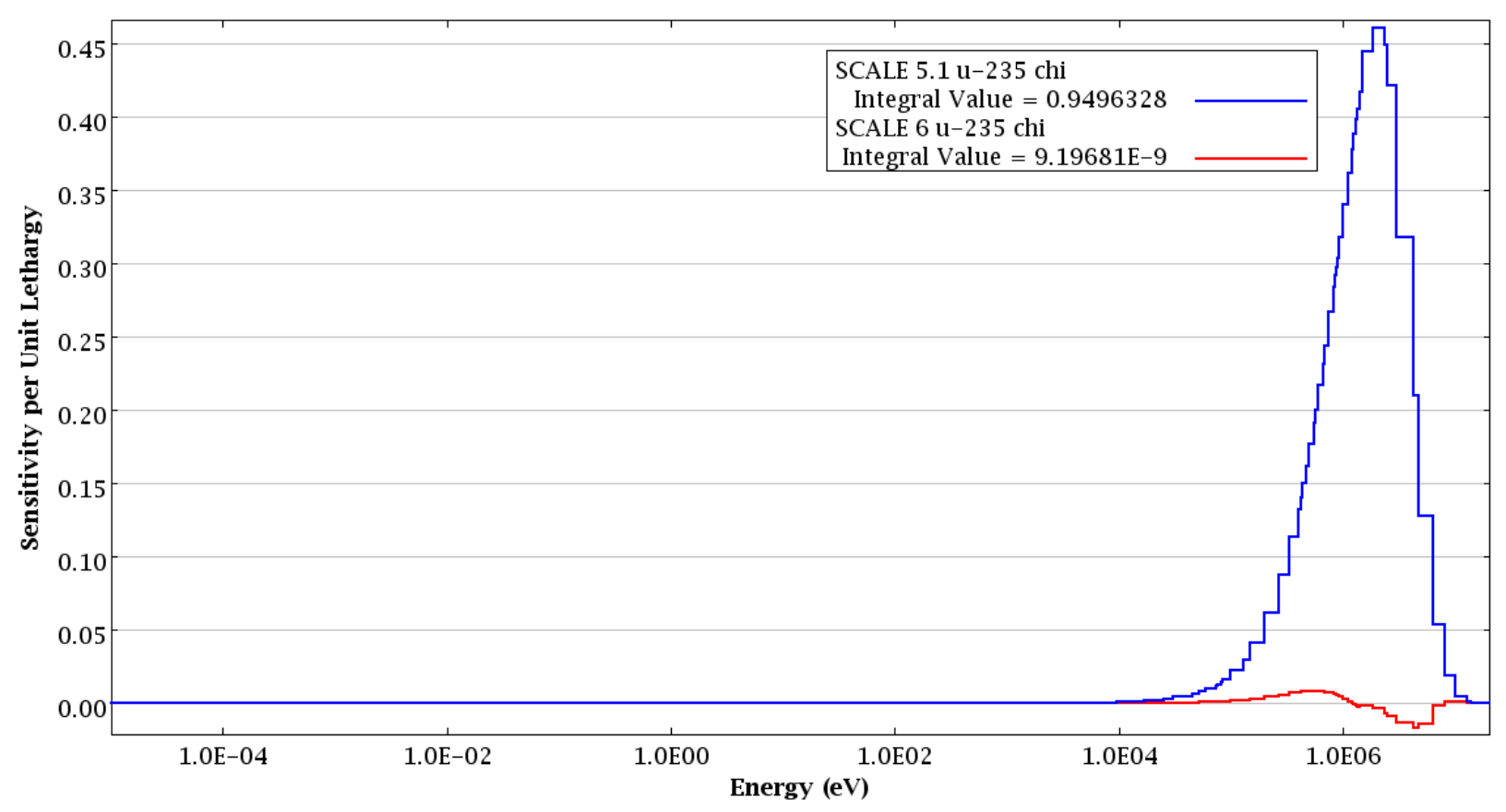

Fig. 31. Fission spectrum (chi) sensitivities for ${ }^{235} \mathrm{U}$ from SCALE 5.1 and SCALE 6. 


\section{TSUNAMI-3D EXAMPLE}

Now that you have the experience of running a TSUNAMI-1D example and viewing the output data, we can move on to a more complex case: a fuel assembly. This is a good example of what can be run as a $3 \mathrm{D}$ case in TSUNAMI-3D. The example will be very similar to the "Lattice Cell Example" from Sect. 7.5.1 of the KENO V.a Primer, ${ }^{8}$ with the exception of the new enrichment $\left(10 \%{ }^{235} \mathrm{U}\right)$, a few changes to the Parameters data, and the addition of SAMS data. For a more detailed description of how to input the data in GeeWiz, you can visit the KENO V.A Primer 8 The example begins on page 125 of that document.

Again, we will use GeeWiz as our means of creating the input file. Note that this example is also shown using SCALE 5.1, but the same general techniques are applicable in SCALE 6. The title of this example will be example 3D. In the Application menu, select TSUNAMI3D - tsunami-3d-k5. We will use the same cross-section processor and cross-section library we used in the previous example: NITAWLST and 238groupndf5, respectively. With these values entered, as shown in Fig. 32, click OK to close the General window.

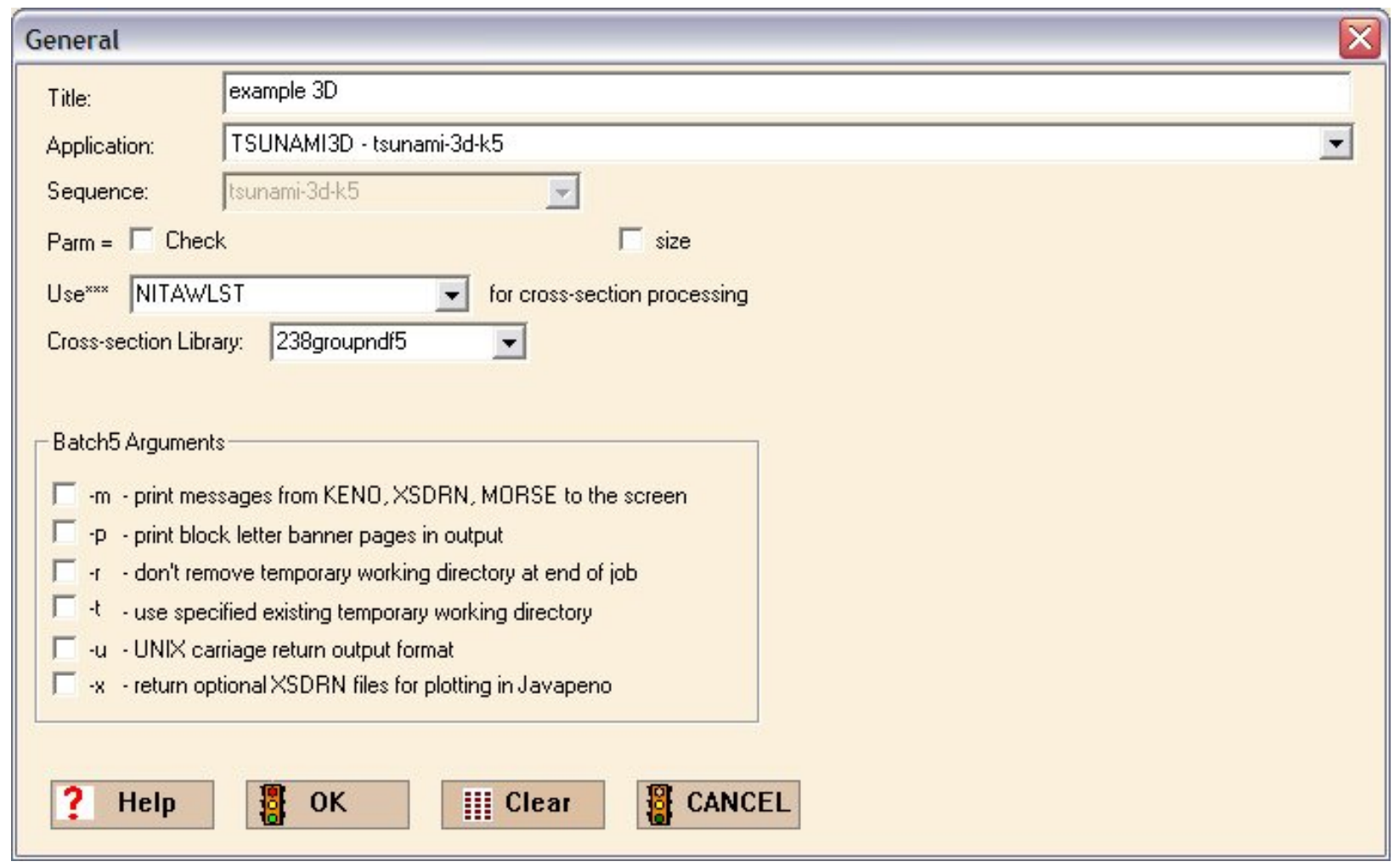

Fig. 32. General information for TSUNAMI-3D example.

Next, we enter the materials that will appear in our fuel assembly. We will use the fuel material from the previous example, $10 \%$ enriched $\mathrm{UO}_{2}$. The burnable poison rods are composed of $\mathrm{Al}_{2} \mathrm{O}_{3}-\mathrm{B}_{4} \mathrm{C}$, having a density of $3.7 \mathrm{~g} / \mathrm{cc}$, and are $1.260 \mathrm{wt} \% \mathrm{~B}_{4} \mathrm{C}$. The boron in the $\mathrm{B}_{4} \mathrm{C}$ is natural boron. The cladding is Zircaloy-2 (zirc2), a common material used in industry. The material for the shroud and end plugs in the rods is Type 304 stainless steel (SS304). Finally, the coolant and moderating material are water. To add 
these materials to the model, click the Compositions button on the left and then click the Create button. Click on Basic Compositions and enter in the following mixtures:

- mixture 1 as $10 \%$ uo2 with a density multiplier of 0.95 ,

- mixture 2 as $b 4 c$ with a density multiplier of 0.0126 (weight fraction of $\mathrm{B}_{4} \mathrm{C}$ in poison),

- $\quad$ mixture 3 as zirc2,

- $\quad$ mixture 4 as ss304, and

- mixture 5 as $h 2 o$.

When you have entered these mixtures, click OK. The $\mathrm{Al}_{2} \mathrm{O}_{3}$ that occurs in mixture 2 must be created manually because it is not in the SCALE Standard Composition Library. Click the Create button, but this time select the Compound (atom) option. Once the window opens, enter a 2 next to Mixture and enter a composition name of pois1 (which will appear as atompois1 in the input file). Input the density of $\mathrm{Al}_{2} \mathrm{O}_{3}$ as $3.74(\mathrm{~g} / \mathrm{cc})$. We will keep the default temperature but change the density multiplier to 0.9874 ( $1-0.0126)$, which is the weight fraction of $\mathrm{Al}_{2} \mathrm{O}_{3}$ in the poison. Then click on Insert Nuclide to begin building the compound. A new window should open and allow you to select a Nuclide to Insert. Select al from the scroll-down menu and then enter the Atoms per Molecule as 2. Click OK and repeat for 0 with a value of 3 for the Atoms per Molecule. Click OK twice. The compositions should now appear as shown in Fig. 33 .

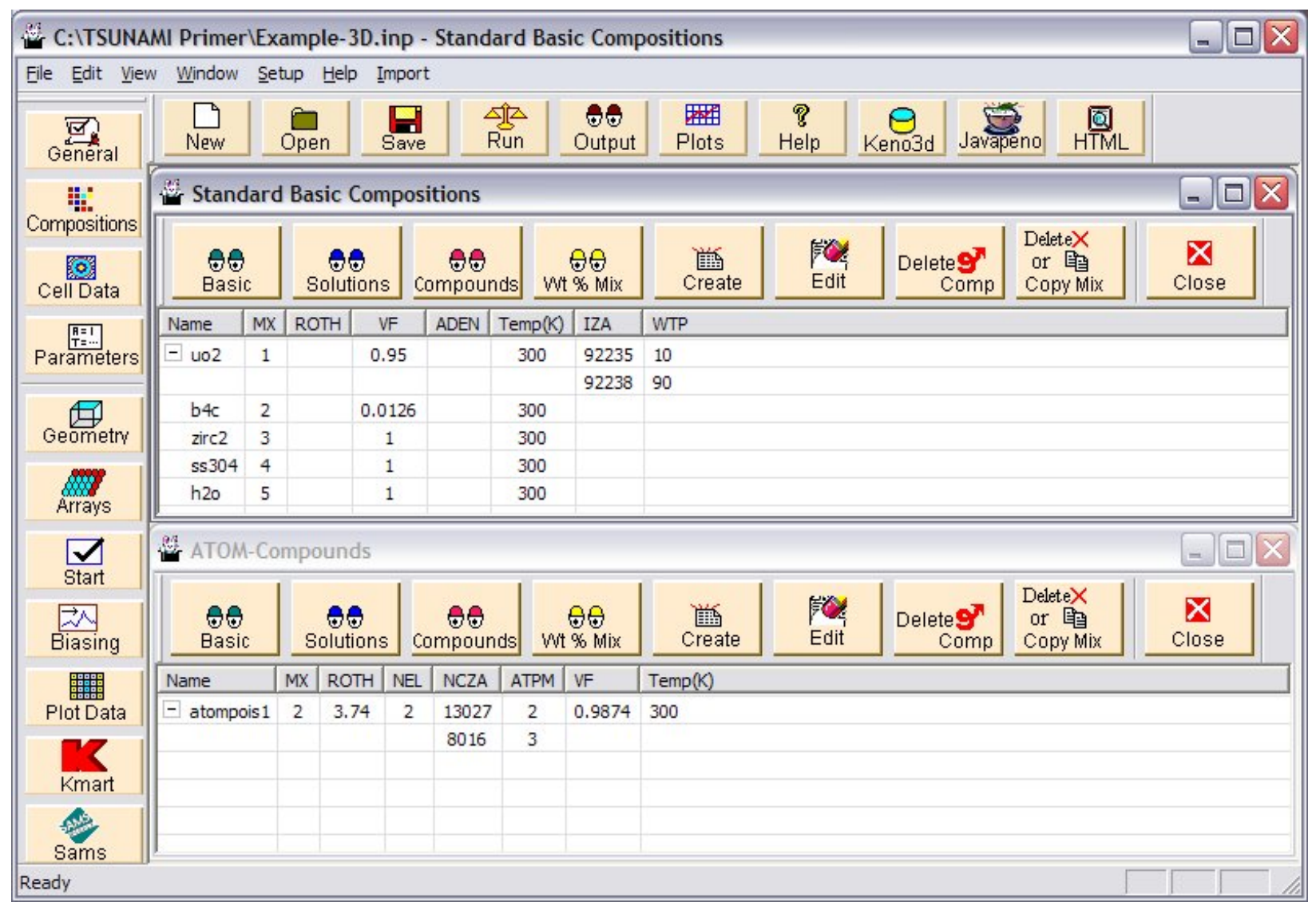

Fig. 33. Composition data for TSUNAMI-3D example. 
Next, click on the Parameters button. There are many options for a TSUNAMI-3D calculation, most of which are the same options available for KENO criticality calculations. We will set several important parameters to enable the accurate calculation of sensitivity coefficients. For sensitivity calculations, it is very important that both the forward and adjoint $k_{\text {eff }}$ calculations and the fluxes computed from those calculations be well converged. To accomplish this, we will request a large number of histories per generation and a large number of generations. We will take advantage of KENO's ability to stop the calculation when it has converged $k_{\text {eff }}$ to within a suitably small standard deviation. On the first tab, Key Parameters, set the number of generations for the forward calculation, GEN, to 10,000 . Also set the number of histories per generation for the forward calculation, NPG, to 5,000. Also check the boxes next to FDN to display fission densities and FLX to print fluxes, as shown in Fig. 34. Note that for TSUNAMI-3D, the number of generations skipped before accumulating $k_{\text {eff }}$ and flux data, NSK, is 50 . This is an increase from the default of 3 for NSK when running a standard criticality calculation with KENO. The reason for this increase is that we do not flux data from nonconverged generations to contaminate the final solution because the fluxes are the basis of the sensitivity calculations.

For this example problem, it will be sufficient to stop the forward calculation when $k_{\text {eff }}$ has converged to a standard deviation of $0.1 \%$, so in the second tab, Numeric Parameters, set SIG $=0.001$, as shown in Fig. 35. For production-level calculations, it may be necessary to converge the solution to $0.01 \%$ to reduce uncertainties in the sensitivity coefficients.

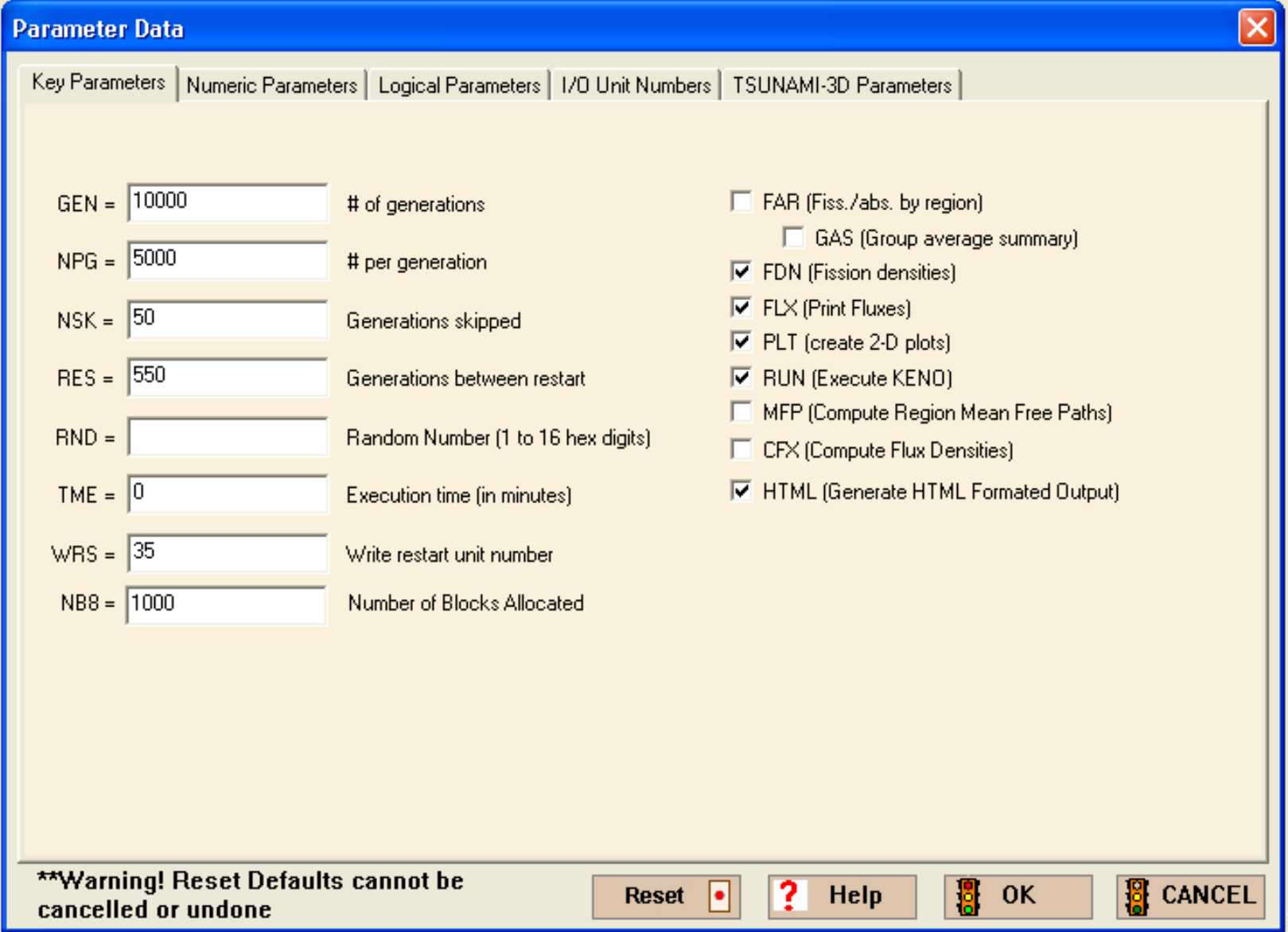

Fig. 34. Key parameters form for TSUNAMI-3D example. 


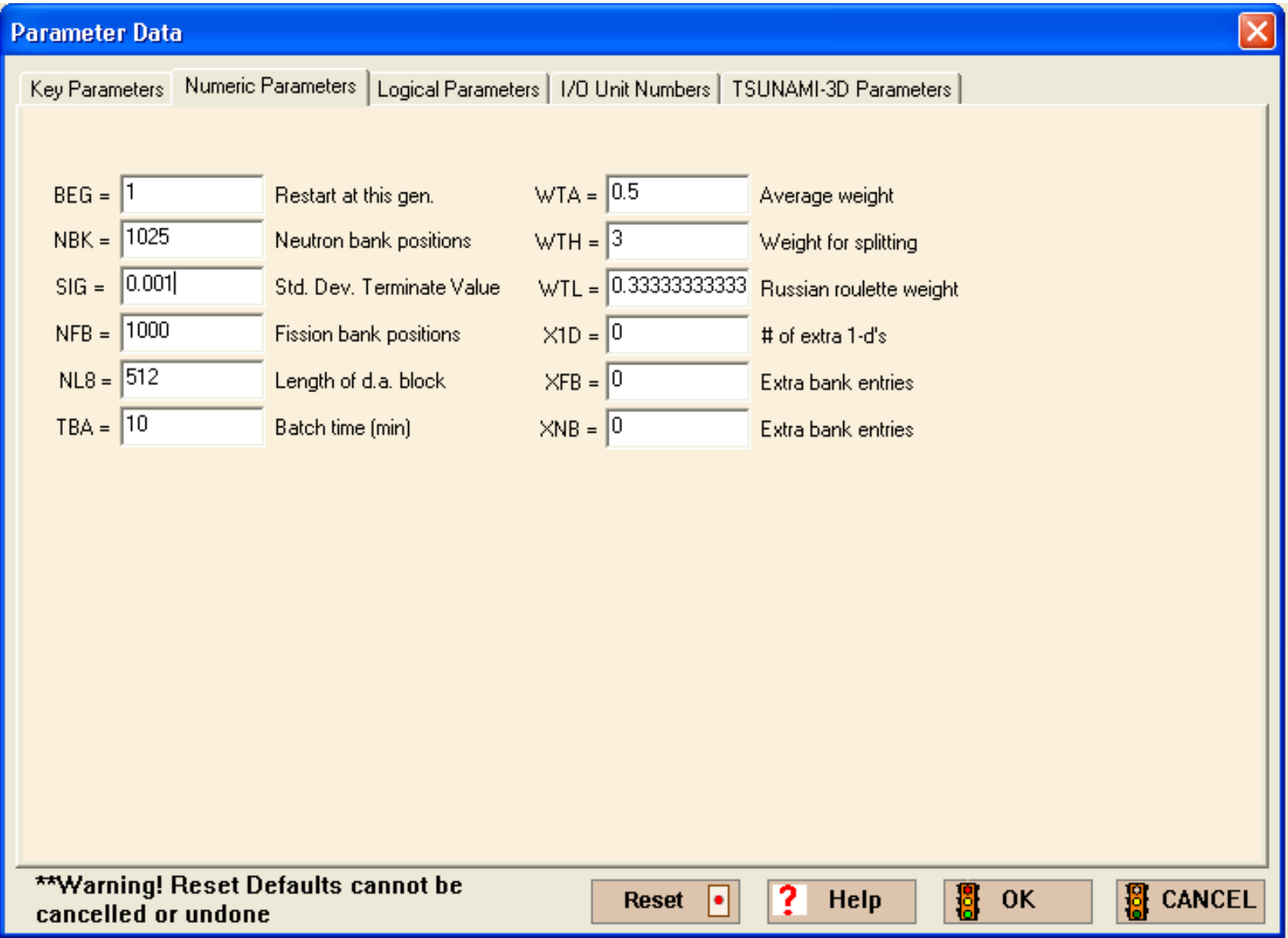

Fig. 35. Numeric parameters for TSUNAMI-3D example.

Now we need to set parameters that were developed specifically for TSUNAMI-3D calculations in the last tab, TSUNAMI - 3D Parameters. Here you can control the adjoint calculation separately from the forward calculation; control the calculation of angular fluxes and angular flux moments, which are especially important for the sensitivity of $k_{\text {eff }}$ to scattering cross sections; and add a spatial mesh for flux accumulation, which is important for capturing differences in the flux characteristics across the system model. Note that several TSUNAMI-3D parameters are automatically set based on the values selected for the forward calculation. The number of particles per generation for the adjoint calculation, APG, is automatically set to three times the value set for the forward calculation. This is required because adjoint solutions require more sampling than forward solutions, especially for intermediate and fast spectrum systems. Also, adjoint solutions converge much more slowly than forward solutions, so the number of generations skipped is defaulted to three times the number skipped for the forward calculation. The convergence criterion to complete the adjoint calculation, $A S G$, is set to the same value as the forward calculation.

We will now make some adjustments to the default values to quickly provide reliable results that demonstrate TSUNAMI-3D. To accelerate this calculation, we will request only the angular moments of the flux solution computed to first order by setting PNM $=1$. In general, it is recommended that PNM remain at its default value of 3 to better capture the sensitivity of $k_{\text {eff }}$ to anisotropic scattering, but a first-order solution will run about twice as fast and will provide reasonable results for this system. We will also disable the coordinate transform by setting TFM to no. Disabling the coordinate transform will 
speed up the calculation by about one-third and provide accurate results if a spatial mesh is used. Next, we will introduce a spatial mesh to accumulate the fluxes by setting MFX to yes and entering a mesh interval size of $5 \mathrm{~cm}$ by setting $\mathrm{MSH}=5$. A general recommendation for selecting the interval size for the spatial mesh is to use one-tenth the overall fuel dimension. For this fuel-bundle model, selecting onetenth the bundle width would require a mesh size of about $1.4 \mathrm{~cm}$, which would result in a large number of intervals along the length of the fuel bundle. TSUNAMI-3D has no limit to how many mesh intervals can be used, but groupwise fluxes and their angular moments must be computed and stored for each mesh interval. A problem with millions of mesh intervals is that 238-group fluxes and third-order moments can require many gigabytes of RAM and result in very long run times. With the 5-cm mesh interval set here, this problem will run with about $200 \mathrm{MB}$ of RAM. The values selected for PNM, TFM, MFX and MSH are applied to both the forward and adjoint calculations.

Next, we will make some adjustments to the default parameters related to the adjoint calculation. To better sample each generation, we will set $A P G=30,000$. Also, to shorten the overall run time, we will stop the adjoint calculation when it has converged to $0.5 \%$ in $k_{\text {eff }}$ by setting $A S G=0.005$. In production-level calculations, the adjoint solution should be allowed to converge to a lower standard deviation, such as $0.05 \%$. However, obtaining this level of convergence requires hours of run time. The final TSUNAMI-3D parameters are shown in Fig. 36.

Key Parameters | Numeric Parameters | Logical Parameters | I/0 Unit Numbers TSUNAMI-3D Parameters |

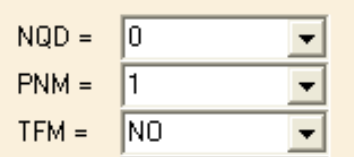

$\mathrm{APG}=30000$
$\mathrm{ABK}=30000$

$A G N=\longdiv { 1 0 1 0 0 }$

$\mathrm{PMF}=\mathrm{NO} \quad \boldsymbol{V}$

$M F X=Y E S \quad-\quad$

$\mathrm{MSH}=5$

$\mathrm{PMS}=\mathrm{NO}$

$\mathrm{PMV}=\mathrm{NO} \quad \mathrm{V}$

$\mathrm{PMM}=\mathrm{NO} \quad \mathbf{F}$

$A S K=\longdiv { 1 5 0 }$

$A S G=\longdiv { 0 . 0 0 5 }$ order of angular quadrature

order of flux moment

compute angular fluxes or moments with transformed directions

number of neutrons per generation for adjoint calculation

number of neutron bank positions for adjoint case

number of generations for adjoint case

print more fluxes

compute mesh fluxes

Size of mesh

print mesh fluxes

print mesh volumes

print mesh flux moments if calculated

number of generations skipped for adjoint calculation (default is $3^{\mathrm{x}}$ NSK key parameter)

convergence crteria on $\mathrm{k}$-eff for adjoint (default is numeric parameter SIG]

Fig. 36. TSUNAMI-3D parameters for TSUNAMI-3D example. 
Next, we will enter cell data for resonance self-shielding of the cross sections. Because we are creating a fuel assembly that is a repeating lattice of fuel rods, click on the Lattice Cell tab on the Cell Data form, then select new cell. Select SquarePitch from the pull-down menu for Type of Lattice. The half pitch of the unit cell is $0.82 \mathrm{~cm}$, and the fuel radius is $0.47 \mathrm{~cm}$. Select the fuel mixture $1 \mathrm{uo} 2$ and the moderator mixture $5 \mathrm{~h} 20$ from the pull-down menus. This case has clad and gap, so check the box Clad/Gap Exists, which will display additional input fields. Enter a Clad Radius of $0.545 \mathrm{~cm}$ and select the Clad Mixture as 3 zirc2. The Gap Radius is $0.4875 \mathrm{~cm}$, and the Gap Mixture is $\odot$ void. The completed Lattice Cell Data form is shown in Fig. 37.

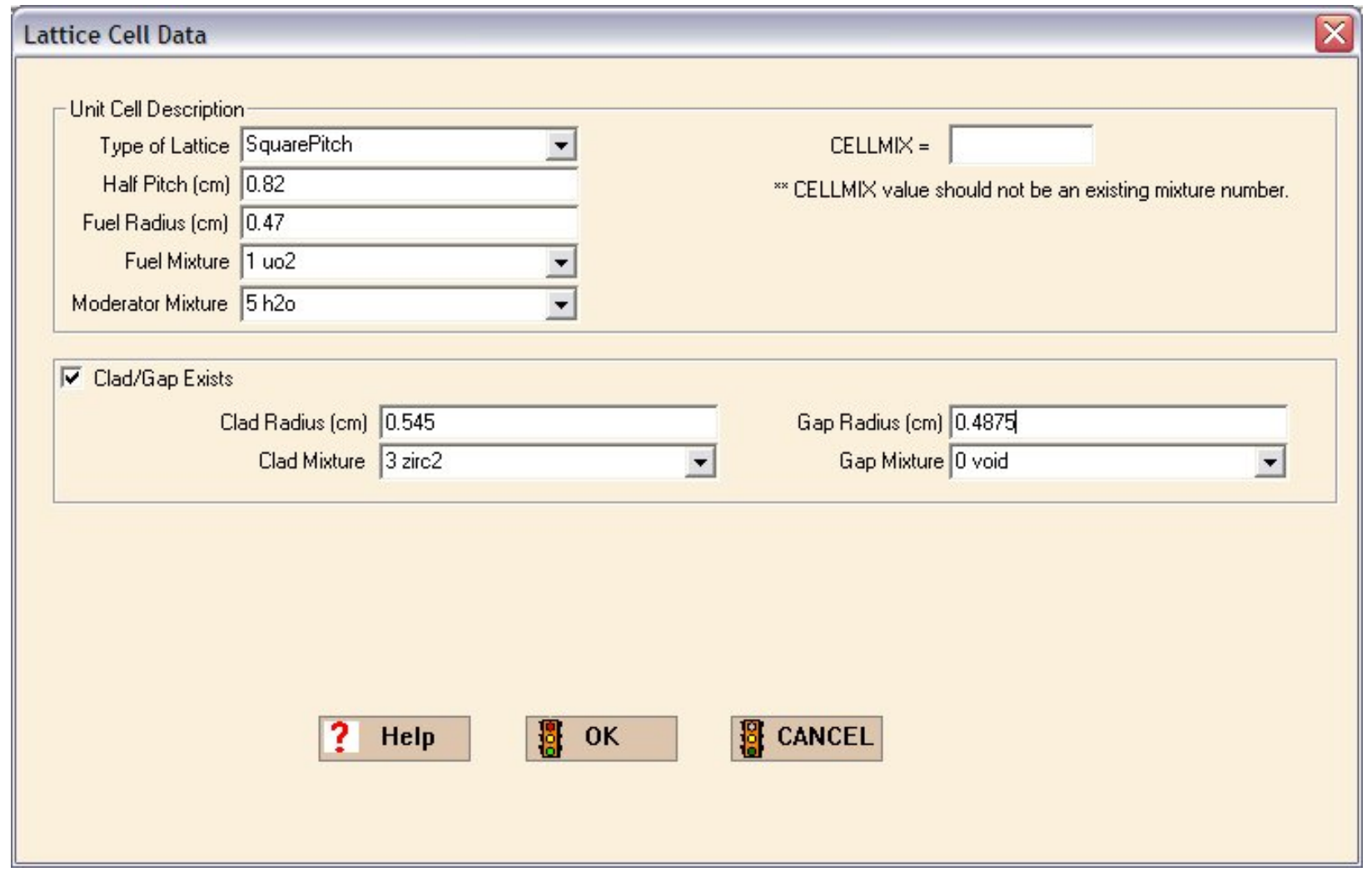

Fig. 37. Lattice cell data form for TSUNAMI-3D example.

Next, we will enter the geometry specifications. The basic units are a fuel rod, a burnable poison rod, a water hole, and the global unit that contains an array of all three types of rods as well as additional coolant material and a shroud. We begin by creating the first three units. Click on the Geometry button in the left toolbar to begin a new unit. Enter the Comment for Unit 1 as $10 \%$ uo2 rods. The process of building the rod starts with the innermost region and moves outward. To begin, click on the Cylinder button on the right. Select the $1 \mathrm{uo} 2$ as the mixture for the fuel and input the dimensions of the fuel rod-Radius $=0.47 \mathrm{~cm}, Z \max =182.88 \mathrm{~cm}, \mathrm{Zmin}=-182.88 \mathrm{~cm}$ - then press OK. The next region is the gap, which is assumed to be void. Press the Cylinder button again, select $\odot$ void from the Mixture menu, and input the dimensions-Radius $=0.4875 \mathrm{~cm}, \mathrm{Zmax}=199.84 \mathrm{~cm}, \mathrm{Zmin}=-206.24$ $\mathrm{cm}$ - then press OK. For the clad, click on Cylinder, select 3 zirc2 in the Mixture menu, and input the data-Radius $=0.545 \mathrm{~cm}, Z \max =199.84 \mathrm{~cm}, \mathrm{Zmin}=-206.24 \mathrm{~cm}$-then press OK. Now, we must add the SS304 end caps. Again, press the Cylinder button and select 4 ss304 from the 
Mixture menu. We will enter the same Radius $(0.545 \mathrm{~cm})$ but Zmax $=210.34 \mathrm{~cm}$ and $\mathrm{Zmin}=$ $-210.34 \mathrm{~cm}$. These data add cylinders of SS304 with the same radius as the clad above and below the clad. Press OK when you have entered these data. The fuel-rod cell also contains water surrounding the fuel rod in a cuboid with dimensions of the fuel rod pitch. To add this, select Cuboid on the right toolbar, select $5 \mathrm{~h} 20$ from the Mixture menu, and enter the positive and negative values of the half pitch for $+\mathrm{X},-\mathrm{X},+\mathrm{Y}$, and $-\mathrm{Y}( \pm 0.82 \mathrm{~cm}$, respectively). Enter the same values for $+\mathrm{Z}$ and $-\mathrm{Z}$ as the fuel rod $( \pm 210.34 \mathrm{~cm}$, respectively), then press OK. This completes a unit that contains a fuel rod and its surrounding water coolant/moderator, as shown in Fig. 38.

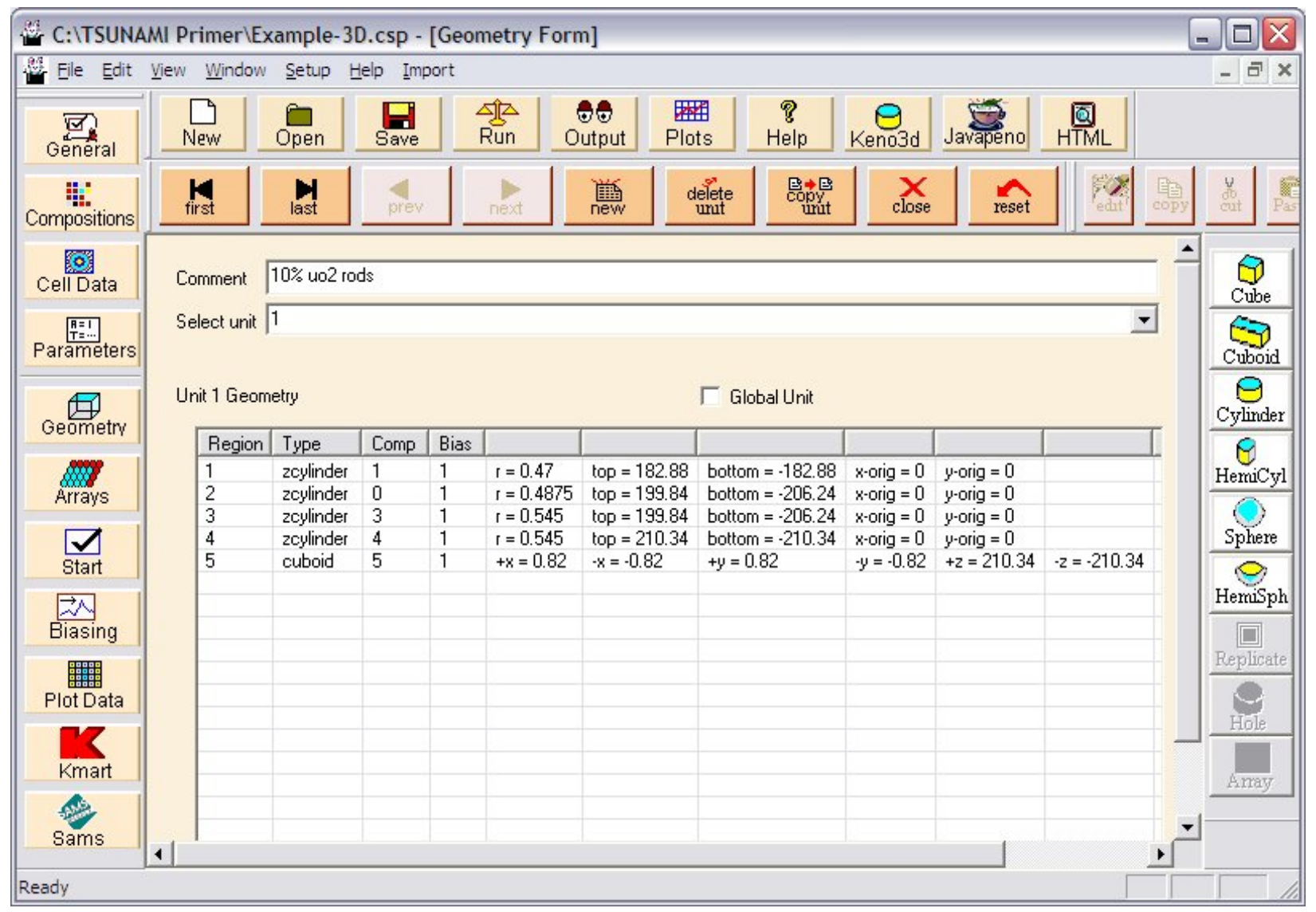

Fig. 38. Unit 1 fuel rod geometry data.

Click new to create a new unit and repeat similar steps for Unit 2, titled burnable poison rod. For the burnable poison mixture $(2 \mathrm{~b} 4 \mathrm{c}$, atompoison1), the Radius $=0.545 \mathrm{~cm}, Z \max =160 \mathrm{~cm}$, and $Z \min =-160 \mathrm{~cm}$. The void Radius $=0.545 \mathrm{~cm}$, as there is no gap, with $Z \max =260.48 \mathrm{~cm}$ and $\mathrm{Zmin}=-$ $160 \mathrm{~cm}$. The clad Radius $=0.645 \mathrm{~cm}$, while $Z \max =260.58 \mathrm{~cm}$ and $Z \min =-160.1 \mathrm{~cm}$. This unit includes the surrounding water with dimensions of the half pitch $( \pm 0.82 \mathrm{~cm})$ for $\mathrm{X}$ and $\mathrm{Y}$ and the same $\mathrm{Z}$ dimensions as the clad for Unit 2.

Next, create Unit 3, titled water hole (instrumentation), as the instrumentation tube, which we will assume to be water only. This unit contains only one region, a cuboid of water with the dimensions of the water cuboid in Unit 2. 
Before we create the Global Unit, which is the fuel assembly unit, we need to create an array to define the lattice arrangement of the fuel assembly. To do this, click the Arrays button on the left side of the screen and enter the comment fuel assembly. Click the Create button that appears on the top toolbar of the Arrays window. The array is designated Array 1 (Number $=1)$. Our case is a $9 \times 9$ fuel assembly, so enter NUX $=9$ and NUY $=9$. It is easiest to initialize the array with Unit 1 (fuel rods) because that will require the fewest changes later. Click $O K$ to create the array. With the $9 \times 9$ array containing all fuel rod units (Unit 1), we must change eight to burnable poison rod units (Unit 2) and one to the water hole instrumentation unit (Unit 3). This can be done by clicking on the hand pointer tool on the top of the right toolbar. Once it is enabled, click on the red box next to Unit 2 and then click on the locations of the burnable poison rods, shown in Fig. 39. Next, place the water hole in the center of the fuel assembly by clicking on the green box next to Unit 3 and then clicking on the location of the central instrumentation tube. The pattern should look like that shown in Fig. 39. When you have completed this pattern, click Close on the array toolbar.

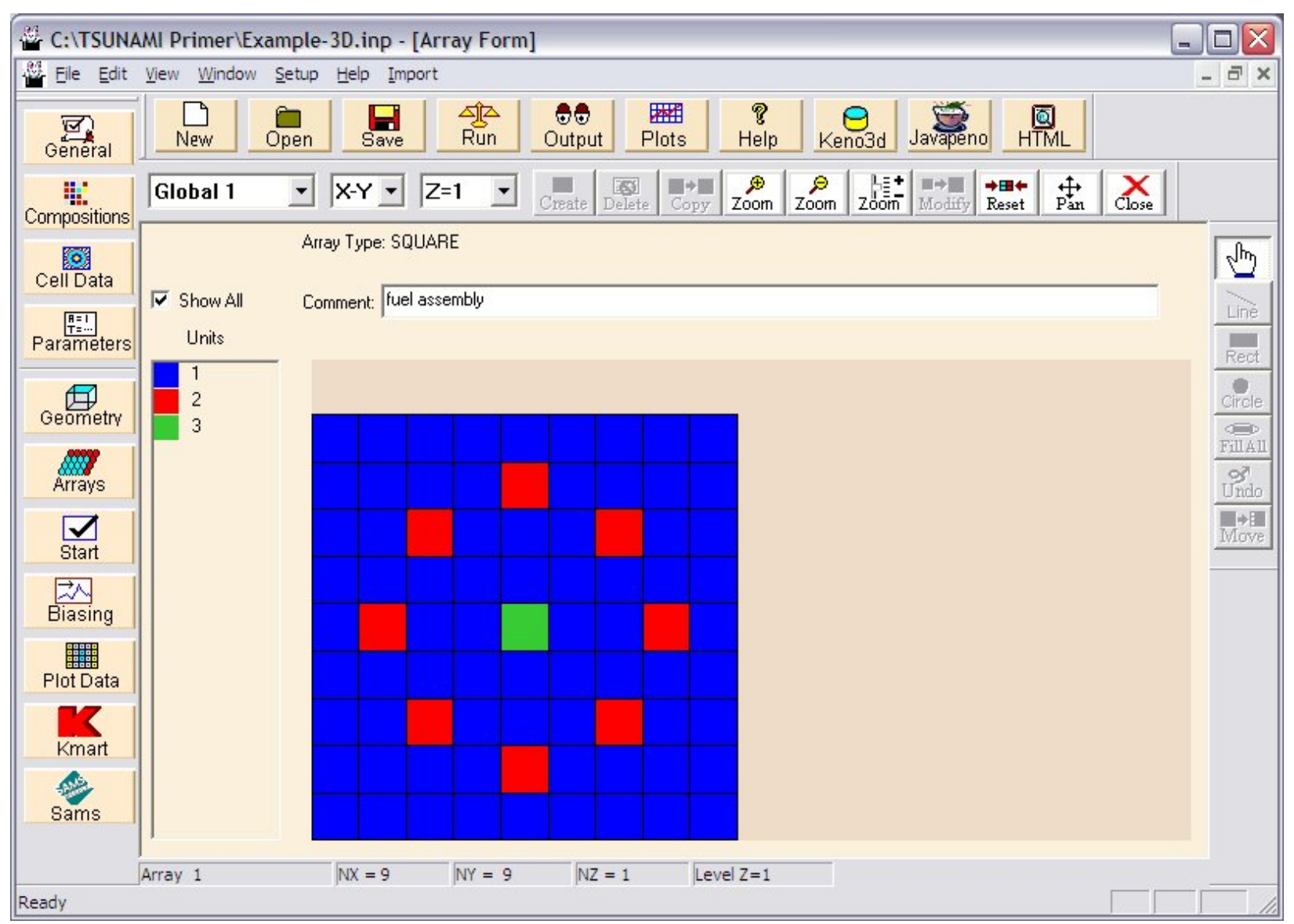

Fig. 39. Array 1 form for TSUNAMI-3D example.

Return to the Geometry Form to complete the global unit. Add a new Unit 4 and name it fuel assembly. Click on the Array button on the right toolbar of the Geometry Form to add the array to this unit. Select Array 1 from the pull-down menu (it should be the only array available) and then enter the array placement coordinates of $X$-neg $=-7.38 \mathrm{~cm}, Y$-neg $=-7.38 \mathrm{~cm}, Z$-neg $=-210.34 \mathrm{~cm}$ to move the origin of the global unit to the center of the array $(9 \times$ half pitch $=7.38 \mathrm{~cm}$, and $210.34 \mathrm{~cm}$ is half the assembly 
height). Add a cuboid of water with the dimensions $\pm X= \pm Y= \pm 7.48 \mathrm{~cm}, \pm Z= \pm 210.34 \mathrm{~cm}$, which defines the water channel surrounding the assembly. A SS304 shroud is added around the bundle using the Replicate option on the right toolbar. First, select the row in the geometry table that you want to replicate, in this case it is Region 2, then press the Replicate button. Select 4 ss304 from the Mixture pull-down menu. The thickness of the shroud is $0.16 \mathrm{~cm}$, so input this value for all $\mathrm{X}, \mathrm{Y}$, and $\mathrm{Z}$ fields, and press OK. Finally, a water reflector is added around the assembly. This time you will replicate Region 3 with a water reflector of thickness $\pm X= \pm Y=7.62 \mathrm{~cm}, \pm Z=15.24 \mathrm{~cm}$. Click on the Global Unit checkbox to indentify this unit as the outermost unit in the geometry.

With the data for Unit 4 completed as shown in Fig. 40, click Close on the top toolbar on the Geometry Form.

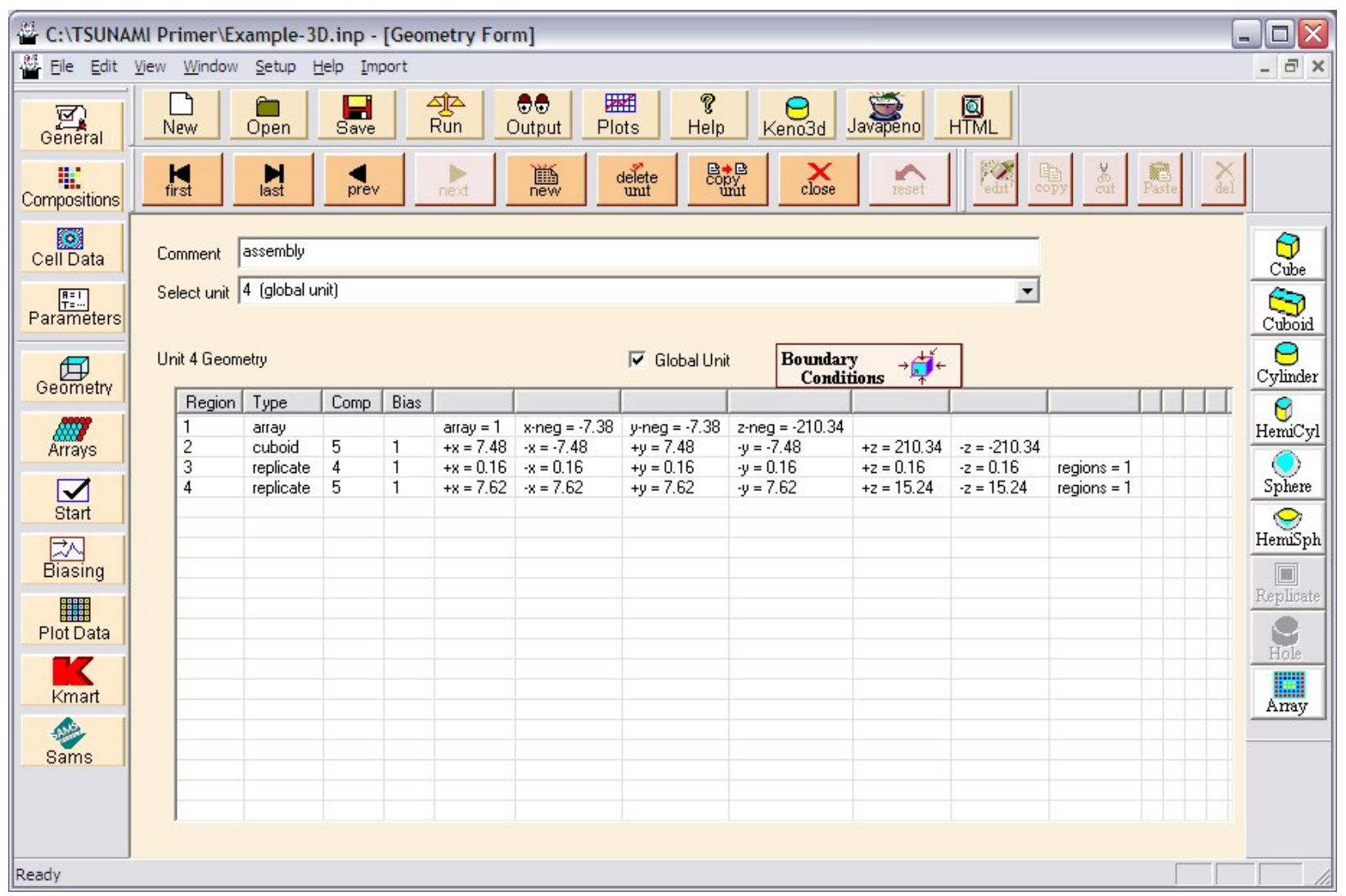

Fig. 40. Global unit geometry data for TSUNAMI-3D example problem.

There are additional options on the left toolbar associated with KENO for TSUNAMI-3D: Start, Biasing, Plot Data, and Kmart. These are the same options available for standard criticality calculations and will not be discussed here. However, we still need to make the changes to the SAMS parameters that we did in the TSUNAM-1D example. Click the SAMS button and change the covariance library to 44groupv5rec, the recommended covariance data for ENDF/B-V calculations. Next, click the checkboxes next to prtgeom and prtimp to list sensitivities for each geometrical region and to edit the implicit sensitivity data, respectively. The SAMS data form should appear as shown in Fig. 41. Note that with SCALE 6 a new covariance library, 44groupcov, will be the only library available. 
Covariance data file (coverx $=$ ]

44groupv5rec

$\mathrm{pn}=\sqrt{3}$ Legendre order of moments calculation

largeimp $=\sqrt{100}$ cutoff value for group-wise implicit sensitivities

$\Gamma$ binsen - produce SENPRO data

『 makeimp - make implicit sensitifity coefficients

$\Gamma$ nocovar - turn off uncertainty edit

$\Gamma$ nomix - turn off sensitivities by mixture

$\Gamma$ prtflx - print angular fluxes and flux moments

$\sqrt{V}$ prtgeom - output sensitivities for each geometry region

ॠ prtimp - print implicit sensitivity coefficients

$\Gamma$ prtvols - SAMS print volumes of regions

$\Gamma$ nohtml - Supress html formatted output from SAMS

$\Gamma$ pmeshvol - print mesh volumes

Calculation option:

(- usemom - use flux moments

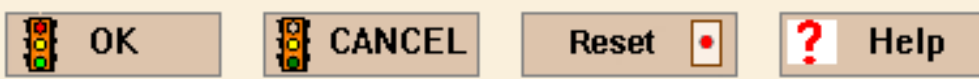

Fig. 41. SAMS data form for TSUNAMI-3D example problem.

The input for TSUNAMI-3D is now complete. Save the input file as Example-3D.inp, then press the Run button to execute SCALE. This calculation will run in about $29 \mathrm{~min}$ on a $2.5 \mathrm{GHz}$ Intel Core2 Duo processor. The forward KENO calculation requires less than $5 \mathrm{~min}$, and the adjoint requires about 22 $\min$.

Open the output by clicking the HTML button in GeeWiz. Because KENO V.a also creates HTML output, there are three separate output listing available. Below the title of your input file (in our case example 3d), you should see the options 1. KENO V.a, 2. KENO V.a, and 3. SAMS, as shown in Fig. 42. The first item displays the results for the KENO V.a forward case, the second shows the KENO V.a adjoint case, and the third displays the SAMS results. You can return to this page from anywhere in the HTML output by clicking on the SCALE icons at the top of the page. Clicking on the SCALE icons in this page will take you to the SCALE website at ORNL, if your computer is connected to the Internet. 


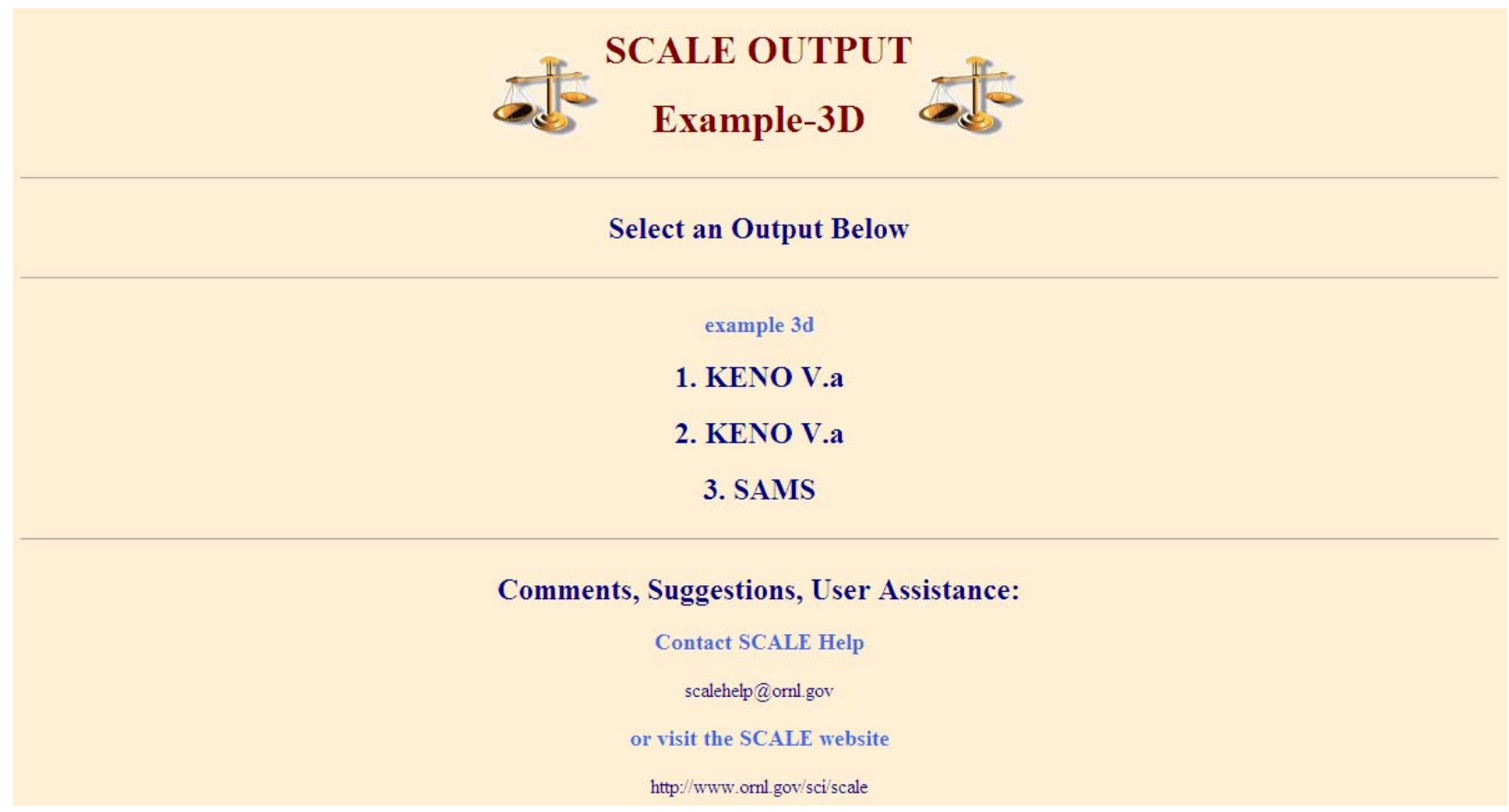

Fig. 42. Initial page of HTML output from TSUNAMI-3D example.

It is recommended that users review the forward and adjoint calculations before viewing the SAMS results to ensure that the calculations do not present any anomalies. Also, the $k_{\text {eff }}$ values for both the forward and adjoint calculations should be equivalent. Any mismatch in $k_{\text {eff }}$ outside the statistics likely indicates that the adjoint calculation was under sampled. The Final Results HTML page for the KENO V.a forward calculation is shown in Fig. 43, and the one for the adjoint calculation is shown in Fig. 44. The calculated $k_{\text {eff }}$ value for the forward case is $0.755 \pm 0.001$ and for the adjoint case is $0.755 \pm$ 0.005 , so the results agree well. As noted in the previous chapter on reviewing the output, review any error and warning messages to ensure that the cases ran correctly. 


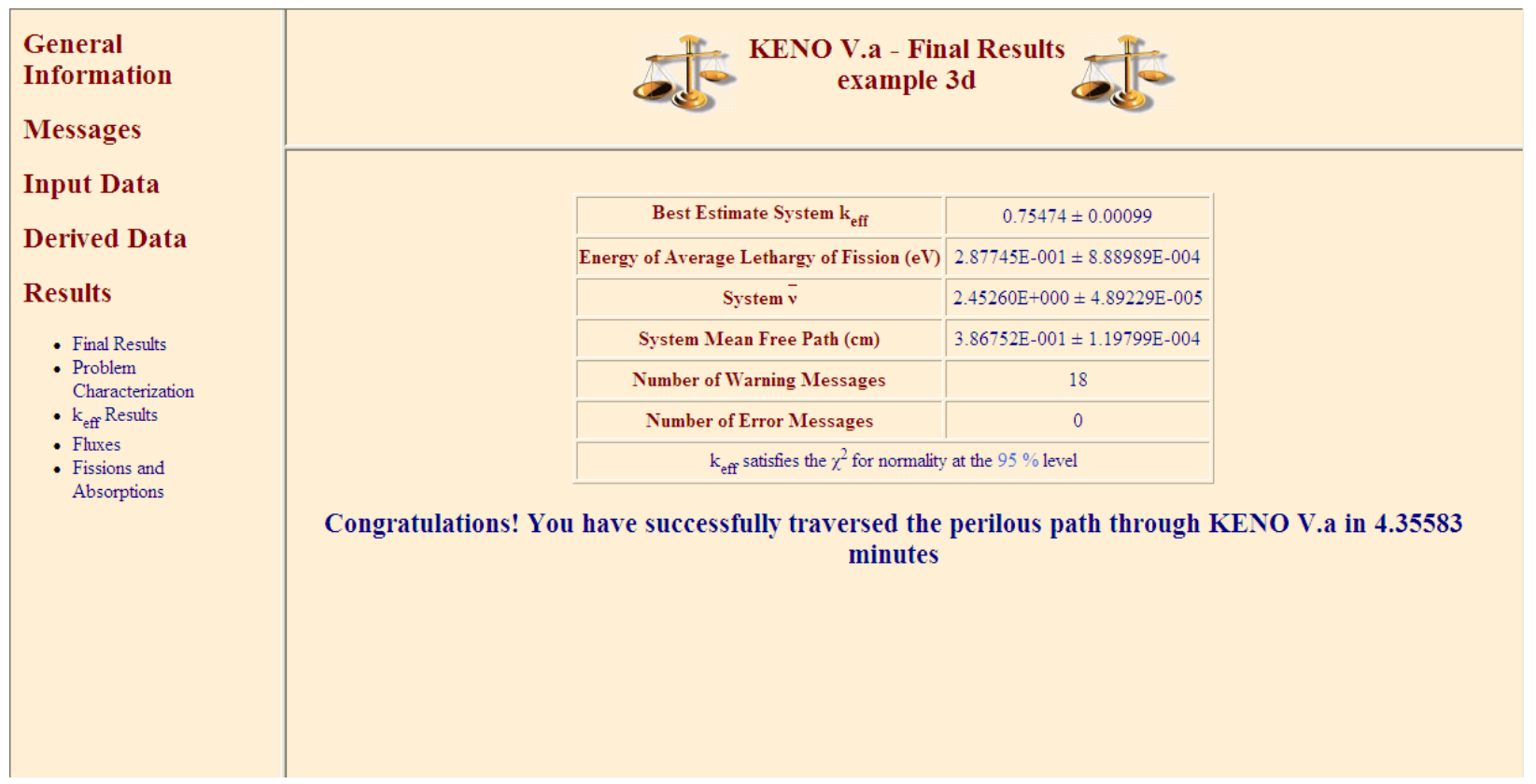

Fig. 43. KENO V.a forward calculation final results page for TSUNAMI-3D example.

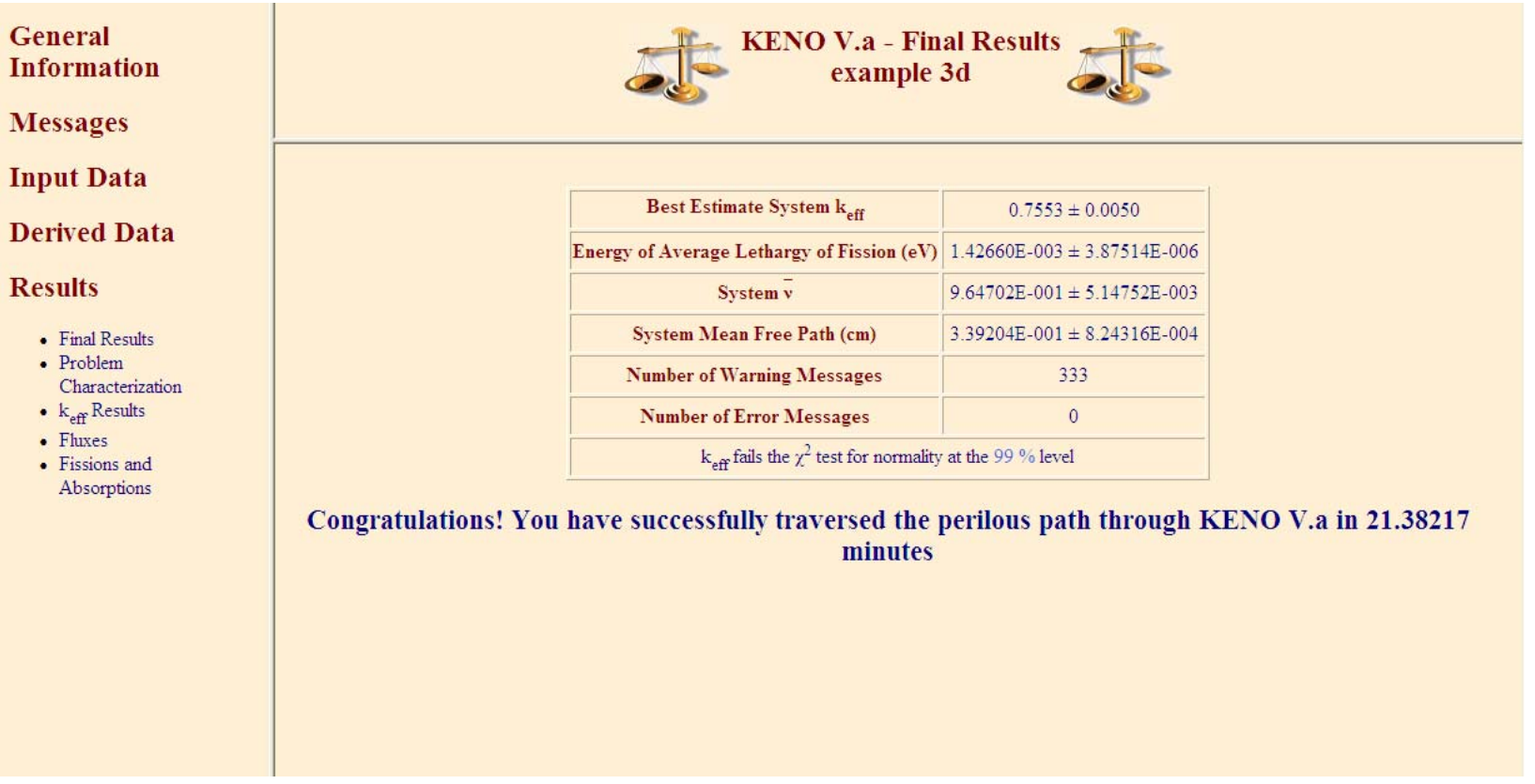

Fig. 44. KENO V.a adjoint calculation final results page for TSUNAMI-3D example.

The format of the SAMS output is very similar to that presented in Sect. 3. The Summary of Transport Calculations, shown in Fig. 45, includes general information regarding the forward and adjoint KENO calculations, including the $k_{\text {eff }}$ results. Also, SAMS generates a warning message if the forward and adjoint $k_{\text {eff }}$ values disagree by more than $1 \%$. 


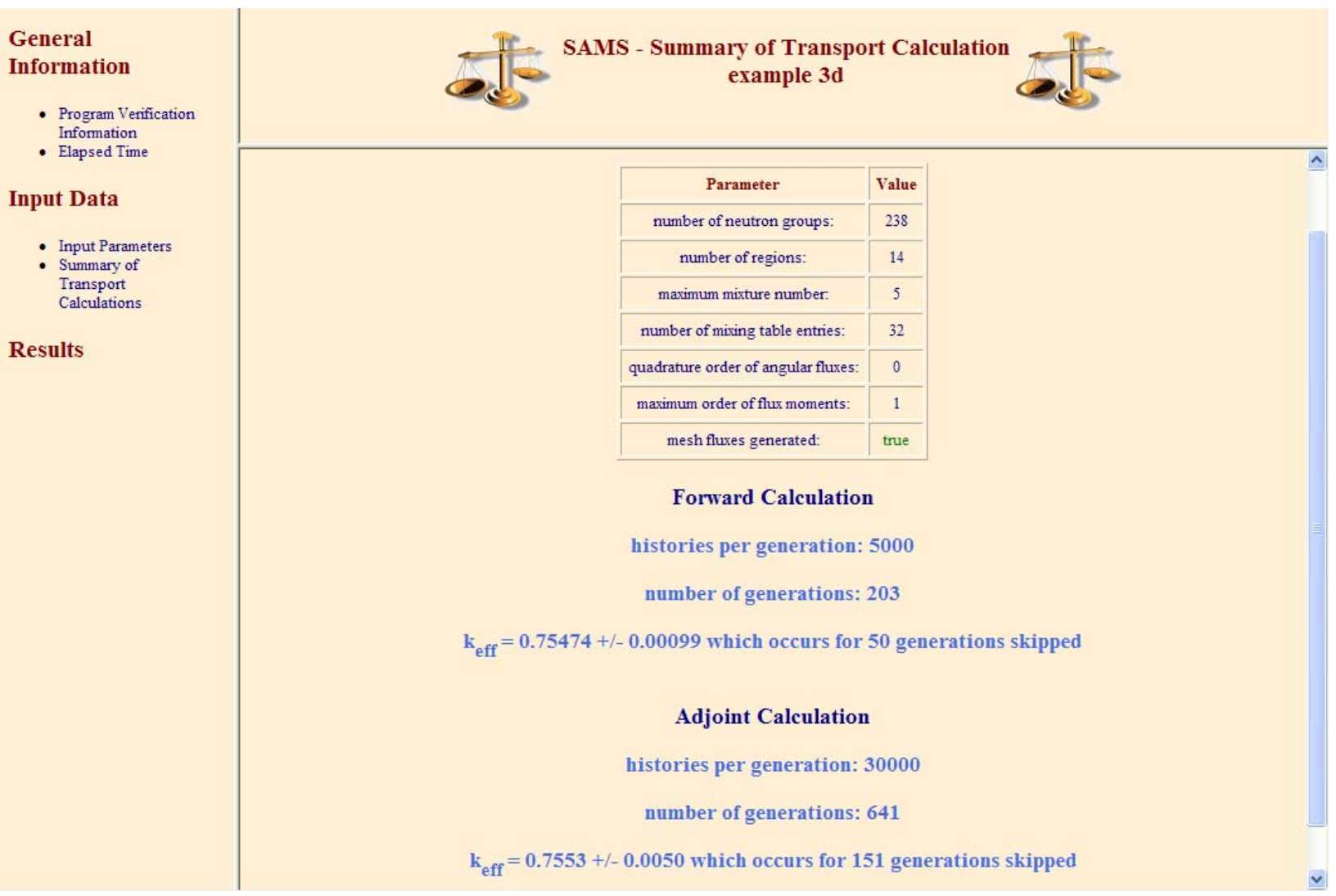

Fig. 45. Summary of transport calculation from TSUNAMI-3D example.

The data in the Results section are very similar to those previously discussed for the TSUNAMI-1D example, but the TSUNAMI-3D results also include statistical uncertainties resulting from the Monte Carlo calculation. The edit of Energy, Region and Mixture Integrated sensitivity coefficients is shown in Fig. 46. Note that each value, previously described for TSUNAMI-1D, now includes Monte Carlo uncertainties. 


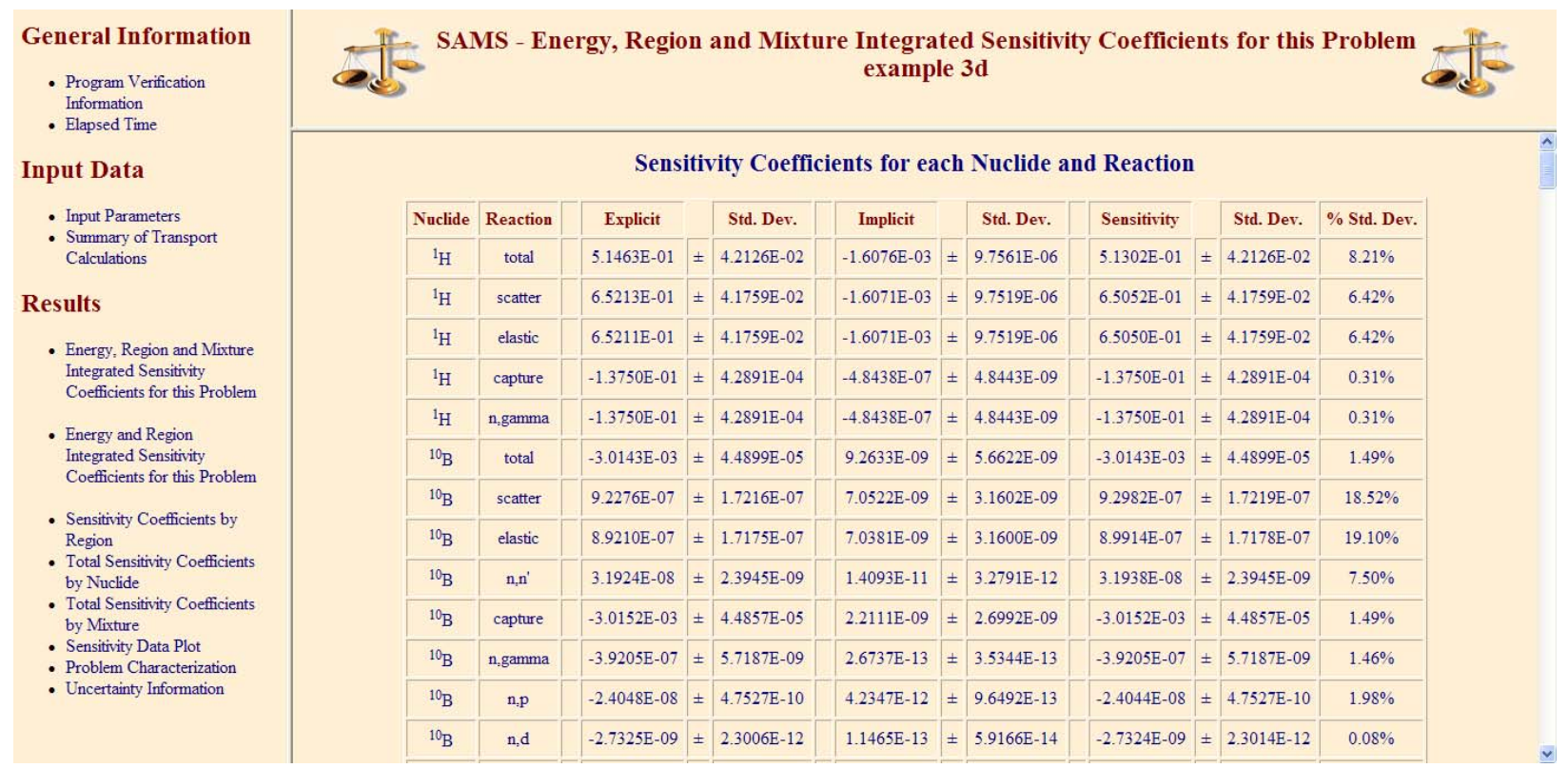

Fig. 46. Energy, region, and mixture integrated sensitivity coefficients from TSUNAMI-3D example.

The Sensitivity Coefficients by Region edit, shown in Fig. 47, contains similar information but is more complex than the Sensitivity Coefficients by Material Zone edit for TSUNAMI-1D. Because KENO has multiple units, each of which can contain multiple zones, an additional menu is presented on the right side of the output. The top section of this menu offers a convenient means of navigating the bottom section of the menu. Selecting a unit from the top menu will set the bottom menu to the specified unit. From the bottom menu, selecting a region will display the sensitivity data for that unit and region in the main display frame.

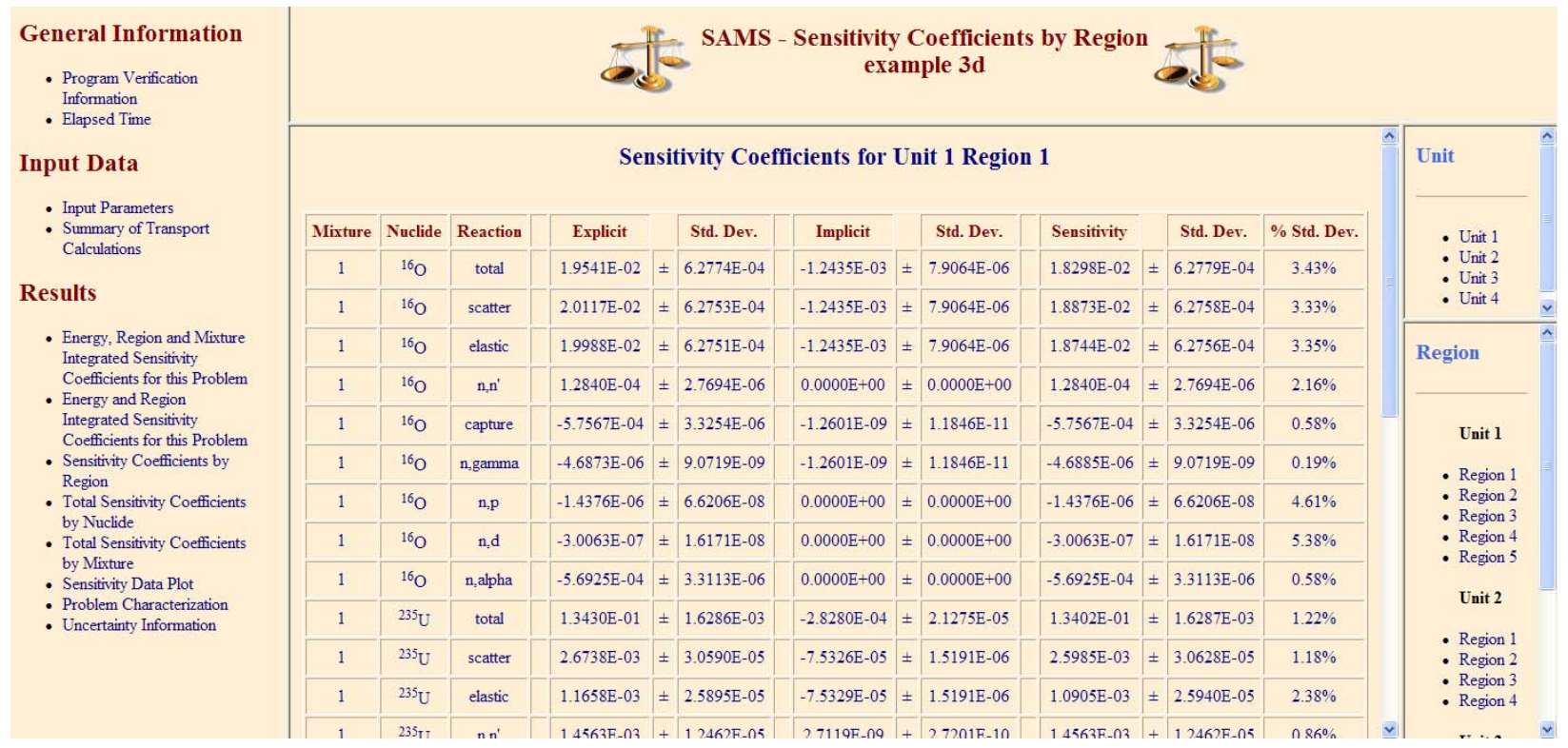

Fig. 47. Sensitivity coefficients by region from TSUNAMI-3D example. 
The Sensitivity Data Plot for TSUNAMI-3D is also very similar to that for TSUNAMI-1D, but the values shown in the plot also include error bars representing 1 standard deviation in the sensitivities resulting from the Monte Carlo calculation. An example plot is shown in Fig. 48.

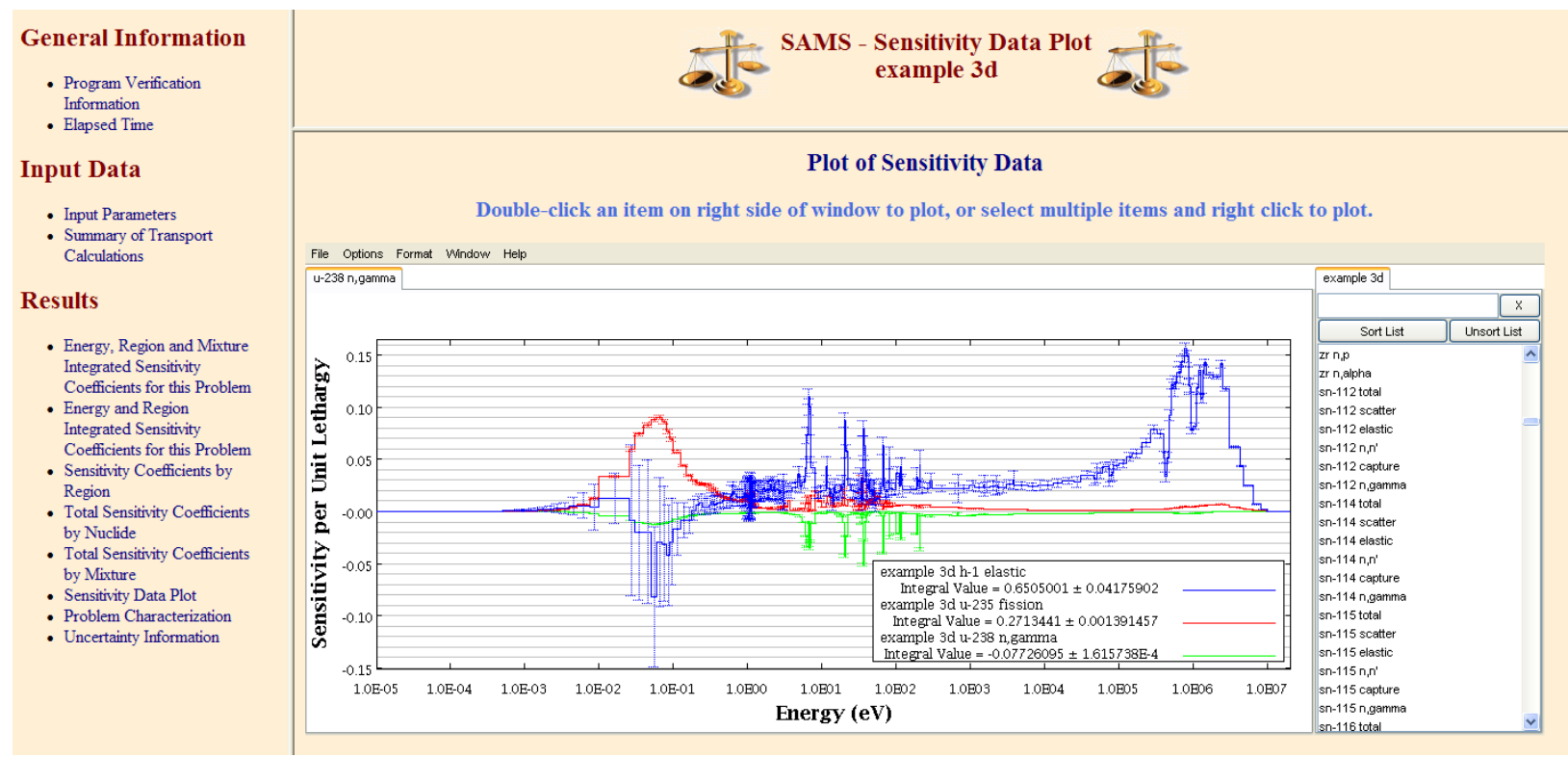

Fig. 48. Sensitivity data plot from TSUNAMI-3D example.

The Uncertainty Information edit from TSUNAMI-3D is also very similar to that described for TSUNAMI-1D, but it also includes Monte Carlo uncertainties propagated to provide a statistical uncertainty on the uncertainties in $k_{\text {eff }}$ due to cross-section-covariance data.

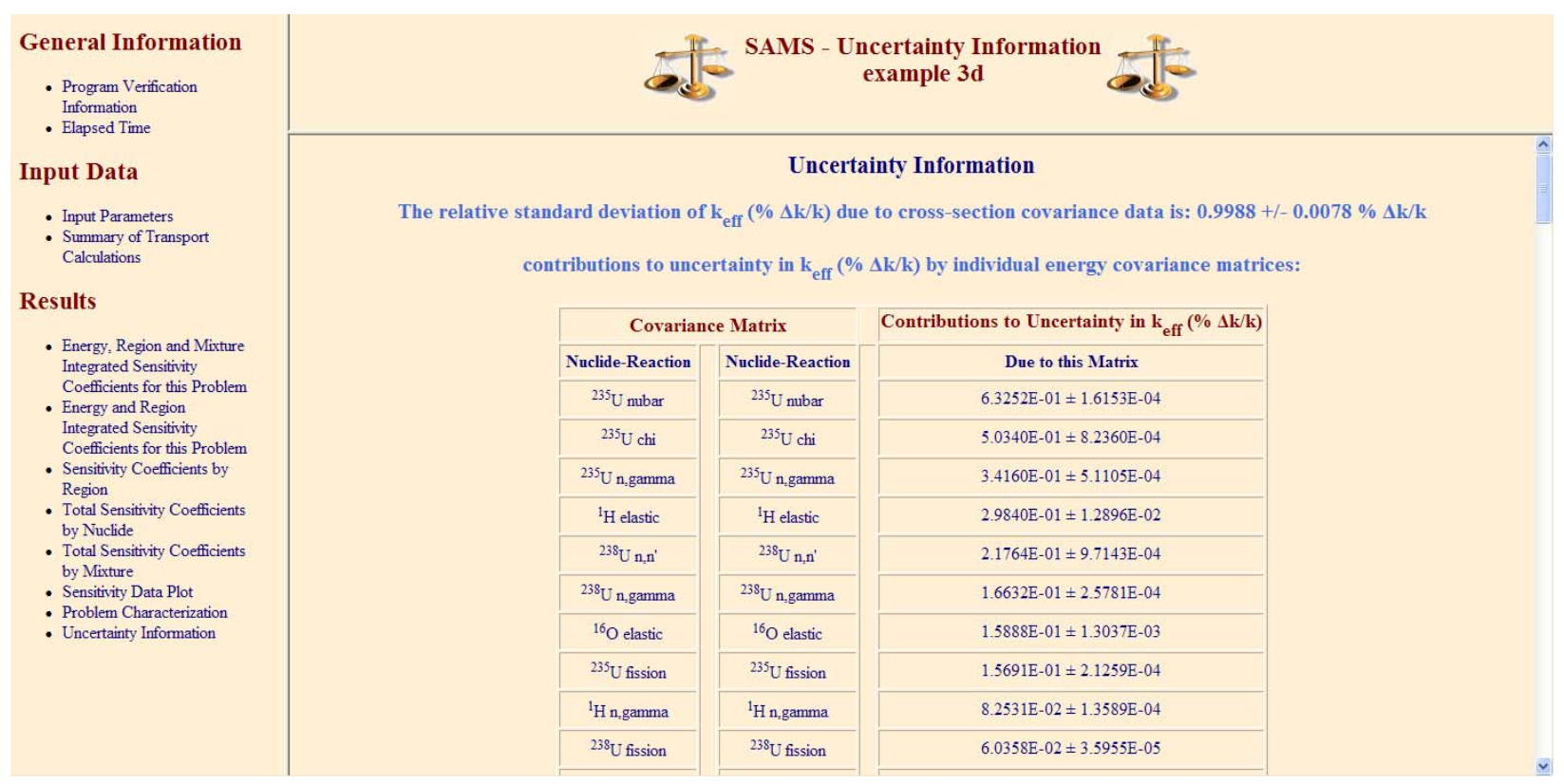

Fig. 49. Uncertainty information from TSUNAMI-3D example. 



\section{SELECTION OF BENCHMARK EXPERIMENTS}

As described in the previous sections, the accurate generation and interpretation of sensitivity and uncertainty data for individual systems can help the user gain insight into which processes are important and which ones may be the primary contributors to computational bias. TSUNAMI offers tools to use sensitivity and uncertainty information as a means of comparing systems to identify benchmarks experiments that are similar to the target application. The premise behind this concept is that computational biases are primarily due to errors in cross-sectional data. If a system has a high sensitivity in a given energy range to a cross section with large errors, the system is likely to exhibit a computational bias. If a benchmark experiment is identified that has a similar sensitivity to that of the application, the computational bias can be quantified with the benchmark, and the bias can be projected to the application system. This section describes how to use the TSUNAMI tools that assist in identifying important processes in the application system and finding benchmark experiments that can be useful in their validation.

\subsection{SENSITIVITY DATA DISTRIBUTED WITH ICSBEP HANDBOOK}

As described in previous sections, the generation of accurate sensitivity data with TSUNAMI can be time consuming. Because of the wide use of benchmark experiments from the International Criticality Safety Benchmark Evaluation Project (ICSBEP) Handbook for criticality safety validations, an effort is under way to distribute TSUNAMI sensitivity for many benchmarks through the ICSBEP. The handbook can be ordered, free of charge, at http://icsbep.inel.gov. For 2008 the ICSBEP included 281 SDFs generated by ORNL using SCALE 5.1 and the 238-group ENDF/B-VI.7 cross-section library that included the ENDF/B-VII. 0 cross section for ${ }^{233} \mathrm{U}$. To use the sensitivity data distributed with the 2008 edition of the handbook for comparision with application sensitivity data, the applications must be computed with the SAMS option unconstraintedchi for compatibility with the SCALE 5.1 data included in the ICSBEP. The files distributed with the ICSBEP were generated as part of a study published as Application of the SCALE TSUNAMI Tools for the Validation of Criticality Safety Calculations Involving ${ }^{233} U$, ORNL/TM-2008-196. 9 Distribution of additional SDFs is planned for subsequent releases of ICSBEP.

\subsection{EXAMPLE SYSTEM}

Because of the availability of SDFs for ${ }^{233} U$ experiments in the ICSBEP Handbook, a ${ }^{233} U$ system was selected to demonstrate the TSUNAMI methods for selection of benchmark experiments that are similar to the application system. The example system, referred to as Application 1 in ORNL/TM-2008-196, is a $12.2 \mathrm{~cm}$ radius sphere of $220 \mathrm{~g} \mathrm{U}$ per liter uranyl nitrate solution with no excess acid. The uranium is $100 \mathrm{wt} \%{ }^{233} \mathrm{U}$. The solution sphere is reflected by $0.25 \mathrm{~cm}$ of SS304 and $2 \mathrm{~cm}$ of water. The EALF calculated for this application is $0.282 \mathrm{eV}$. The $k_{\text {eff }}$ calculated for this system is $1.0028 \pm 0.0002$.

Sensitivity coefficients were computed for this system using TSUNAMI-3D from SCALE 5.1. However, the techniques presented for validation are applicable to data generated with SCALE 5.1 and/or SCALE 6. The TSUNAMI-3D input file for this case is shown in Fig. 50. 


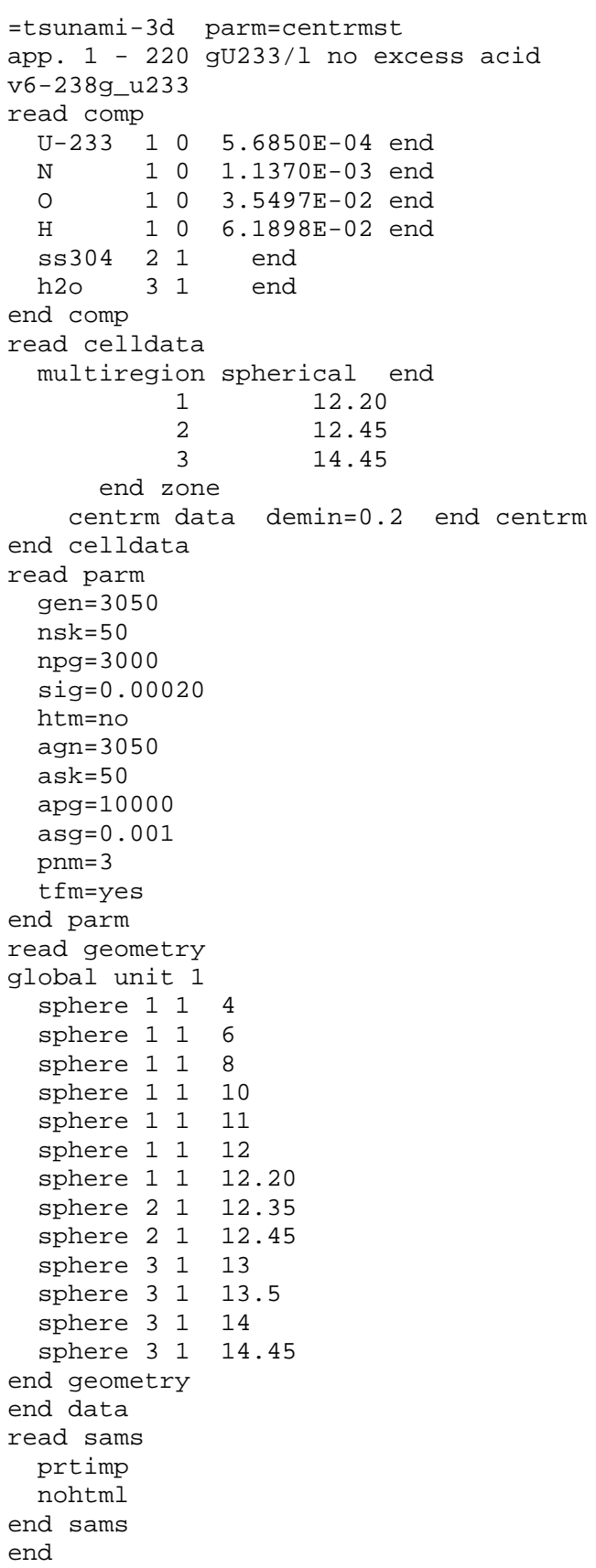

Fig. 50. TSUNAMI-3D input listing for ${ }^{233} \mathrm{U}$ Application 1.

\subsection{INTERPRETING SENSITIVITY DATA WITH VIBE}

VIBE (Validation, Interpretation and Bias Estimation) is a new graphical tool for SCALE 6 that assists with the interpretation of sensitivity data. Begin by launching VIBE from the SCALE 6 shortcuts. If you are running VIBE for the first time, you will likely encounter a dialog regarding the DICE (Database for the International Handbook of Evaluated Criticality Safety Benchmark Experiments) database location. DICE is distributed with the ICSBEP and provides a convenient means of extracting information regarding experiments from it. We will revisit DICE in a subsequent section. For now, simply click OK to dismiss the dialog. 
Next, we will load sample sensitivity data into VIBE. Begin by selecting Add database location... from the File menu as shown in Fig. 51.

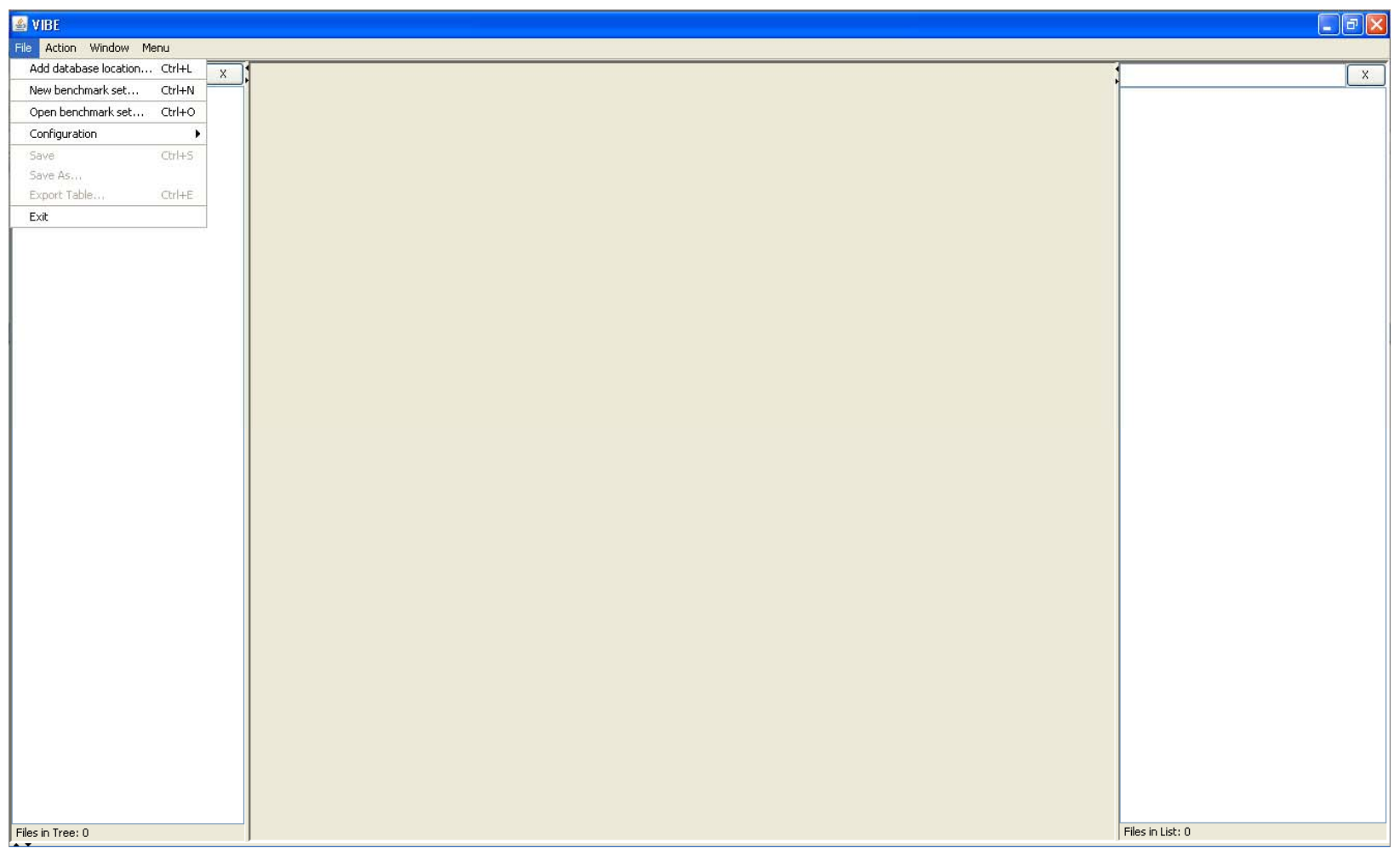

Fig. 51. VIBE with file menu.

In the file chooser that appears after selecting Add database location..., browse to the scale6 directory (usually on $\mathrm{C}$ : drive, but on $\mathrm{S}$ : drive on system used for this demonstration). In scale6, there is a directory sequence of primers, then tsunami, and then files. Inside of the files directory, the SDF for the ${ }^{233} \mathrm{U}$ application is provided. To mount this directory, click accept at the level where the files folder is showing, not the contents of the files folder. The proper location is shown in Fig. 52. 


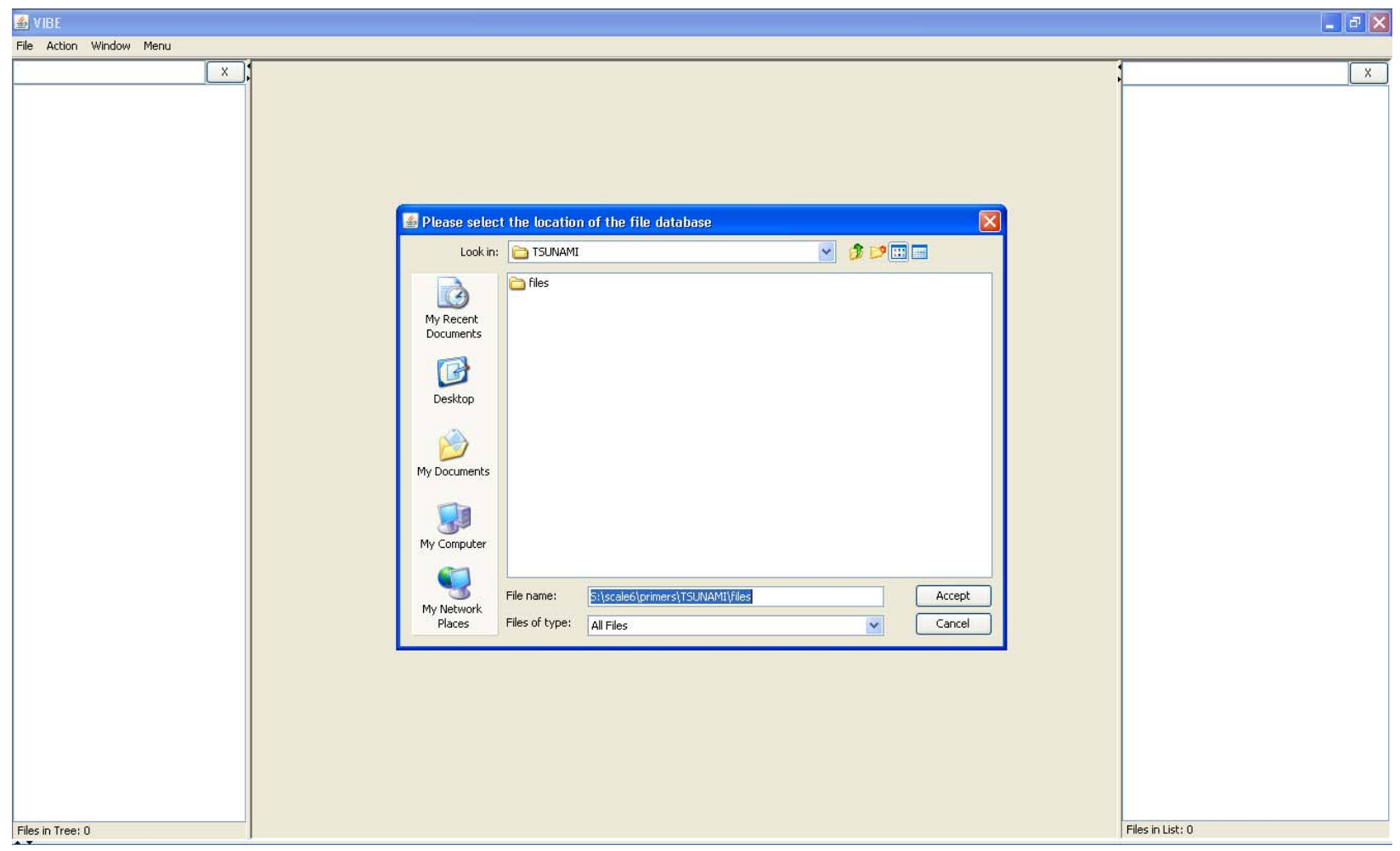

Fig. 52. Location of sample file for ${ }^{233} \mathrm{U}$ application.

The directory tree leading to the files directory will appear in the left pane of the VIBE application, as shown in Fig. 53. 


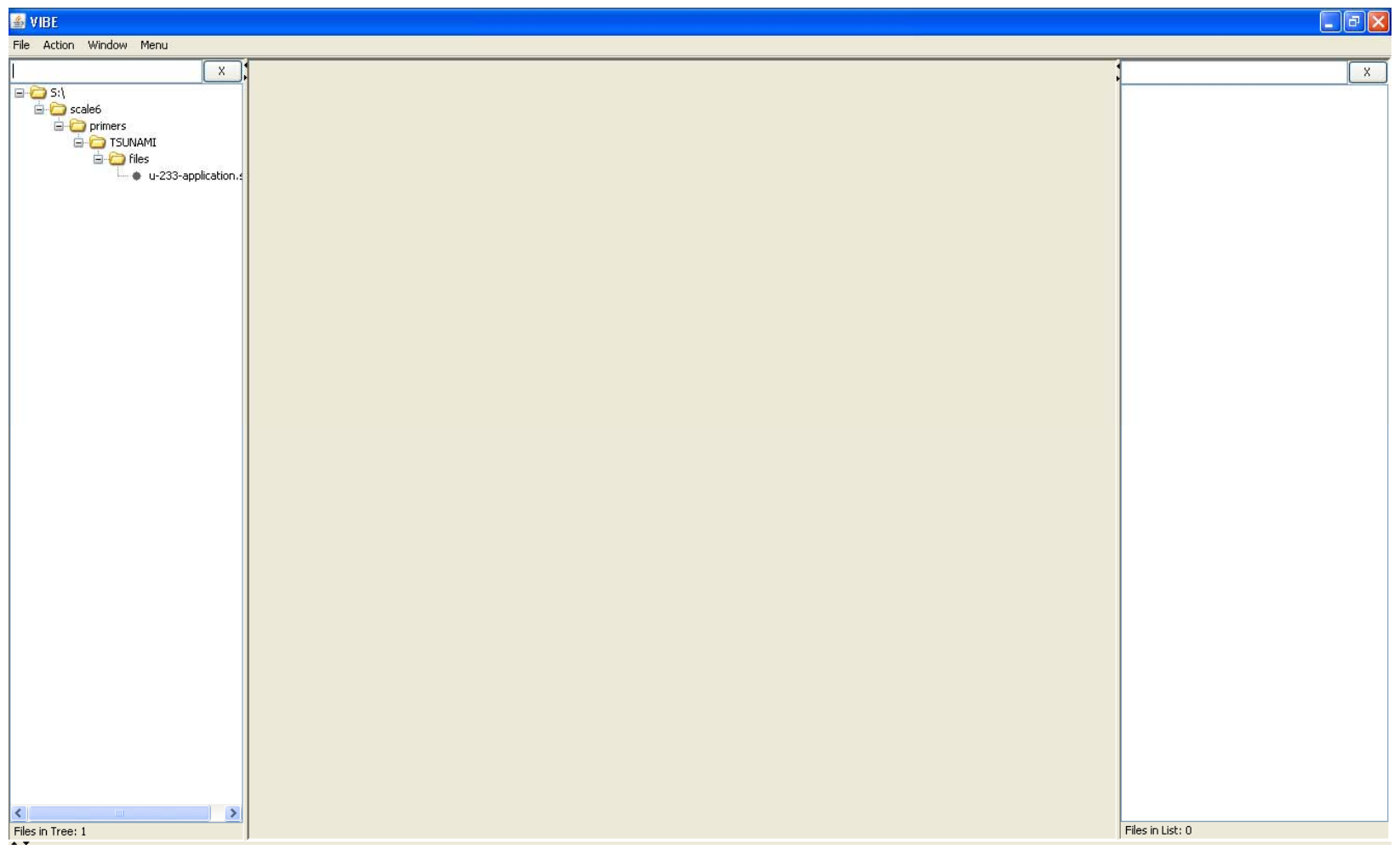

Fig. 53. VIBE application with directory tree.

A right-click on the SDF will open a pop-up menu with some options, as shown in Fig. 54. Select Open in Javapeno to view the TSUNAMI data in Javapeño. It may take several seconds for the Javapeño application to launch with the SDF loaded. Because Javapeño operates with separate windows, the VIBE application will still be visible after Javapeño loads, as shown in Fig. 55. 


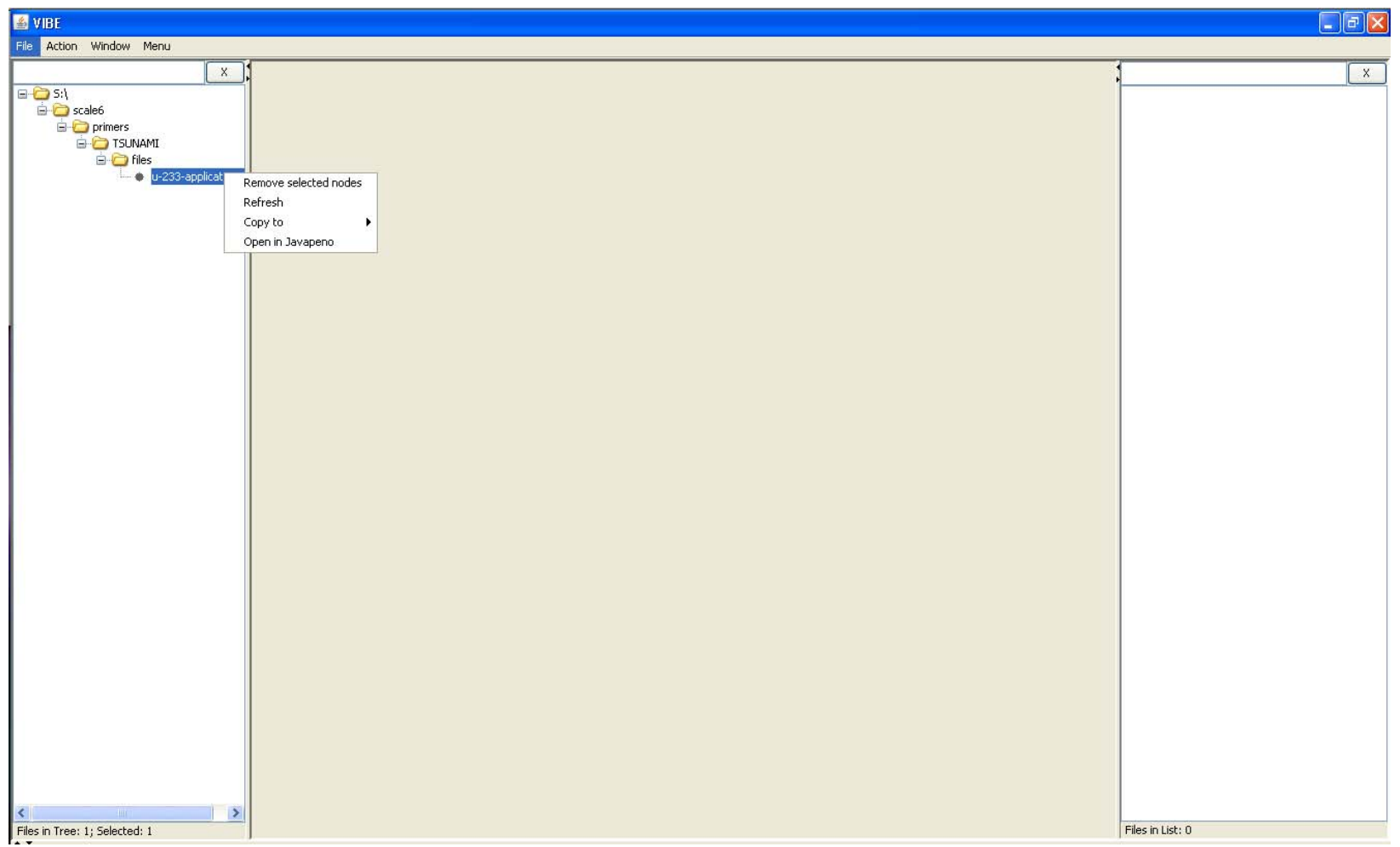

Fig. 54. VIBE application with SDF pop-up menu.

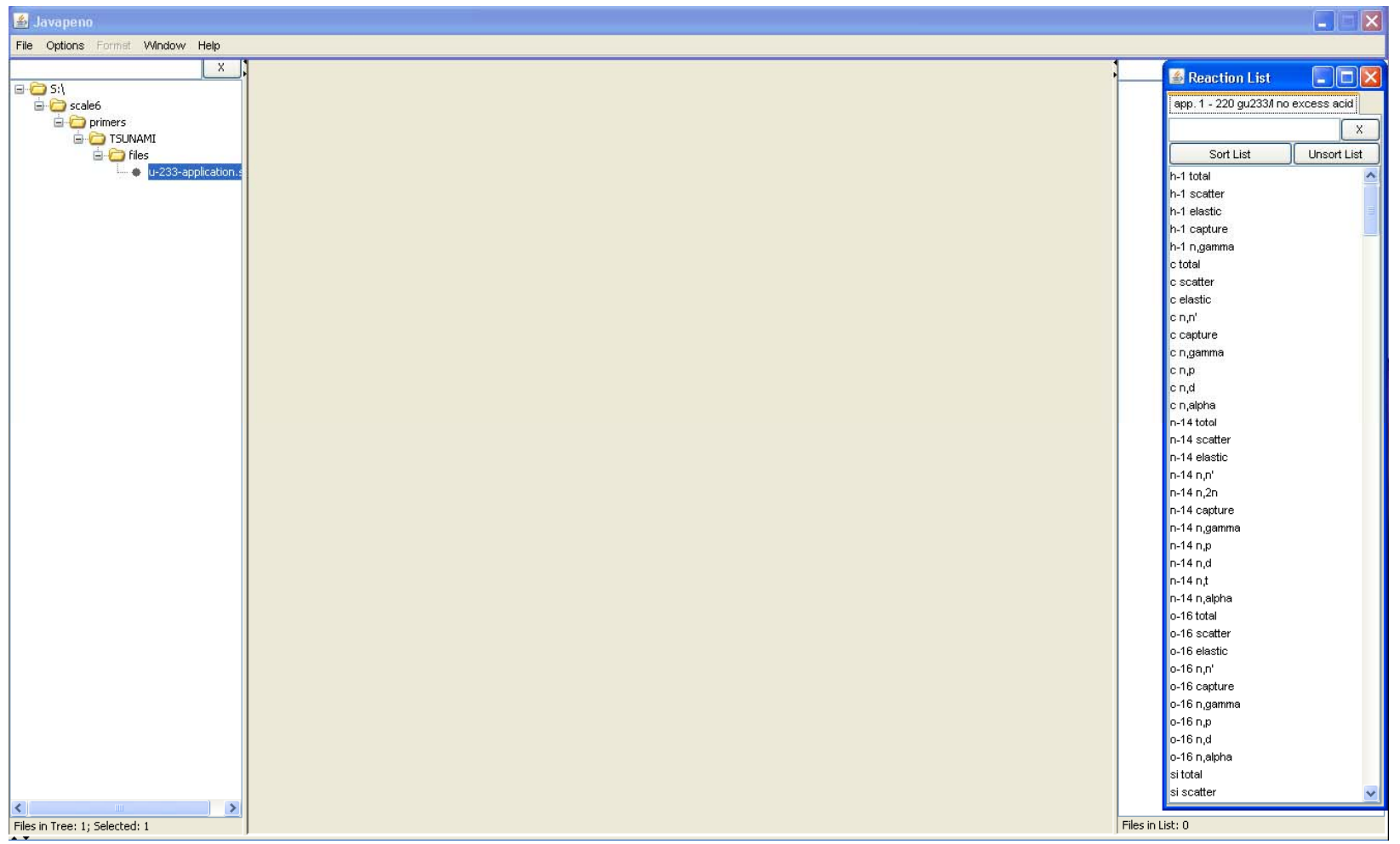

Fig. 55. View of Javapeño loaded over VIBE. 
Plots of the fission and n,gamma sensitivities for ${ }^{233} \mathrm{U}$ reveal a high sensitivity at thermal energies, as expected, and some sensitivities at intermediate energies.

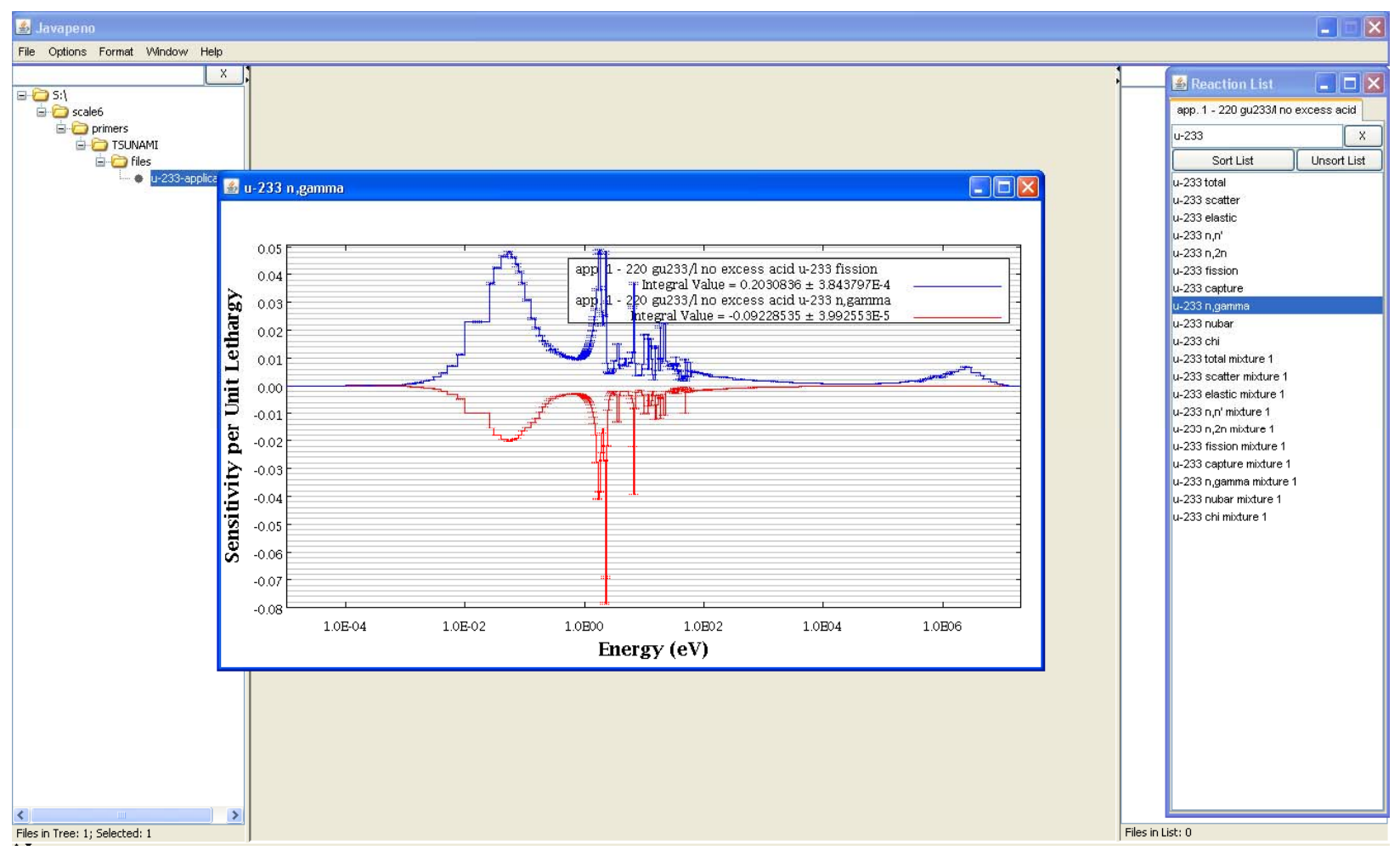

Fig. 56. Plotting ${ }^{233} \mathrm{U}$ fission and $\mathrm{n}$,gamma for ${ }^{233} \mathrm{U}$ application.

Performing a group collapse of the sensitivity data and applying VIBE's sorting and filtering capabilities will assist in identifying important processes in this system. To manipulate sensitivity data, the SDFs of interest must first be gathered in the VIBE benchmark set. For this example, the SDF for the ${ }^{233} \mathrm{U}$ application will be the only file in the set. VIBE provides two ways to start a new set. One way is to right-click on the SDF and select Copy to and then New Benchmark Set, as shown in Fig. 57. The other way is to simply drag and drop the SDF from the left pane to the center pane of VIBE. With either method, a dialog will appear requesting the name of the new set. Enter $u-233$ application and click OK, as shown in Fig. 58. 


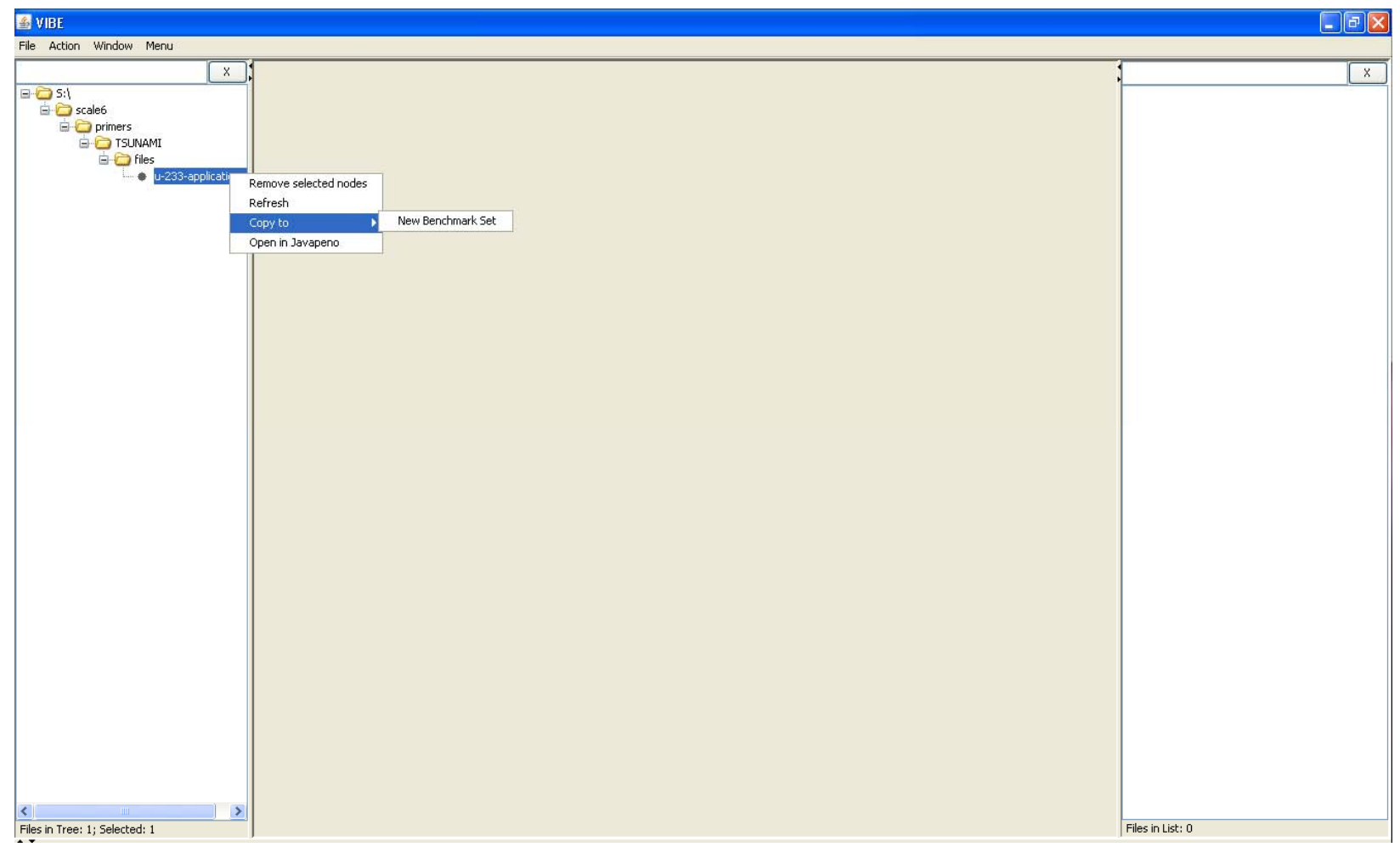

Fig. 57. Creating a new benchmark set in VIBE.

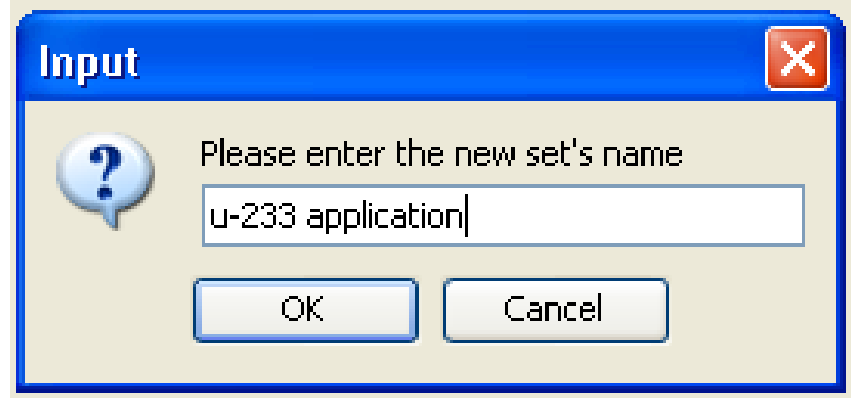

Fig. 58. Benchmark set name dialog.

At this point, VIBE will add a new window inside the center benchmark set pane, and the right list pane will show the full directory path to the SDF, as shown in Fig. 59. If more than one benchmark set is available, VIBE operations are conducted on the currently selected benchmark set window. 


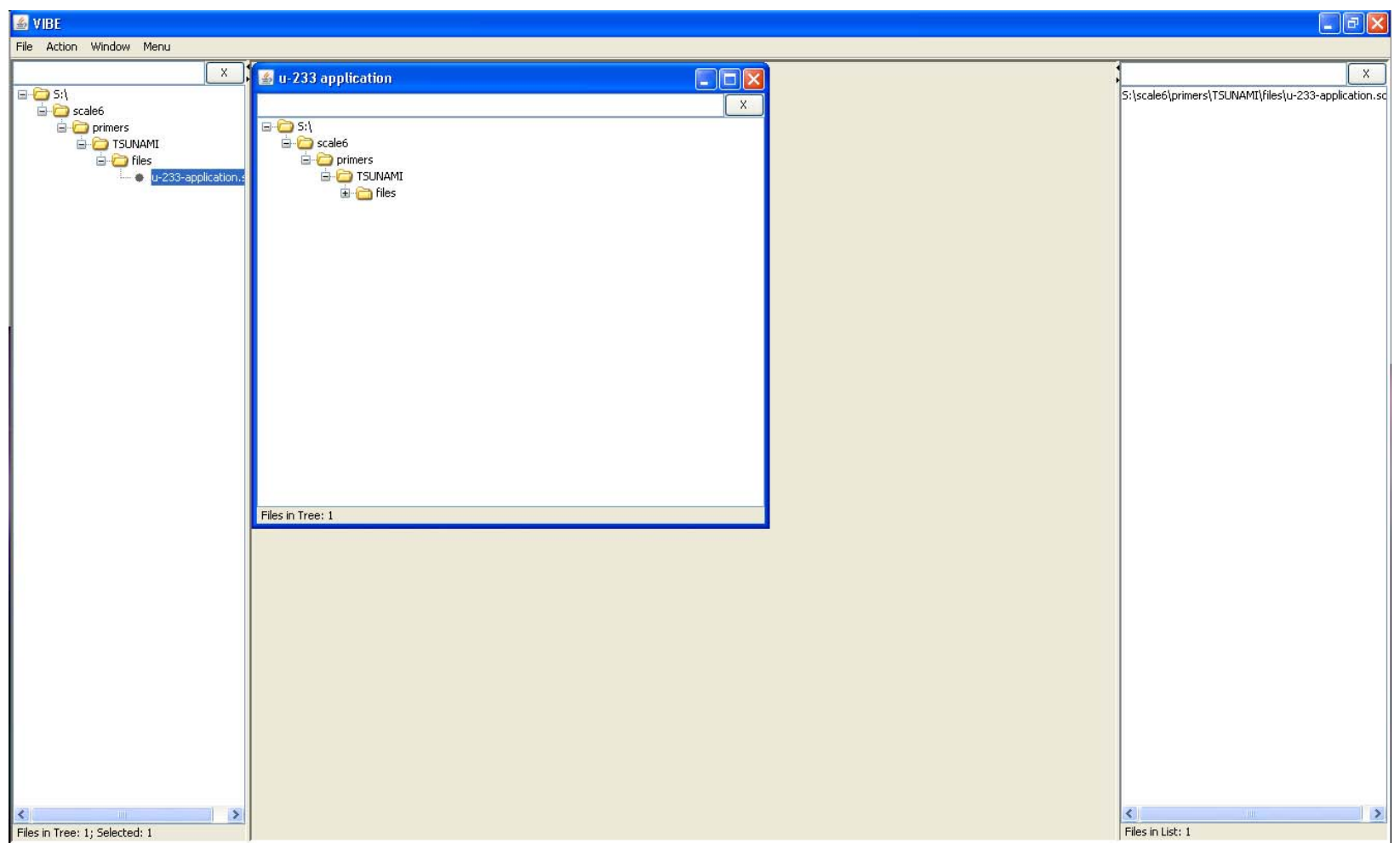

Fig. 59. VIBE with new benchmark set.

With a simple benchmark set established, a group collapse of the sensitivity data can be performed. Select Collapse Benchmark Set... from the Action menu, as shown in Fig. 60. In the Collapse Benchmark Set dialog, the boundaries for the group collapse can be selected by hand by clicking on the checkbox next to each desired boundary, or they can be set automatically using predefined group structures. The group structures available are 3-, 4-, 44- and 238-group. By clicking the checkbox next to Use Predefined Structures, the 3-group structure, consistent with the ICSBEP 3-group boundaries, is selected. Other group structures can be selected from the drop-down menu, but in this example, the 3-group structure is used. Once this is selected, as shown in Fig. 61, click Collapse. 


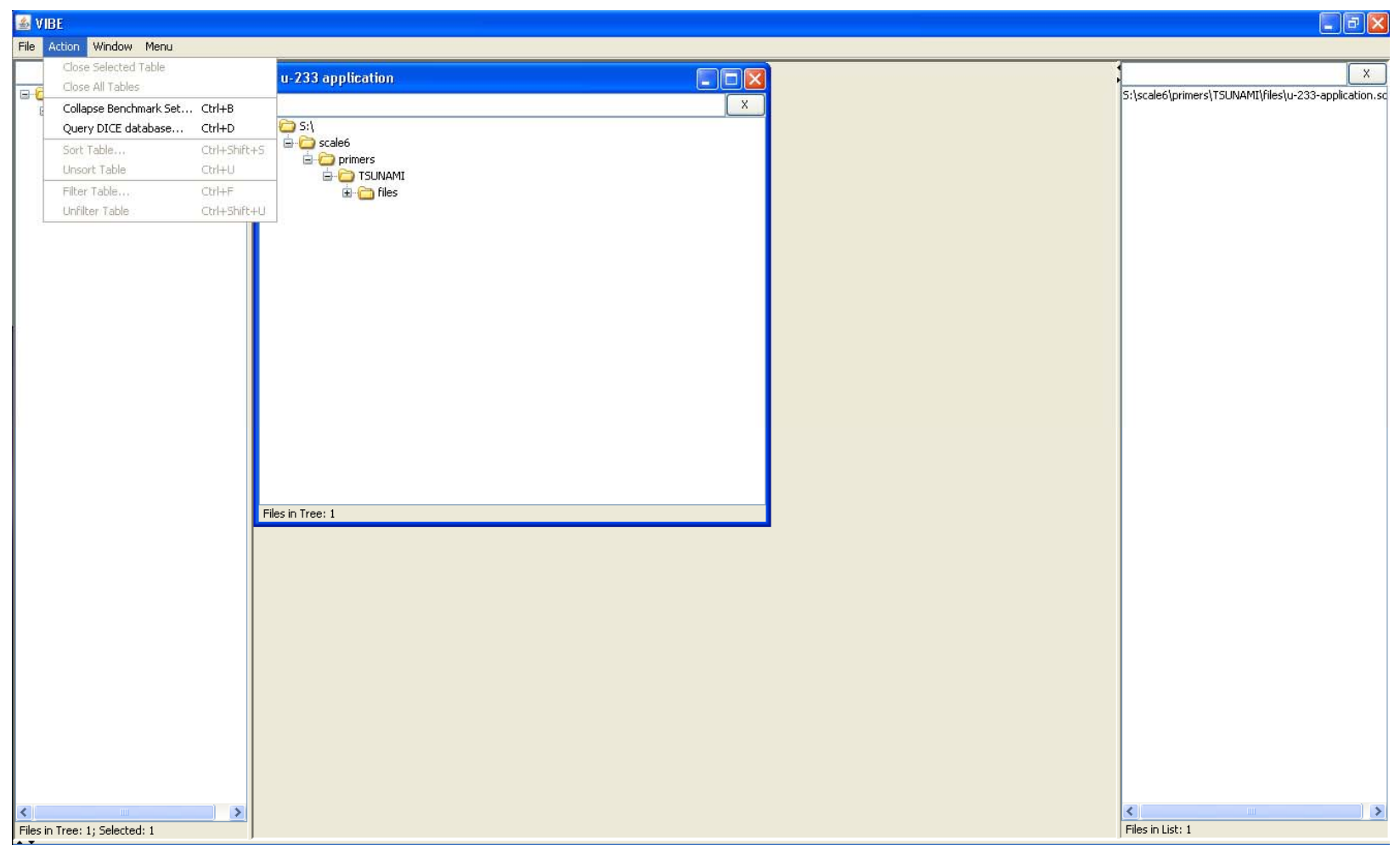

Fig. 60. VIBE action menu.

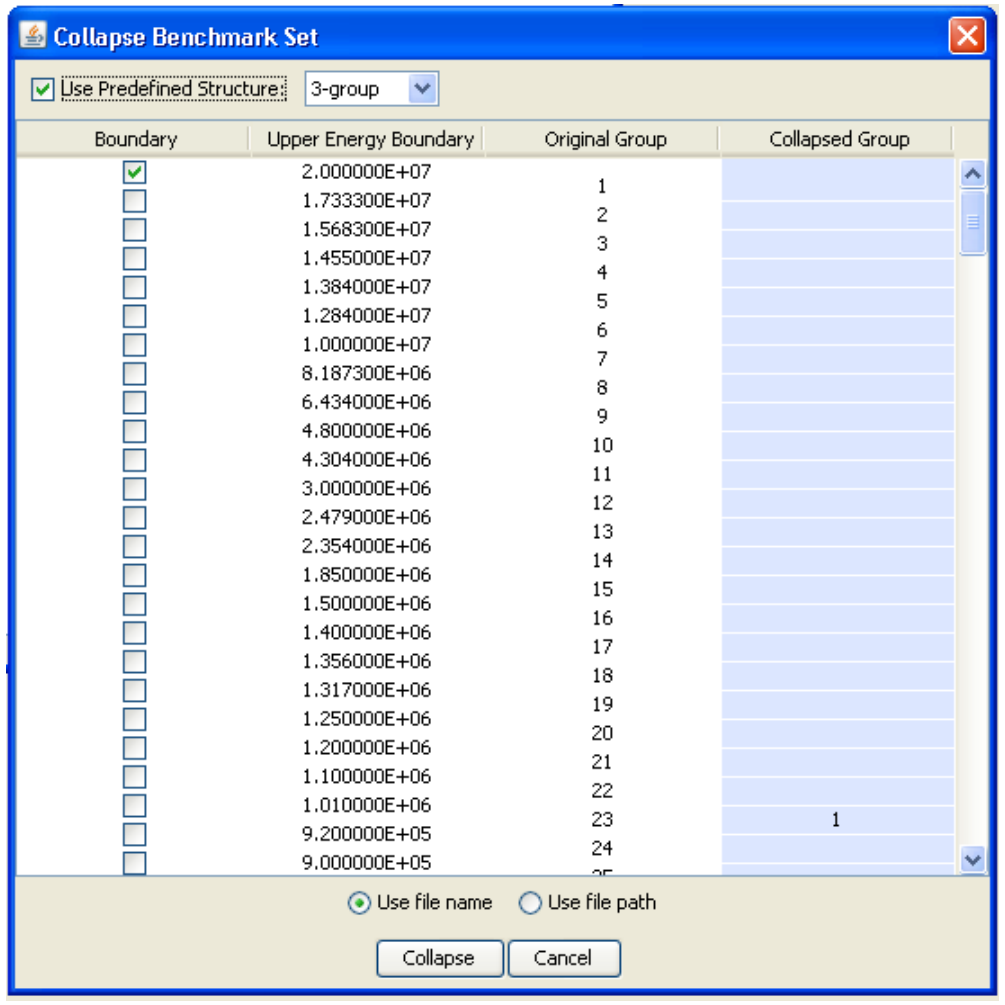

Fig. 61. VIBE group collapse dialog. 
A new table will appear with the collapsed data, as shown in Fig. 62. The collapsed table contains columns for row number, SDF identifier, computed $k_{\text {eff }}$ and its uncertainty, nuclide and reaction for the sensitivity data, and 3-group sensitivity data and their uncertainties. The energy range for each collapsed group is shown at the top of the columns, and the statistical uncertainties due to the TSUNAMI-3D Monte Carlo calculations are shown for each group in the column labeled Std. Dev. The table contains a row for the nuclide-reaction pair for which sensitivity data are available on the SDF. For this file, there are 199 rows in the table, as shown in the lower left corner of the table pane. With so many rows, it may be difficult to visually inspect the data to identify the highest sensitivities for which validation data are desired. To simplify this process, the VIBE sorting capabilities will be used.

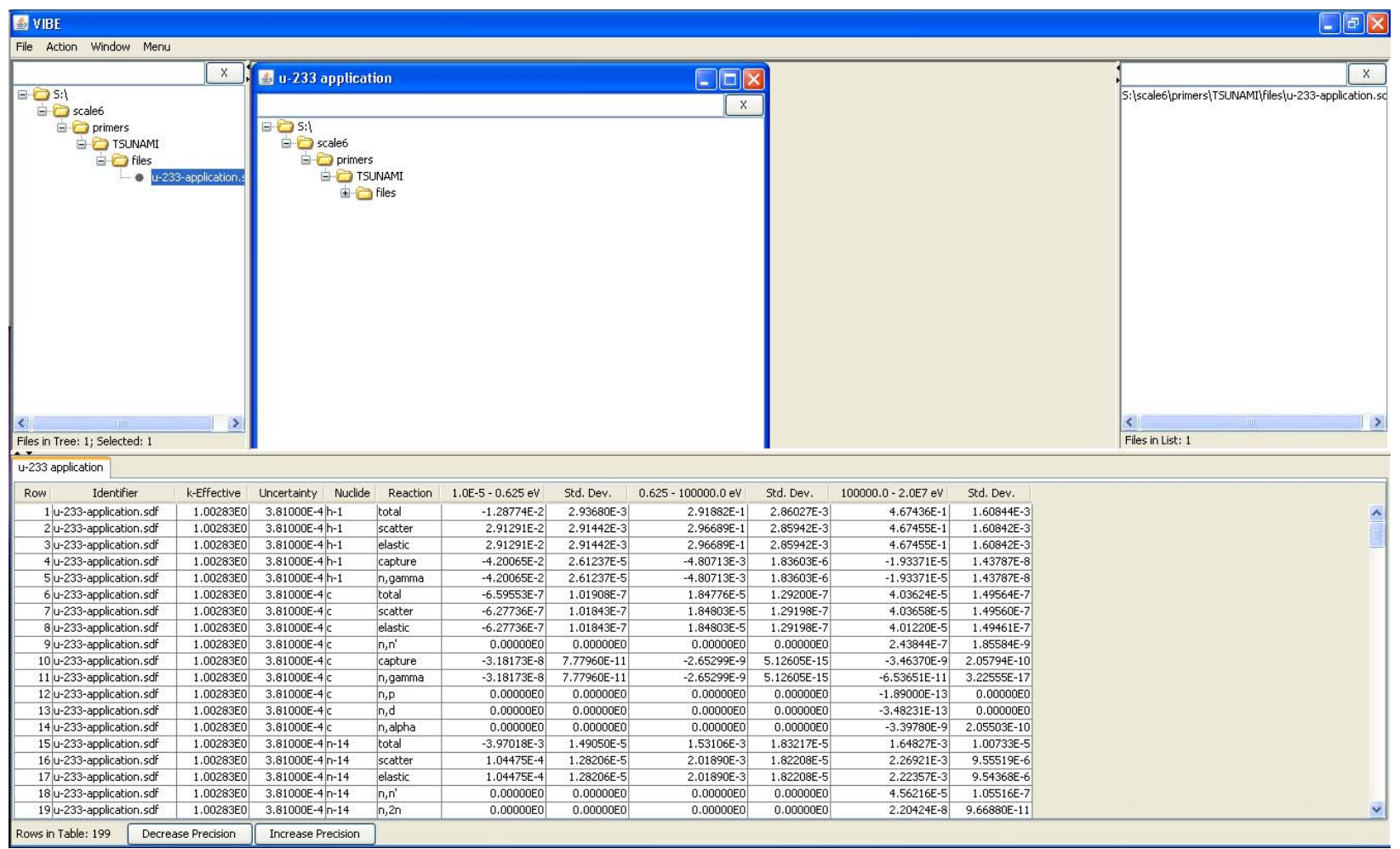

Fig. 62. VIBE with group collapsed sensitivity data.

When a table is showing in VIBE, the Action menu will present items to manipulate the currently selected table by closing, sorting, or filtering. To identify nuclide-reaction pairs with high sensitivities, select Sort Table.... Begin by sorting thermal data in descending order so that the most positive sensitivities will be at the top of the table and the most negative will be at the bottom by setting the Sort dialog as shown in Fig. 64 and clicking Sort. 


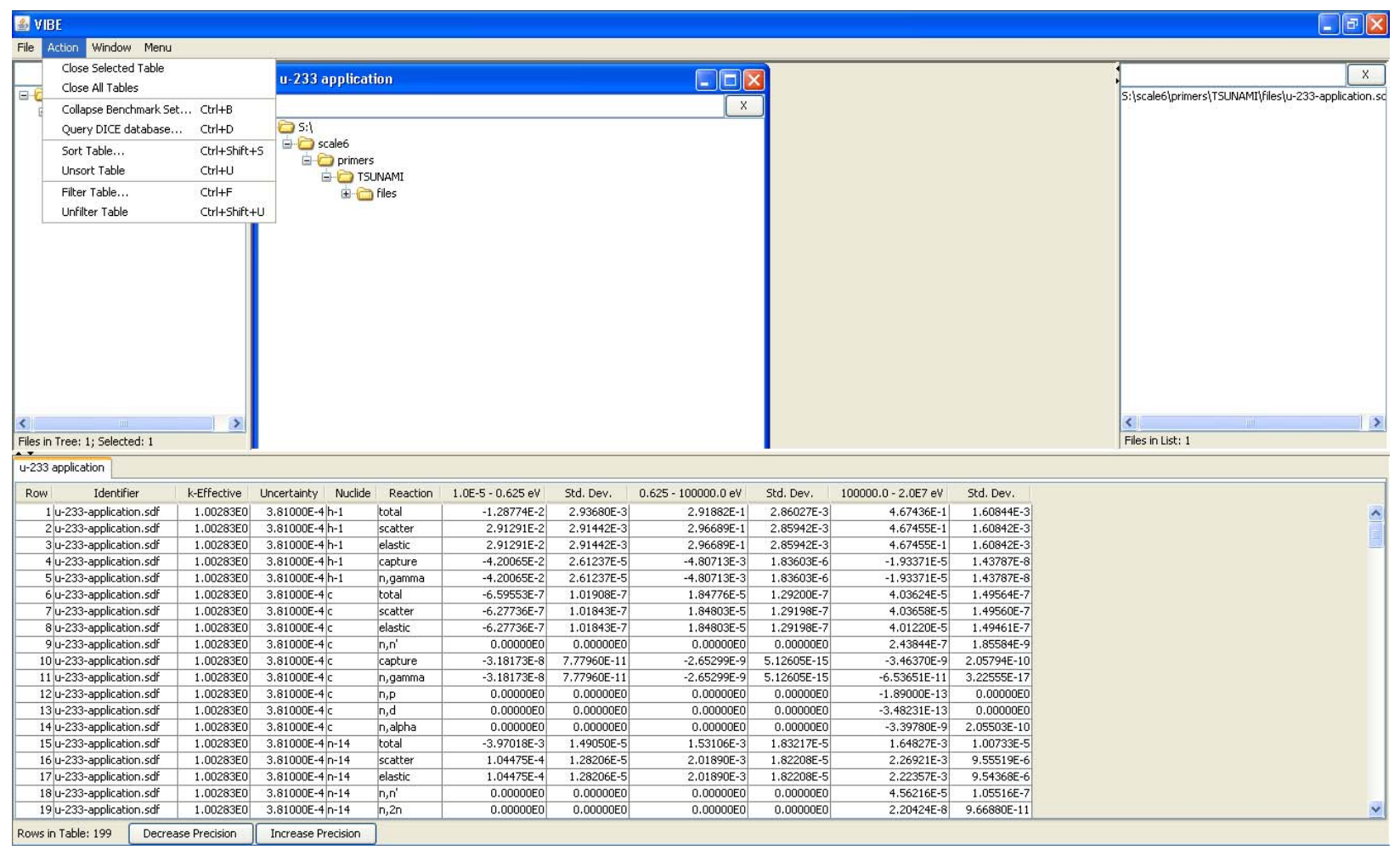

Fig. 63. VIBE action menu with table showing.

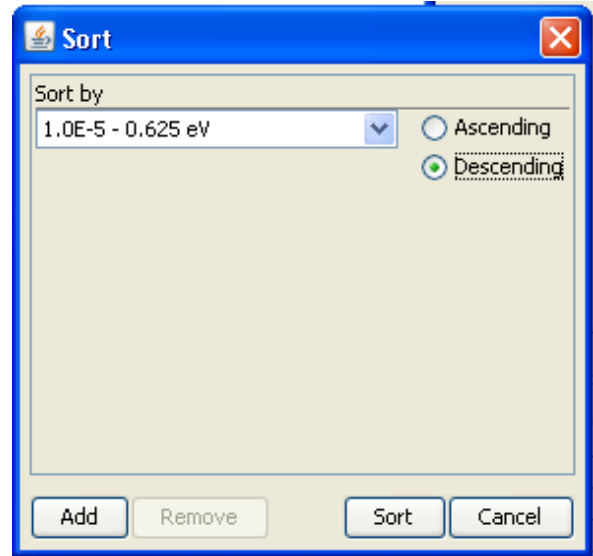

Fig. 64. VIBE sort dialog for sorting by thermal sensitivities in descending order.

The sorted sensitivities appear as shown in Fig. 65. As expected, the ${ }^{233} \mathrm{U}$ sensitivities have the highest positive values followed by ${ }^{1} \mathrm{H}$ and ${ }^{16} \mathrm{O}$. It is also important to check the bottom of the table for sensitivities with large negative values, as shown in Fig. 66. Here the capture reactions of ${ }^{233} \mathrm{U}$ and ${ }^{1} \mathrm{H}$ have the largest magnitudes, followed by ${ }^{1} \mathrm{H}$. The next most negative isotope is ${ }^{56} \mathrm{Fe}$, but its values are an order of magnitude smaller than those of ${ }^{233} \mathrm{U}$ and ${ }^{1} \mathrm{H}$. Thus, it is expected to be of secondary importance for validation. 


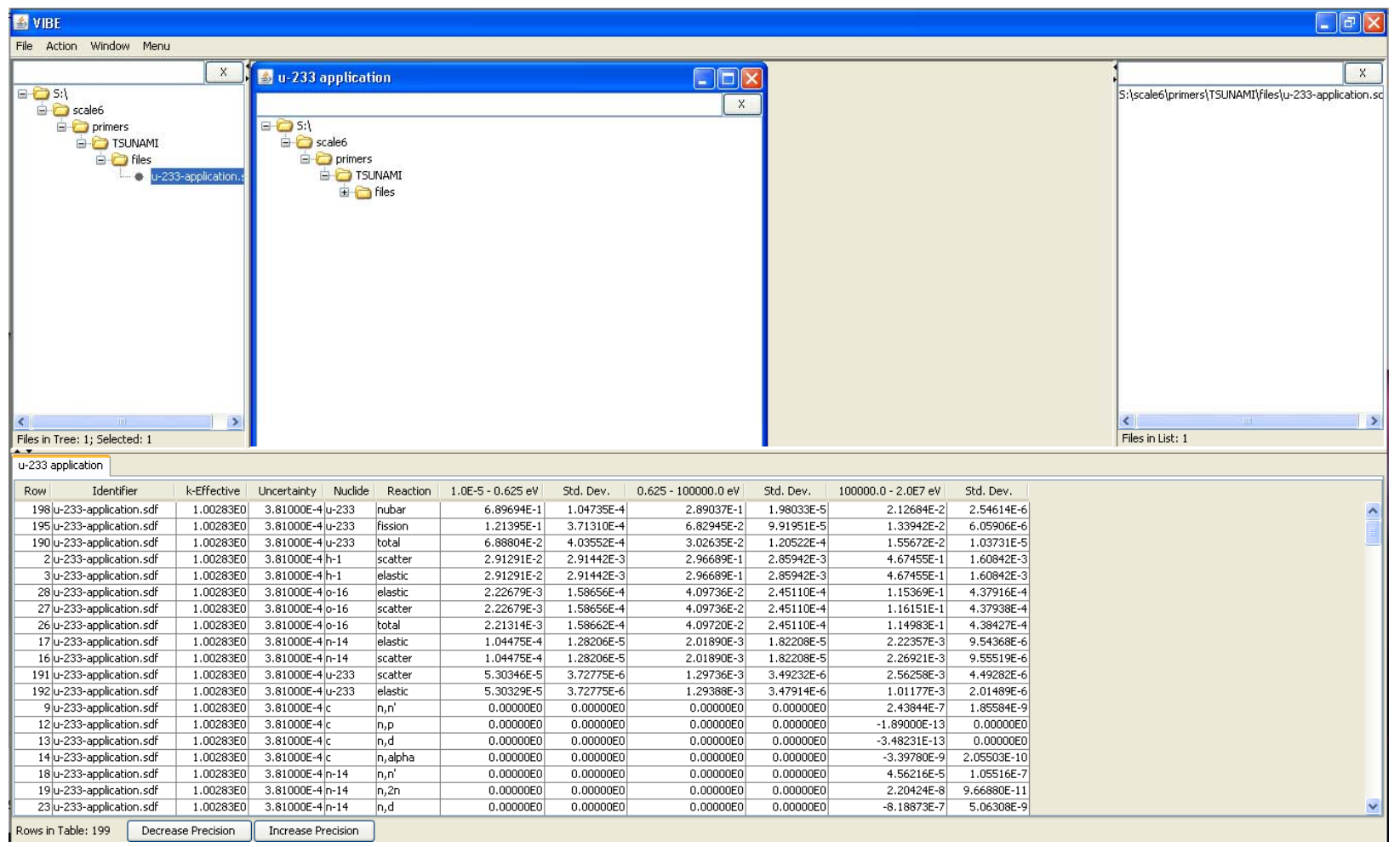

Fig. 65. VIBE with thermal sensitivity data sorted in descending order.

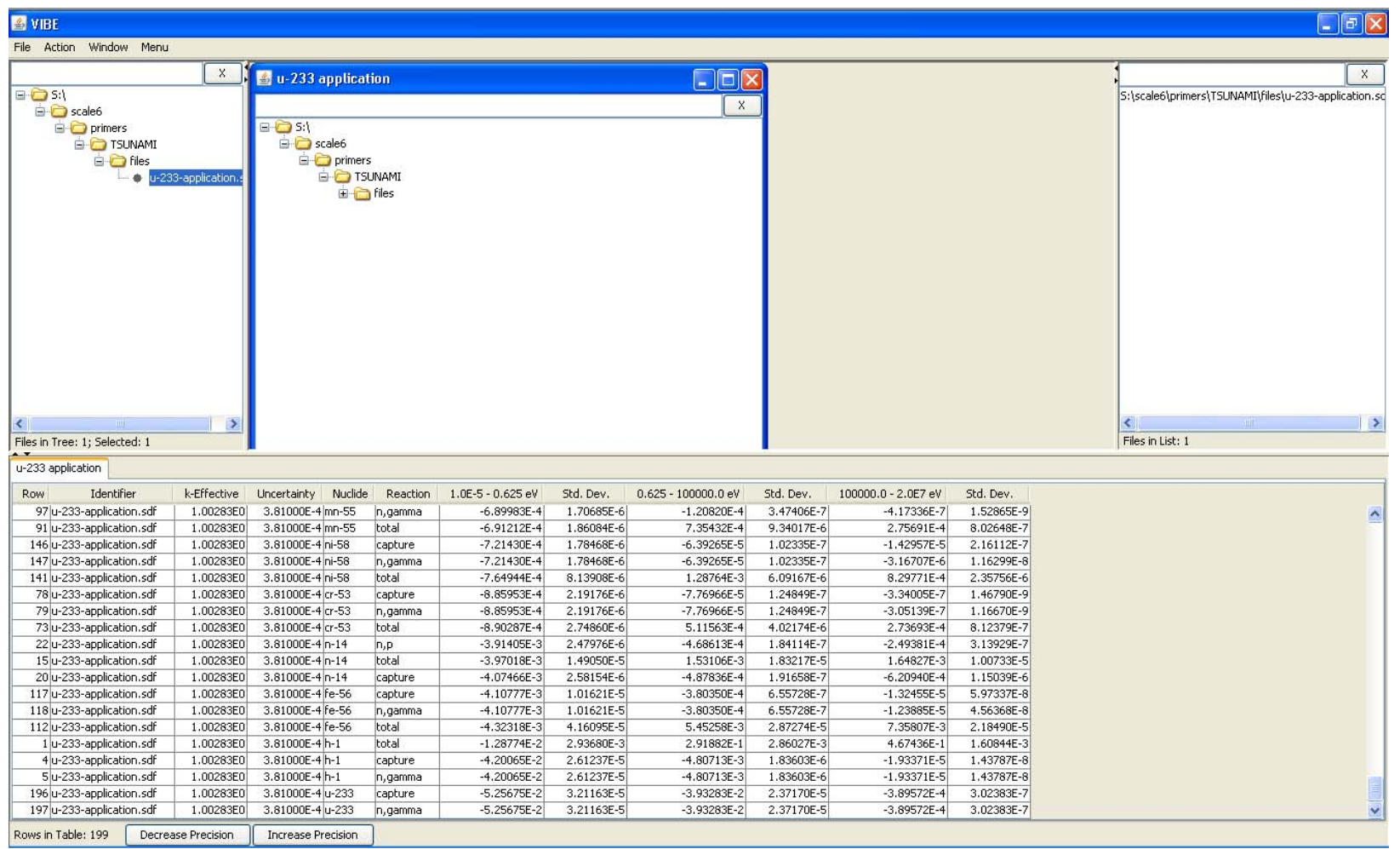

Fig. 66. VIBE showing bottom of table with thermal sensitivity data sorted in descending order. 


\subsection{INITIAL SCREENING OF AVAILABLE EXPERIMENTS WITH VIBE}

To begin comparing the sensitivity data for the application with the sensitivities of benchmarks from the ICSBEP Handbook, insert the handbook DVD in the DVD drive, select Add Database Location... from the VIBE File menu, and browse to the "Handbook" DVD. Select the folder called Dice, as shown in Fig. 67, and select Accept.

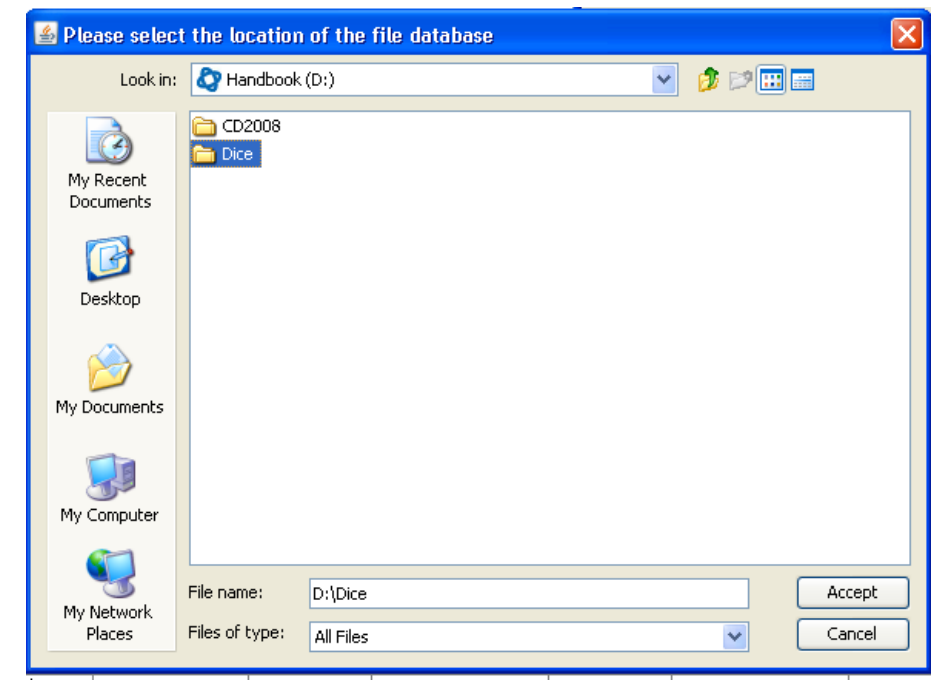

Fig. 67. VIBE file chooser to select TSUNAMI data distributed in ICSBEP Handbook.

VIBE will read the handbook DVD and mount the directory containing the TSUNAMI sensitivity data in the directory tree in the left pane of VIBE. Open the new tree to view the sensitivity files, as shown in Fig. 68. Selecting the ornl folder will reveal in the status bar at the bottom of the pane that there are 281 files contained in the selected folder and 282 total files in the directory tree. The one additional file is the application file previously loaded.

Create a new benchmark set to examine the benchmark sensitivity data by dragging the ornl folder to the empty space in the center benchmark set pane, next to, but not in, the window called u-233 application, as shown in Fig. 69. A dialog will appear requesting the name of the new benchmark set; enter handbook, as shown in Fig. 70. 


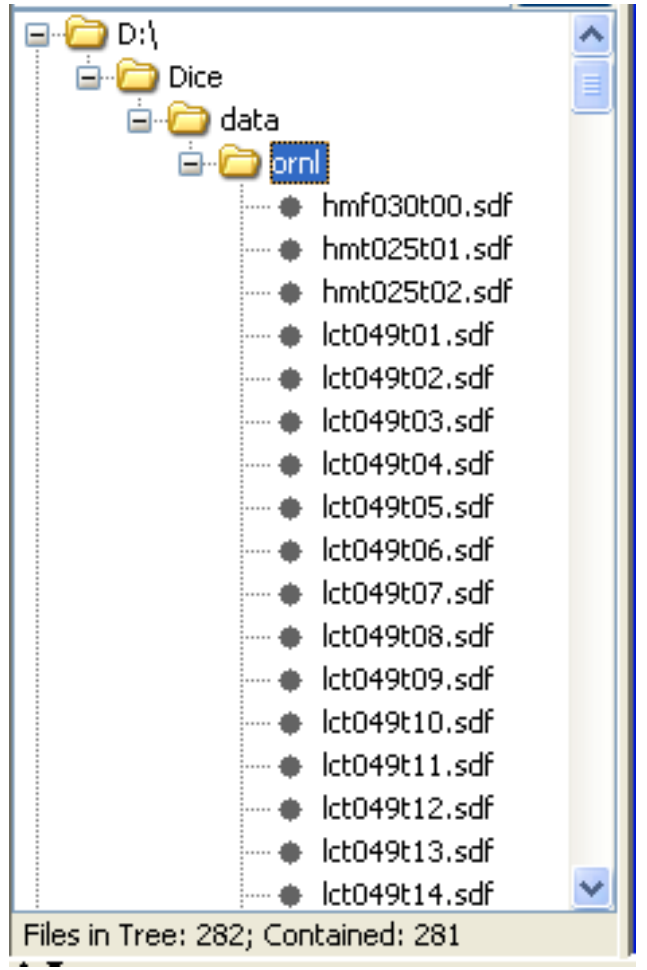

Fig. 68. VIBE file tree pane with ICSBEP TSUNAMI sensitivity data selected.

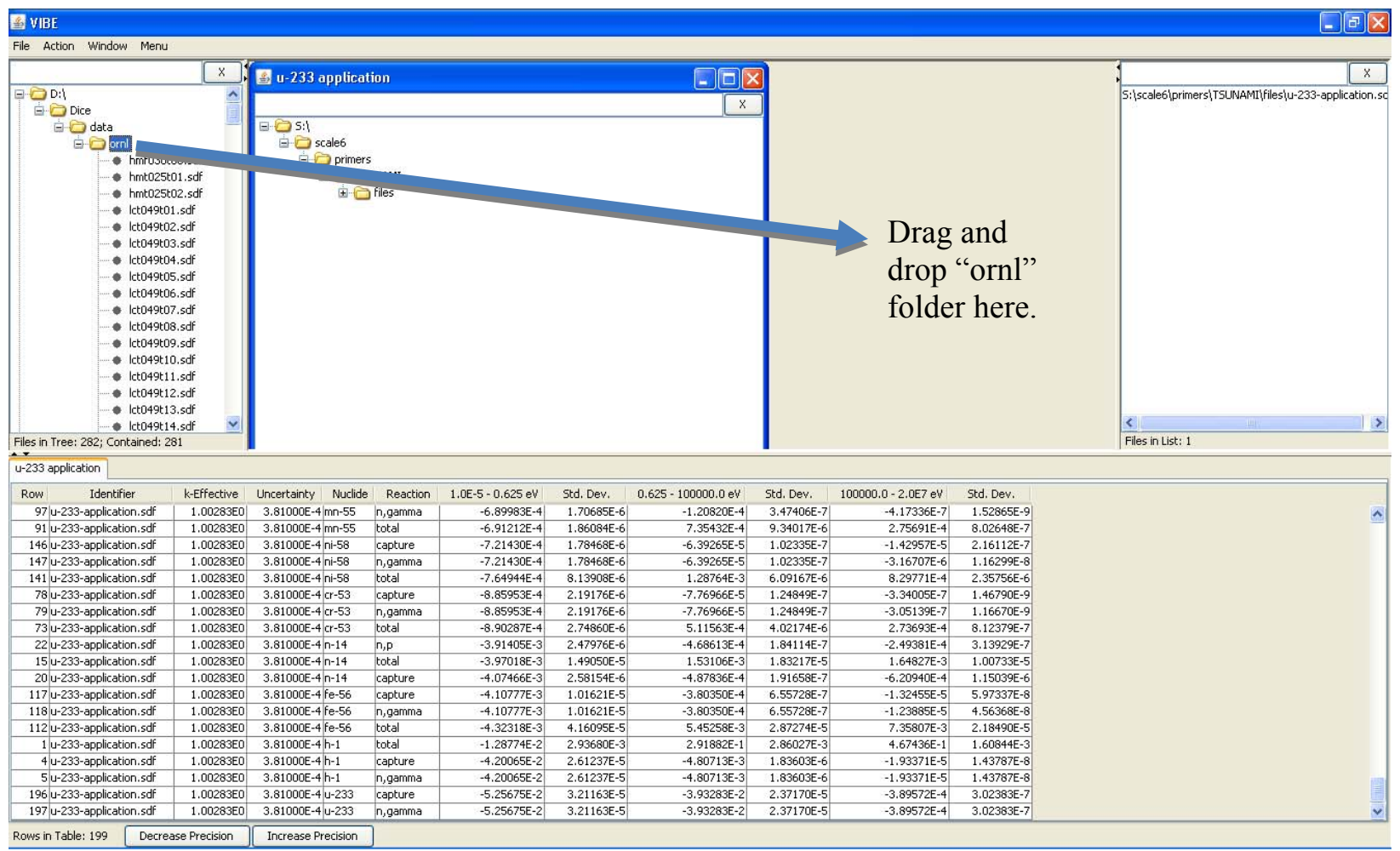

Fig. 69. Creation of new benchmark set for data distributed with ICSBEP Handbook. 


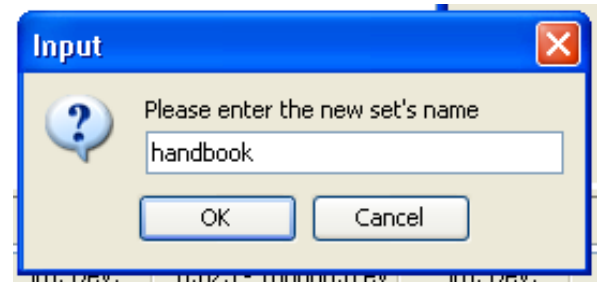

Fig. 70. VIBE benchmark set dialog for ICSBEP Handbook data.

Collapsing the sensitivity data in the same group structure as the application will allow identification of experiments with sensitivities, and thus computational biases, similar to those of the application. To perform a group collapse on all experiments in the handbook set, repeat the same process as was previously performed for the $\mathrm{u}-233$ application set. With the handbook benchmark set window selected, select Collapse Benchmark Set... from the Action menu, in the Collapse Benchmark Set dialog, check the box to use the predefined 3-group structure, and click Collapse. The collapse of the 281 files from the handbook DVD may take several seconds to complete. The procedure will likely occur faster if the SDFs from the handbook are first copied to a local hard drive. The resulting collapsed data will appear as shown in Fig. 71. Note at the lower left corner of the table that the status line states there are 59,661 rows in this collapsed table. The number of rows corresponds to the number of sensitivity profiles in all 281 SDFs considered. Note that the initial order of the table corresponds to the order of the SDFs in the benchmark set and the order of the profiles within each SDF.

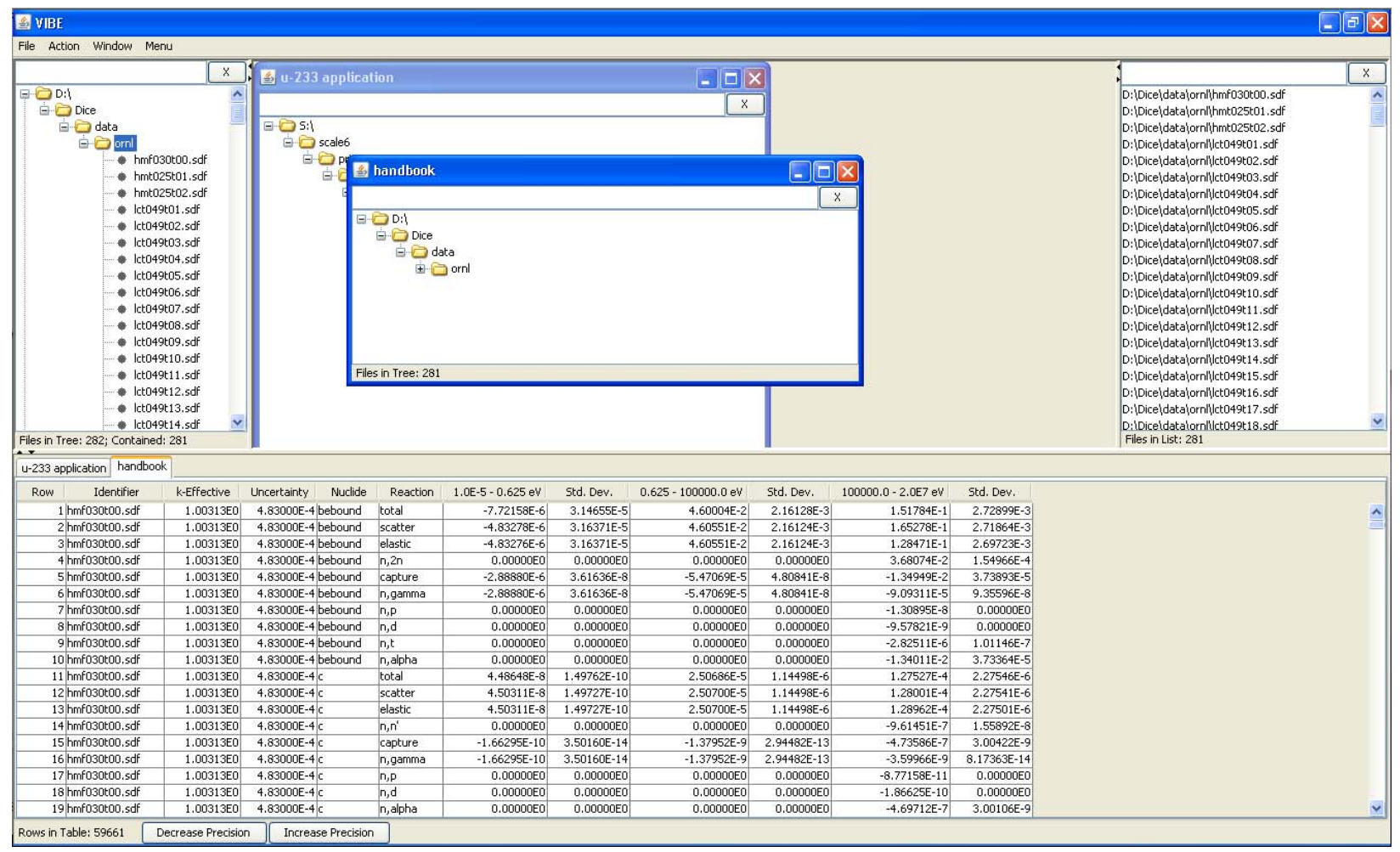

Fig. 71. VIBE with group collapsed sensitivity data for benchmarks in the ICSBEP Handbook. 
To examine the availability of sensitivities in the benchmarks that are similar to those in the application, VIBE's sorting and filtering capabilities will be used. As shown in Fig. 65, the application is most sensitive to ${ }^{233} \mathrm{U}$ nubar in the thermal range. Begin by filtering the benchmarks to eliminate all other reactions except ${ }^{233} \mathrm{U}$ nubar. With the handbook table showing, select Filter Table... from the Action menu. In the Filter dialog, select Nuclide from the first pull-down menu, select is from the second pull-down menu, and type $u$-233 in the specification field. Next, click Add Constraint, and set the next constraint for reaction is nubar. Ensure that All of These is selected at the bottom of the dialog so it appears as shown in Fig. 72, and then select Basic Filter to apply the filter to the table.

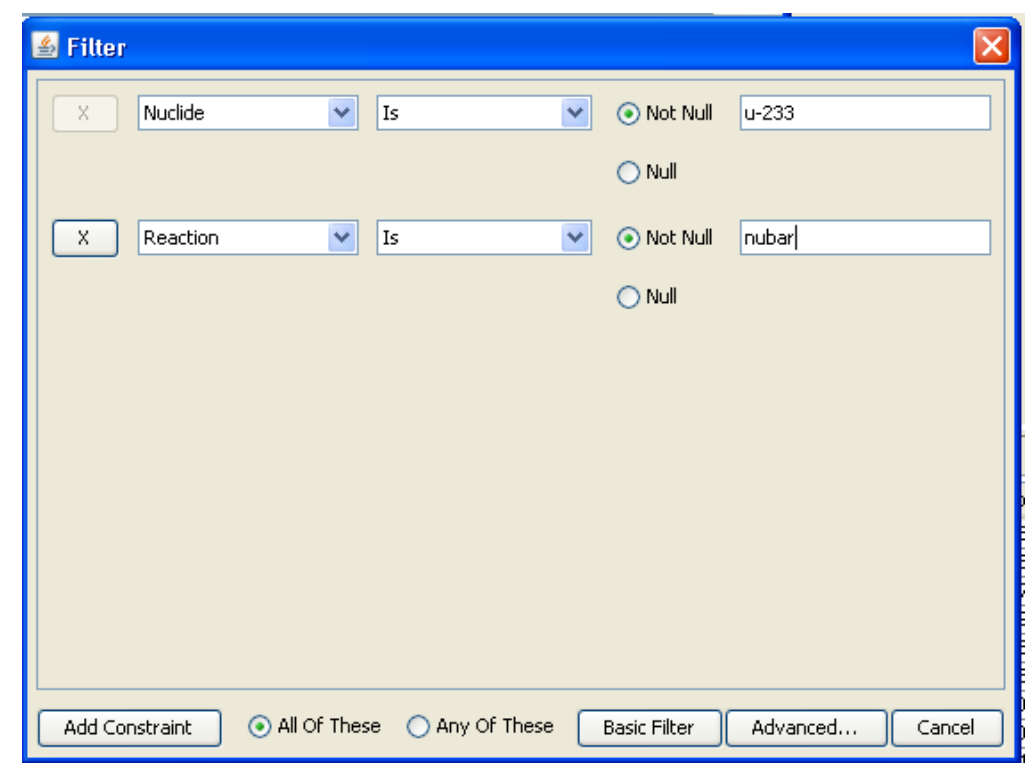

Fig. 72. VIBE filter dialog for ${ }^{233} \mathrm{U}$ nubar.

The filtered table will appear as shown in Fig. 73. Note that there are now only 232 rows in the table. Sorting the table by the thermal sensitivity in descending order provides the results shown in Fig. 74. Note that the highest ${ }^{233} \mathrm{U}$ nubar thermal sensitivities are $>0.95$, where the application sensitivity is 0.69 , as shown in Fig. 65. Although high coverage is provided by these benchmarks, the benchmarks with the highest thermal sensitivities may exaggerate the bias due to ${ }^{233} \mathrm{U}$ at thermal energies, while underrepresenting important processes at other energies or in other nuclides and reducing similarity to the application system. Scrolling down through the table, it can be observed that there are many benchmarks with ${ }^{233} \mathrm{U}$ nubar thermal sensitivities near 0.69 , as shown in Fig. 75 . The number of benchmarks with sensitivities near 0.69 can be determined using VIBE's filter capabilities. 


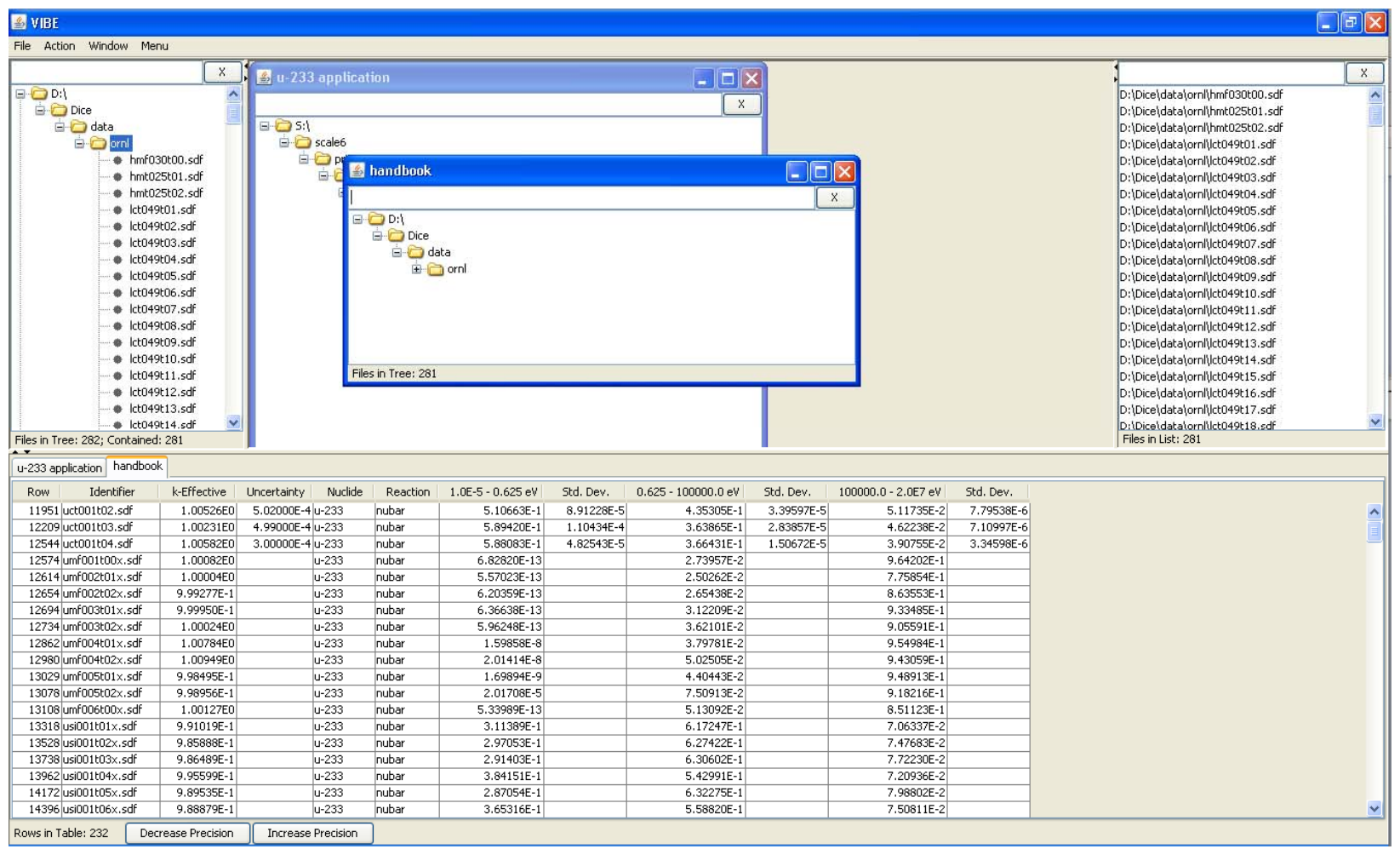

Fig. 73. VIBE with ${ }^{233} \mathrm{U}$ filter applied to ICSBEP Handbook data.

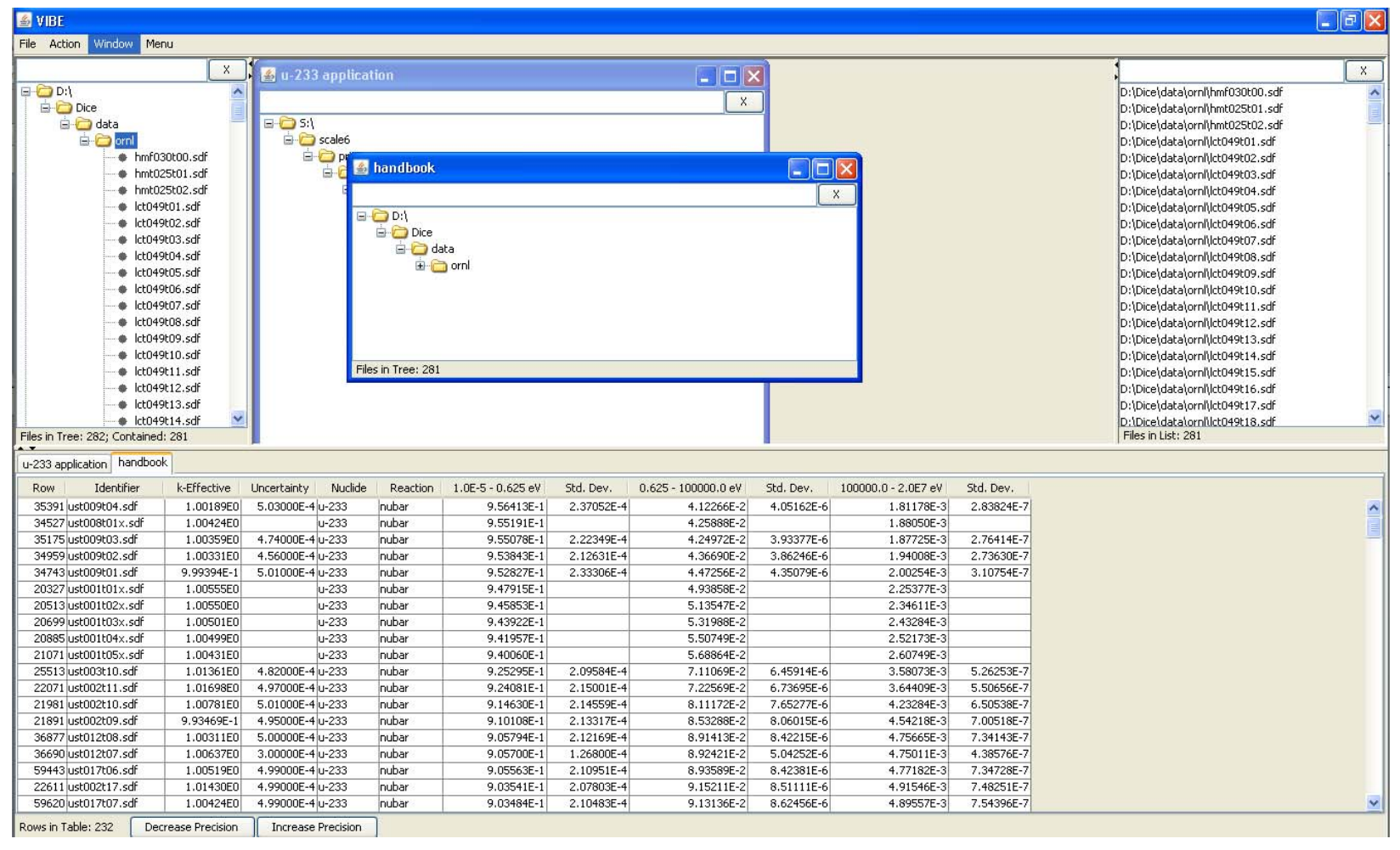

Fig. 74. VIBE with ${ }^{233} \mathrm{U}$ filter and descending sorting for thermal sensitivities applied to ICSBEP Handbook data. 


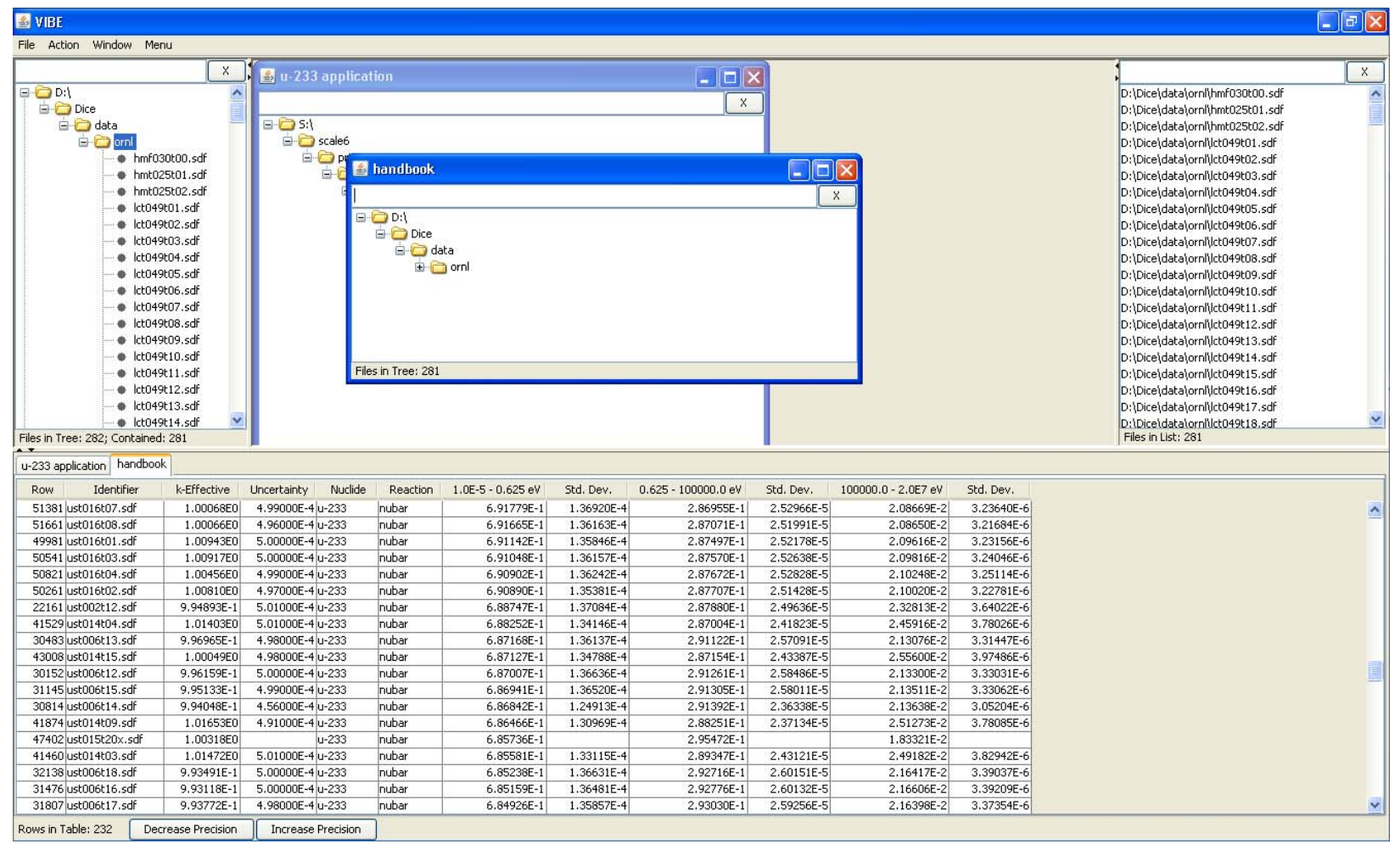

Fig. 75. Values near 0.69 from VIBE with ${ }^{233} \mathrm{U}$ filter and descending sorting for thermal sensitivities applied to ICSBEP Handbook data.

Returning to the filter dialog, available from the Action menu, add additional constraints to show only systems with ${ }^{233} \mathrm{U}$ nubar sensitivities with values between 0.67 and 0.71 , inclusive, as shown in Fig. 76. Once the filter is applied, the number of matching benchmarks is 33, as shown in Fig. 77.

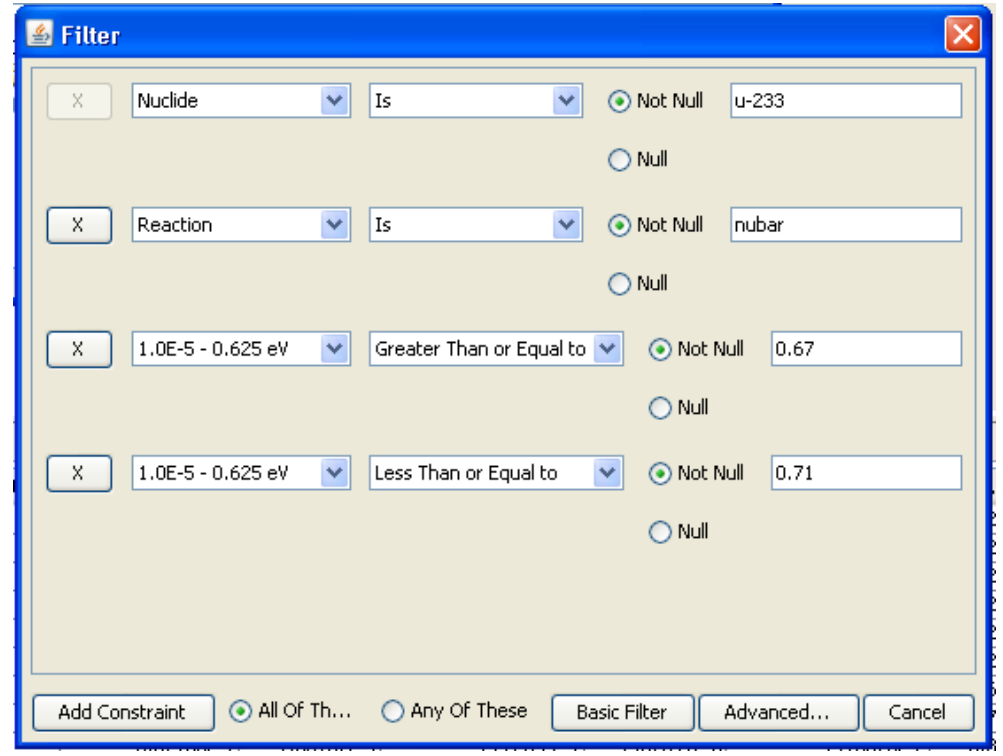

Fig. 76. VIBE Filter dialog for thermal ${ }^{233} U$ nubar sensitivities with values $\geq 0.67$ and $\leq 0.71$. 


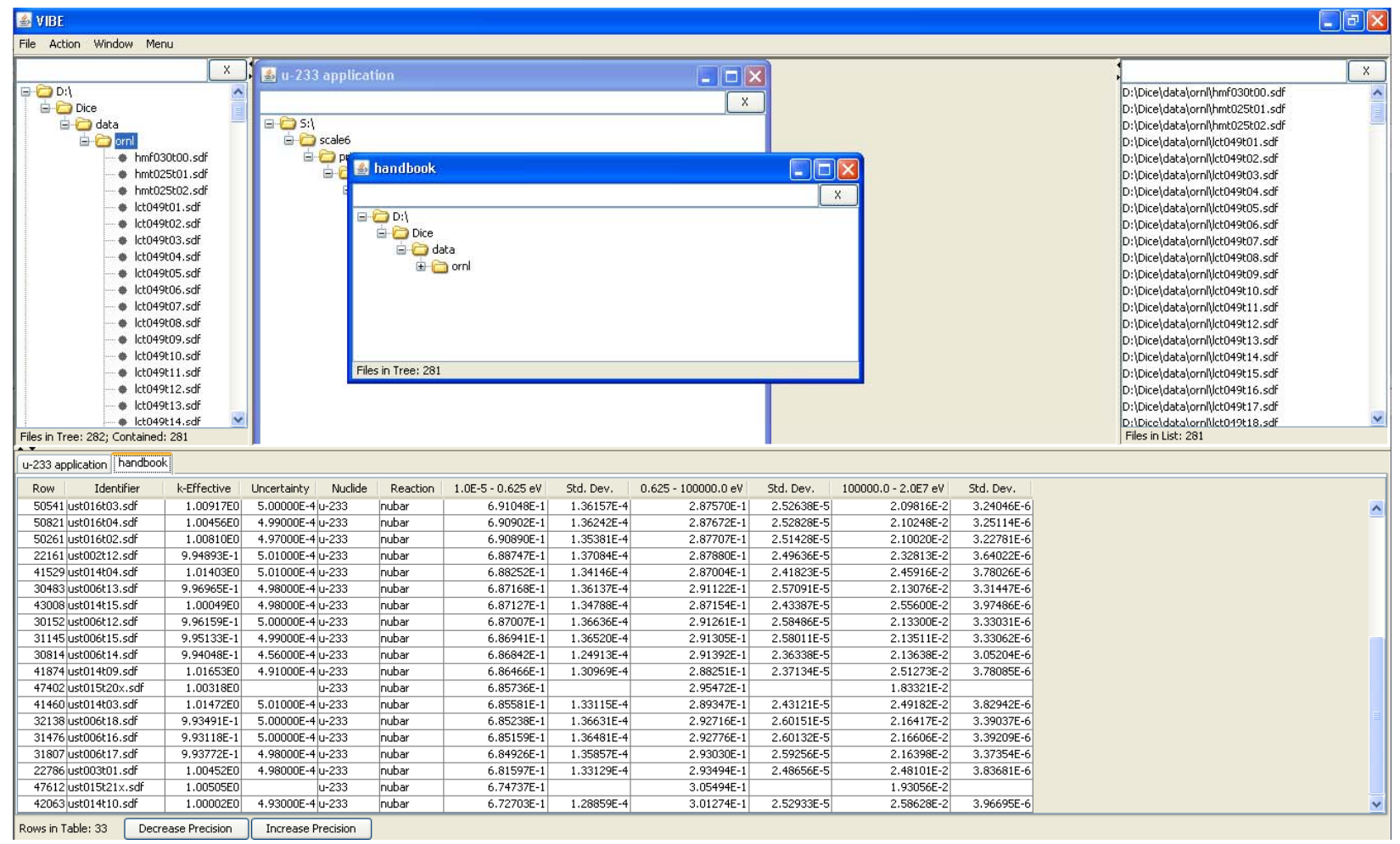

Fig. 77. VIBE with ${ }^{233} \mathrm{U}$ nubar thermal sensitivities near 0.69 filter applied to ICSBEP Handbook data.

The energy-dependent sensitivity data for the benchmarks can be visually compared to the sensitivities of the application using Javapeño. To open the benchmark SDF in Javapeño for the first benchmark shown in the table, U233-SOL-THERM-016-003, type ust016t03 in the filter field of the handbook benchmark set window in the center pane of VIBE, as shown in Fig. 78. Only the matching SDF will remain visible in the tree as long as the filter is applied. Right-click on the sdf file name and select Open in Javapeno. After the file is read from the handbook DVD, a new tab containing the sensitivity data will be added to the Javapeño reaction list, as shown in Fig. 79.

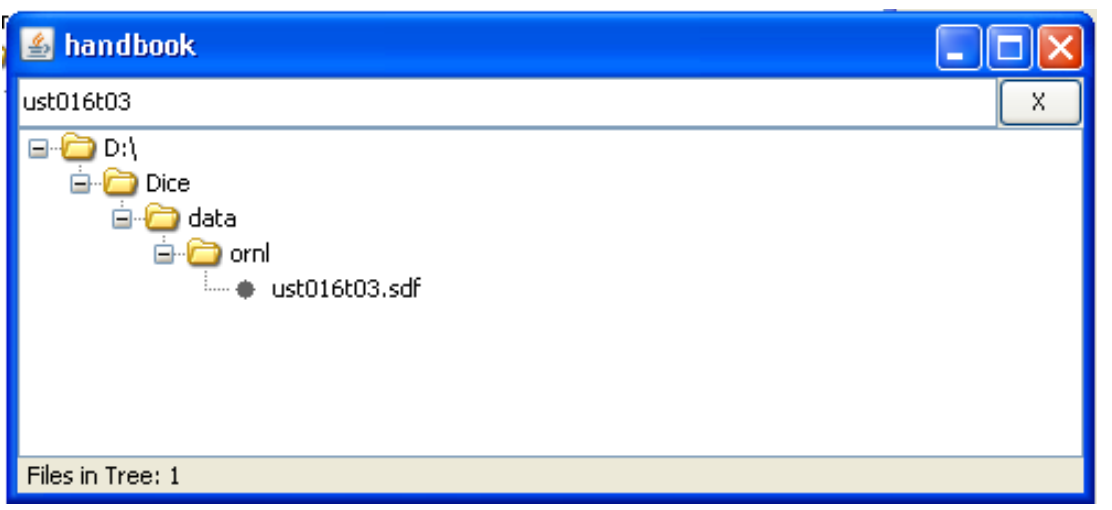

Fig. 78. Handbook benchmark set with filter applied. 


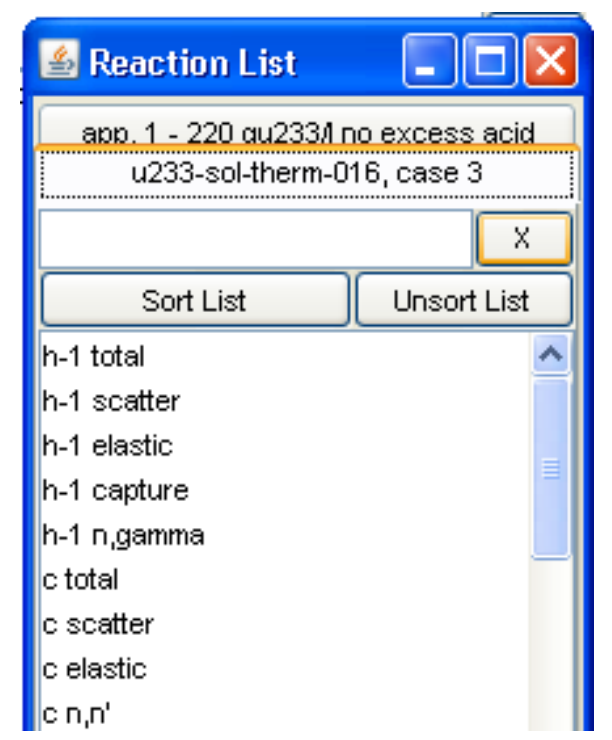

Fig. 79. Javapeño reaction list with data for ${ }^{233} \mathrm{U}$ application and U233-SOL-THERM-016-003.

First, plot the ${ }^{233} \mathrm{U}$ nubar sensitivity data for the application by selecting the application tab, scrolling to or filtering for u-233 nubar, right-clicking on u-233 nubar, and selecting Plot in new window from the pop-up menu, as shown in Fig. 80. Next, select the tab for the benchmark, filter for u-233 nubar, and double-click u-233 nubar in the list, as shown in Fig. 81, to add the benchmark sensitivity data to the plot of the application sensitivity data.

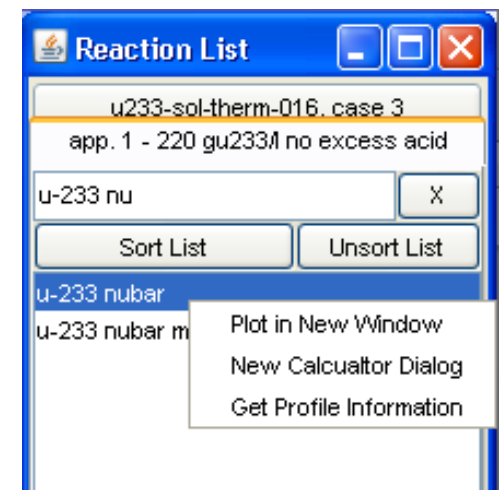

Fig. 80. Javapeño reaction list for plotting ${ }^{233} \mathrm{U}$ nubar sensitivity data for ${ }^{233} \mathrm{U}$ application in a new window. 


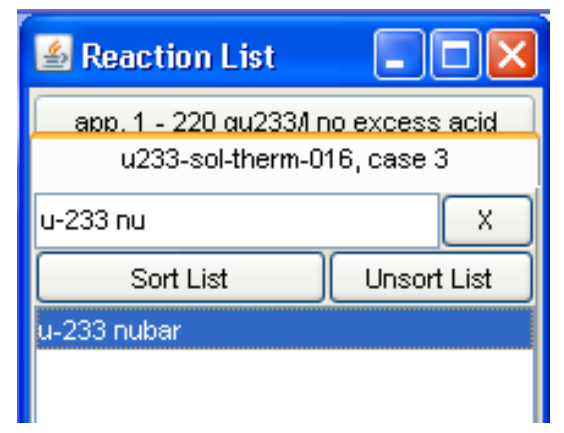

Fig. 81. Javapeño reaction list for plotting ${ }^{233} \mathrm{U}$ nubar sensitivity data for U233-SOL-THERM-016-003.

The resulting plot with the two sensitivity profiles is shown in Fig. 82. The two profiles are nearly identical across the entire energy spectrum, indicating that these two systems should have very similar bias in term of ${ }^{233} \mathrm{U}$ nubar. Other nuclides and reactions for this or other systems can be inspected in a similar manner.

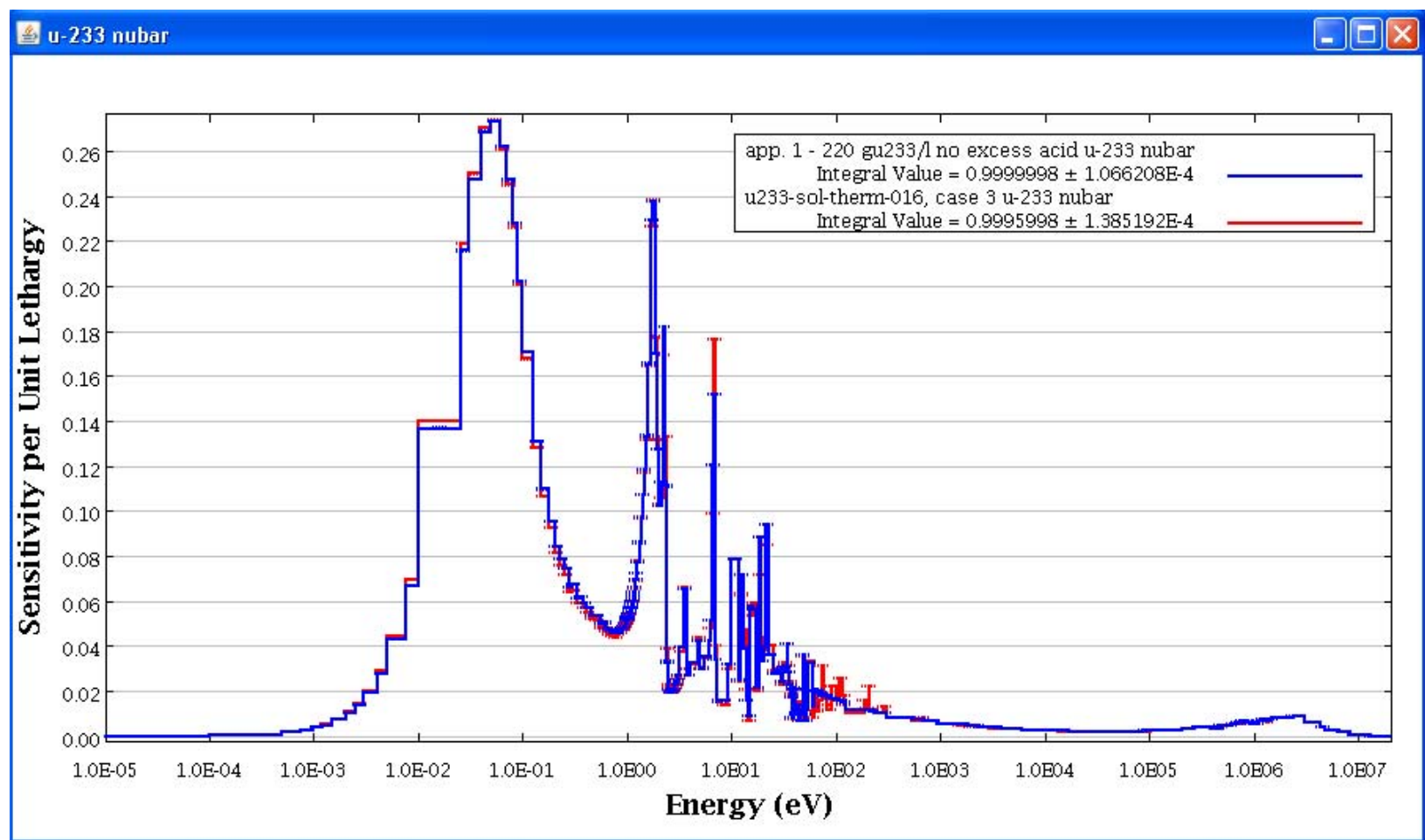

Fig. 82. Javapeño plot of ${ }^{233} U$ nubar sensitivities for ${ }^{233} U$ application and U233-SOL-THERM-016 case 3.

VIBE can also query the DICE database distributed with the ICSBEP Handbook. To access the database, the VIBE configuration must be set to identify the DICE database on the ICSBEP DVD. To update the configuration, select Configuration and then Update Configuration... from the VIBE File menu, as shown in Fig. 83 . 


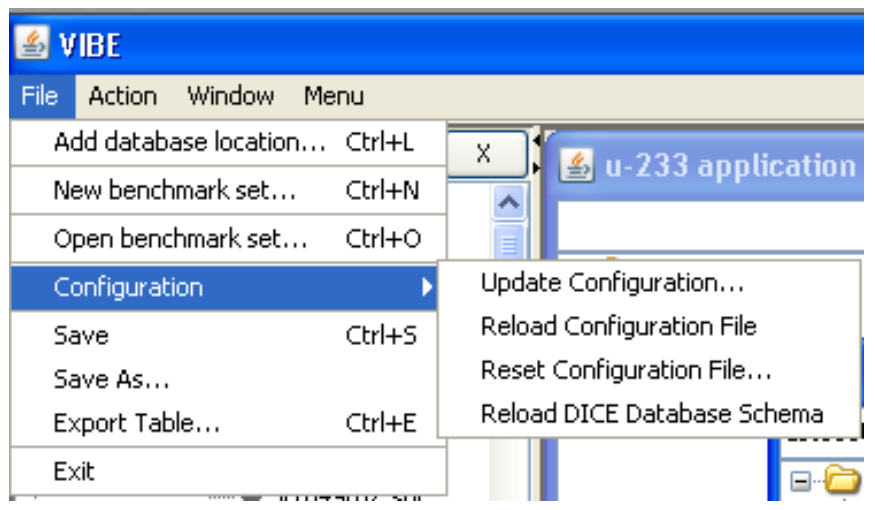

Fig. 83. VIBE configuration menu.

In the Configuration Manager dialog, click Browse... next to Database Location in the DICE section.

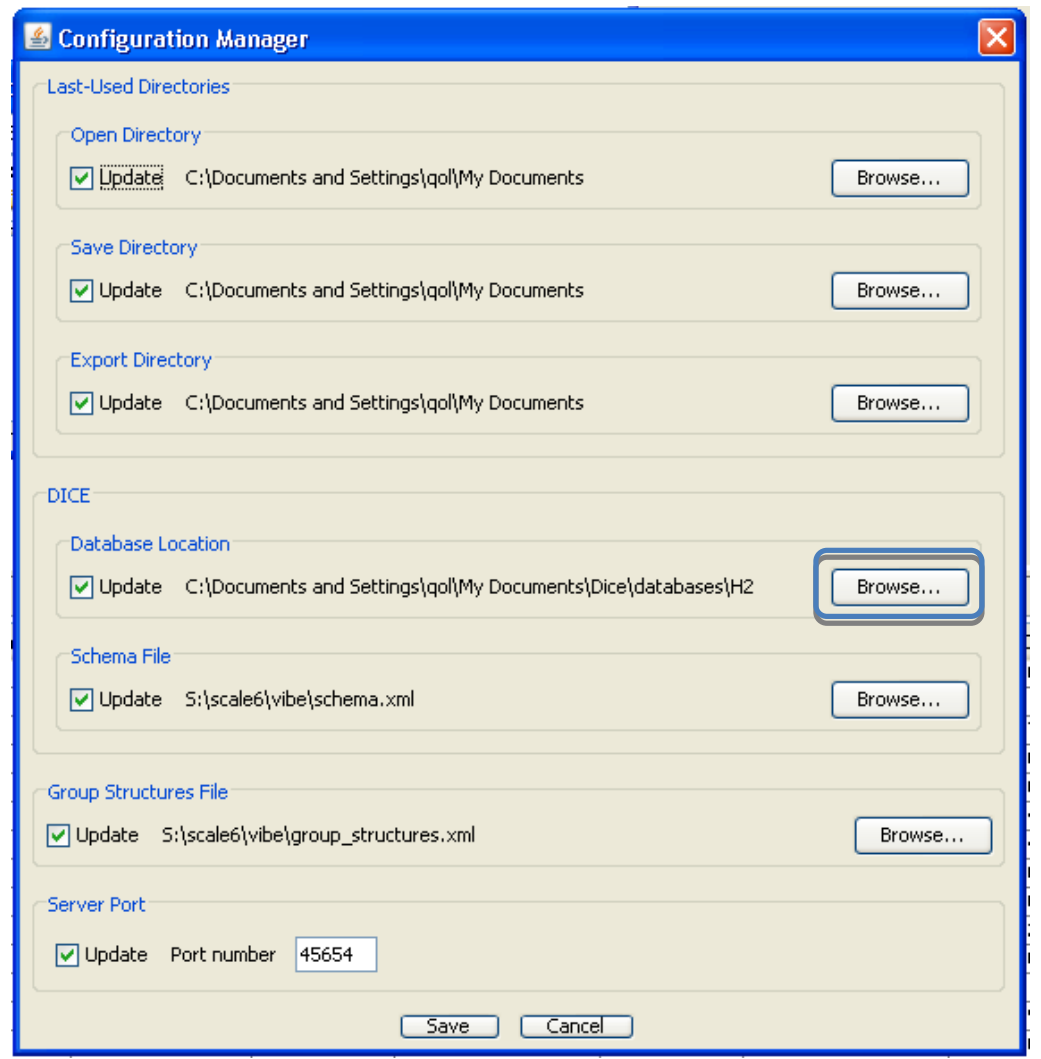

Fig. 84. VIBE configuration manager dialog.

In the file chooser dialog, browse to D: $\backslash$ Diceldatabases $\backslash H 2$ and click Accept, as shown in Fig. 85. 


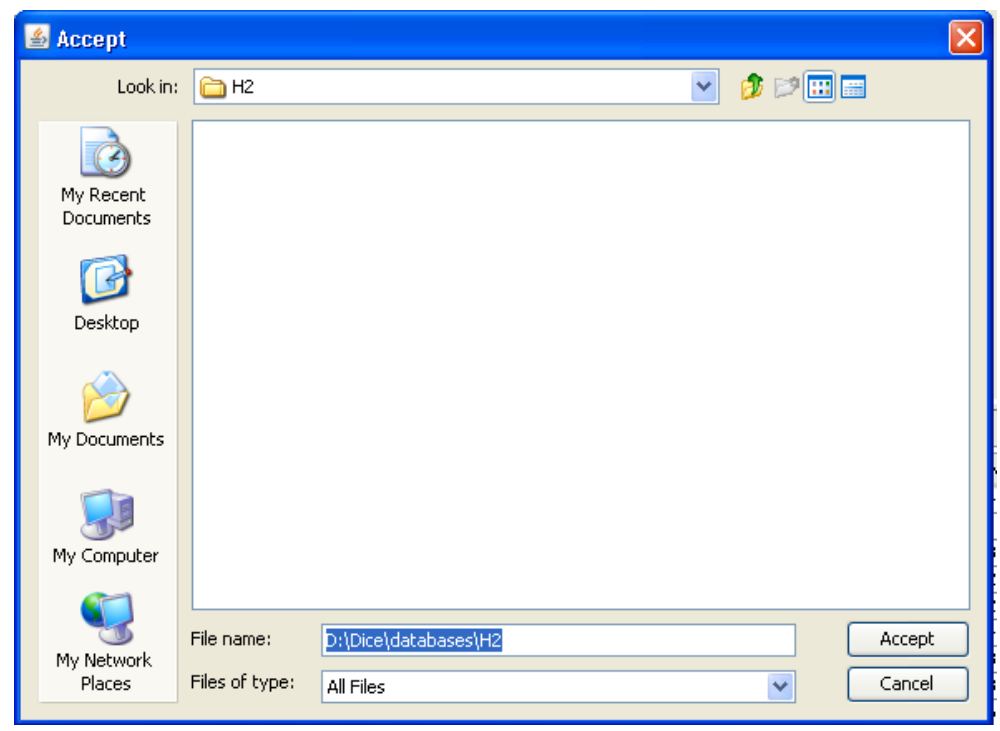

Fig. 85. File chooser to set location of DICE database.

Next, the DICE database is accessed to find specifications of ICSBEP benchmarks in a VIBE benchmark set. To determine details of the all experiments from U233-SOL-THERM-016, remove the 03 from the filter on the handbook benchmark set, as shown in Fig. 86. Note that there are 31 benchmarks in this evaluation. Checking the benchmarks from the table that have ${ }^{233} \mathrm{U}$ nubar thermal sensitivities near 0.69, only 11 of these 31 benchmarks from U233-SOL-THERM-016 meet the criteria.

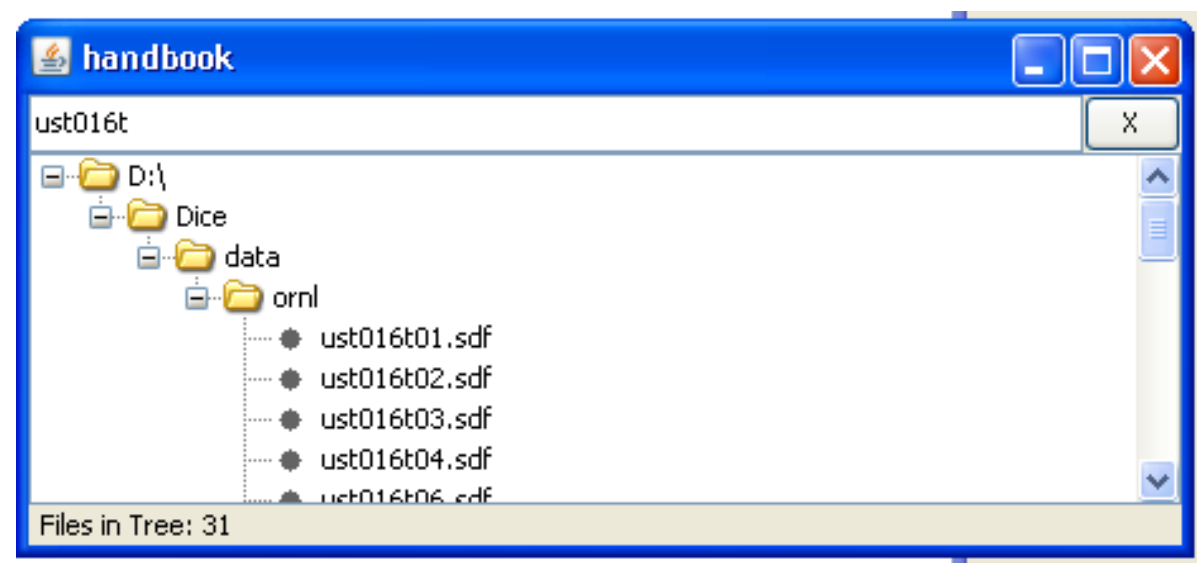

Fig. 86. Handbook benchmark set filtered for "ust016t."

To find details from the DICE database for all 31 benchmarks in U233-SOL-THERM-016, select Query DICE Database... from the Action menu. In the DICE query dialog, select Fuel from the left column, then check the boxes next to Fuel Concentration and Fuel Composition, as shown in Fig. 87. Under Benchmark Keff and Uncertainty, select Benchmark Keff and Benchmark Keff Uncertainty, as shown in Fig. 88, and then click View Data. 


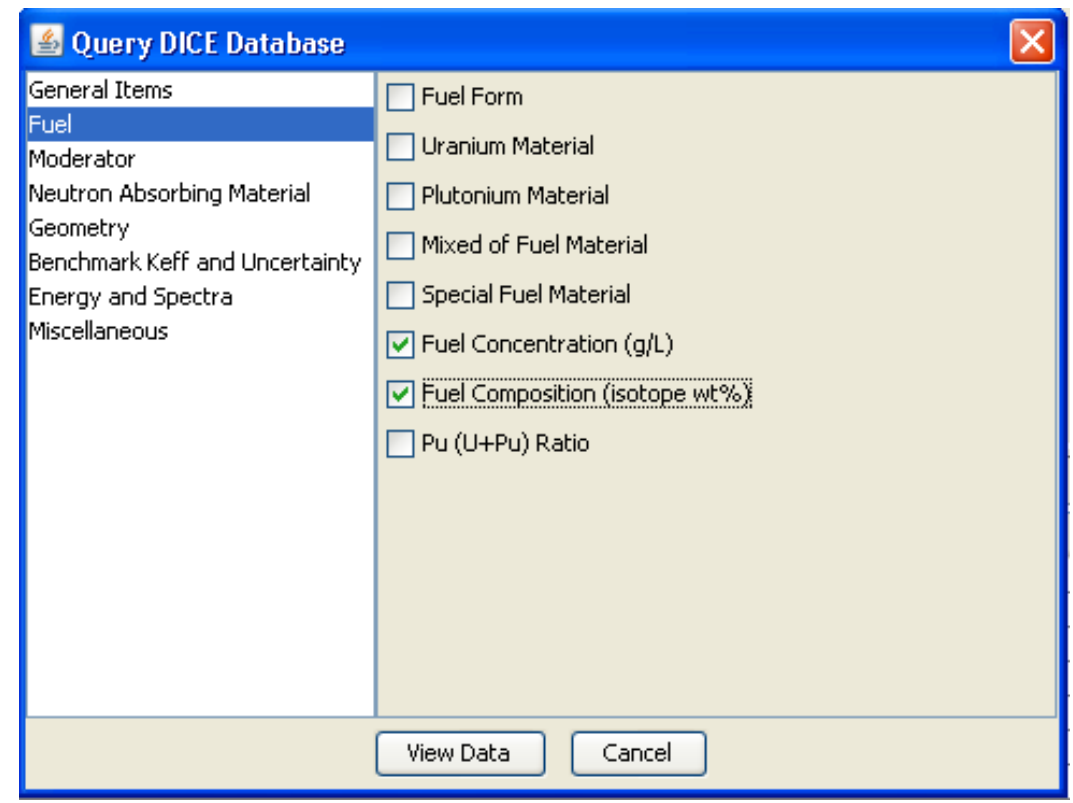

Fig. 87. Fuel options in DICE database query dialog.

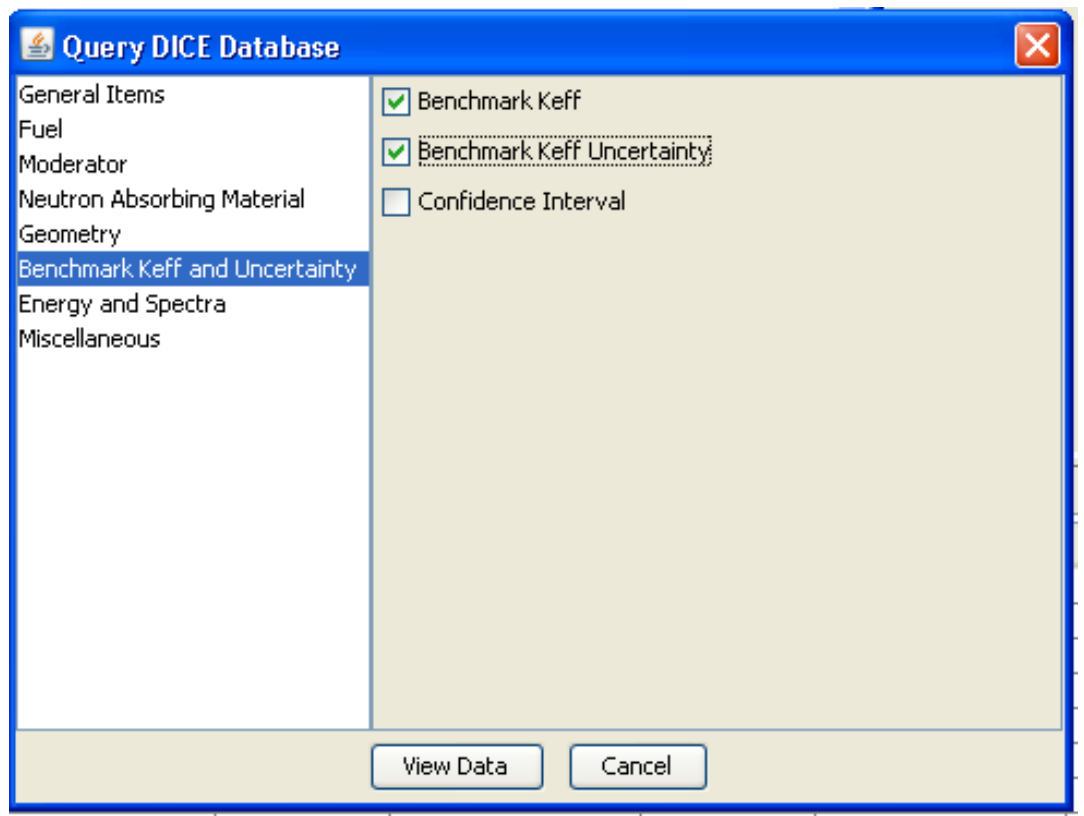

Fig. 88. Benchmark $k_{e f f}$ and uncertainty options in DICE database query dialog.

A new table containing the results of the DICE query will be added to VIBE, as shown in Fig. 89. Here it can be observed that these experiments have a $U$ content that is $97.5 \mathrm{wt} \%{ }^{233} \mathrm{U}$. Also, the solution concentration varies from 44.69 to $203.98 \mathrm{~g} / \mathrm{l}$. Recall that the application system was $100 \mathrm{wt} \%{ }^{233} \mathrm{U}$ and with a concentration of $200 \mathrm{~g} / \mathrm{l}$. Note that the 11 cases identified as having the ${ }^{233} \mathrm{U}$ nubar thermal sensitivities near that of the application are the cases with solution concentrations of $200 \mathrm{~g} / \mathrm{l}$. The selection of the same 11 experiments based on TSUNAMI sensitivity criteria or solution concentration criteria confirms the validity of the TSUNAMI approach in comparison to more traditional approaches. 
Using VIBE with a DICE query and filter applied to the resulting table to identify U233-SOL-THERM experiments with solution concentrations between 180 and $220 \mathrm{~g} / \mathrm{l}$ reveals 29 matching cases, as shown in Fig. 90. Here U233-SOL-THERM-002 case 1 is shown to have a solution concentration of $200.45 \mathrm{~g} / 1$. However, TSUNAMI data showed matching ${ }^{233} \mathrm{U}$ nubar sensitivity for U233-SOL-THERM-002 case 12 , not case 1. Using another DICE query to examine all cases from U233-SOL-THERM-002, shown in Fig. 91, reveals that case 12 has a concentration of $277.585 \mathrm{~g} / 1$.

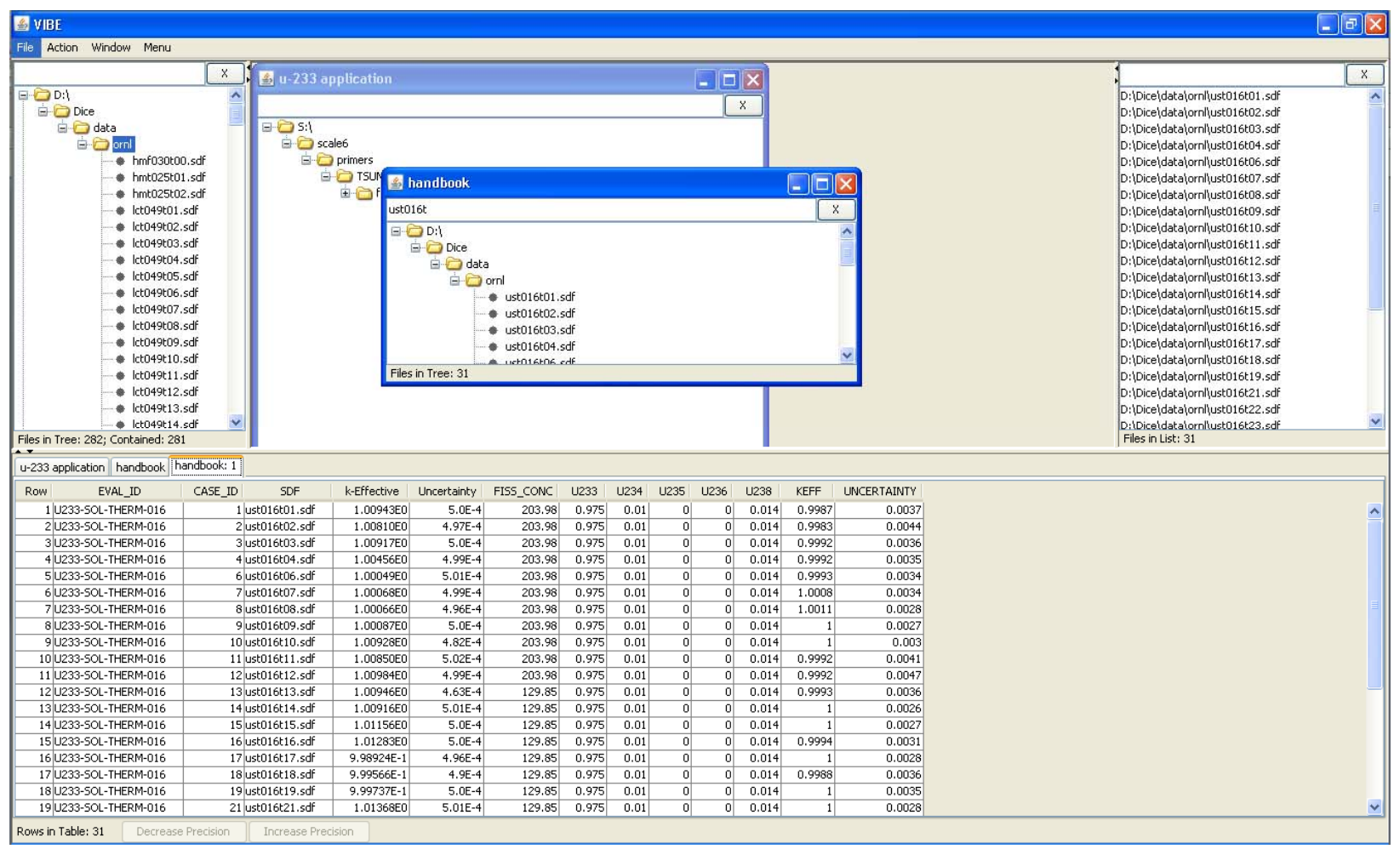

Fig. 89. VIBE with table of DICE query results. 


\begin{tabular}{|c|c|c|c|c|}
\hline 1-233 application handbook & andbook: 1 handbook: 2 , & handbook: 3 & & \\
\hline EVAL_ID & CASE_ID & $k$-Effective & Uncertainty & FISS_CONC \\
\hline 105 U233-SOL-THERM-002 & 1 ust002t01.sdf & $1.00750 \mathrm{EO}$ & $4.99 \mathrm{E}-4$ & 200.45 \\
\hline 132 U233-SOL-THERM-004 & 1 ust004t01.sd & $1.00461 \mathrm{E0}$ & $5.02 \mathrm{E}-4$ & 200.453 \\
\hline 153 U233-SOL-THERM-006 & 12 ust006t12.sd & $9.96159 \mathrm{E}-1$ & $5.0 \mathrm{E}-4$ & 203.8 \\
\hline 154 U233-SOL-THERM-006 & 13 ust006t13.sd & $9.96965 \mathrm{E}-1$ & $4.98 \mathrm{E}-4$ & 203.8 \\
\hline 155 U233-SOL-THERM-006 & 14 ust006t14.sd & $9.94048 \mathrm{E}-1$ & $4.56 \mathrm{E}-4$ & 203.8 \\
\hline 156 U233-SOL-THERM-006 & 15 ust006t15.sd & $9.95133 \mathrm{E}-1$ & $4.99 \mathrm{E}-4$ & 203.8 \\
\hline 157 U233-SOL-THERM-006 & 16 ust006t16.sd & $9.93118 \mathrm{E}-1$ & $5.0 \mathrm{E}-4$ & 203.8 \\
\hline 158 U233-SOL-THERM-006 & 17 ust006t17.sd & $9.93772 \mathrm{E}-1$ & $4.98 \mathrm{E}-4$ & 203.8 \\
\hline 159 U233-SOL-THERM-006 & 18 ust006t18.sd & $9.93491 \mathrm{E}-1$ & $5.0 \mathrm{E}-4$ & 203.8 \\
\hline 160 U233-SOL-THERM-006 & 19 ust006t19.sd & $9.99731 \mathrm{E}-1$ & $4.99 \mathrm{E}-4$ & 203.8 \\
\hline 161 U233-SOL-THERM-006 & 20 ust006t20.sd & $9.98818 \mathrm{E}-1$ & $5.01 \mathrm{E}-4$ & 203.8 \\
\hline 162 U233-SOL-THERM-006 & 21 ust006t21.sdf & $9.97874 \mathrm{E}-1$ & $4.98 \mathrm{E}-4$ & 203.8 \\
\hline 163 U233-SOL-THERM-006 & 22 ust006t22.sdf & $9.98336 \mathrm{E}-1$ & $5.0 \mathrm{E}-4$ & 203.8 \\
\hline 164 U233-SOL-THERM-006 & 23 ust006t23.sdf & $9.98317 \mathrm{E}-1$ & $4.99 \mathrm{E}-4$ & 203.8 \\
\hline 165 U233-SOL-THERM-006 & 24 ust006t24.sdf & $9.98268 \mathrm{E}-1$ & $4.98 \mathrm{E}-4$ & 203.8 \\
\hline 166 U233-SOL-THERM-006 & 25 ust006t $25 . s \mathrm{~d}$ & $1.00019 \mathrm{E} 0$ & $5.0 \mathrm{E}-4$ & 203.8 \\
\hline 173 U233-SOL-THERM-012 & 1 ust012t01.sd & $1.00188 \mathrm{E} 0$ & $4.85 \mathrm{E}-4$ & 200.2 \\
\hline 174 U233-SOL-THERM-012 & 2 ust012t02.sd & $1.00180 \mathrm{E} 0$ & $4.98 \mathrm{E}-4$ & 190.4 \\
\hline 244 U233-SOL-THERM-016 & 1 ust016t01.sd & $1.00943 \mathrm{EO}$ & $5.0 \mathrm{E}-4$ & 203.98 \\
\hline
\end{tabular}

Fig. 90. VIBE DICE query results for all U233-SOL-THERM experiments filtered from concentrations near $200 \mathrm{~g} / \mathrm{l}$.

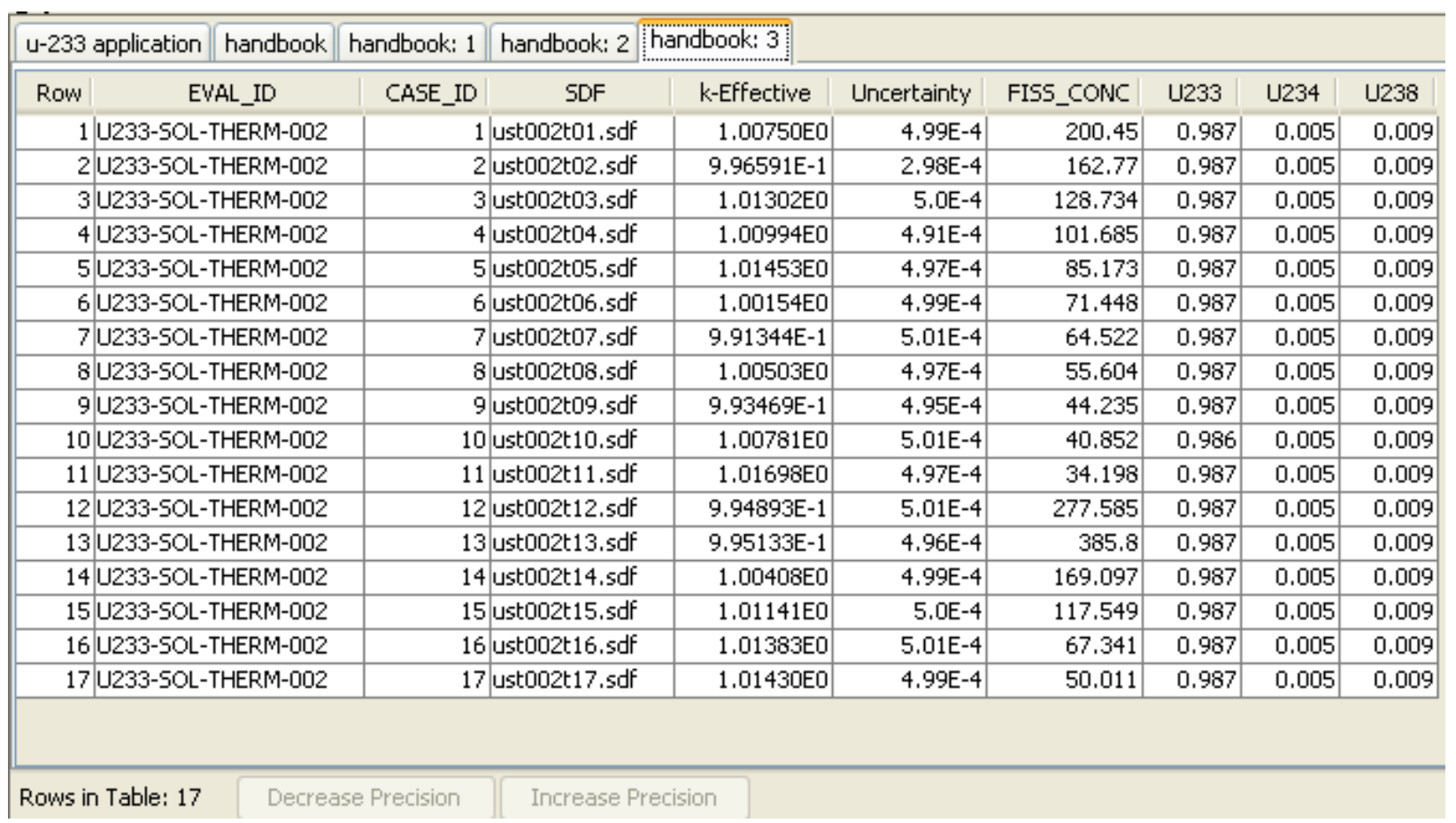

Fig. 91. VIBE DICE query results for U233-SOL-THERM-002 benchmarks. 
Using VIBE to open the SDFs in Javapeño and then plotting ${ }^{233} \mathrm{U}$ nubar for the two benchmarks and the application reveals a higher sensitivity to ${ }^{233} U$ nubar for case 1 than for case 12 , which more closely matches the sensitivity of the application. In this case the moderation of the solutions is different enough to shift the spectra, causing the case with the higher solution concentration to more closely match the application. In this instance, a thorough and potentially time-consuming review of data from the handbook evaluation or the DICE database could have revealed these subtleties to an expert, where the TSUNAMI sensitivity data quickly reveal similarity in terms of the physics of the systems, not just the characteristics of the compositions.

\section{u-233 nubar}

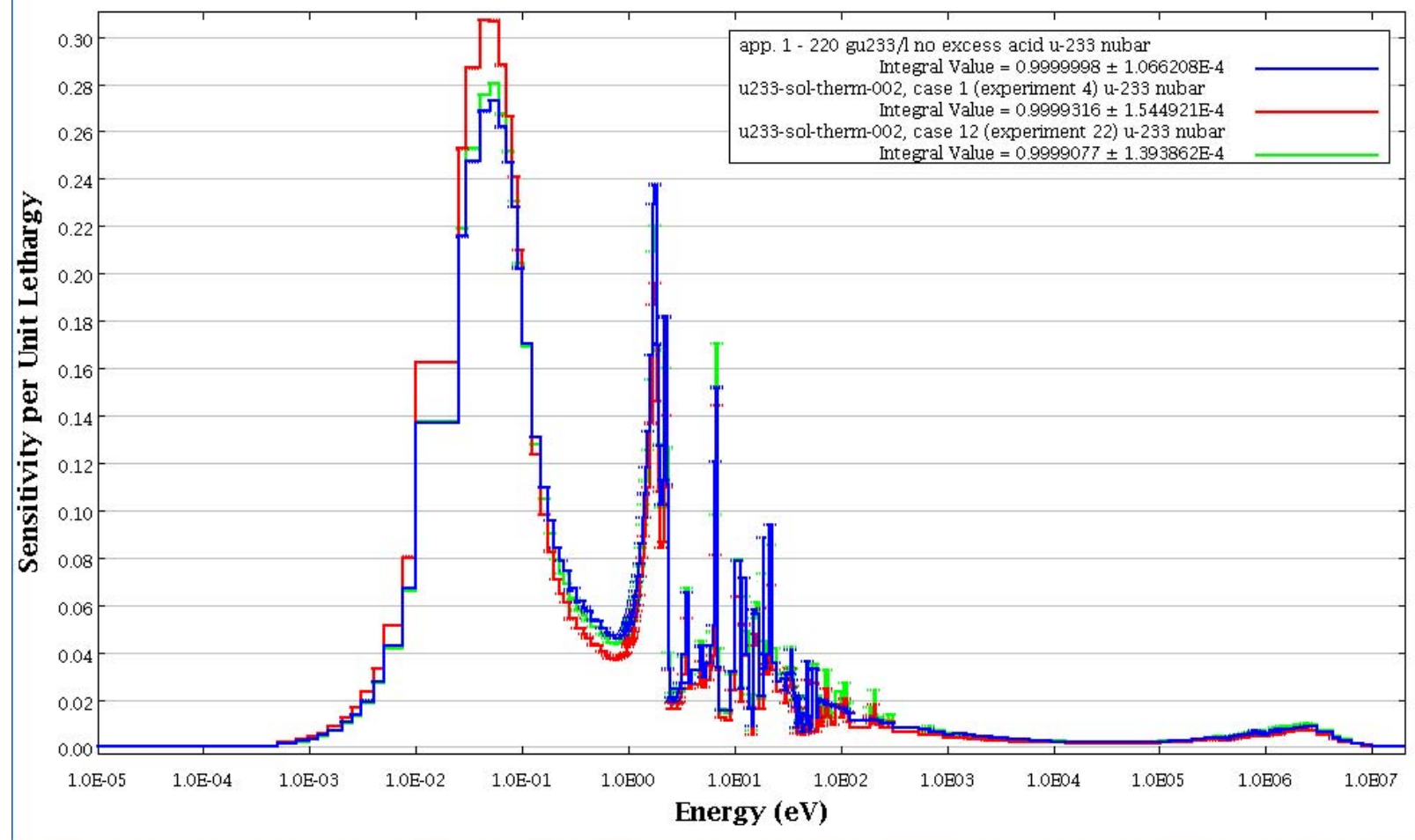

Fig. 92. Sensitivity profiles for ${ }^{233} U$ nubar from ${ }^{233} U$ application and U233-SOL-THERM-002 cases 1 and 12.

A final step in the use of VIBE is to export the desired files in a format suitable for more advanced analysis with TSUNAMI-IP (TSUNAMI Indices and Parameters) and TSURFER (Tool for Sensitivity

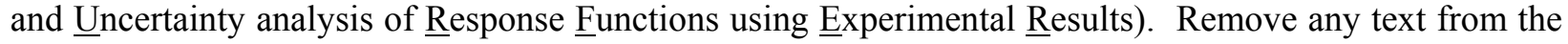
filter field of the handbook window in the center benchmark pane of VIBE so that all 281 benchmarks are listed. Select Save AS... from the File menu. In this example, a new folder on C: drive called tsunami is created. Browse to the destination directory, ensure that the file type is set to VIBE Benchmark Sets (.vdf), and click Save. A new file called handbook. vdf will be saved to provide easy access to the benchmarks in the ExSITE interface. 


\subsection{BUILDING A TSUNAMI-IP INPUT WITH EXSITE}

The TSUNAMI-IP code uses sensitivity data from benchmark experiments and target applications along with the cross-section-covariance data to numerically quantify the similarity of a benchmark to a target

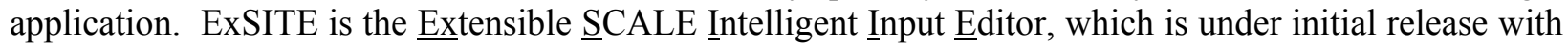
SCALE 6 to assist with TSUNAMI analyses using TSUNAMI-IP, TSURFER, and TSAR (Tool for Sensitivity Analysis of Reactivity Responses). At the time of this writing, ExSITE is still in development, so the version released with SCALE 6 may be slightly different than that shown here.

To begin, double-click the ExSITE icon in the SCALE 6 shortcut menu. ExSITE is based on the NetBeans development platform for Java, so the initial startup page provides some information on NetBeans. To close this page, simple click the $x$ on the Start Page tab. In future updates, this start page will provide information on SCALE.

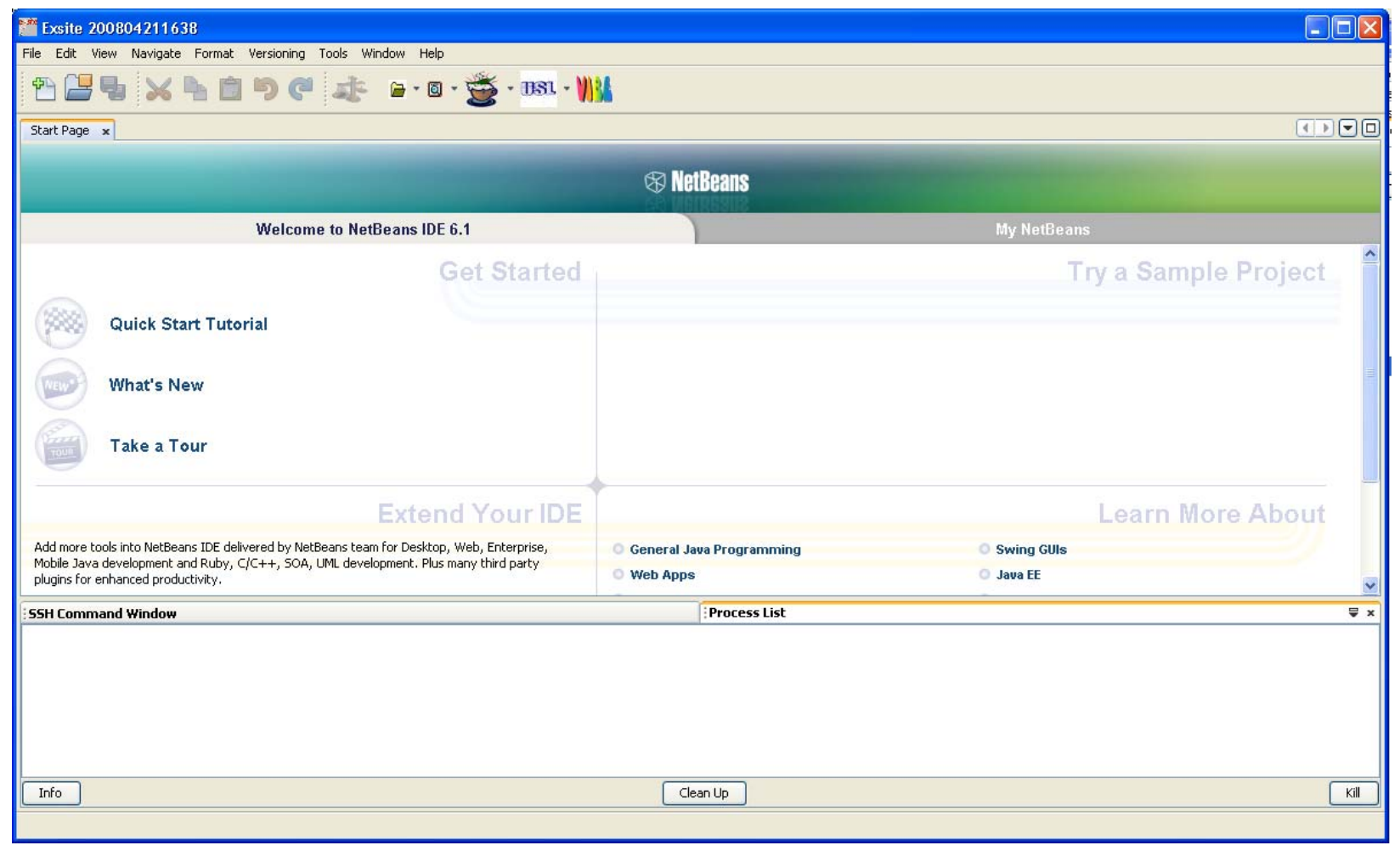

Fig. 93. ExSITE welcome page.

To begin a new input file, click the icon with the green + at the top left of the toolbar. In the input file wizard, shown in Fig. 94, click Choose File and Location. In the file chooser, browse to the directory previously created for this example, $C: \backslash t$ sunami, then enter tsunami-ip.inp as the file name and click Save. In the input file wizard, click Finish. ExSITE will load a new blank input file named tsunami - ip. inp and open the palette for building input files on the right side of the screen, as shown in Fig. 96. 


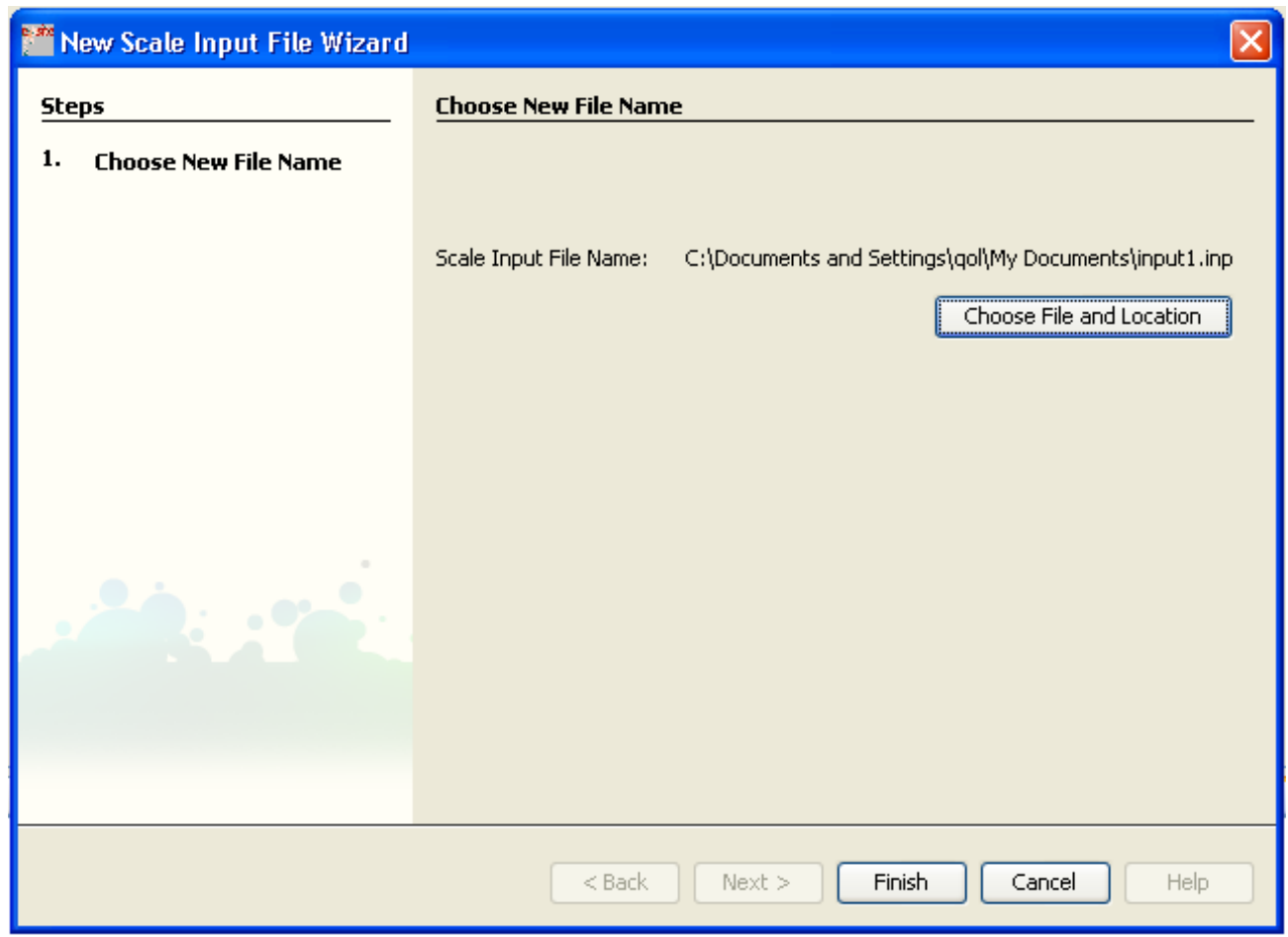

Fig. 94. ExSITE new input file wizard.

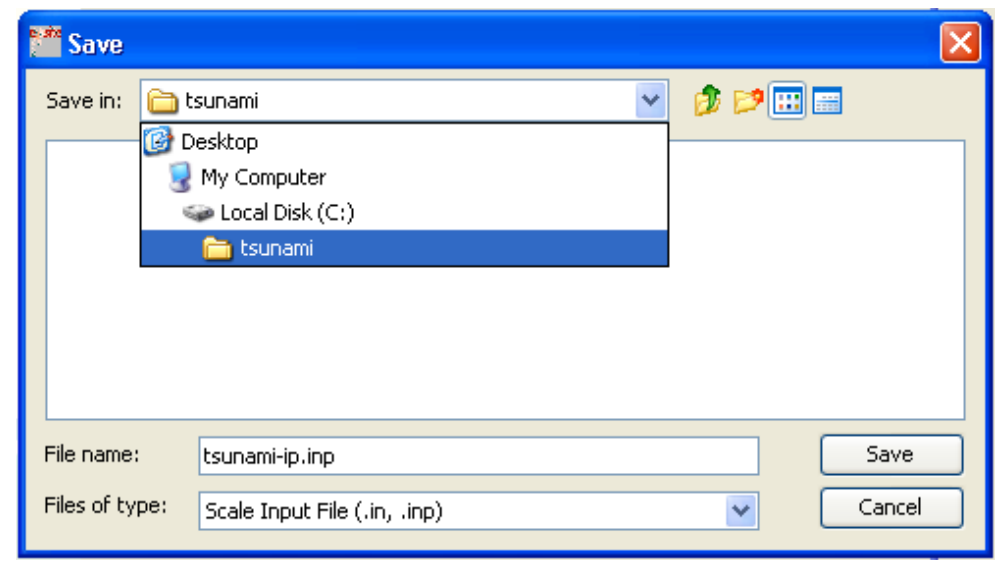

Fig. 95. ExSITE file chooser for TSUNAMI-IP input file. 


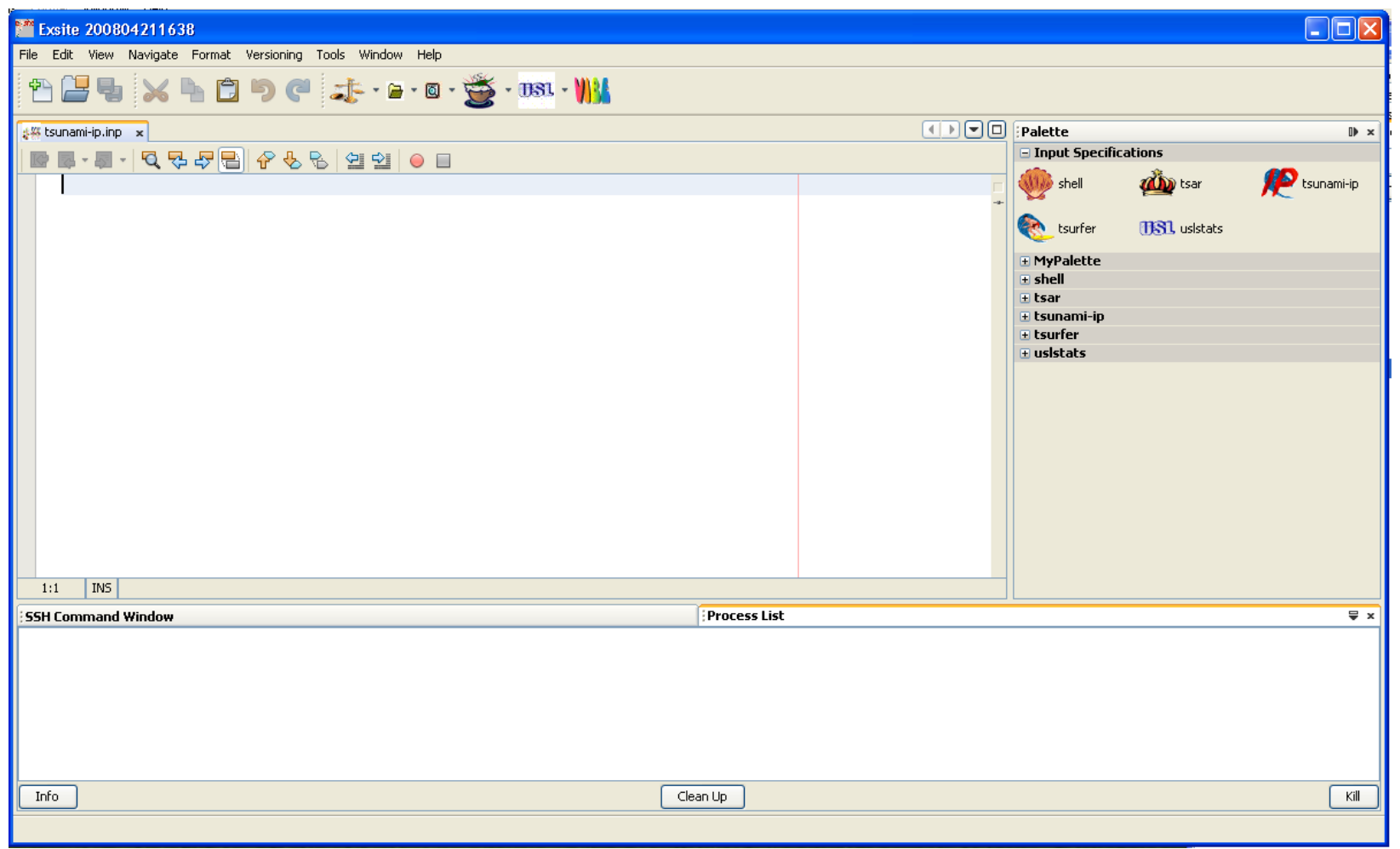

Fig. 96. ExSITE with empty input file.

The TSUNAMI-IP input file can be built by typing directly in the input file or by double-clicking the TSUNAMI-IP icon in the palette. In this example, the TSUNAMI-IP palette will primarily be used. Double-click the TSUNAMI-IP icon in the palette, and the dialog for TSUNAMI-IP input will appear. Check the box next to title, to indicate that this input will use an optional title, and enter $u-233$ application, as shown in Fig. 97. The input blocks for parameter, experiments, and applications are required. Note that the checkboxes next to each one cannot be turned off. There are tabs at the top of the dialog for each of these input blocks. Optional blocks for reactions, covariance, exclusions, and HTML can be enabled as needed by checking the box next to each one. For each box that is checked, a new tab will appear at the top of the input dialog. 


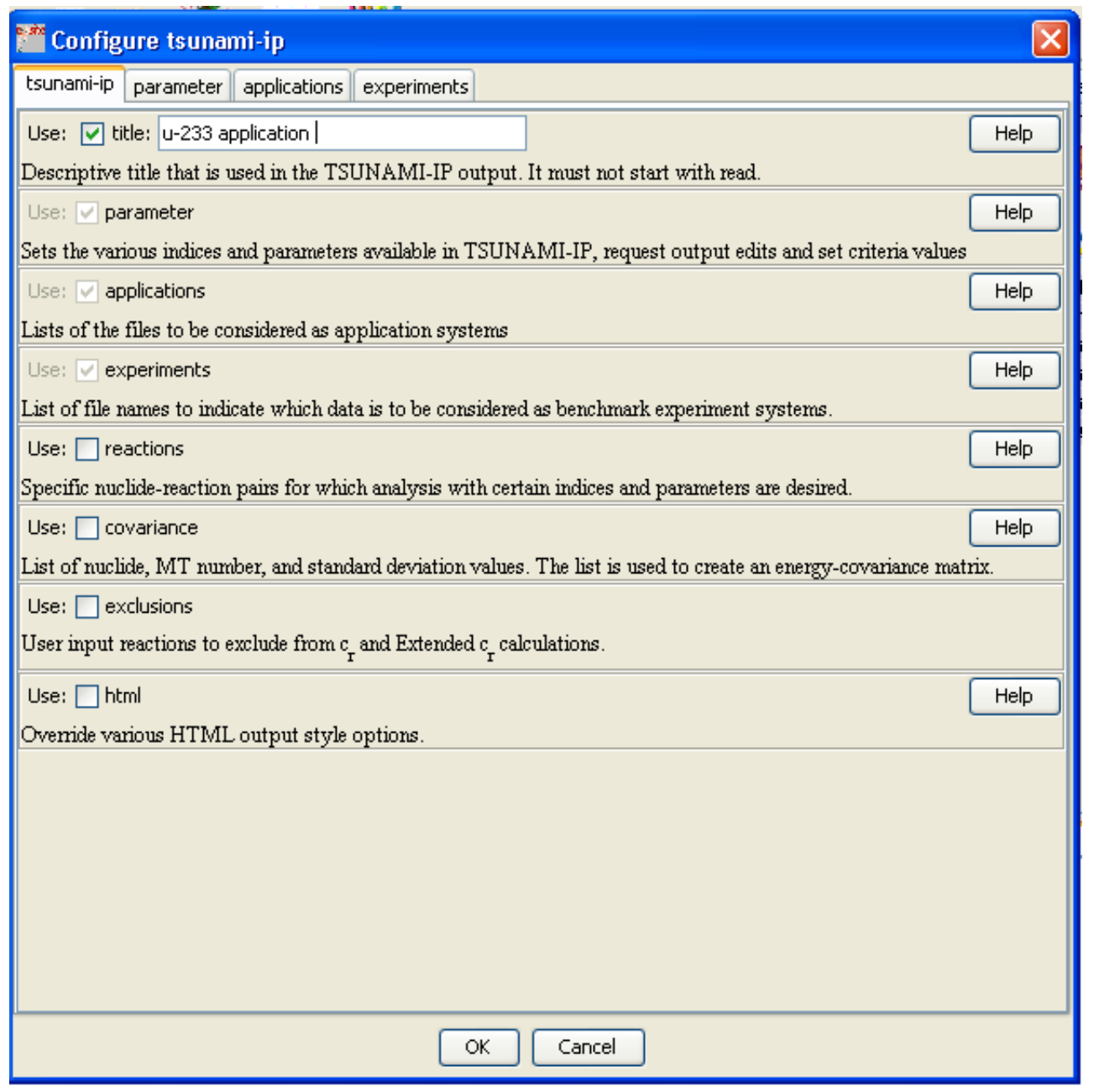

Fig. 97. ExSITE TSUNAMI-IP palette dialog.

\subsubsection{Applications}

Skipping the parameter data for now, click on the applications tab to add the ${ }^{233} \mathrm{U}$ application to the TSUNAMI-IP analysis. TSUNAMI-IP identifies applications with the path to the SDF for the desired system. In the applications tab, click Add Applications to enable a field in which a new application is identified. Click Choose a File and browse to the location of the SDF for the ${ }^{233} \mathrm{U}$

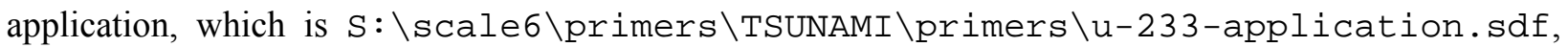
as shown in Fig. 98. Note that in this example, SCALE 6 is installed on the S: drive. In general SCALE 6 is most likely installed on the C: drive. Once the SDF is located, click OK to close the TSUNAMI-IP input dialog and add initial data to the TSUNAMI-IP input file. The text of the TSUNAMI-IP input file will appear in ExSITE as shown in Fig. 99. 


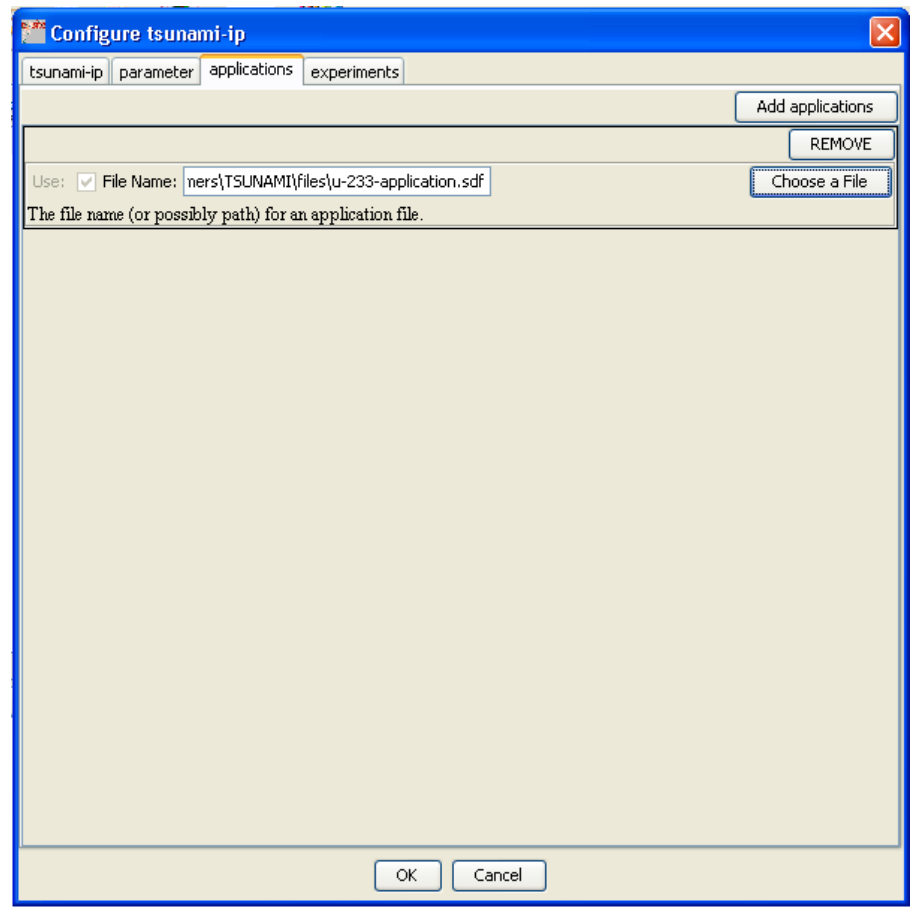

Fig. 98. ExSITE applications tab for TSUNAMI-IP input with ${ }^{233} \mathrm{U}$ application.

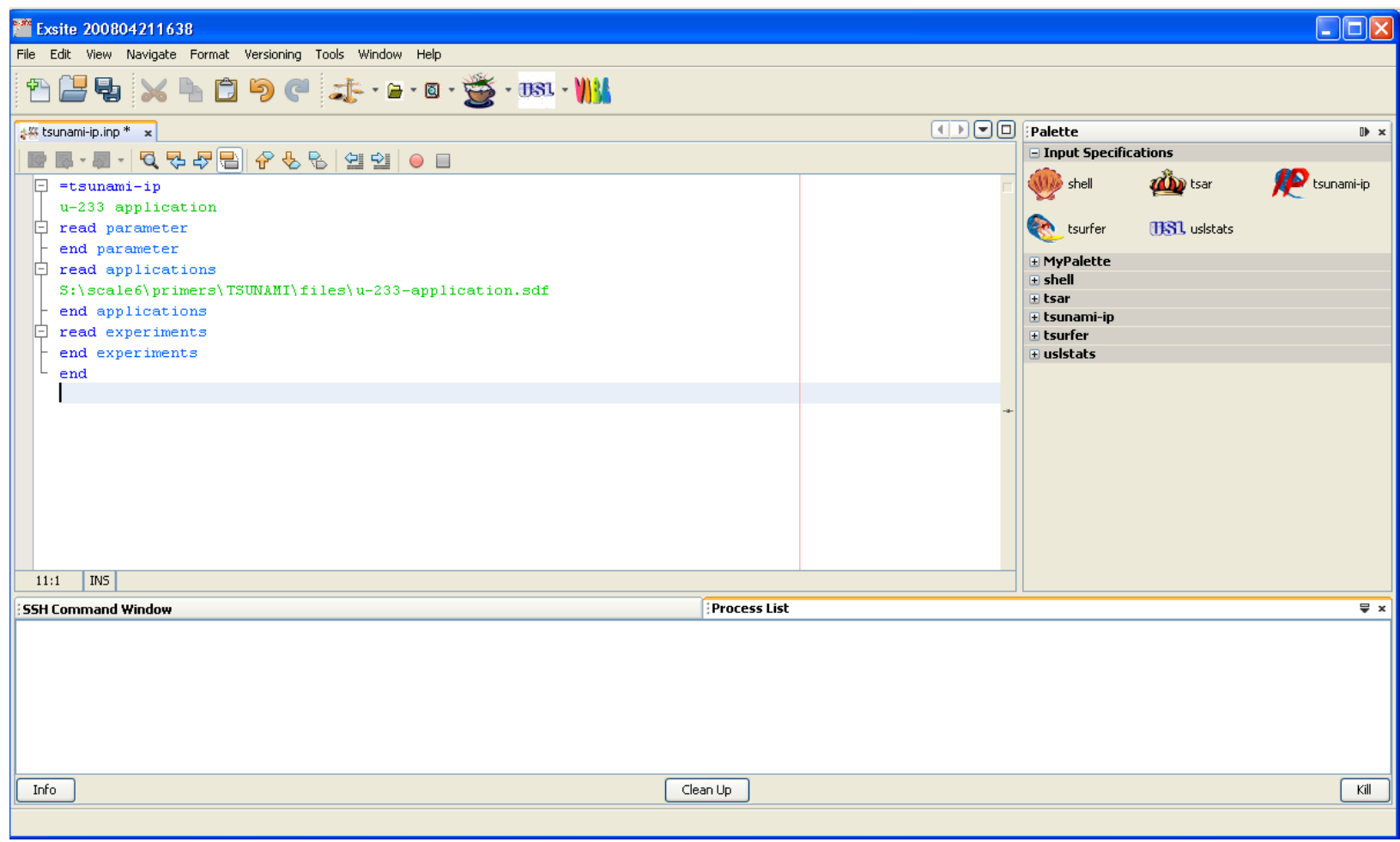

Fig. 99. ExSITE with ${ }^{233} \mathrm{U}$ application identified in TSUNAMI-IP input. 
Each of the 281 SDFs for the benchmarks also needs to be entered in the TSUNAMI-IP experiments block. However, use of the ExSITE dialog to browse to each file would be extremely time consuming. Recall that a final step in the use of VIBE was to save the benchmark set in the VIBE data file (VDF) format. ExSITE provides the ability to import a VDF for use in TSUNAMI-IP. In the ExSITE File menu, shown in Fig. 100, select Open File... In the resulting file chooser, browse to the location of the file handbook.vdf, which in this example is the same as the location of the TSUNAMI-IP input file, C:Itsunami. Set the file type to VIBE Data File (.vdf), as shown in Fig. 101, select the file handbook. vdf, and click Open.

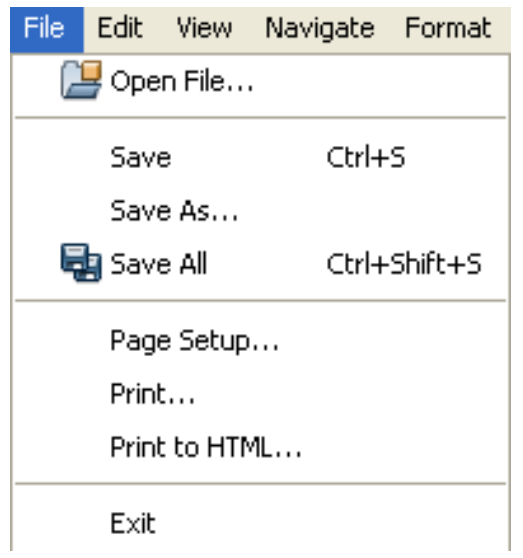

Fig. 100. ExSITE file menu.

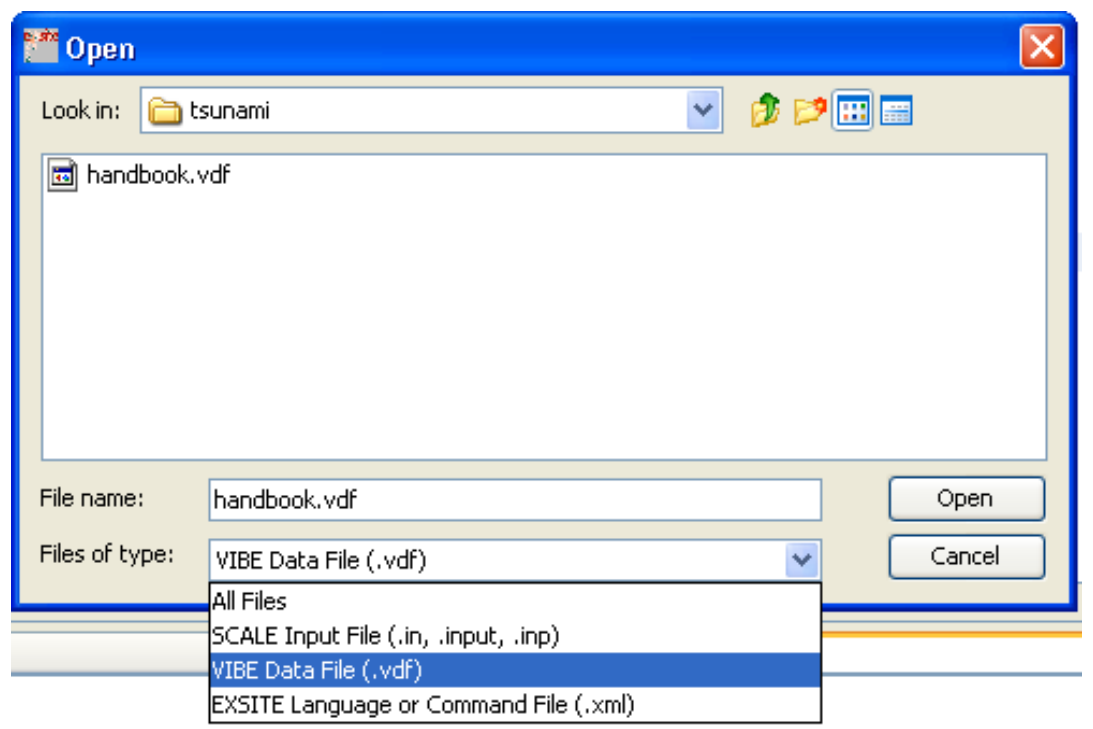

Fig. 101. ExSITE file chooser to load handbook.vdf.

The VDF will load under MyPalette on the right side of ExSITE. Clicking the + sign next to MyPalette will cause the palette section to open, revealing the icon for the handbook data. To add all 281 experiments to the TSUNAMI-IP input, click in the input file at the end of the read 
experiments line, then double-click the handbook icon under MyPalette. Alternatively, the handbook data can be added to the input file by dragging and dropping the handbook icon to the end of the read experiments line in the input file.

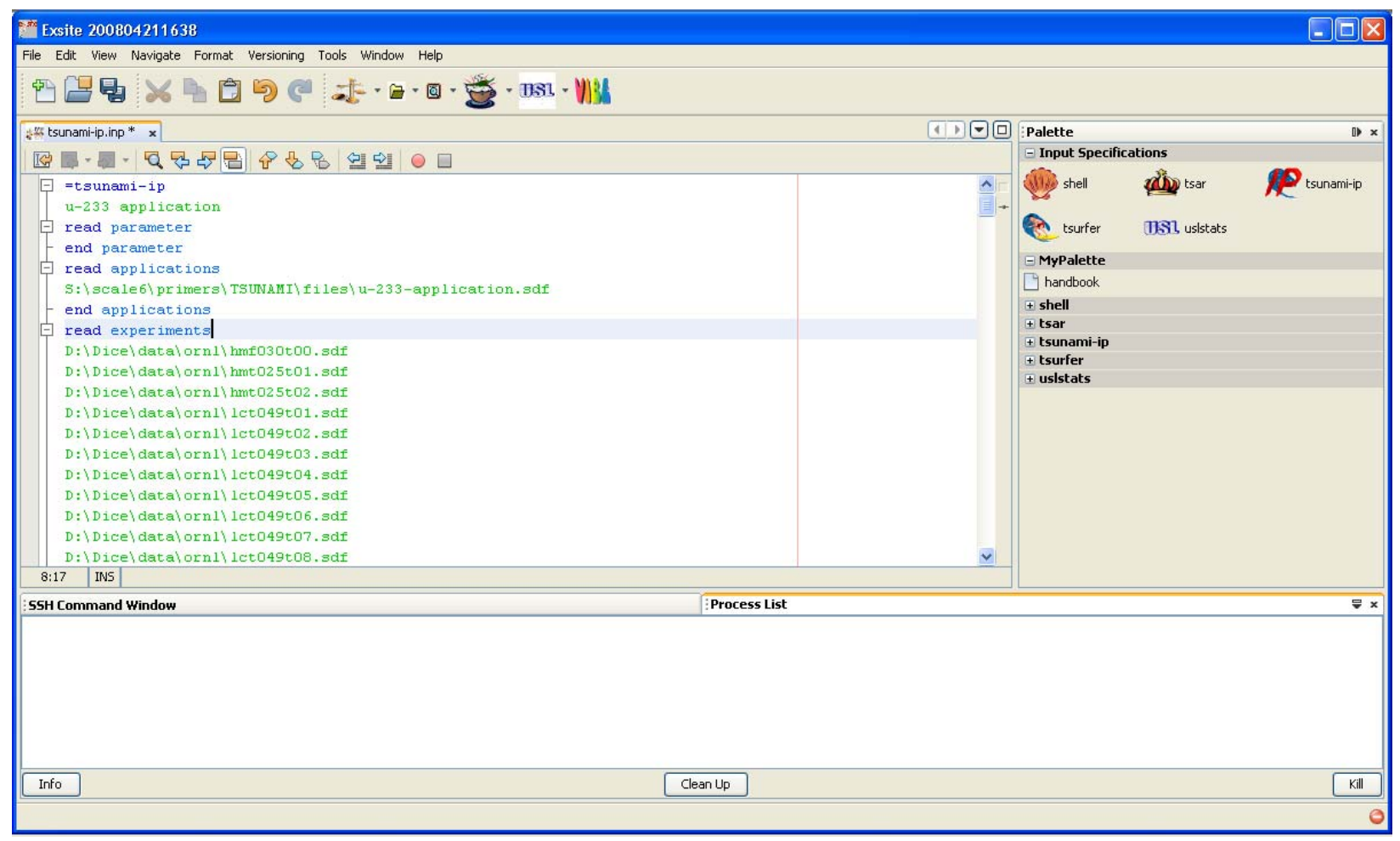

Fig. 102. ExSITE with handbook data added to the palette and to the read experiments input block.

When the TSUNAMI-IP dialog is opened again by double-clicking on the TSUNAMI-IP icon in the palette, the experiments tab will be populated with the 281 SDFs loaded by the VDF, as shown in Fig. 103 . 


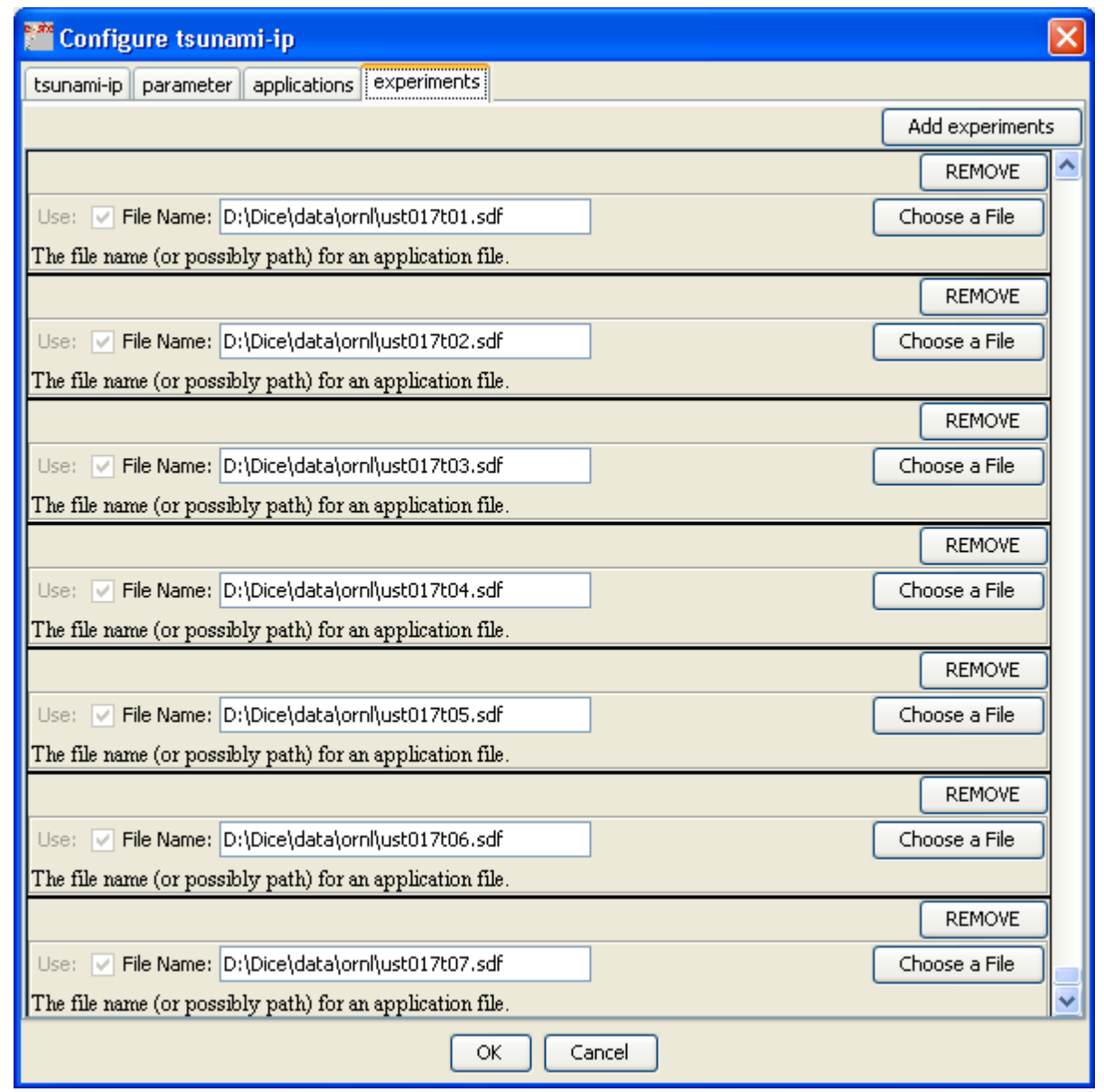

Fig. 103. ExSITE TSUNAMI-IP dialog with handbook experiments loaded.

Items added to the palette can be manipulated or removed by right-clicking in the palette to access a pop-up menu, shown in Fig. 104. 


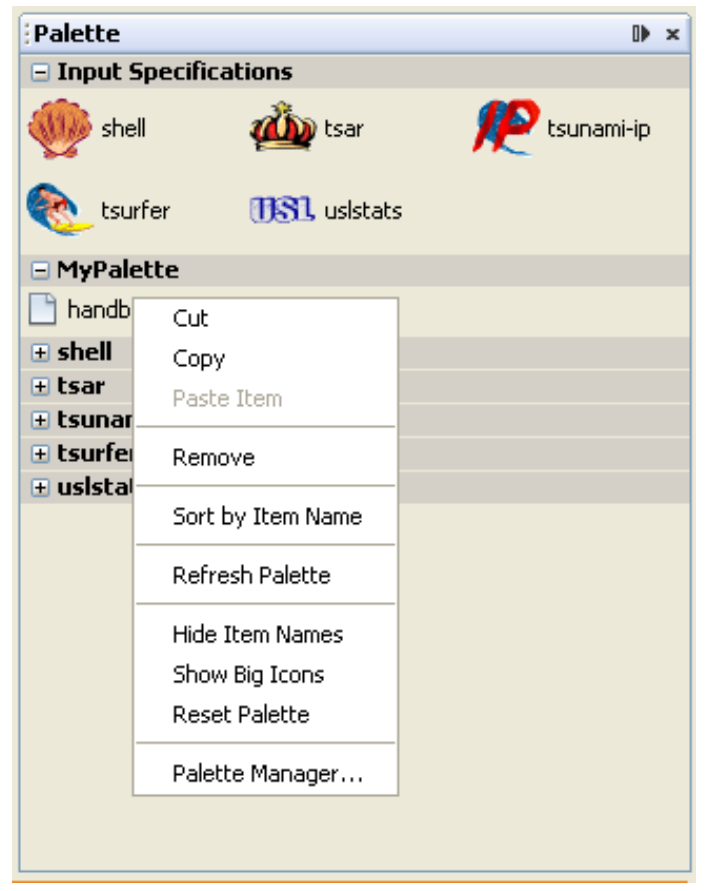

Fig. 104. ExSITE palette with pop-up menu.

A skeleton TSUNAMI-IP input is now prepared to examine the ${ }^{233} \mathrm{U}$ application and assess the similarity of the 281 benchmarks distributed with the ICSBEP Handbook for the validation of this application. To save the input at this point, click the disk icons on the toolbar. Note that ExSITE places an asterisk next to the file name on its tab when unsaved changes have been made to a file.

\subsection{EXAMINATION OF UNCERTAINTIES WITH TSUNAMI-IP}

When beginning a TSUNAMI-IP analysis, it is useful begin by quantifying sources of uncertainty in the application due to uncertainties in the cross-section data. Although the uncertainty information is available from TSUNAMI-1D or -3D calculations, it has not yet been examined for this application. The uncertainty analysis functionality of TSUNAMI-IP will be applied to examine the sources of uncertainty and, therefore, potential sources of computational bias in the ${ }^{233} \mathrm{U}$ application.

\subsubsection{Preparation of TSUNAMI-IP Uncertainty Input with ExSITE}

In ExSITE, double-click on the TSUNAMI-IP icon in the palette to open the TSUNAMI-IP input dialog again. Under the parameter tab, input data are organized by functionality with subtabs. Basic functions, such as the calculation of uncertainties, are available under the Parameter subtab. To request uncertainty edits, select uncert and uncert_long as shown in Fig. 105.

General options regarding cross-section-covariance data are set in the Covariance Data subtab. Click the box next to Cov_fix to request that TSUNAMI-IP correct any potentially missing or corrupted covariance data, and click the box next to use_dcov to substitute default covariance where data are not available on the SCALE cross-section-covariance data file, as shown in Fig. 106. Output edits for TSUNAMI-IP are selected on the Output Edits tab. Here select html to request the 
HTML-formatted output, as shown in Fig. 107. When these settings are completed, click OK to update the TSUNAMI-IP input, and then save the input file. TSUNAMI-IP will update as shown in Fig. 108.

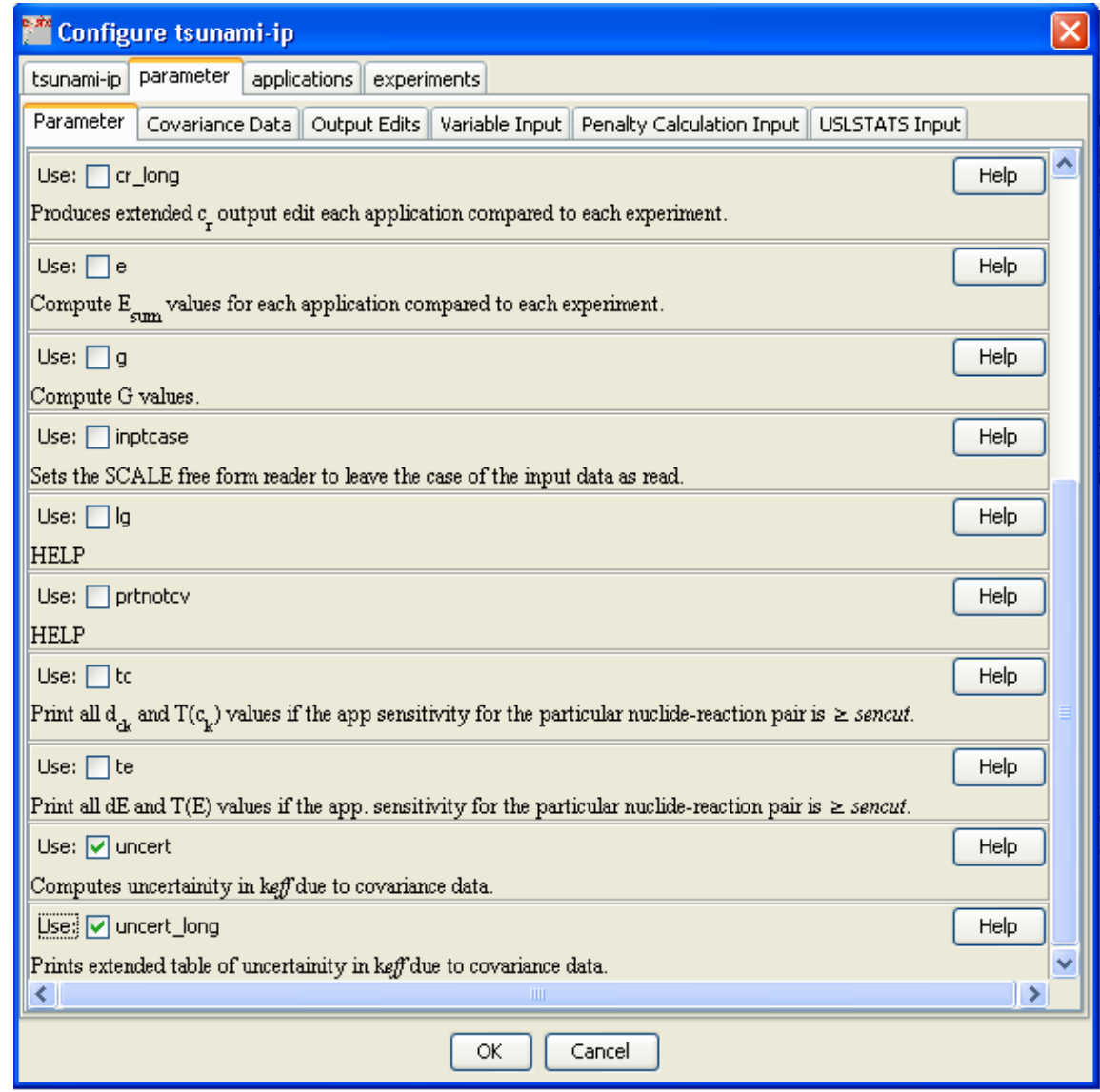

Fig. 105. ExSITE TSUNAMI-IP input dialog for uncertainty data. 


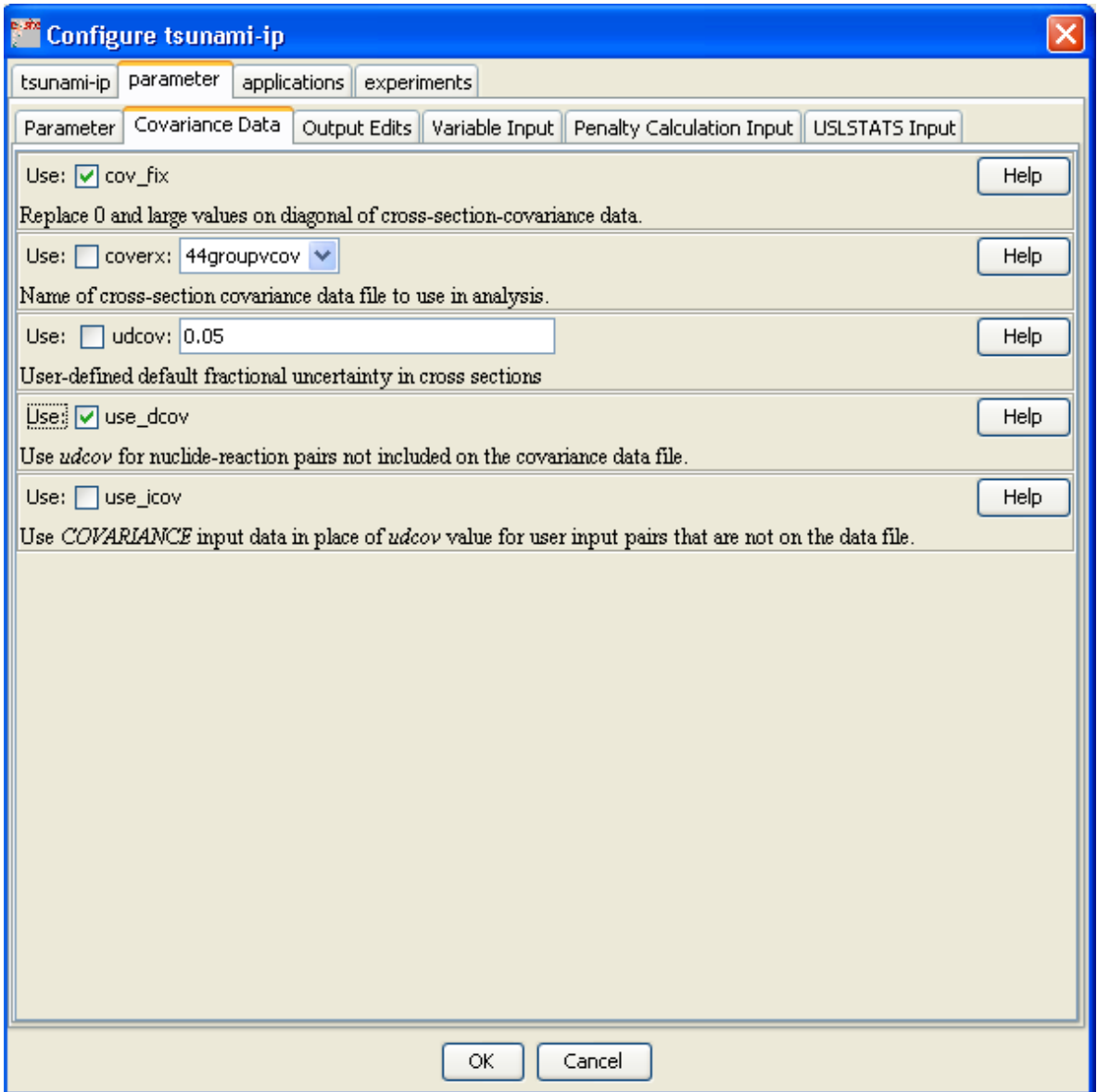

Fig. 106. ExSITE TSUNAMI-IP input dialog for covariance data settings. 


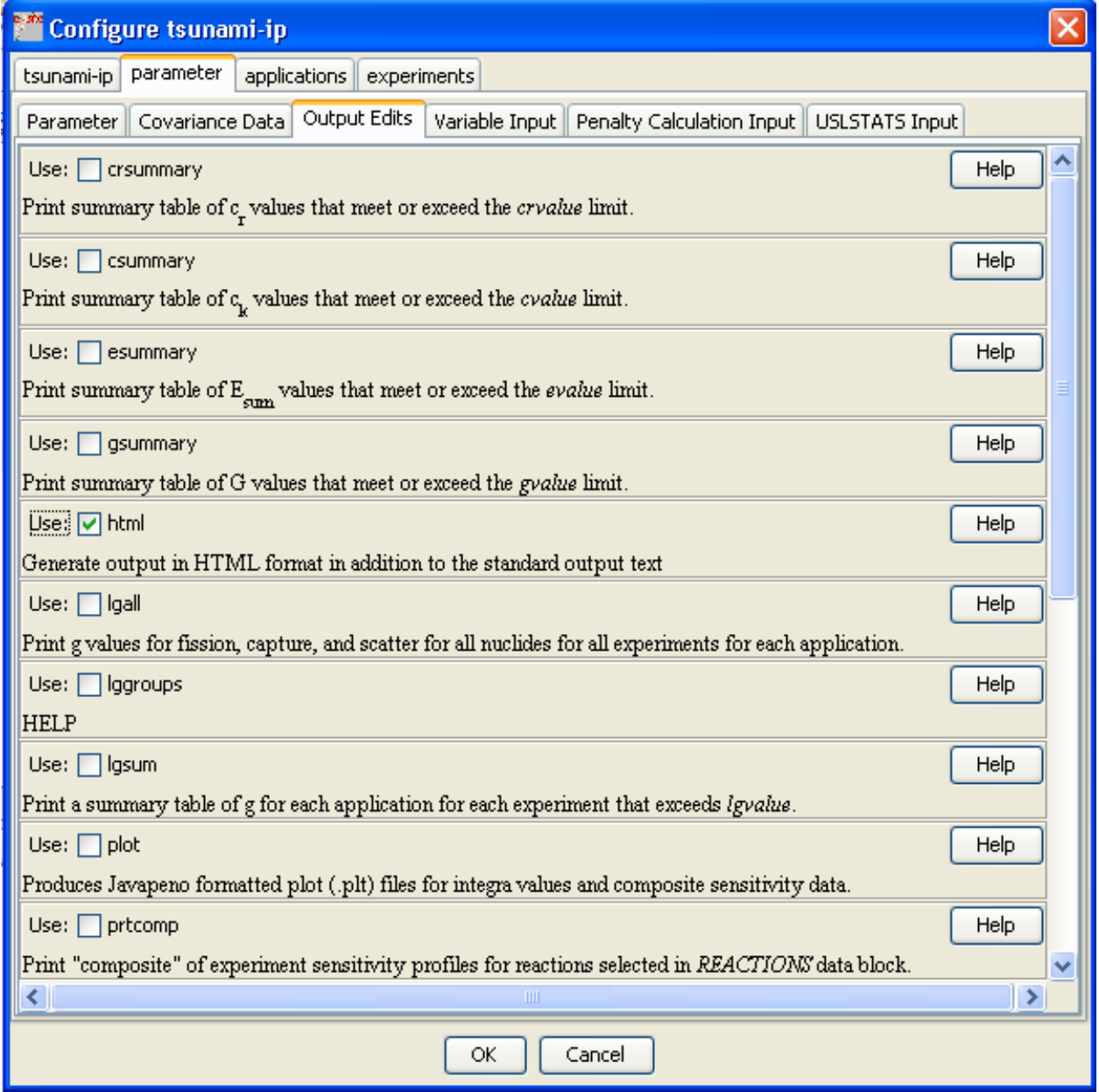

Fig. 107. ExSITE TSUNAMI-IP input dialog for output edit settings. 


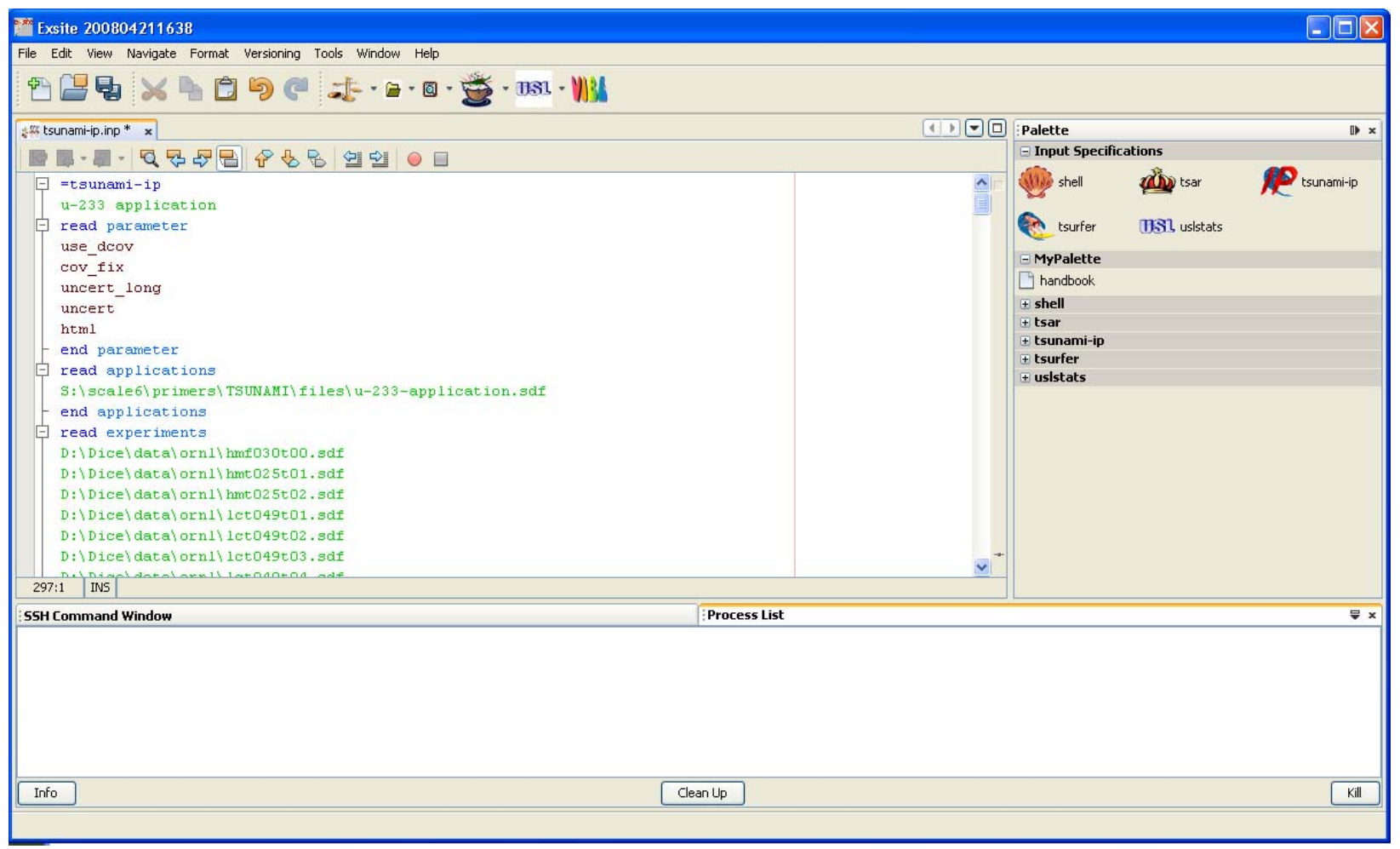

Fig. 108. ExSITE with TSUNAMI-IP input to compute uncertainties.

\subsubsection{Configure ExSITE to Run SCALE 6}

Before executing the TSUNAMI-IP input in SCALE 6, ExSITE must be configured to properly execute it. Clicking on the arrow next to the SCALE icon on the toolbar will reveal a pop-up menu, as shown in Fig. 109. Select Go to Run SCALE Configurations to specify settings for running SCALE. Within ExSITE, multiple run-SCALE configurations can be saved to run different versions of SCALE, run the same version of SCALE with different options, or run SCALE on remote nodes on a network. In this example, ExSITE will be configured to run SCALE 6 under Windows using default settings. In the Configuration dialog, shown in Fig. 110, ensure that the connection type is set to LOCAL, the run command is BATCH6, and the SCALE location is set to the root directory of the SCALE install. Here SCALE 6 is installed on the S: drive, whereas in a typical installation, SCALE is installed on the C: drive. Note that the configuration dialog can also be accessed by selecting Options from the Tools menu.

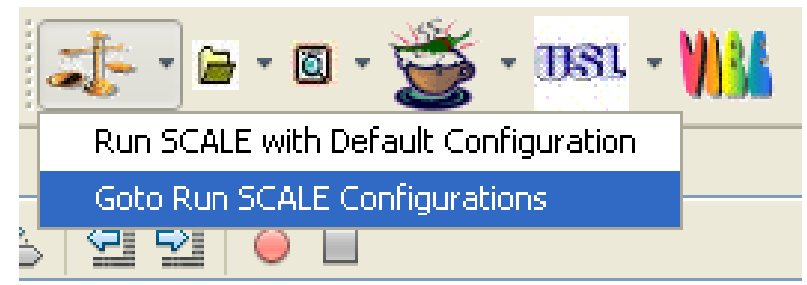

Fig. 109. ExSITE run SCALE menu. 


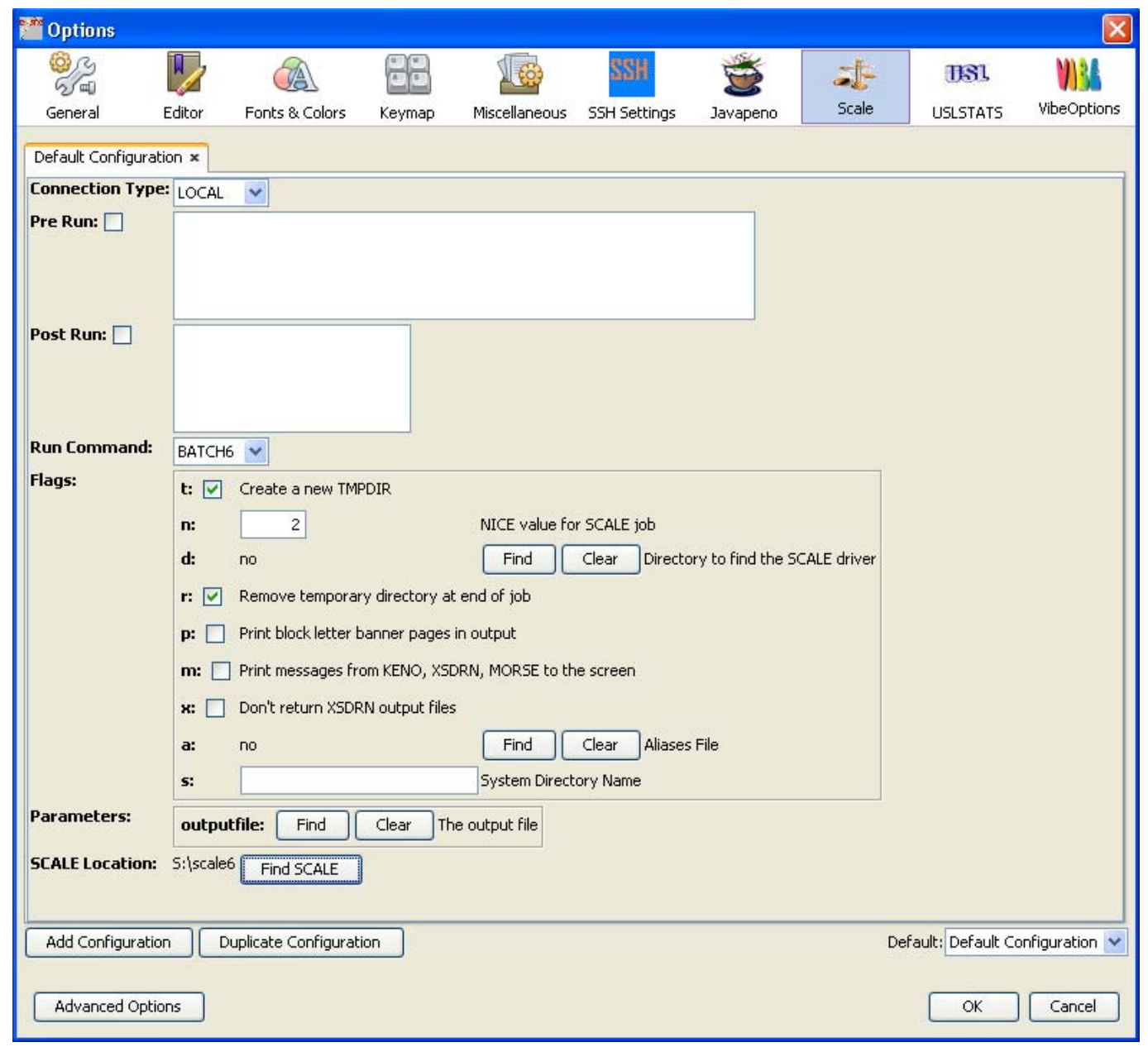

Fig. 110. ExSITE run SCALE configuration dialog.

Continue configuring ExSITE by verifying the settings for Javapeño, USLTATS, and VIBE configurations as shown in Fig. 111, Fig. 112, and Fig. 113, respectively. 


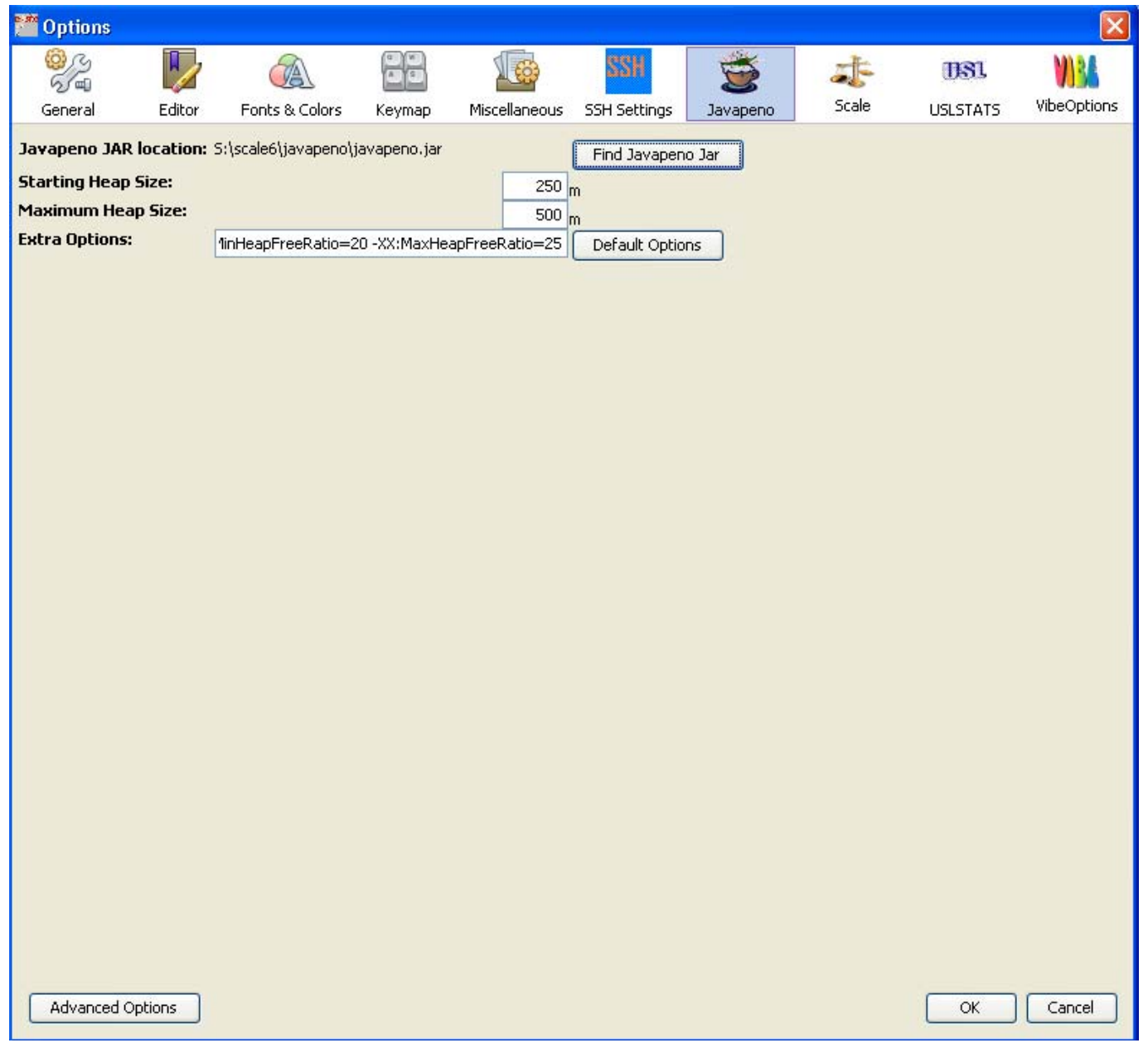

Fig. 111. ExSITE Javapeño configuration dialog. 


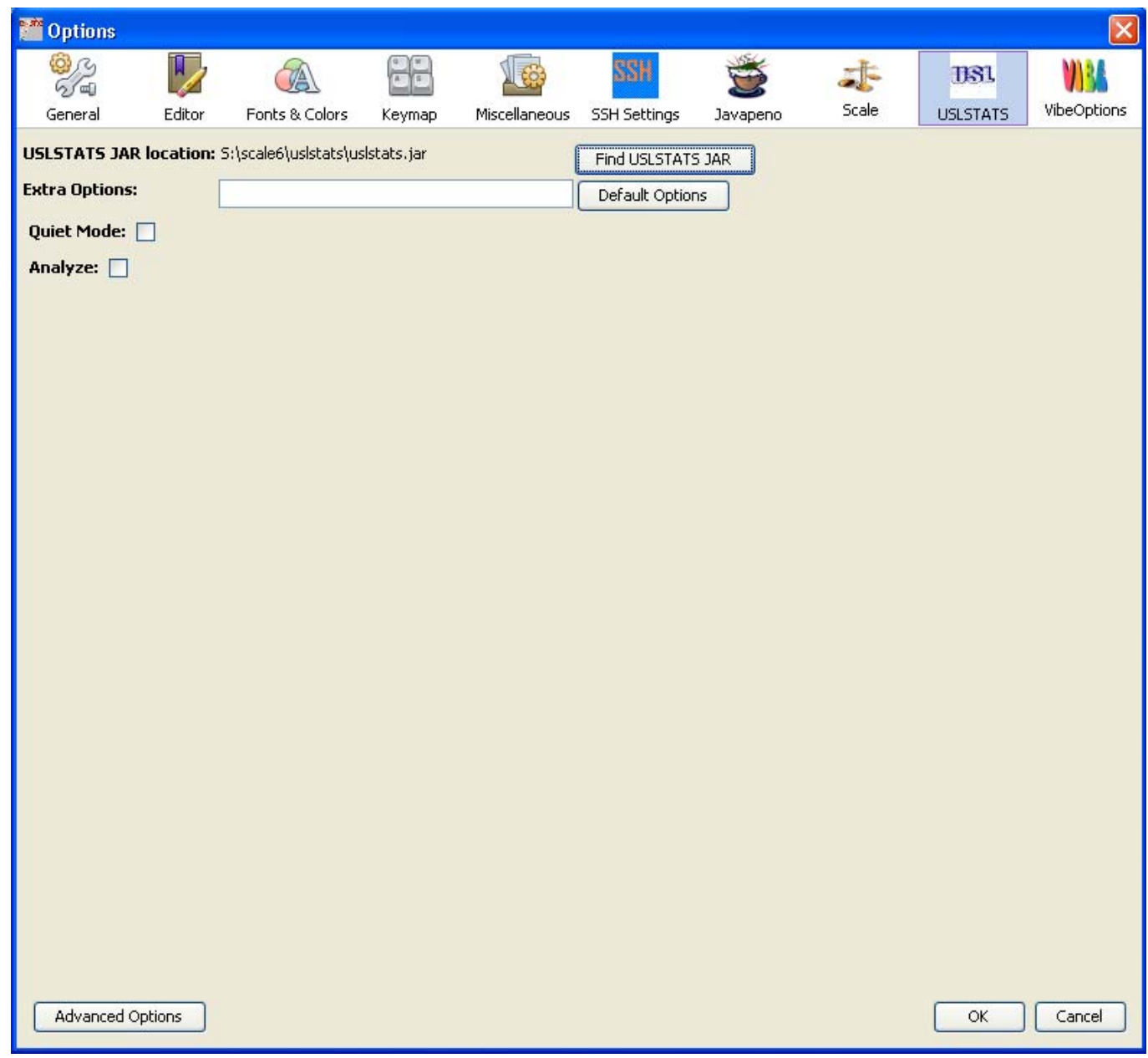

Fig. 112. ExSITE USLSTATS configuration dialog. 


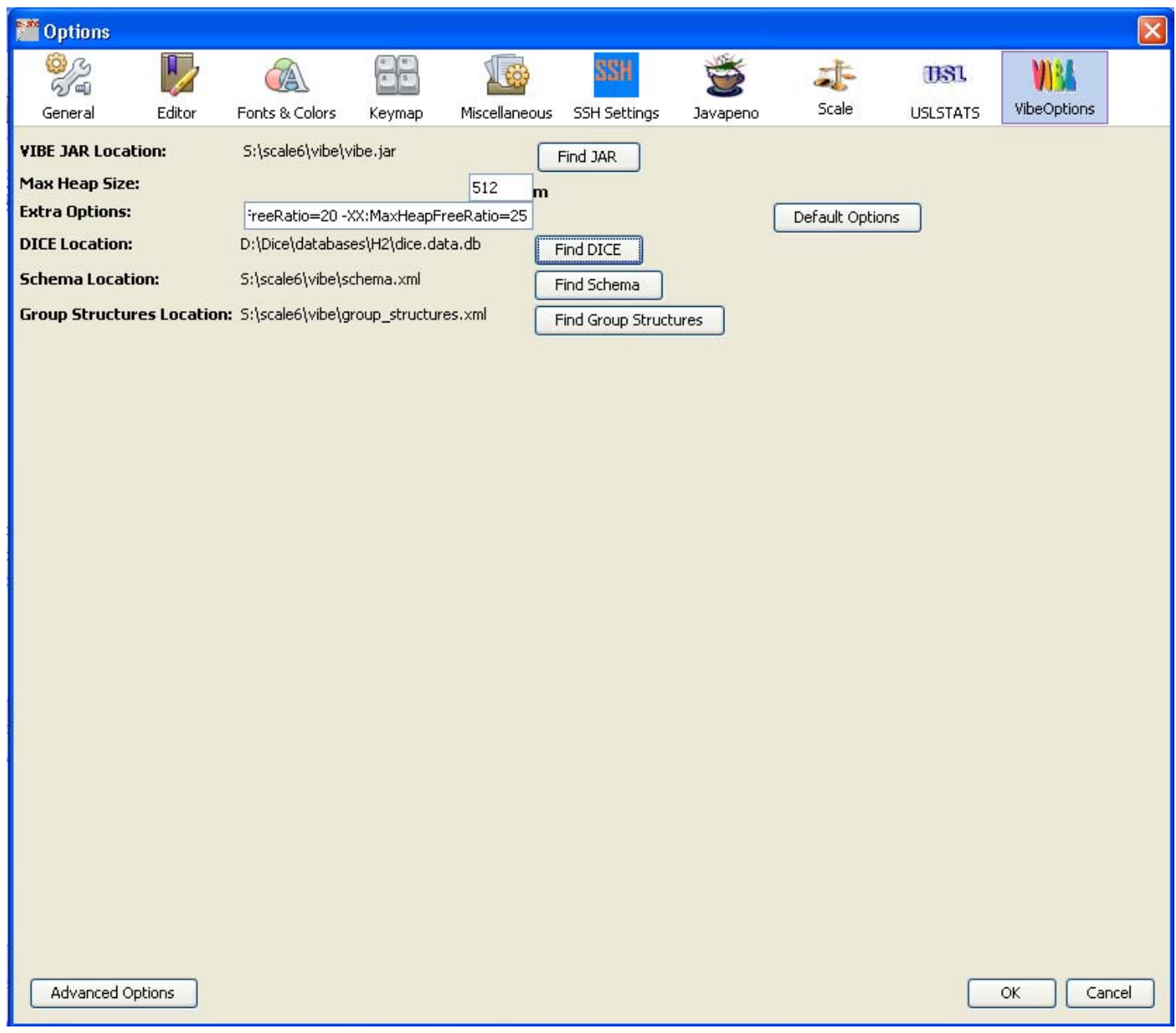

Fig. 113. ExSITE VIBE configuration dialog.

\subsubsection{Using ExSITE to Run TSUNAMI-IP}

To execute SCALE, first select the tab for the input file to run. Here there is only one tab available, the tab for the file tsunami-ip.inp. In general, more than one input file can be open at the same time, and output files or other types of text files can be open simultaneously. To run the tsunami-ip.inp input using the default configuration from the SCALE configurations dialog, simply click the SCALE icon on the toolbar. When the job starts, the Process List tab will update to include the running process, as shown in Fig. 114.

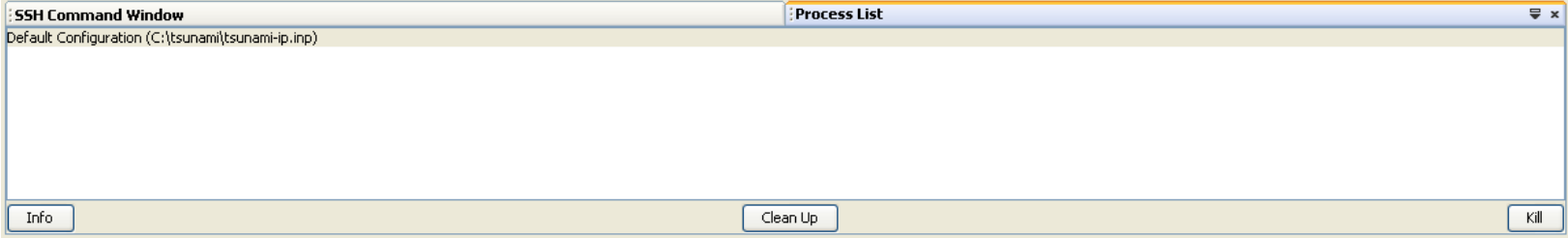

Fig. 114. ExSITE process list window with TSUNAMI-IP input running. 


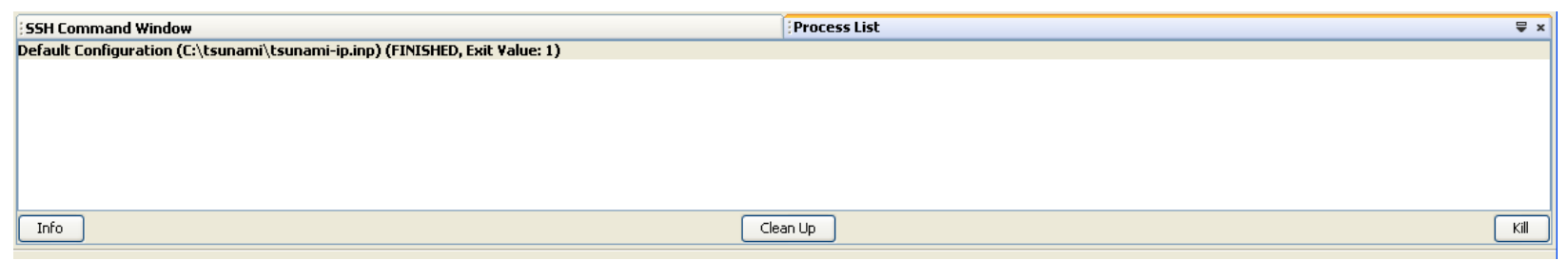

Fig. 115. ExSITE process list window with TSUNAMI-IP input completed.

\subsubsection{Using ExSITE to Open TSUNAMI-IP Output}

The SCALE output files are opened by selecting the desired input file tab in ExSITE, then clicking the output button on the toolbar for the desired output format, as shown in Fig. 116. The plain text output file will open in a new tab in ExSITE, and the HTML output will open in the default web browser. Here click on the HTML output icon to view the TSUNAMI-IP output.

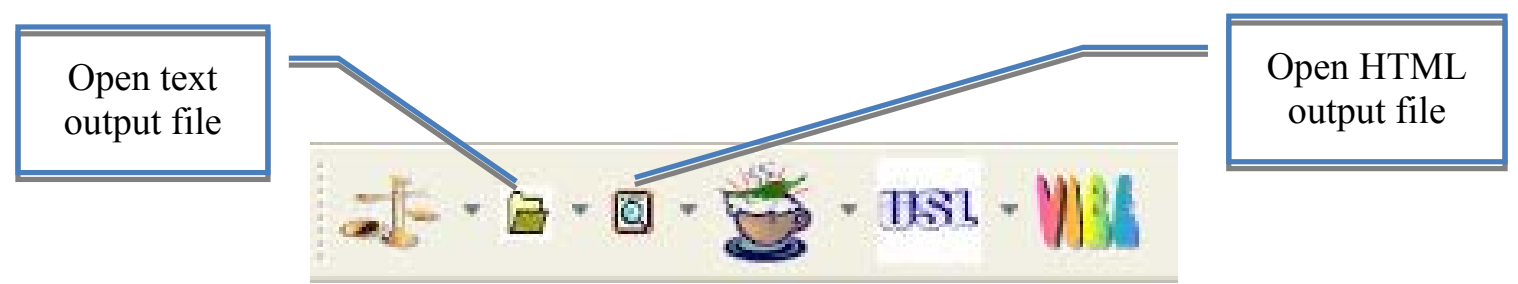

Fig. 116. ExSITE toolbar icons to open output files.

\subsubsection{Viewing TSUNAMI-IP HTML Output}

The HTML output, requested with the TSUNAMI-IP parameter html, will open in the default web browser, which is Internet Explorer in this example, as shown in Fig. 117.

Clicking the the hyperlink for TSUNAMI-IP will open the program verification page for TSUNAMI-IP, where other output edits can be selected from the menu on the left, as shown in Fig. 118.

The warning messages from the code execution can be reviewed by clicking on Messages in the menu on the left side of the screen, then clicking on Warning Messages, as shown in Fig. 119. Here the warning messages report only adjustments made in the cross-section-covariance library because use_dcov and cov_fix were entered in the parameter data.

Under the Input Data submenu in the HTML output, the input parameters are listed, as shown in Fig. 120. Here the input can be verified, and a short description of each input parameter is provided. The Applications List, shown in Fig. 121, provides a brief description of each system identified as an application. Similarly, the Experiments List, shown in Fig. 122, provides a brief description of each system identified as an experimental benchmark. The edit HTML Format Options, shown in Fig. 123, provides a list of options used to format the HTML output. 
File Edit Miew Favorites Tools Help

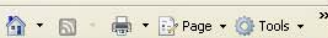

th the escale OUTPUT

SCALE OUTPUT tsunami-ip

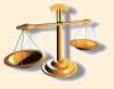

Select an Output Below

u-2.33 application

\section{TSUNAMI-IP}

Comments, Suggestions, User Assistance:

Contact SCALE Help

scalehelp@ornl.gov

or visit the SCALE website

http:/www.ornl.gov/sci/scale

Fig. 117. SCALE initial screen for TSUNAMI-IP HTML output.

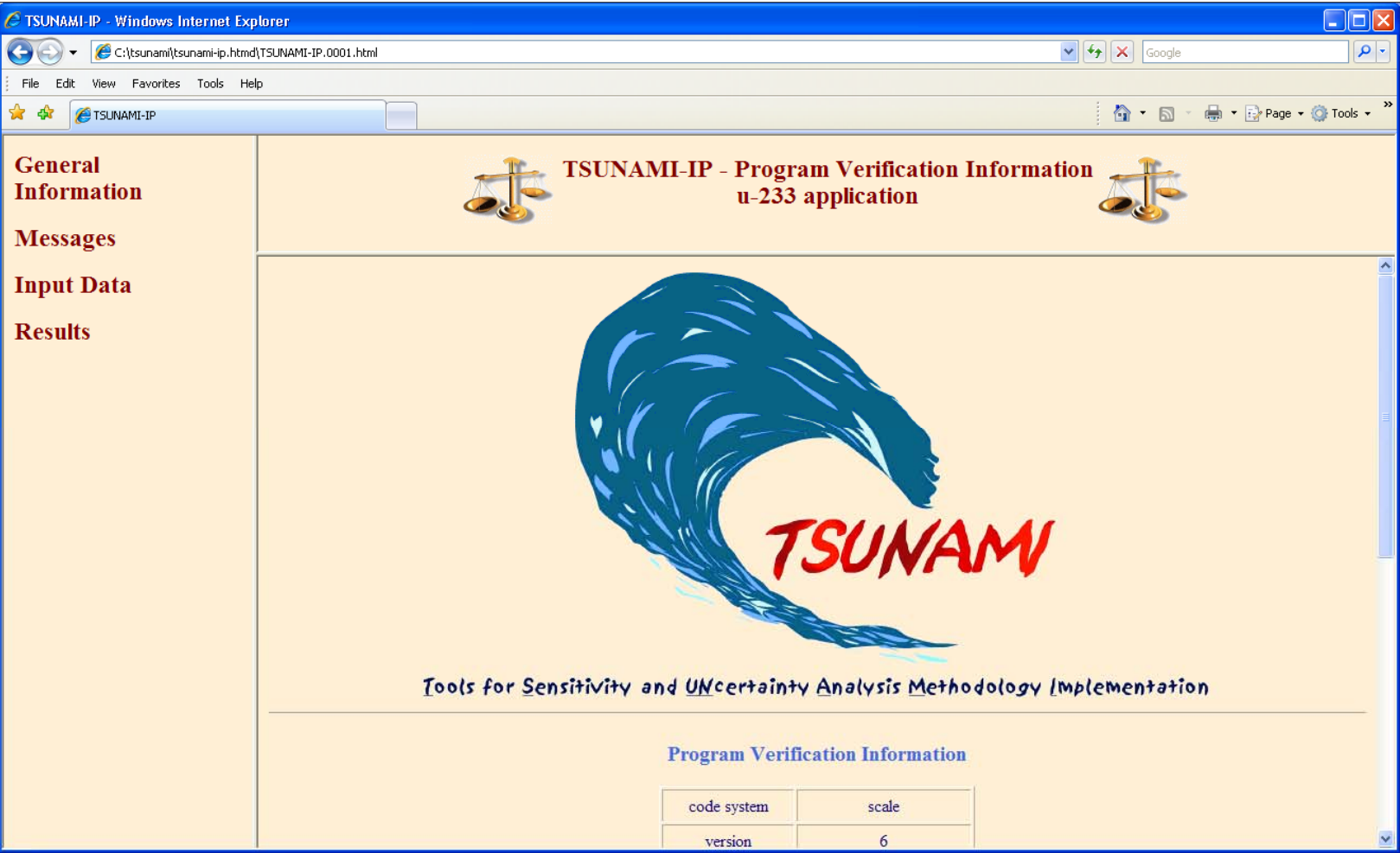

Fig. 118. TSUNAMI-IP program verification page in HTML output. 


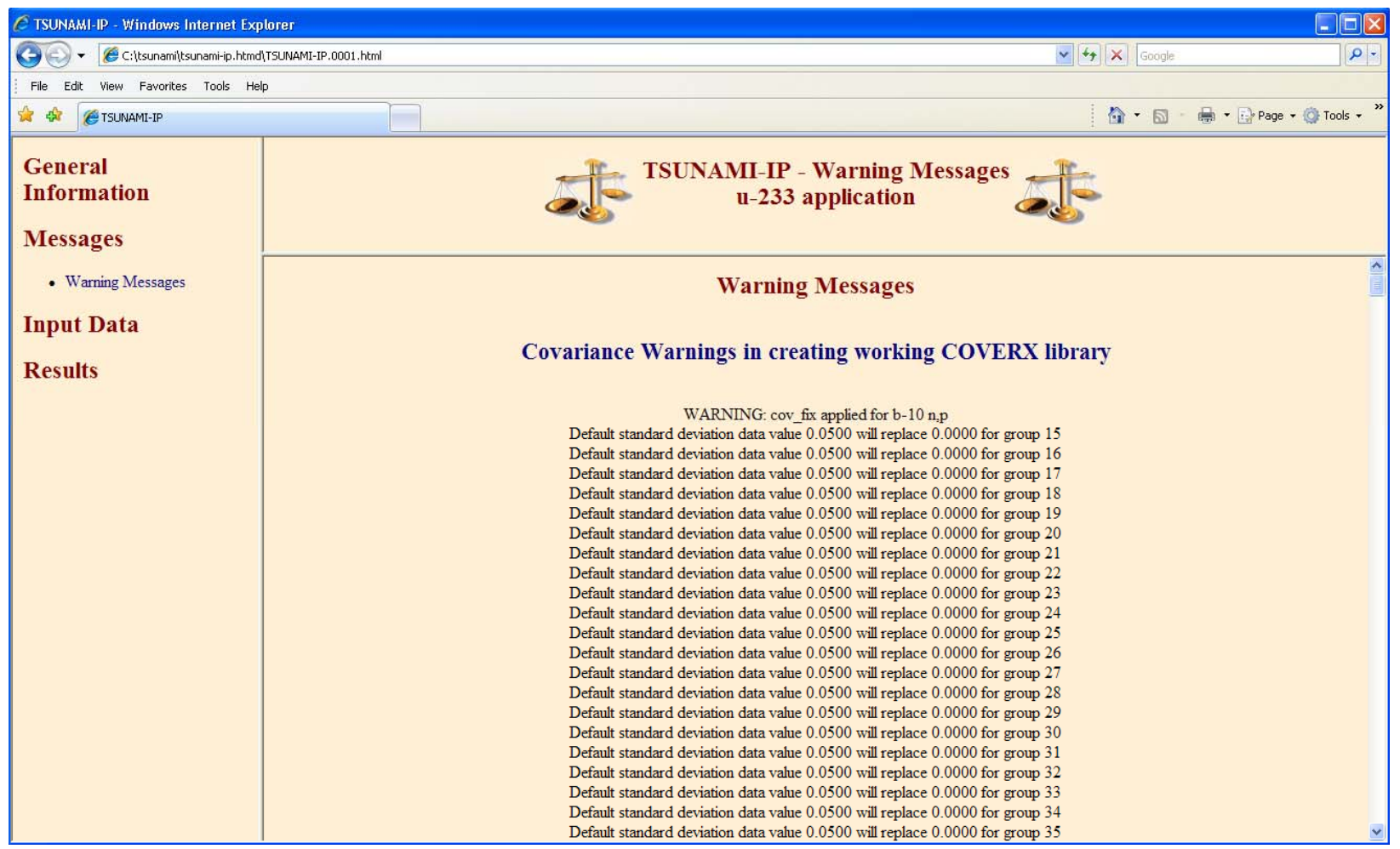

Fig. 119. TSUNAMI-IP warning messages in HTML output.

\begin{tabular}{|c|c|c|c|c|}
\hline \multicolumn{3}{|c|}{ E TSUNAMIIP - Windows Internet Explorer } & \multicolumn{2}{|c|}{$-\square \times$} \\
\hline \multirow{2}{*}{\multicolumn{5}{|c|}{$\begin{array}{l}\text { (9)- Ciltsunamiltsunami-ip.htmdłt TSUNAMI-IP.0001.html } \\
\text { File Edit Wiew Favorites Tools Help }\end{array}$}} \\
\hline & & & & \\
\hline \multicolumn{3}{|c|}{ is the TSUNAMI-IP } & \multicolumn{2}{|l|}{ 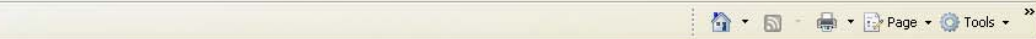 } \\
\hline \multirow{6}{*}{$\begin{array}{l}\text { General } \\
\text { Information } \\
\text { Messages } \\
\quad \text { - Warning Messages } \\
\text { Input Data } \\
\quad \text { - Input Parameters } \\
\text { - Applications List } \\
\text { - Experiments List } \\
\text { - HTML Format Options }\end{array}$} & \multicolumn{4}{|c|}{$\begin{array}{c}\text { TSUNAMI-IP - Input Parameters } \\
\mathrm{u}-233 \text { application }\end{array}$} \\
\hline & \multicolumn{2}{|l|}{ return_work_cor } & 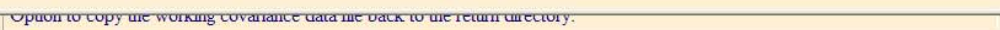 & $\hat{\imath}$ \\
\hline & sencut= & 0.0100 & Cutoff value for ignoring low valued sensitivities in nuclide-reaction specific edit tables. & \\
\hline & senfac $=$ & 0.9000 & $\begin{array}{l}\text { Value used in calculation of completeness parameter. Group-wise sensitivity for the application is counted as validated by the } \\
\text { experiment if the sensitivity from the experiment is greater than the application sensitivity times senfac. }\end{array}$ & \\
\hline & uncert & true & Computes uncertainty in response due to covariance data. & \\
\hline & uncert_long & true & Prints extended table of uncertainty in response due to covariance data sets uncert to true. & \\
\hline \multirow[t]{8}{*}{ Results } & use_dcov & true & $\begin{array}{l}\text { Use user-defined default covariance data, udcov, for nuclide reaction pairs not included on the covariance data file. The user-defined } \\
\text { data will be used in the fourth penalty assessment as well as the computation of } c(k) \text { and uncertainty calculations. }\end{array}$ & \\
\hline & udcov $=$ & 0.0500 & $\begin{array}{l}\text { User-defined default value of standard deviation in cross-section data to use for nuclide-reaction pairs for which covariance data are } \\
\text { not available on the selected data file. }\end{array}$ & \\
\hline & udcov_corr $=$ & 1.0000 & $\begin{array}{l}\text { User-defined default correlation value to use for nuclide-reaction pairs for which covariance data are not available on the selected } \\
\text { data file. }\end{array}$ & \\
\hline & udcov_corr_type $=$ & zone & $\begin{array}{l}\text { User-defined default correlation in cross-section data to use for nuclide-reaction pairs for which covariance data are not available on } \\
\text { the selected data file. (long, zone, short) }\end{array}$ & \\
\hline & udcov_fast $=$ & 0.0000 & $\begin{array}{l}\text { User-defined default value of standard deviation in cross-section data to use for fast data for nuclide-reaction pairs for which } \\
\text { covariance data are not available on the selected data file. }\end{array}$ & \\
\hline & udcov_inter $=$ & 0.0000 & $\begin{array}{l}\text { User-defined default value of standard deviation in cross-section data to use for intermediate data for nuclide-reaction pairs for which } \\
\text { covariance data are not available on the selected data file. }\end{array}$ & \\
\hline & udcov_therm $=$ & 0.0000 & $\begin{array}{l}\text { User-defined default value of standard deviation in cross-section data to use for thermal data for nuclide-reaction pairs for which } \\
\text { covariance data are not available on the selected data file. }\end{array}$ & \\
\hline & use_diff_groups & false & Allow sensitivity data files to have different energy group structures. & v \\
\hline
\end{tabular}

Fig. 120. TSUNAMI-IP input parameters in HTML output. 


\begin{tabular}{|c|c|c|c|c|c|}
\hline \multicolumn{4}{|c|}{ E TSUNAMIIP - Windows Internet Explorer } & 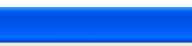 & D回品 \\
\hline \multirow{2}{*}{\multicolumn{6}{|c|}{ 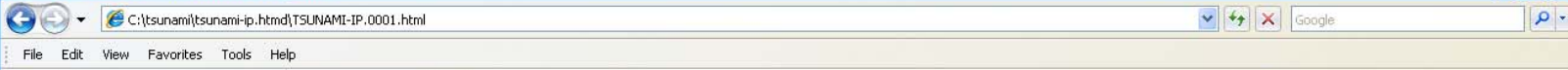 }} \\
\hline & & & & & \\
\hline \multicolumn{6}{|c|}{ to को TSUNAMIIP } \\
\hline \multirow{6}{*}{$\begin{array}{l}\text { General } \\
\text { Information } \\
\text { Messages } \\
\quad \text { - Warning Messages } \\
\text { Input Data } \\
\quad \text { Input Parameters } \\
\text { Applications List } \\
\text { - Experiments List } \\
\text { HTML Format Options } \\
\text { Results }\end{array}$} & \multicolumn{5}{|c|}{$\underset{\text { u-233 application }}{\pi}$ TSUNAMI-IP - User Input Applications in this Analysis } \\
\hline & \multicolumn{5}{|c|}{ User Input Applications Used in this Analysis } \\
\hline & APPLICATION & DESCRIPTION & & & \\
\hline & \multirow{3}{*}{1} & \multicolumn{3}{|c|}{ Tittle: app. 1 - 220 gu2331 no excess acid } & \\
\hline & & Name: app. 1 - 220 gu233/ & Type: keff & Format: Relative & \\
\hline & & Filename: S: Iscale6 primers ISUNAMI files u-233-application.sdf & 238 groups & 199 Sensitivity profiles & \\
\hline
\end{tabular}

Fig. 121. TSUNAMI-IP applications list in HTML output.

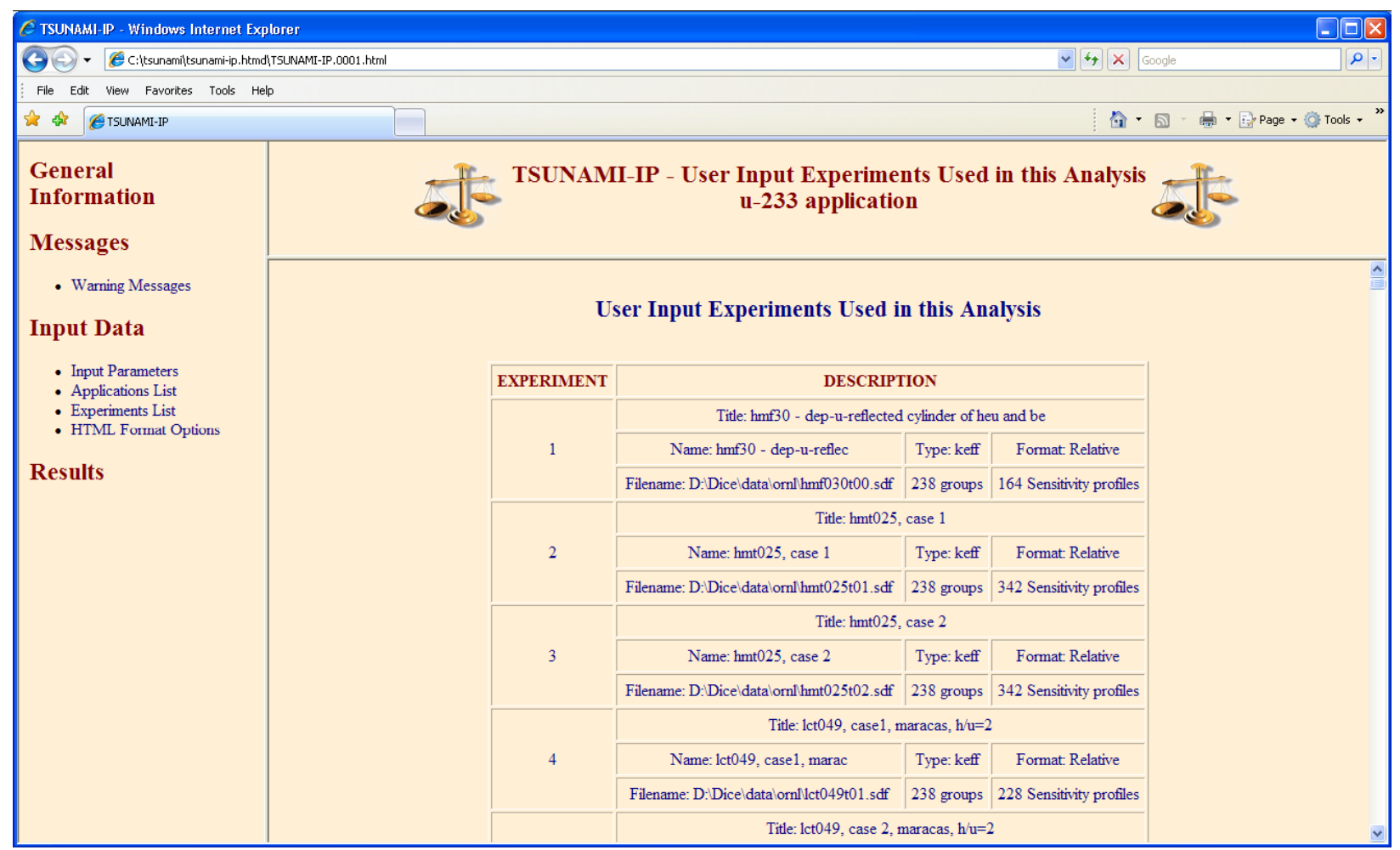

Fig. 122. TSUNAMI-IP experiments list in HTML output. 


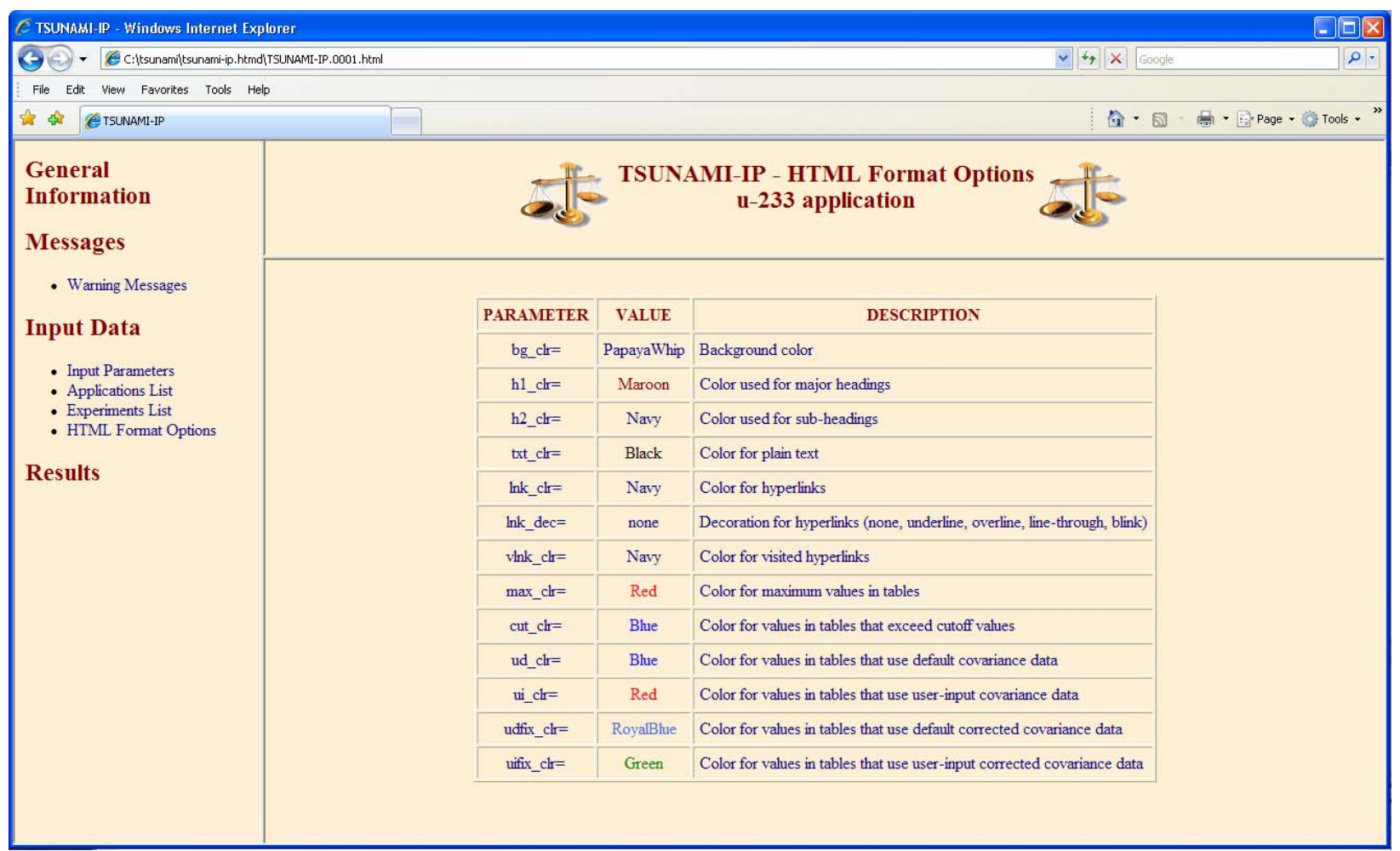

Fig. 123. TSUNAMI-IP HTML format options in HTML output.

The Results section of the HTML output shows the results requested by the TSUNAMI-IP parameter data. In this initial calculation, only the Extended Uncertainty Assessment is listed under the Penalty and Uncertainty Assessment submenu, as shown in Fig. 124. Here the uncertainty in $k_{\text {eff }}$ of the application is $1.067 \% \Delta \mathrm{k} / \mathrm{k}$. This indicates that to a $1 \sigma$ confidence, the relative bias of the application is expected to be less than $1.067 \%$. In this extended edit, the contributions to this uncertainty from each covariance matrix are listed in order of descending magnitude. Here the primary contributors to uncertainty are ${ }^{233} \mathrm{U}$ chi, ${ }^{1} \mathrm{H}$ elastic, and ${ }^{16} \mathrm{O}$ elastic. Uranium-233 nubar, identified with the highest sensitivity, is the fifth highest source of uncertainty in $k_{\text {eff }}$ due to uncertainties in the cross sections. However, ${ }^{233} \mathrm{U}$ contributes four of the top six sources of uncertainty. Further understanding of the sources of uncertainty can be gained by plotting the sensitivity data for these top contributors and the covariance data for the same contributors using Javapeño. 


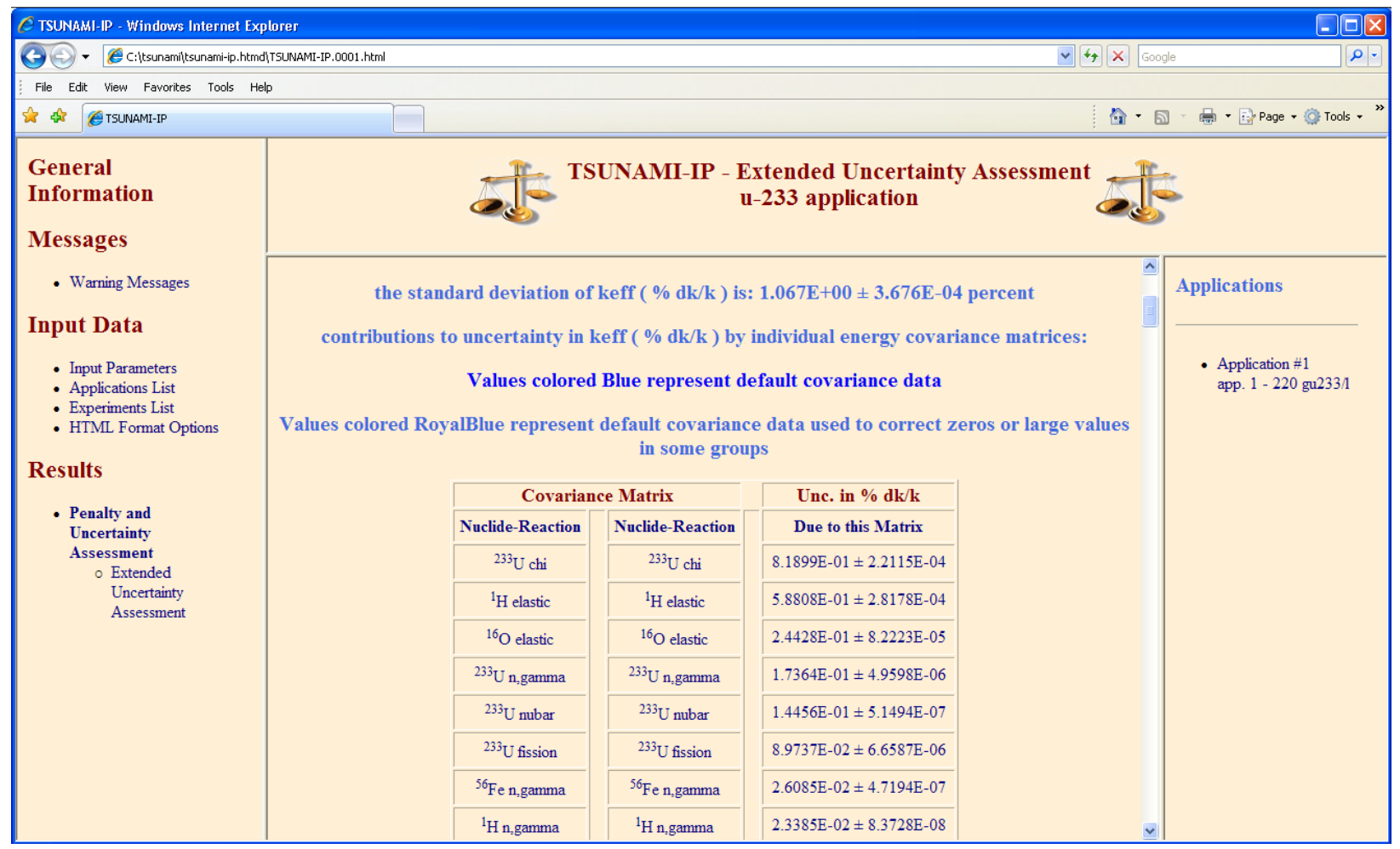

Fig. 124. TSUNAMI-IP extended uncertainty edit in HTML output.

\subsubsection{Viewing Sensitivities and Uncertainties with Javapeño}

Returning to Javapeño, which will still have the data files open from Sect. 8.3, prepare a plot of the top six contributors to uncertainty for the ${ }^{233} \mathrm{U}$ application, as shown in Fig. 125. To view the uncertainties in the cross section, use Javapeño to open the 44groupcov covariance file, which is available in the S: \scale6 $\backslash$ data directory. Select Open Dataset... from the Javapeño File menu; then browse to the 44groupcov file, as shown in Fig. 126; select the file; and click Open. To plot the standard deviation of ${ }^{233} \mathrm{U}$ chi as a function of energy, right-click on the covariance matrix $u-233$-chi to u-233-chi and select Plot Std. Dev. By Energy in New Window, as shown in Fig. 127. To add the other standard deviations to same window, right-click the desired matrix and select Plot Std. Dev. By Energy, as shown in Fig. 128. The standard deviations for the six top contributors to uncertainty in the ${ }^{233} \mathrm{U}$ application are shown in Fig. 129.

Examining Figs. 125 and 129 together, it can be observed that ${ }^{233} \mathrm{U}$ chi and ${ }^{233} \mathrm{U}$ nubar have the highest sensitivities, followed by the high-energy sensitivity for ${ }^{1} \mathrm{H}$ elastic scattering. However, ${ }^{233} \mathrm{U} \mathrm{n}$, gamma has the highest uncertainty in the fast region. In a prototype version of Javapeño, to be released through the SCALE website after the release of SCALE 6, the sensitivities shown in Fig. 125 can be multiplied by the uncertainties shown in Fig. 129 to form an energy-dependent approximation to the uncertainty in $k_{\text {eff }}$ due to uncertainties in the cross sections, as shown in Fig. 130. The data shown in this plot are approximate because only the standard deviation in the cross section is considered. The off-diagonal terms of the covariance matrices are neglected. However, these types of approximate plots provide insight into the energy dependence of the uncertainty. In Fig. 130, the uncertainty of $k_{\text {eff }}$ due to ${ }^{233} \mathrm{U}$ chi, using the unconstrained chi calculation, is observed at high energies. There is also a significant contribution from ${ }^{1} \mathrm{H}$ and ${ }^{16} \mathrm{O}$ elastic at high energies. Although this is a thermal application, the 
${ }^{233} \mathrm{U}$ n,gamma, nubar, and fission contributions to $k_{\text {eff }}$ uncertainty arise from cross-section uncertainties at both thermal and intermediate energies.

Exercises of this type are useful in understanding potential sources of bias caused by errors in the cross sections. Validation of the application with similar critical experiments, where available, will quantify the value of the bias within the bias uncertainty.

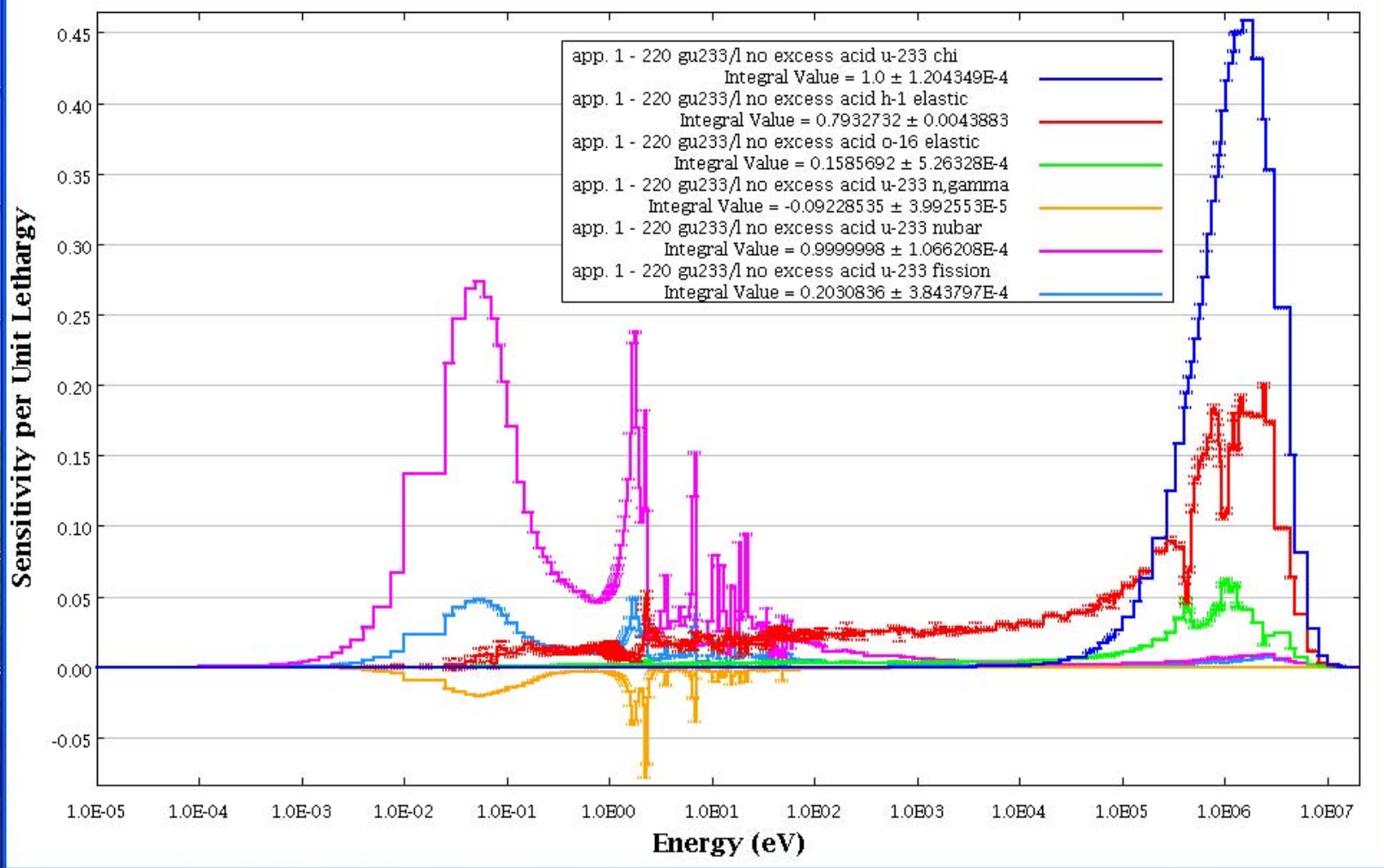

Fig. 125. Javapeño plot of sensitivities from ${ }^{233} \mathrm{U}$ application for top six contributors to uncertainty. 


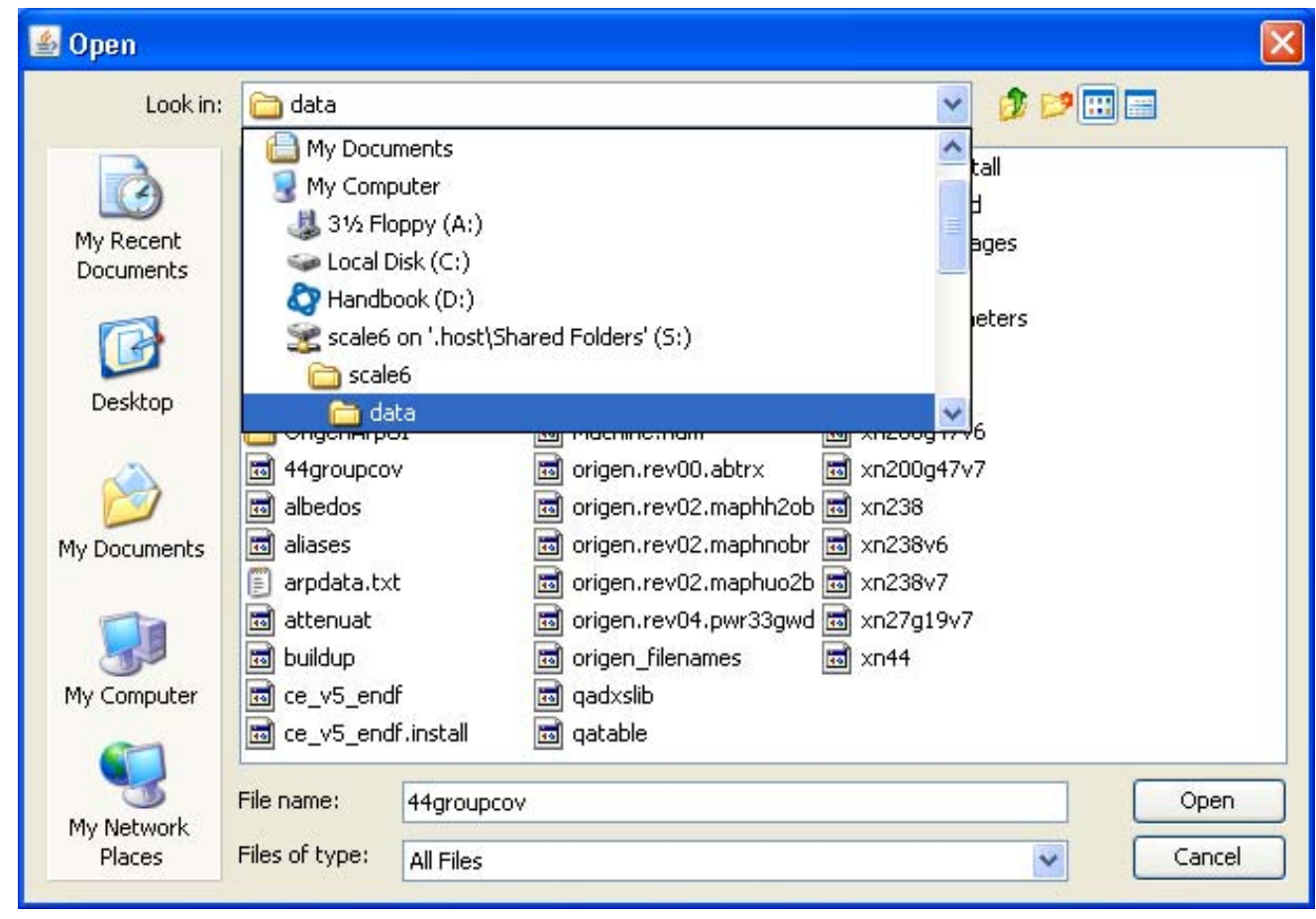

Fig. 126. Javepeño file chooser to open "44groupcov" covariance file.

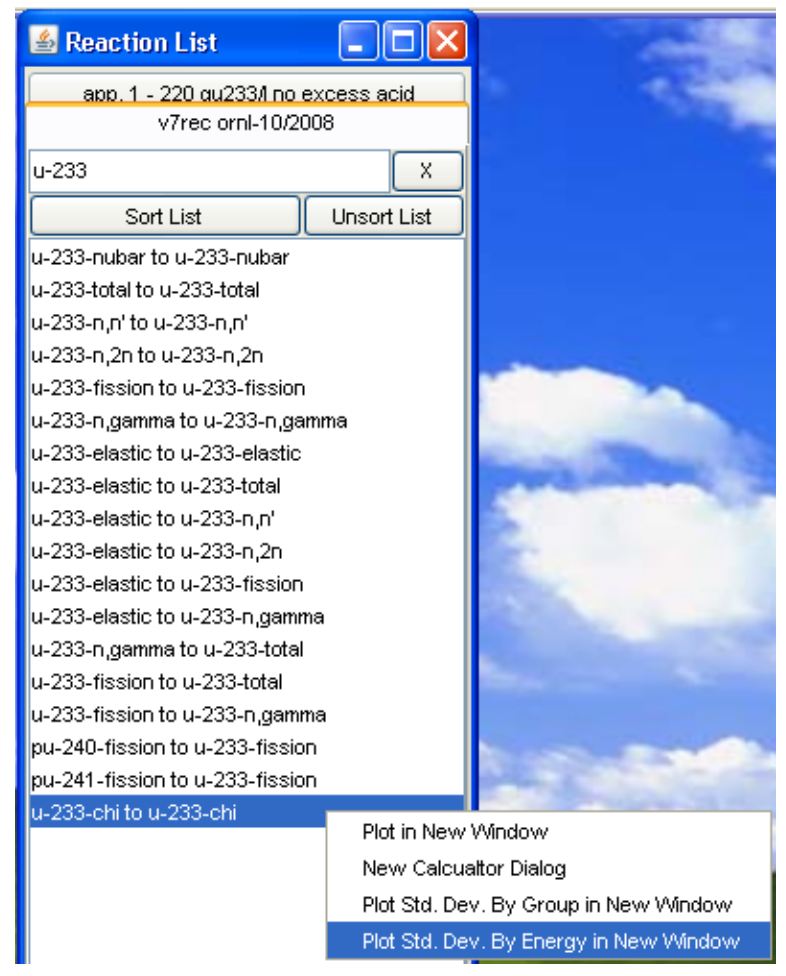

Fig. 127. Plotting standard deviation of cross-section-covariance matrix in a new window using Javapeño. 


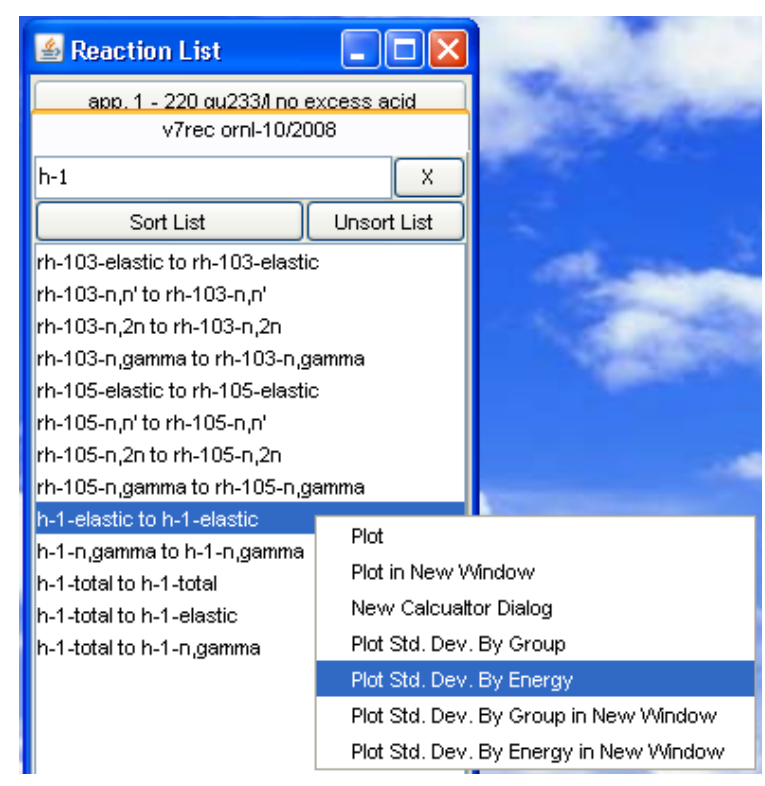

Fig. 128. Plotting standard deviation of cross-section-covariance matrix using Javapeño.

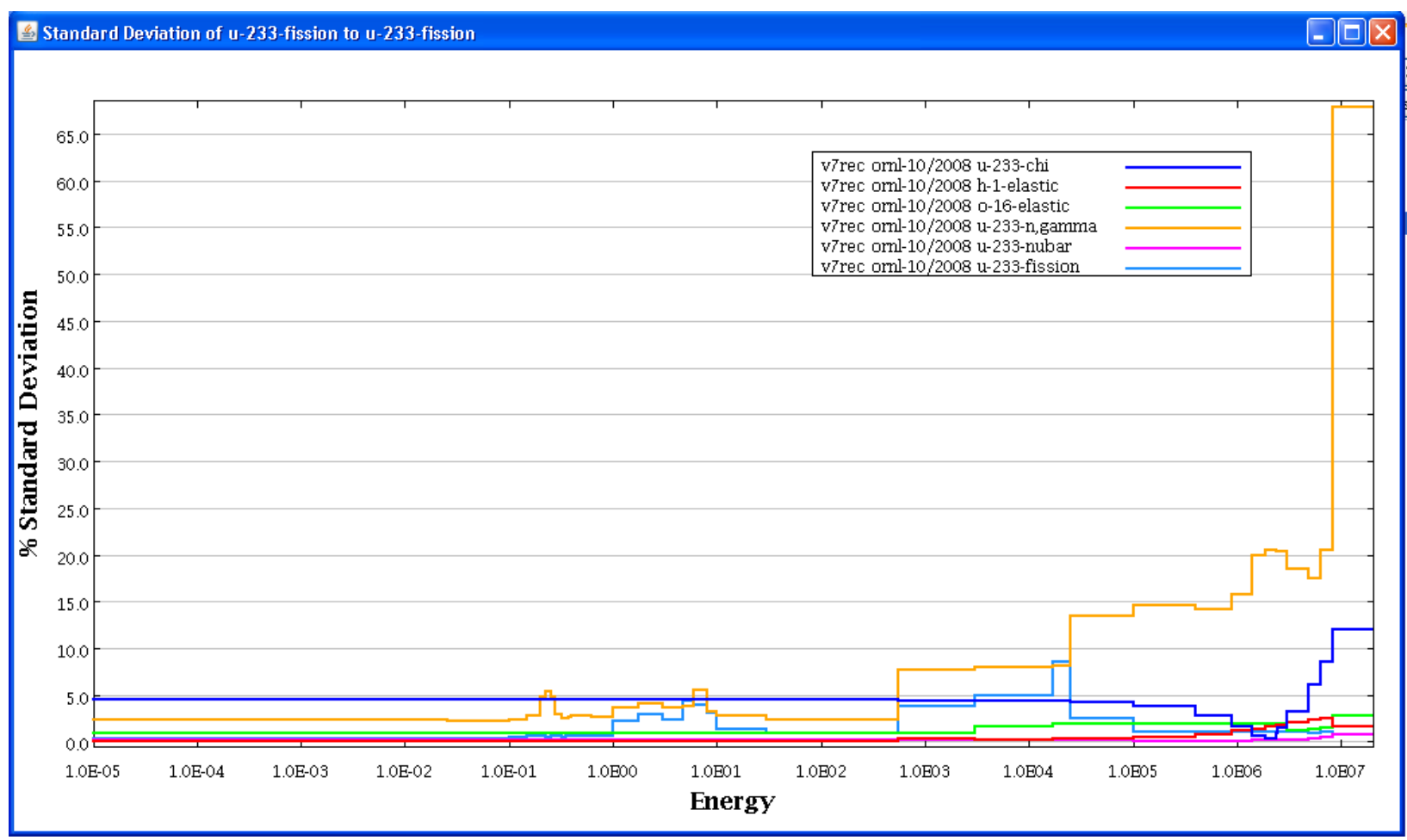

Fig. 129. Javapeño plot of standard deviations of cross sections for ${ }^{233} \mathrm{U}$ application. 


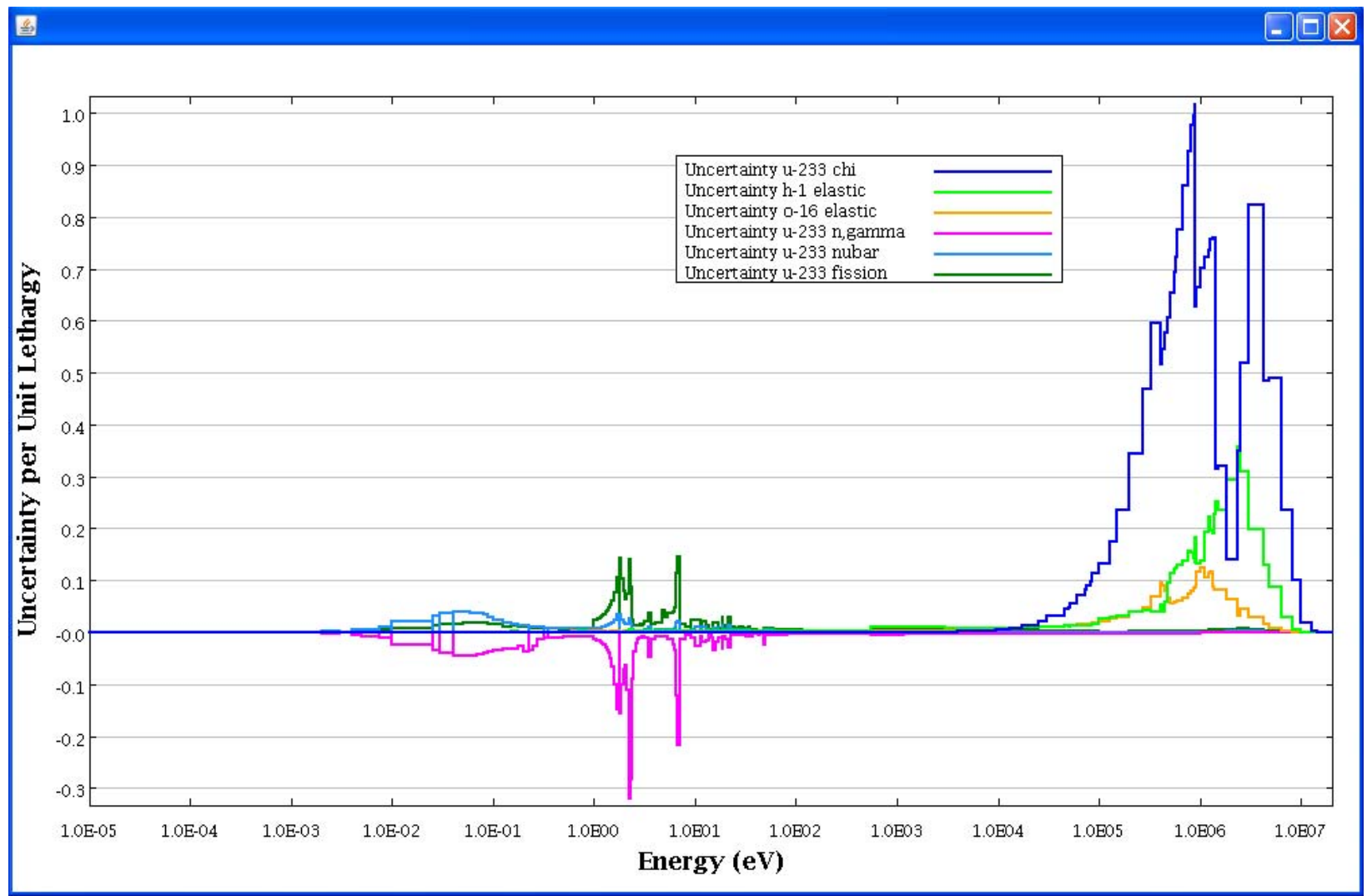

Fig. 130. Energy-dependent uncertainty plot created with post-SCALE 6 Javapeño.

\subsection{EXAMINATION OF GLOBAL SIMILARITY WITH $c_{k}$}

A widely used index for similarity assessment is the correlation of $k_{\text {eff }}$ uncertainties, known as $c_{k}$. The $c_{k}$ index quantifies the amount of shared uncertainty in the $k_{\text {eff }}$ values of an application and a benchmark due to cross-section uncertainties. A $c_{k}$ value of 1.0 means that the uncertainties for the application and the benchmark are all generated from the same nuclides and reactions at the same energies, whereas a $c_{k}$ value of 0.0 means that uncertainties of the two systems are completely unrelated. To request the calculation of $c_{k}$ values from TSUNAMI-IP, simply add the letter $c$ to the TSUNAMI-IP input. With $c$ added to the input, TSUNAMI-IP will calculate the $c_{k}$ values so they will be available for use in output edits or as screening criteria for other assessments. However, the $c_{k}$ values will be printed only if an output edit is requested. If all $c_{k}$ values are desired, enter values in the parameter data. If only a summary of values that exceed a threshold criteria is desired, enter csummary in the parameter data. In the summary edit, only experiments with $c_{k}$ values that meet or exceed the value set with the cvalue= parameter are listed. By default, the threshold criterion is set to 0.9 , so only experiments with a similarity to the application of at least $90 \%$ will be listed.

To request TSUNAMI-IP to calculate and print the $c_{k}$ values through ExSITE, either type $c$, csummary, and values, in parameter block of the input, or toggle these parameter inputs using the palette. A convenient way to directly access the parameter palette is to open the subpalette for TSUNAMI-IP by clicking on the + next to tsunami-ip near the bottom of the palette, then drag the TSUNAMI-IP palette icon parameter into the parameter section of the input file, as shown in Fig. 131. A palette dialog will open that shows only TSUNAMI-IP parameter data. In the Parameter subtab, check the 
box next to c, as shown in Fig. 132. In the Output Edits subtab, check the boxes next to csummary and values, then click OK. ExSITE will update the TSUNAMI-IP input as shown in Fig. 133.

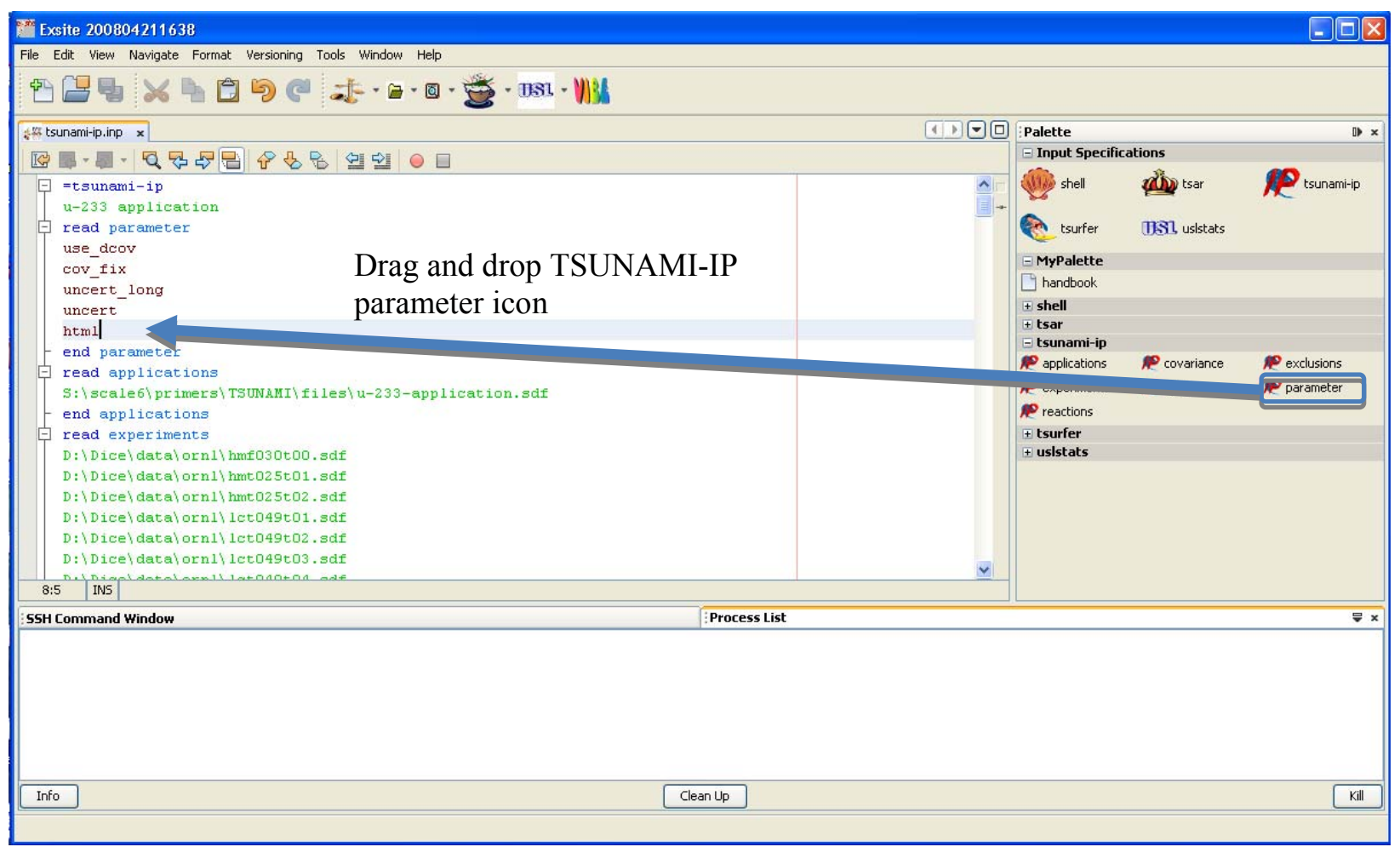

Fig. 131. Opening TSUNAMI-IP parameter palette in ExSITE. 


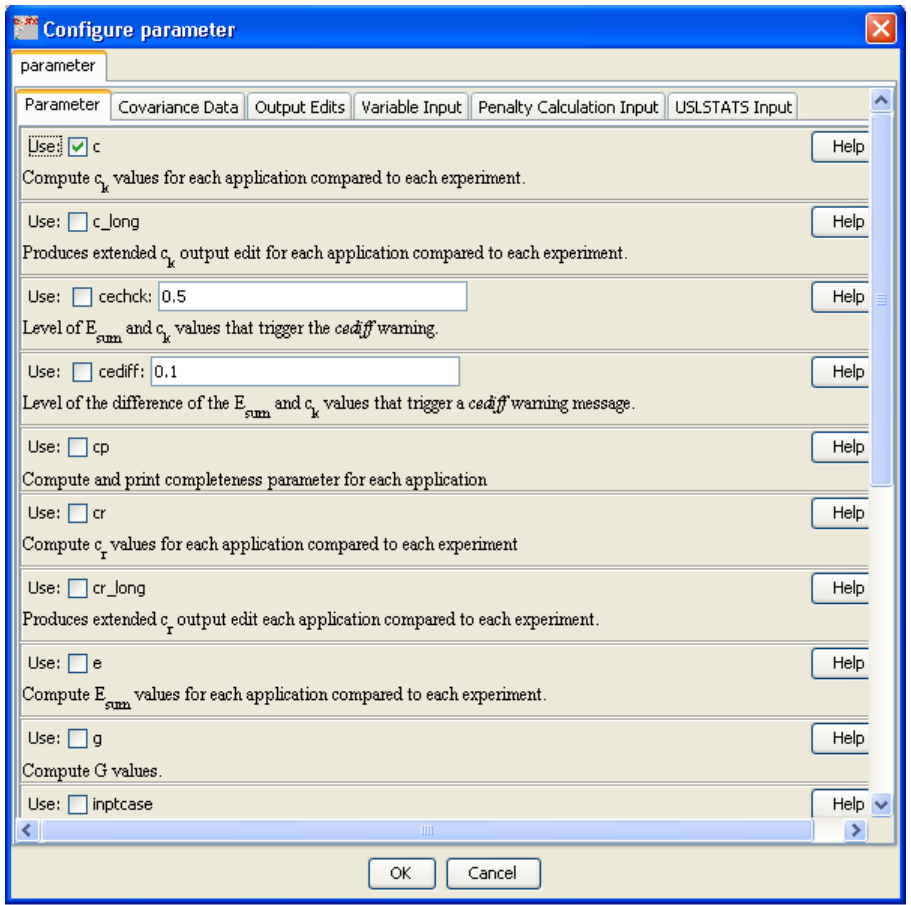

Fig. 132. ExSITE palette dialog for TSUNAMI-IP parameter input.

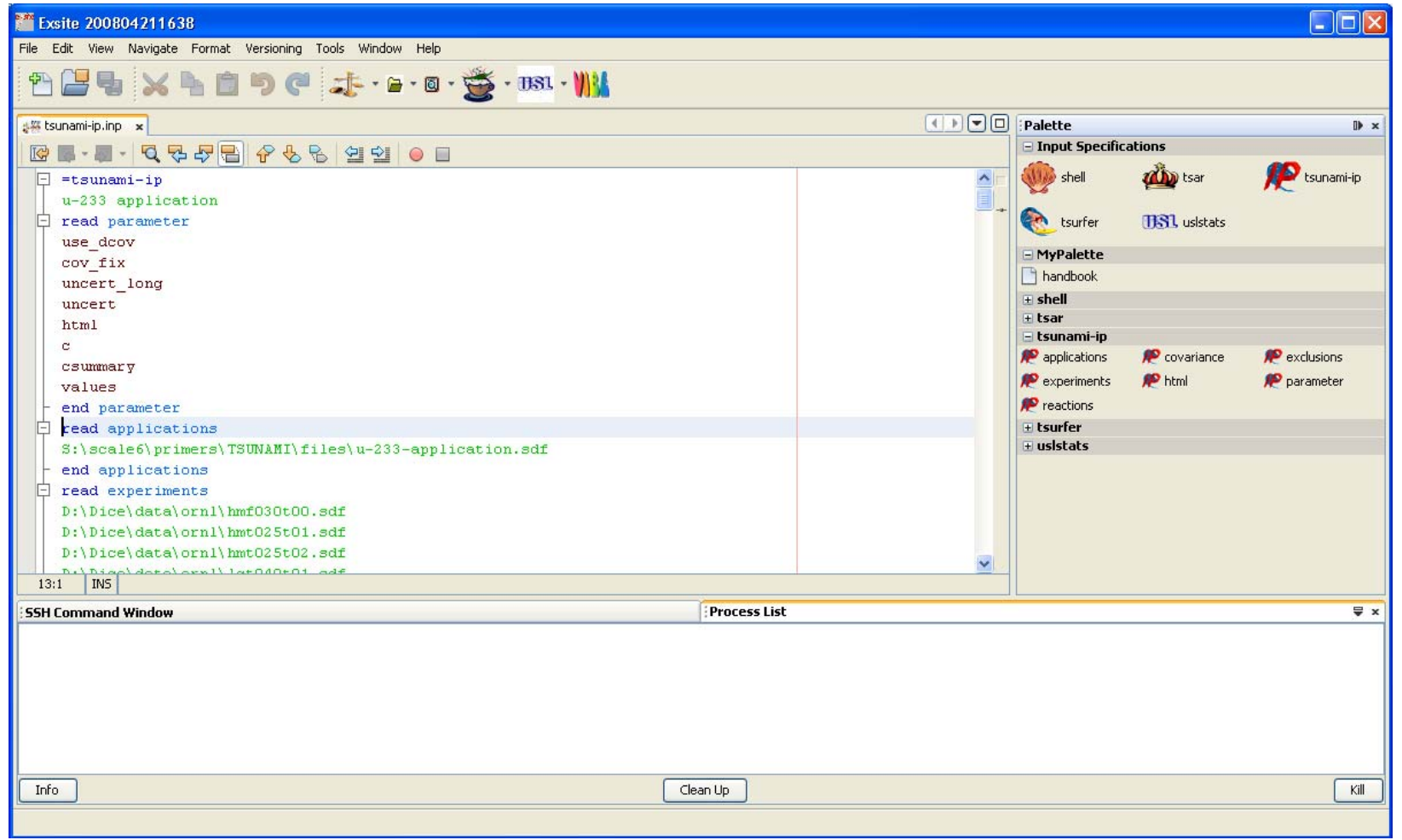

Fig. 133. ExSITE with TSUNAMI-IP input updated to compute and print $c_{k}$ values. 
To rerun the input with the updated parameters, click the SCALE icon. When the process list indicates the job is complete, refresh the HTML output to view the additional output edits. As shown in Fig. 134, the Results section now contains a new submenu for Global Integral Indices. Here the Integral Index Values edit shows the results for each experiment compared to the application. This is the output edit requested with the values parameter. If more than one application had been input, the menu on the right side of the browser would provide access to the output edit for each application. The columns of the values output table can be sorted by clicking on the column heading. For each experiment, the values table lists the experiment number, ordered according to the input listing; a descriptor for the experiment, which in this case is the title record from the SDF; the type of sensitivities coefficients on the file, which are all $k_{\text {eff }}$ sensitivities in this case; the value, which in this case represents the computed $k_{\text {eff }}$ values from each SDF; the uncertainty in the computed value due to cross-sectioncovariance data, which is present because uncert is included in the parameter input; and the $c_{k}$ value of the experiment compared to the application.

In Fig. 134, the first $c_{k}$ values listed compare the ${ }^{233} \mathrm{U}$ application to HEU-MET-FAST experiments and provide $c_{k}$ values near 0.0 . These are expected results because these experiments have almost no features similar to those in the application. Sorting the results by $c_{k}$ value in descending order reveals the experiments that are most similar to the application. As shown in Fig. 135, the most similar experiments are from the U233-SOL-THERM-013 and -016 evaluations and have $c_{k}$ values very close to 1.0. However, in the output edit, the title on the SDF does not show the case number. The exact case can be identified in the Applications List edit under Input Data, or the TSUNAMI-IP parameter input usename can be input to identify the experiments and applications according to their filenames instead of the title on the SDF.

From the listed in $c_{k}$ values, it is evident that there are many experiments with high, $c_{k} \geq 0.9$, similarity to the application. To view only those experiments, use the $c_{k}$ Summary edit, shown in Fig. 136. Here there are 198 experiments with $c_{k} \geq 0.9$. Each of the experiments is listed in the output along with its $c_{k}$ value relative to the application. 


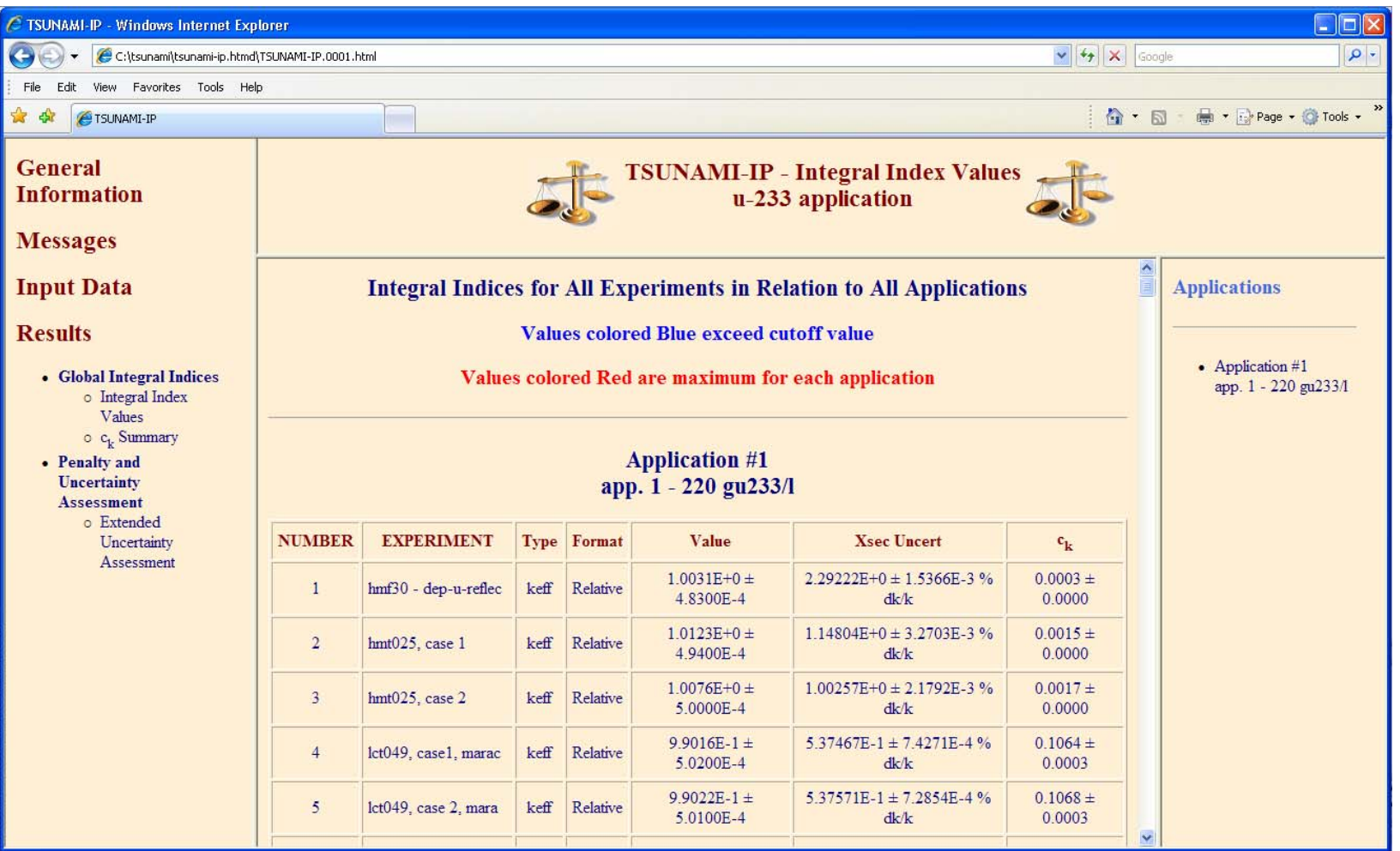

Fig. 134. TSUNAMI-IP integral index values edit in HTML output.

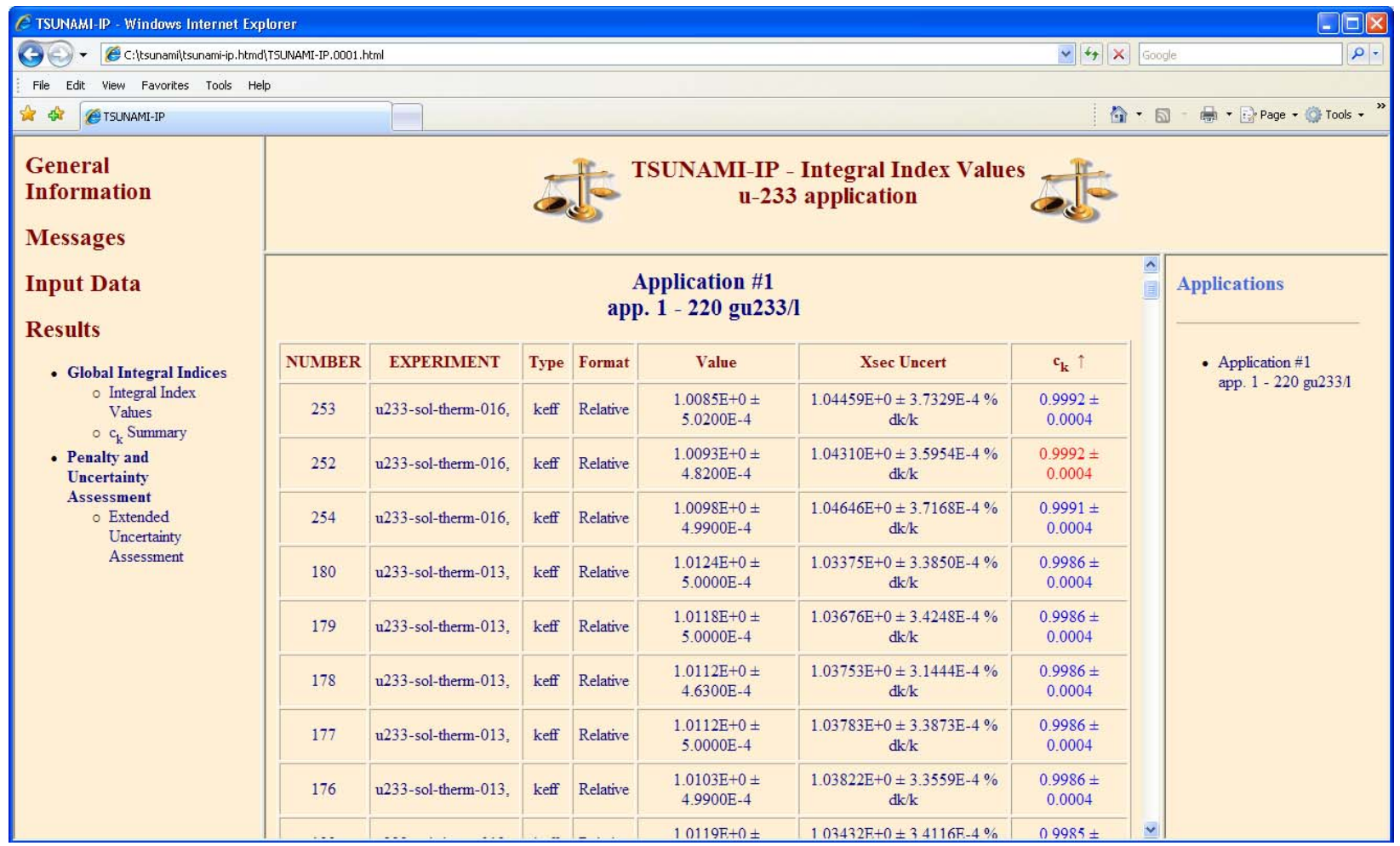

Fig. 135. TSUNAMI-IP integral index values edit in HTML output sorted by descending $c_{k}$. 


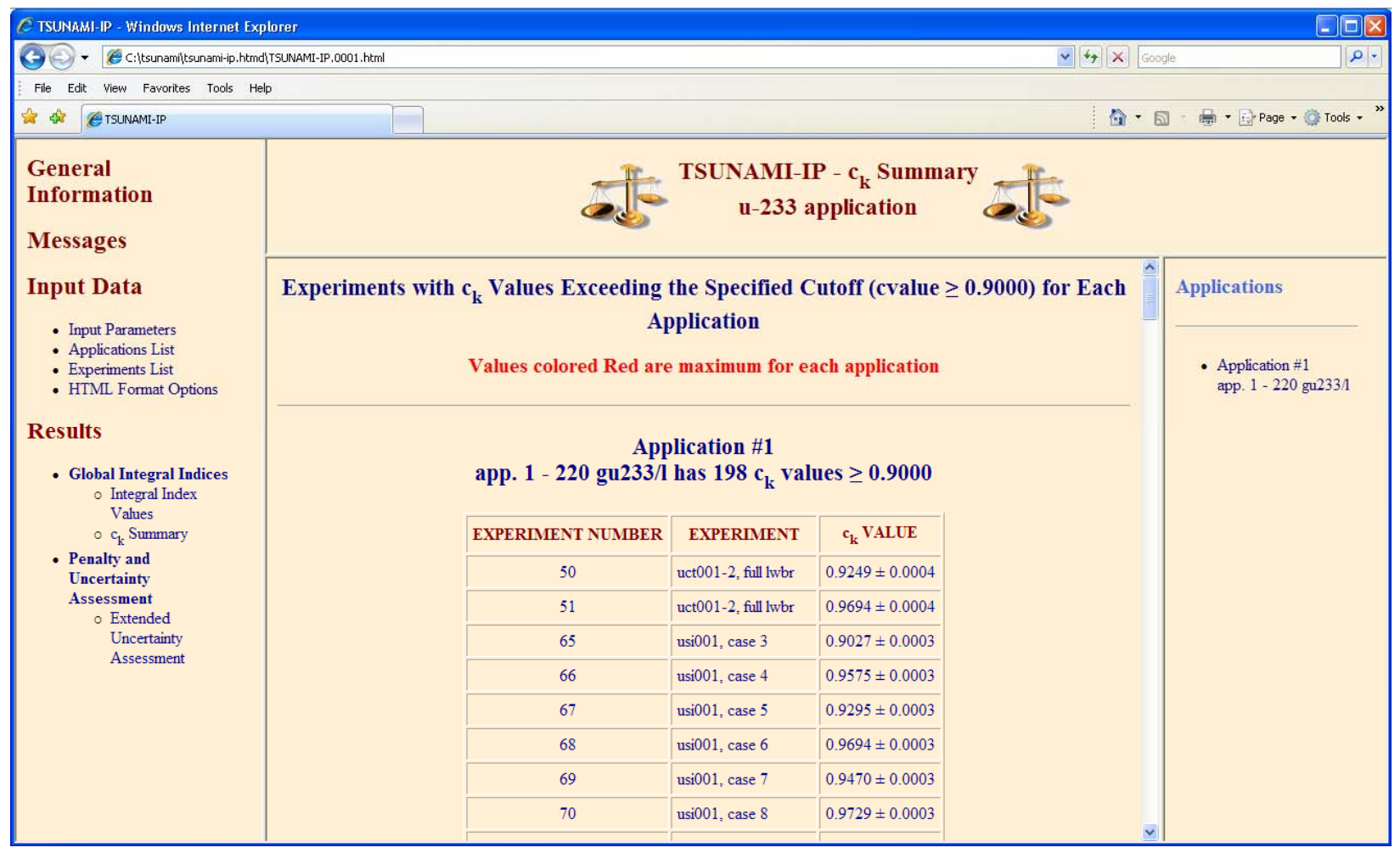

Fig. 136. TSUNAMI-IP $c_{k}$ summary edit in HTML output.

\subsection{EXAMINATION OF NUCLIDE-REACTION-SPECIFIC SIMILARITY}

Additional capabilities are available in TSUNAMI-IP to assess similarity on a nuclide-reaction-specific basis and to identify specific components of the target application that are not validated by available benchmarks. If inadequacies in the benchmark set are identified, the characteristics of experiments that meet the validation requirements of the target application can be determined from the TSUNAMI data, and optimized new experiments can be designed. ${ }^{10}$ Several options are available in TSUNAMI-IP to examine similarity or coverage in terms of sensitivity and uncertainty criteria for individual nuclidereaction pairs.

The integral index "g," referred to as "little g," determines the coverage of a nuclide reaction in terms of the amount of overlap the experiment sensitivity profile provides relative to the corresponding sensitivity profile of the application. This index is normalized between 0 and 1 , where 0 indicates that the experiment provides no coverage for the nuclide reaction, and 1 indicates that the experiment is at least as sensitive as the application in every energy group. If the sensitivity of the experiment exceeds that of the application, the little-g index will still give a value of 1 , as it does not consider overcoverage as reducing similarity. To request little $g$, enter $l g$ in the TSUNAMI-IP parameter data or in the palette dialog, as shown in Fig. 137.

The extended $c_{k}$ edit offers two means of interpreting similarity in terms of uncertainty information. First, the $c_{k}$ contribution edit details the amount of the overall $c_{k}$ between a given experiment and application from each covariance matrix. The sum of the individual contributions is the $c_{k}$ value. It is instructive to examine the $c_{k}$ contributions of the application compared to itself to see the values of $c_{k}$ contributions from an experiment with an ideal $c_{k}$ of 1.0. Any change in the $c_{k}$ contributions between an experiment and the application indicates a reduction in similarity. To facilitate the examination of the $c_{k}$ 
contributions for the application compared to itself, the first edit available in this output section is for experiment $\odot$, the application.

To provide an easier means of examining nuclide-reaction-specific coverage, the individual $c_{k}$ index was added to TSUNAMI-IP for SCALE 6 . The individual $c_{k}$ is computed with the same data as $c_{k}$, but it is independently normalized between -1 and 1 for each nuclide-reaction pair. It has an advantage over the little-g index in that overcoverage by an experiment will reduce similarity as will undercoverage. It also has an advantage over the $c_{k}$ contribution in that similarity in terms of each nuclide reaction in the application for each experiment is normalized from -1 to 1 .

To request the extended $c_{k}$ edit, which includes both the $c_{k}$ contribution and the individual $c_{k}$ for each experiment compared to each application, enter $c_{-}$long in the TSUNAMI-IP parameter data, as shown in Fig. 137.

ExSITE with an updated TSUNAMI-IP input to use SDF file names in output edits, usename; compute little-g values, $1 \mathrm{~g}$; and compute $c_{k}$ contributions and individual $c_{k}$ values, $c_{-}$long, is shown in Fig. 138 . Save this input file and execute SCALE to compute the new results.

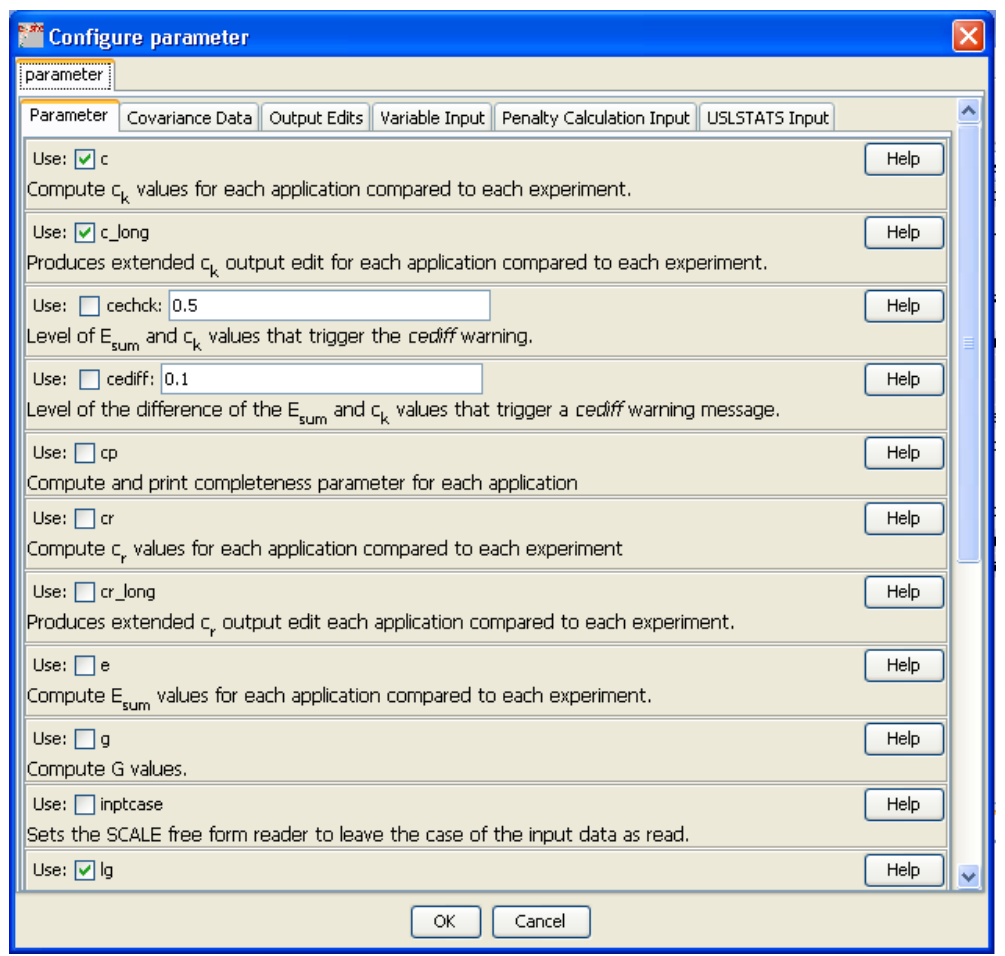

Fig. 137. ExSITE TSUNAMI-IP parameter palette dialog with selections for nuclide-reaction-specific similarity. 


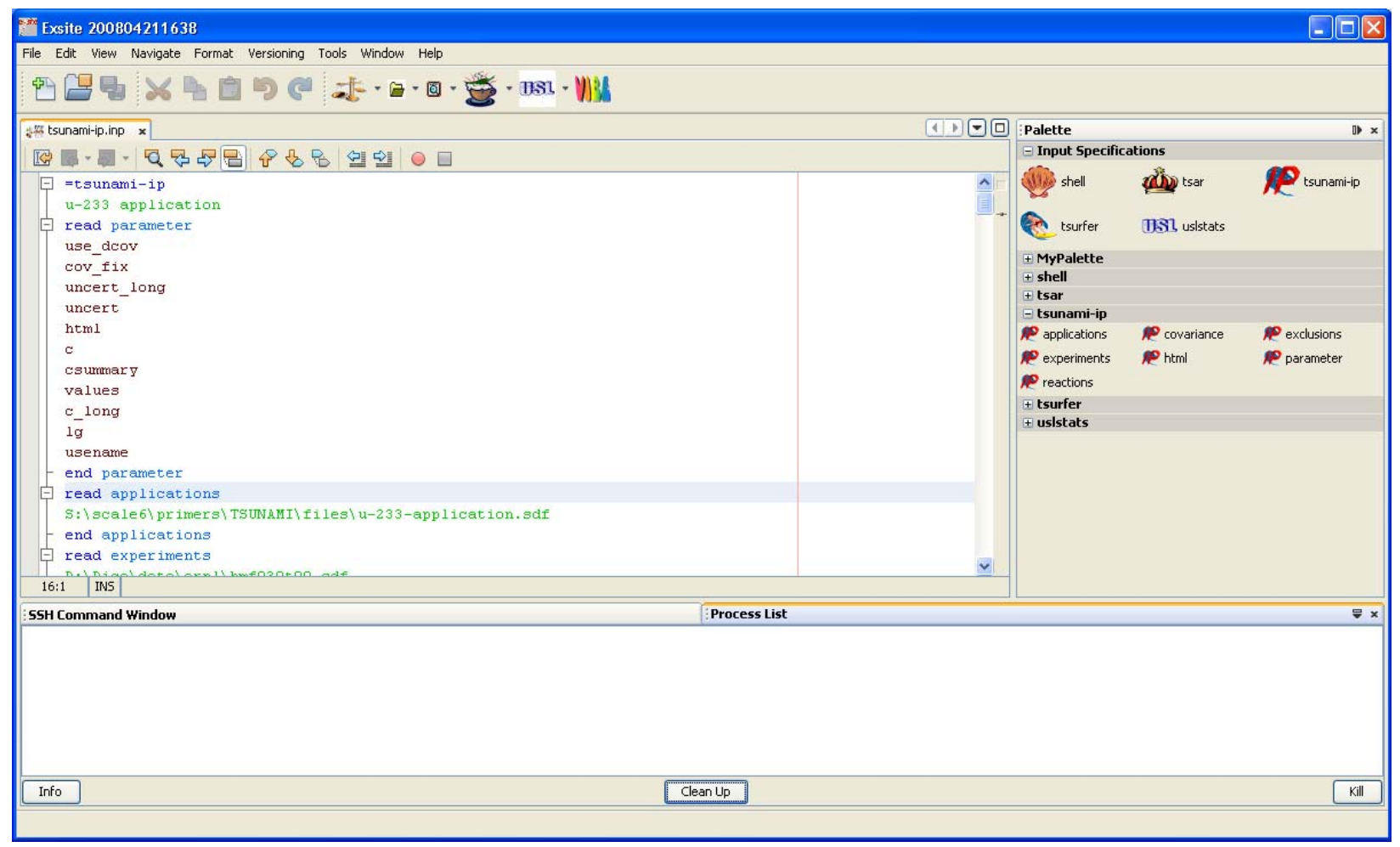

Fig. 138. ExSITE with TSUNAMI-IP input updated to include nuclide-reaction-specific similarity assessments.

\subsubsection{Little $\mathrm{g}$}

Refreshing the HTML output after the job completes reveals a new submenu in the Results section, Nuclide-Reaction-Specific Integral Indices. This submenu contains two edits, one for Nuclide-Reaction-Specific Integral Index Values, which contains little $\mathrm{g}$, and Extended $c_{k}$ Edit, which contains the $c_{k}$ contribution and individual $c_{k}$ values. Examining the Nuclide-Reaction-Specific Integral Index Values reveals the edit shown in Fig. 139. Here the little-g values are shown for all nuclides with fission, capture, or scatter sensitivities exceeding 0.01 in magnitude in the application. The first edit is for "experiment 0 ," the application compared to itself. Here by definition all little-g values are 1. The double menu on the right side of the browser provides access to edits of all combinations of applications and experiments. The top menu provides a shortcut into the bottom menu. Clicking on an application in the top menu will set the bottom menu to that application. Selections in the bottom menu control the data that are displayed in the center pane of the browser. Selecting the data for "Experiment \#246" produces the output shown in Fig. 140. Experiment \#246 is U233-SOL-THERM-016 case 3, which was previously examined with VIBE and Javapeño in Sect. 8.4, and has a very high $c_{k}$ value of 0.9946 relative to the ${ }^{233} \mathrm{U}$ application. For little g, sensitivity for ${ }^{1} \mathrm{H}$ scatter has the largest magnitude, and little $\mathrm{g}$ demonstrates fair coverage with a value of 0.8459. The sensitivity with the second highest magnitude is ${ }^{233} \mathrm{U}$ fission, and little $\mathrm{g}$ demonstrates excellent coverage with a value of 0.9947 . The $\sim 85 \%$ coverage of the application by the experiment for ${ }^{1} \mathrm{H}$ scatter can be visualized using Javapeño, as shown in Fig. 141, where the sensitivity of the application exceeds that of the experiment in the fast region. The inability of little $g$ to identify overcoverage is demonstrated in Fig. 142, where the experiment is more sensitive than the application, especially in the thermal region. For ${ }^{235} \mathrm{U}$ fission, the little $\mathrm{g}$ value is 0.9947 , indicating almost complete coverage. However, any bias in thermal region could be exaggerated in the experiment relative to its use in the 
application. It is conceivable that these exaggerated biases in ${ }^{233} \mathrm{U}$ fission could mask biases in other nuclide reactions in the application.

To request little-g edits for nuclides and reactions other than those shown in the Nuclide-Reaction Specific Integral Index Values edit, the lgall parameter can be used to display fission, capture, and scatter little-g values for all nuclides, or the reactions block can be used to identify nuclide reactions for further analysis with little g. These specifications will not be further reviewed here, but they are documented in the TSUNAMI-IP manual.

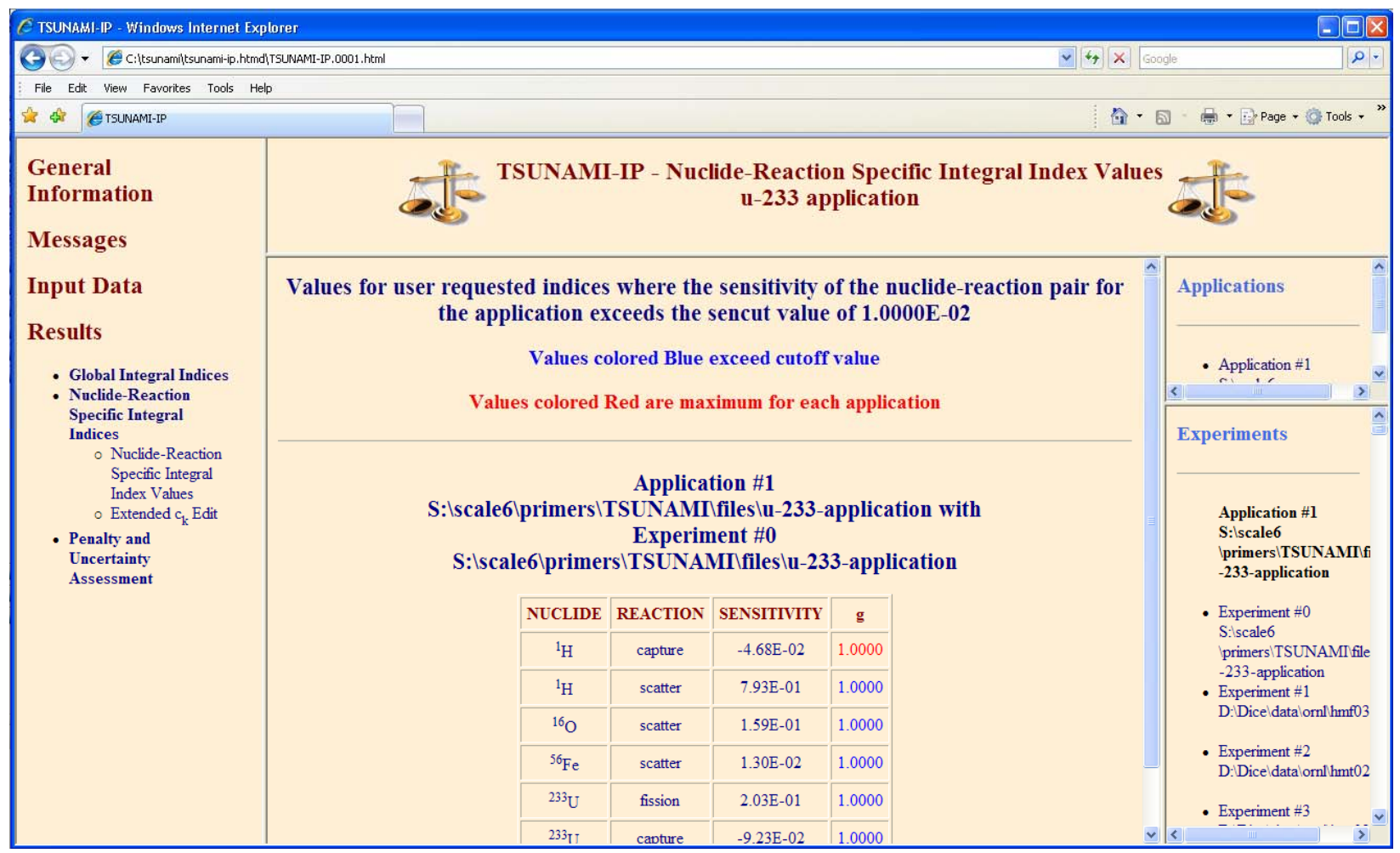

Fig. 139. TSUNAMI-IP nuclide-reaction-specific integral index values for ${ }^{233} \mathrm{U}$ application compared to itself in HTML output. 


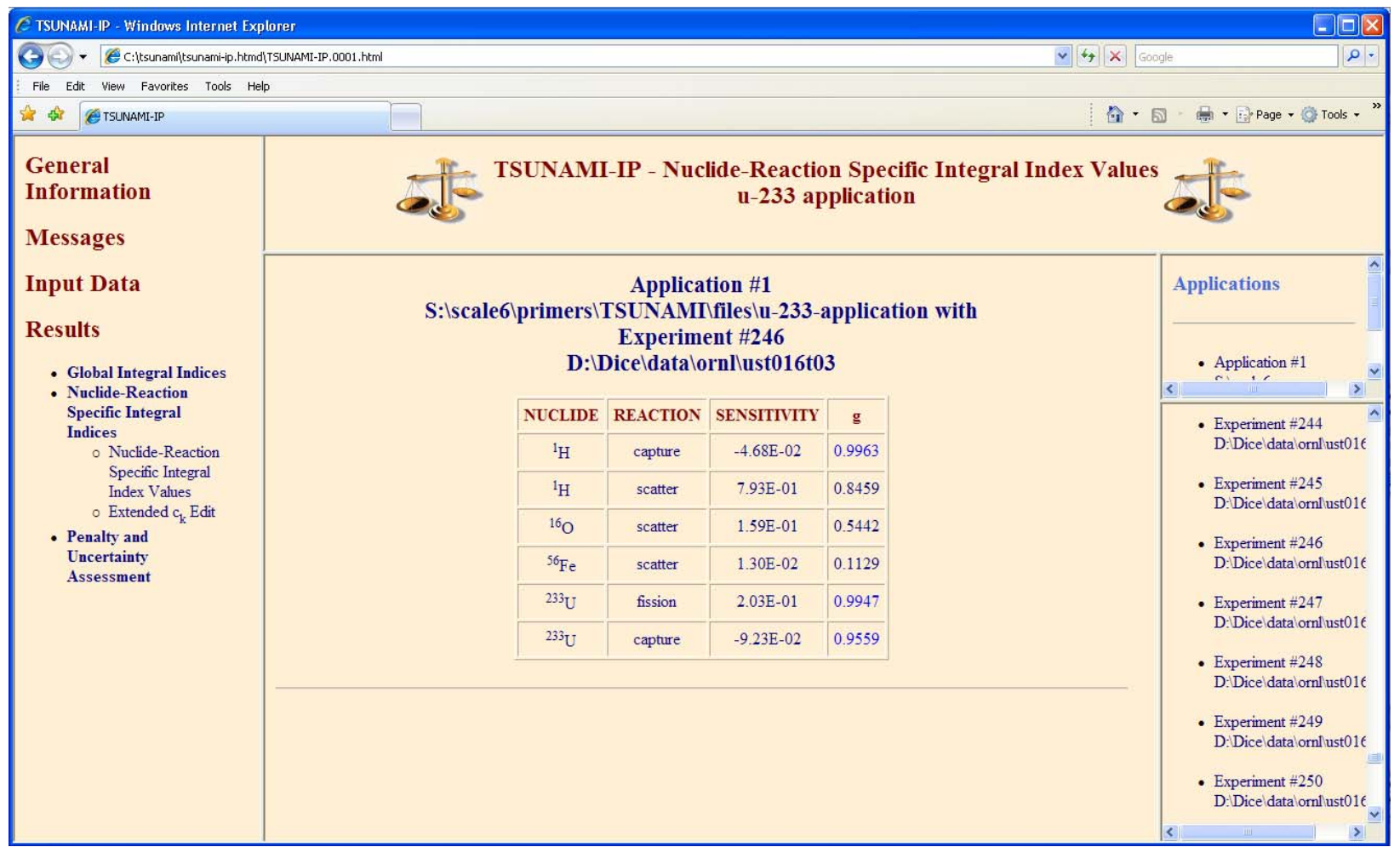

Fig. 140. TSUNAMI-IP nuclide-reaction-specific integral index values for ${ }^{233} U$ application compared to U233-SOL-THERM-016 case 3 in HTML output.

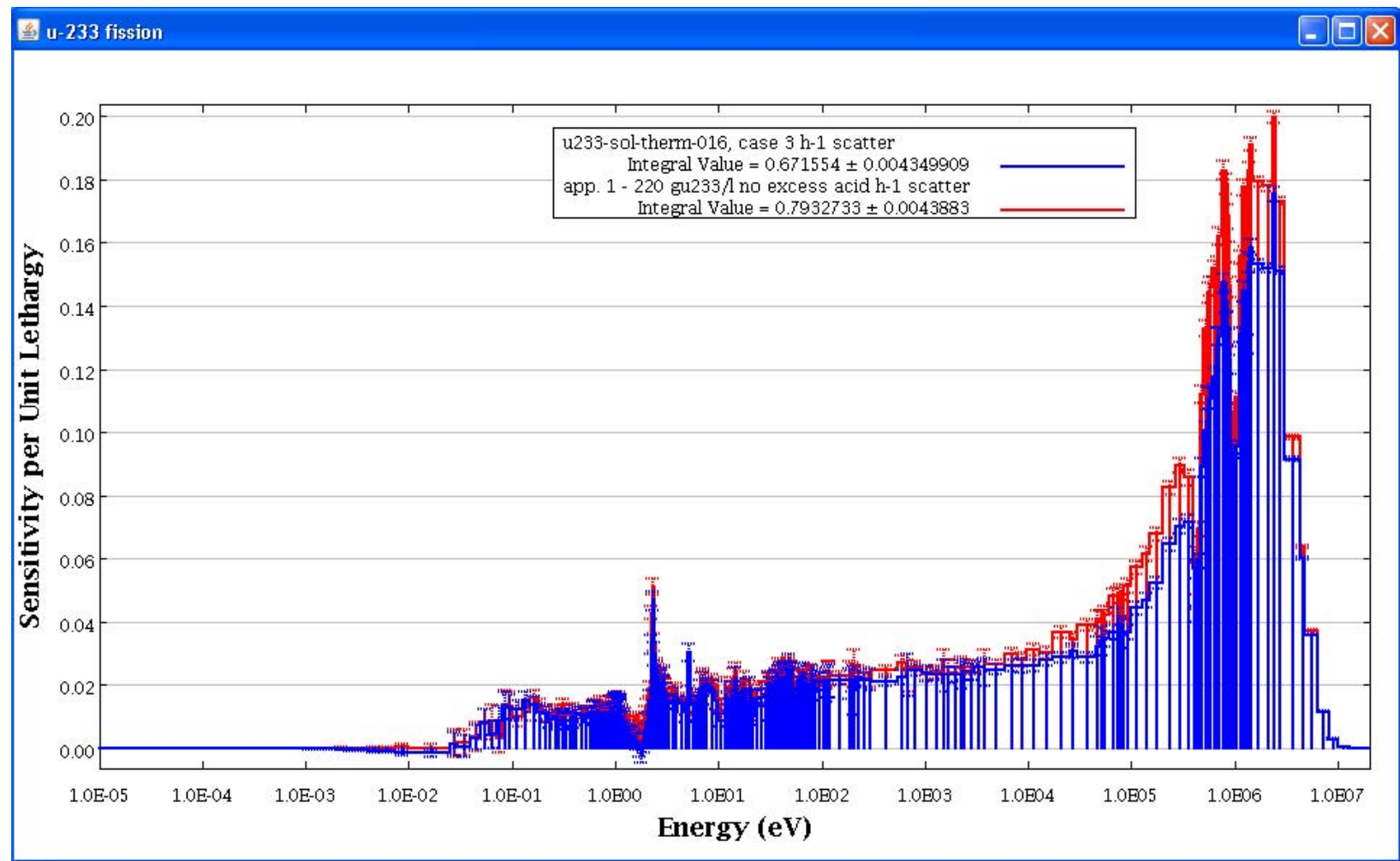

Fig. 141. Javapeño plot of ${ }^{1} \mathrm{H}$ scatter for ${ }^{233} \mathrm{U}$ application and U233-SOL-THERM-016 case 3. 


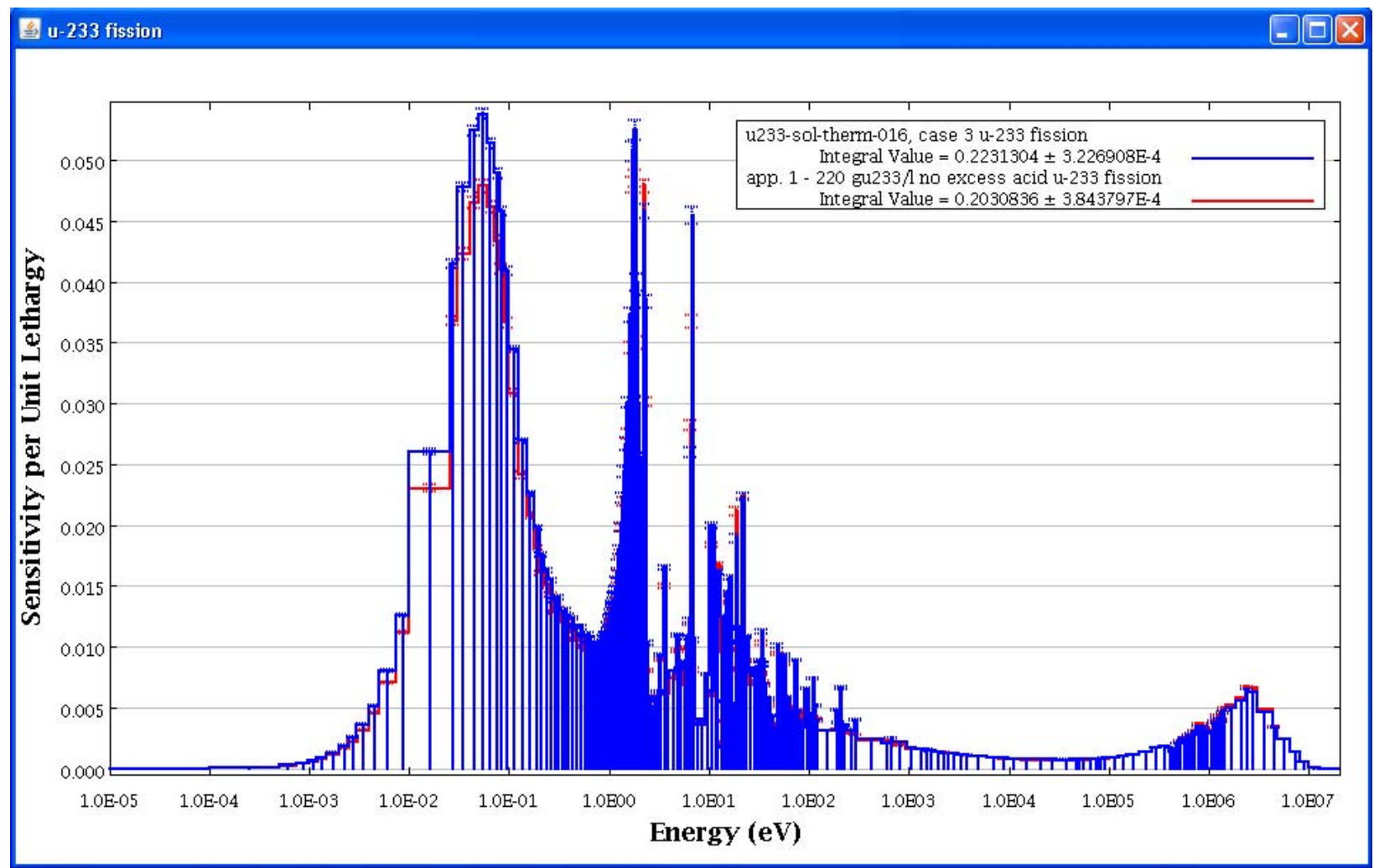

Fig. 142. Javapeño plot of ${ }^{233} \mathrm{U}$ fission for ${ }^{233} \mathrm{U}$ application and U233-SOL-THERM-016 case 3.

\subsubsection{Extended $c_{k}$}

The Extended $c_{k}$ output edit includes both the $c_{k}$ contribution and individual $c_{k}$ edits. Like the little-g edit, the extended $c_{k}$ values are controlled with a double menu on the right side of the browser. Also like the little-g edit, Experiment $\# \odot$ provides the values for the application compared to itself, as shown in Fig. 143. Here, as in the extended uncertainty edit, the values are listed by covariance matrix in order of descending magnitude of $c_{k}$ contribution. Because the $c_{k}$ values are based on uncertainties, where the experiment is compared to itself, the extended $c_{k}$ table will be in the same order as the extended uncertainty edit. In the $c_{k}$ contributions edit, the individual values sum to the $c_{k}$ value. In the case of the application compared to itself, the $c_{k}$ value, and thus the sum of the $c_{k}$ contributions, is 1.0.

The individual $c_{k}$ values for the application compared to itself are all, by definition, 1.0. However, there are no individual $c_{k}$ values for the cross-reaction or cross-nuclide-covariance data, such as ${ }^{233} \mathrm{U}$ elastic to ${ }^{233} \mathrm{U} n, \mathrm{n}^{\prime}$, shown in Fig. 143. Also note that these cross-covariance matrices often produce negative $c_{k}$ contributions due to the shared uncertainty of two reactions. The common uncertainty can be double counted unless the cross-covariance matrices are used to remove the duplication of uncertainty.

Examining the extended $c_{k}$ edit for the ${ }^{233} \mathrm{U}$ application compared to U233-SOL-THERM-016 case 3, shown in Fig. 144, it can be seen that the top contributors to $c_{k}$ are the same as those for the application compared to itself. Also, the magnitudes of the contributions are very similar to those of the application compared to itself. These similar contributions to $c_{k}$ as the "ideal experiment," the application itself, lead to the high $c_{k}$ value of 0.9947. Also, the individual $c_{k}$ values are all very high at nearly 1.0. Thus, although the sensitivities differ in some energy ranges, when weighted with the covariance data including 
significant correlations between energy groups, the impact of the sensitivity differences on the uncertainty-weighted similarity assessment is minimized.

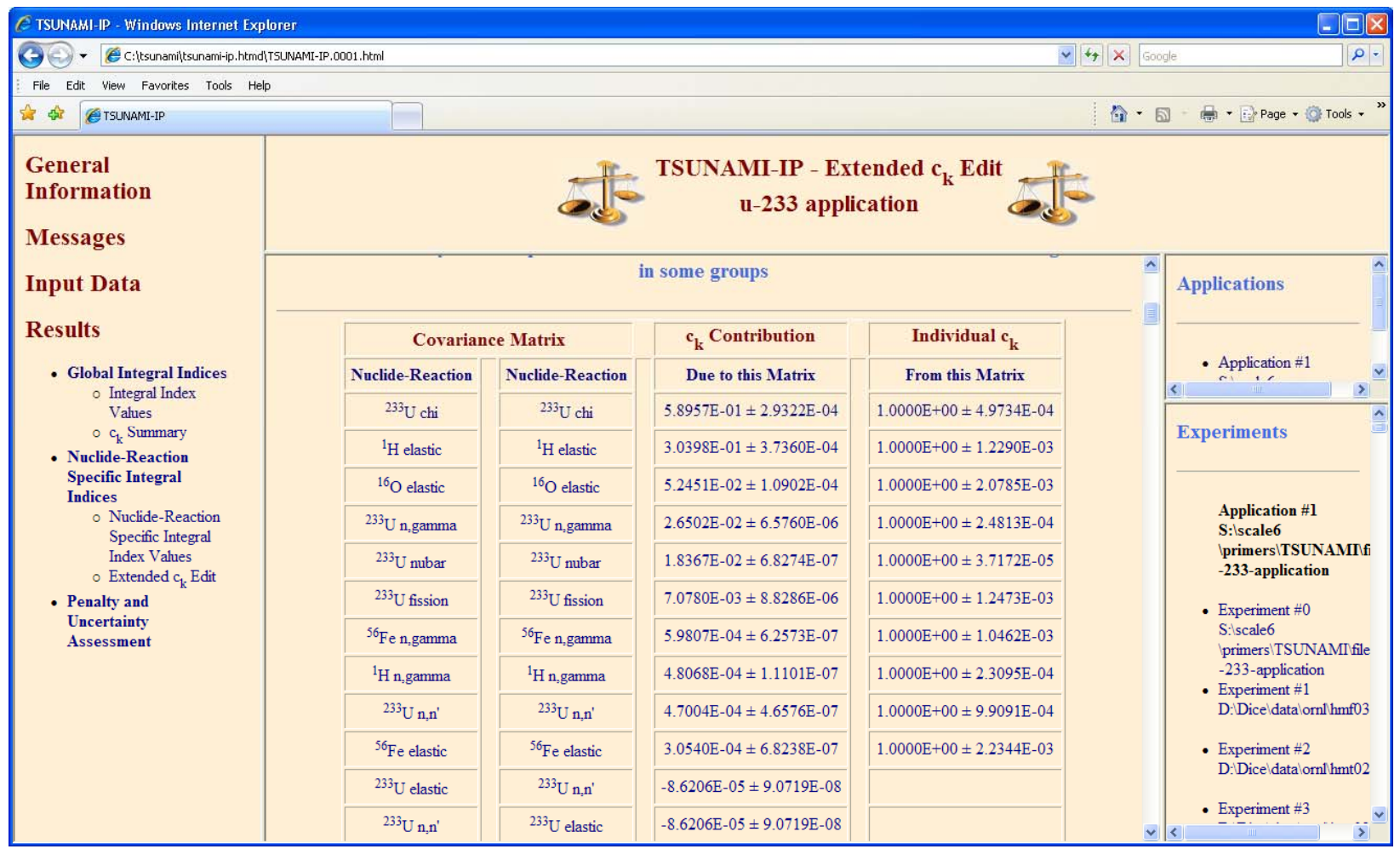

Fig. 143. TSUNAMI-IP extended $c_{k}$ edit for ${ }^{233} \mathrm{U}$ application compared to itself in HTML output. 


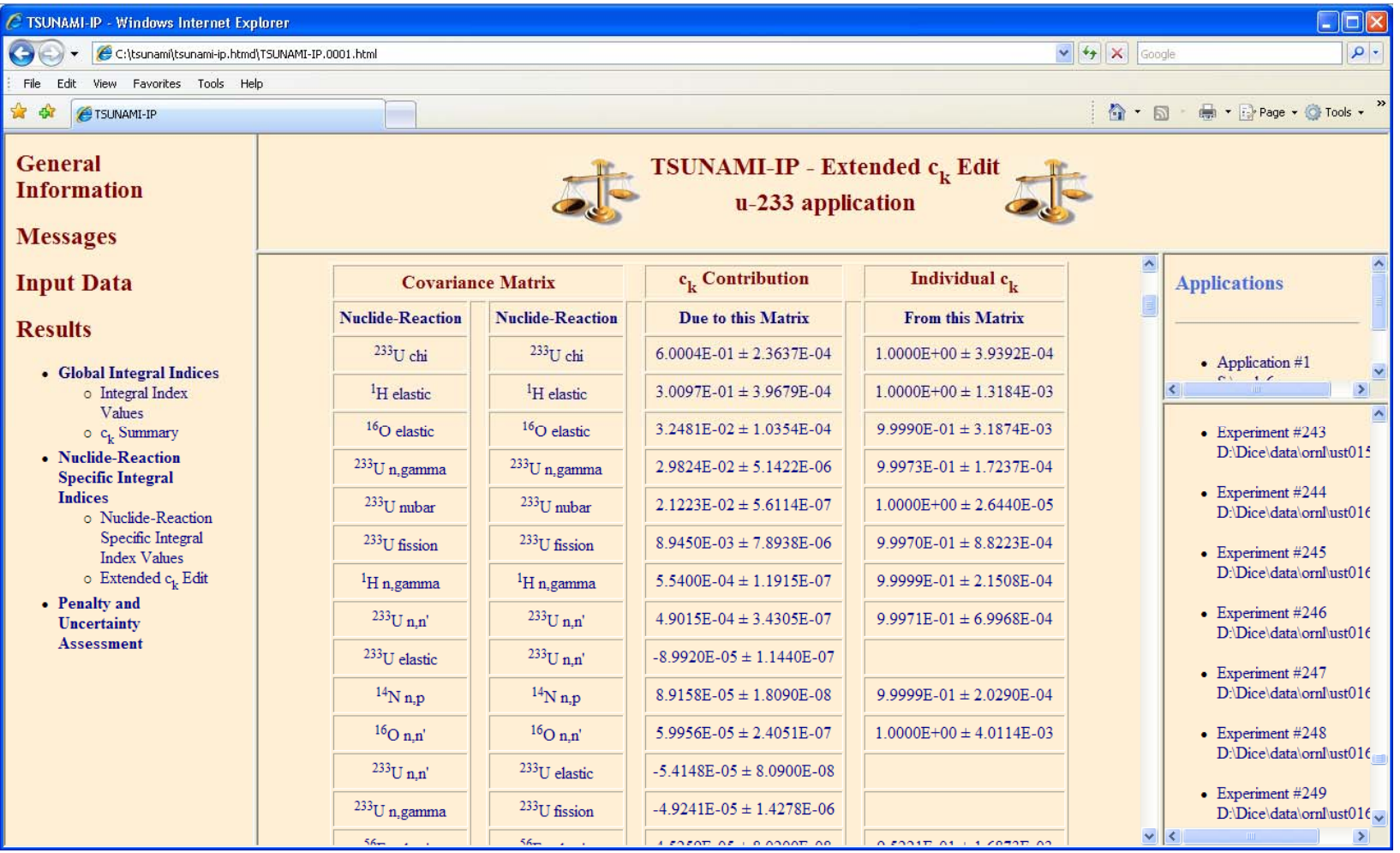

Fig. 144. TSUNAMI-IP extended $c_{k}$ edit for ${ }^{233} \mathrm{U}$ application compared to U233-SOL-THERM-016 case 3 in HTML output. 



\section{BIAS ASSESSMENT WITH $c_{k}$ TRENDING}

The bias and bias uncertainty for the ${ }^{233} \mathrm{U}$ application can be determined through a trending of $c_{k}$ values using the statistical techniques documented in NUREG/CR-6698, Guide for Validation of Nuclear Criticality Safety Calculational Methodology ${ }^{11}$ and the computer code USLSTATS ${ }^{12}$ Because the $c_{k}$ measures the similarity of a benchmark experiment to an individual application, a separate trending analysis must be conducted for each application of interest. This differs from the trending techniques presented in NUREG/CR-6698, where the bias and bias uncertainty are determined as a function of the specified trending parameter, and then the bias and uncertainty functions are evaluated at the value of the trending parameter corresponding to each design system that falls within the range of applicability of that parameter. Due to the definitions and normalizations of $c_{k}$, when used as a trending parameter, the evaluation of the bias and uncertainty will always occur at the trending parameter value of 1.0, which corresponds to the application. Furthermore, all benchmark experiments will have a $c_{k}$ value $<1.0$ in relation to the application. Thus, the evaluation of the bias and uncertainty will always require some degree of extrapolation outside the range of the trending parameters. The evaluation of the computation bias is achieved through linear regression of the $k_{\text {eff }}$ values, but the uncertainty in the bias is a nonlinear function whose value will increase as the extrapolation from the experimental data increases.

\subsection{USLSTATS TRENDING ANALYSIS}

A new interactive version of USLSTATS was released with SCALE 6. USLSTATS 6 supports a new input format, similar to other SCALE modules, and TSUNAMI-IP can generate USLSTATS input files for subsequent trending analysis. A discussion of the methodology of USLSTATS is beyond the scope of this document but can be found in NUREG/CR-6698.

\subsubsection{Generating USLSTATS Input Files with TSUNAMI-IP}

To request TSUNAMI-IP to generate a USLSTATS input file for each application using the $c_{k}$ values for all experiments, enter the keyword uslstats in the parameter data. To include only experiments with $c_{k}$ values exceeding the "cvalue" threshold criterion, enter uslsummary. These parameters are available in the ExSITE palette dialog for TSUNAMI-IP input under the USLSTATS Input subtab, as shown in Fig. 145.

Alternatively, the auto-completion feature of ExSITE can be used to assist with the input preparation. Pressing Ctrl-space while typing in the input file will activate the auto-completion menu. As shown in Fig. 146, when $u$ is entered in the text file, all valid input parameters that begin with $u$ will be listed in the pop-up menu. Using the arrow keys to navigate through the dialog will present additional information about each keyword. To use the selected keyword in the input, press Enter when the keyword is selected.

Once uslstats and uslsummary are added to the input file, run the case again. Where the TSUNAMI-IP input file is tsunami-ip.inp, the USLSTATS input files are stored in a new directory called tsunami-ip.uslstats, as shown in Fig. 147. Two USLSTATS input files were generated in this calculation. The file tsunami-ip_0001_ck_0001.usl contains all $c_{k}$ values relative to the application. If there was a second application in this analysis, a second file called tsunamiip_0002_ck_0001.usl would be generated to trend the $c_{k}$ values for the all experiments relative to the second application. The USLSTATS input file generated with only $c_{k}$ values exceeding the "cvalue" criterion is named tsunami-ip_0001_ck_sum_0001.usl. 


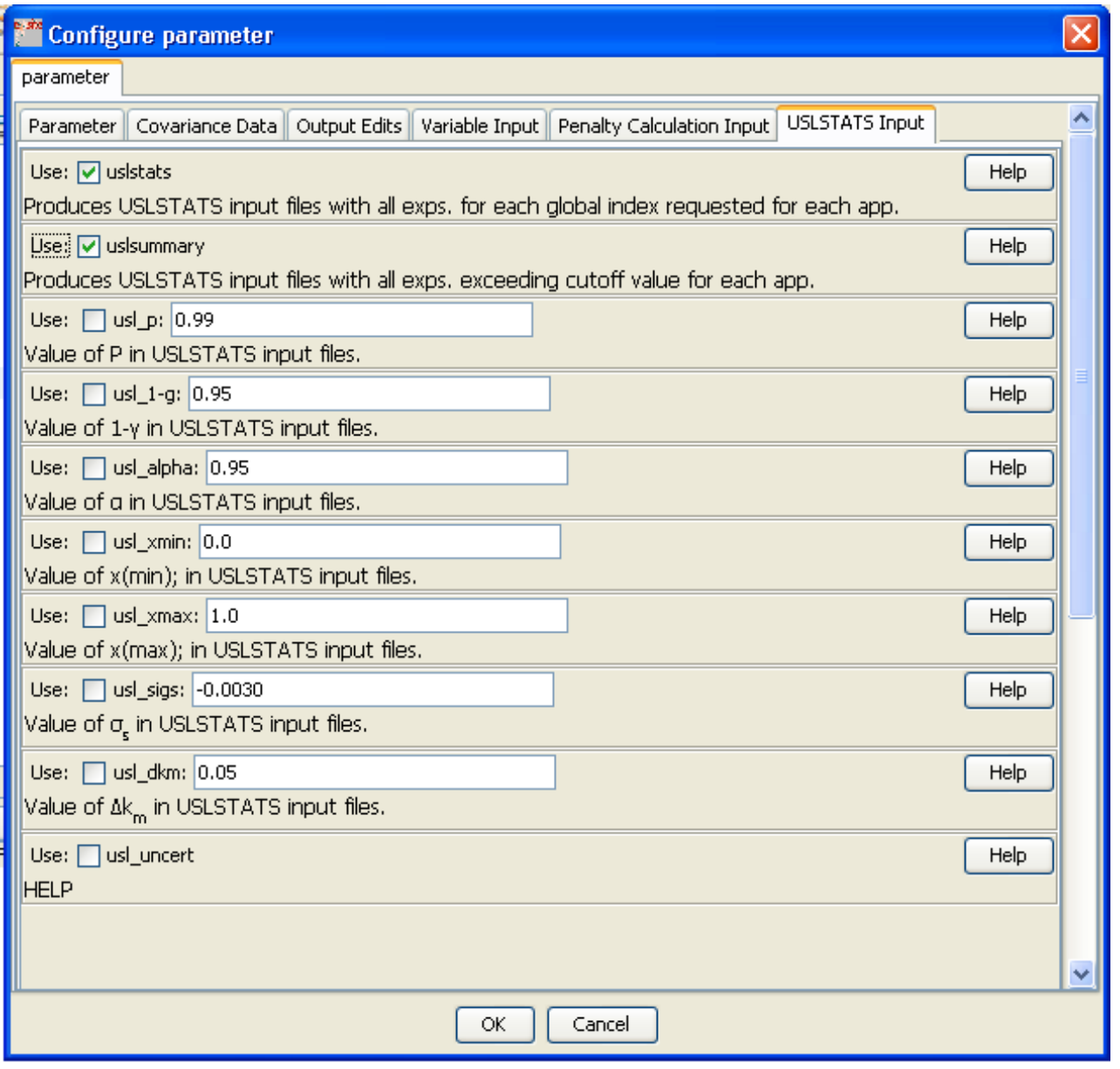

Fig. 145. ExSITE palette dialog for TSUNAMI-IP USLSTATS input parameters. 


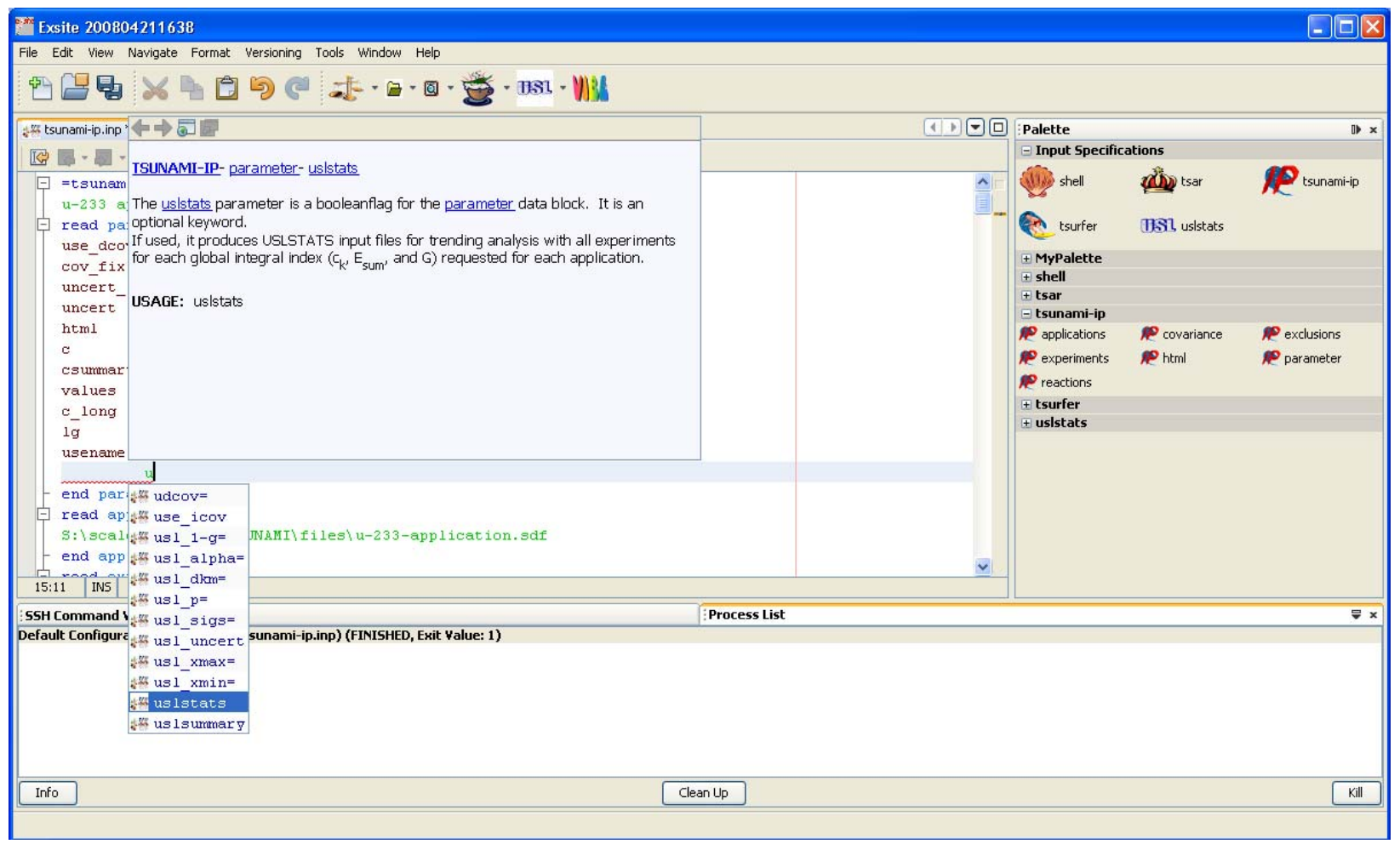

Fig. 146. ExSITE auto-completion for TSUNAMI-IP parameter data.

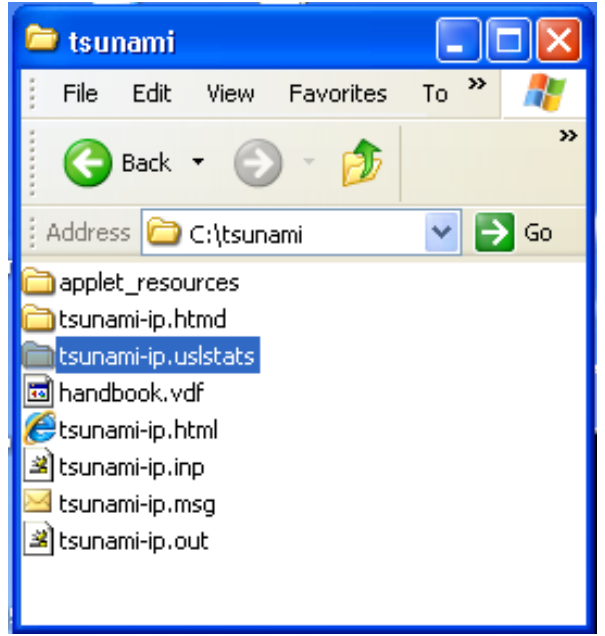

Fig. 147. Location of directory containing USLSTATS input files generated by TSUNAMI-IP. 


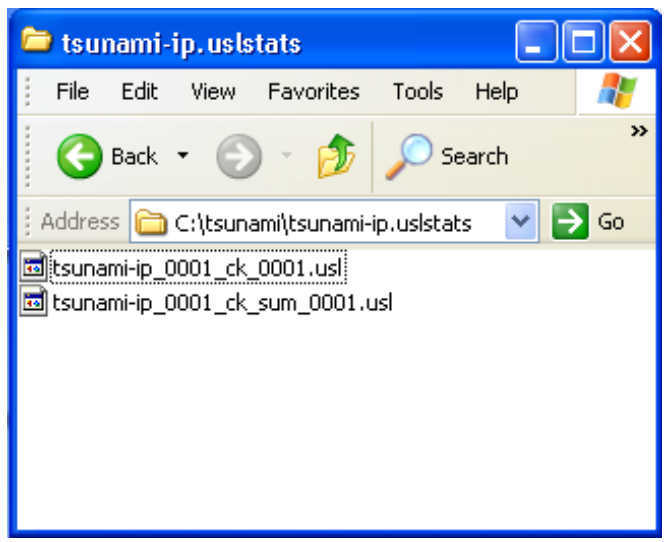

Fig. 148. USLSTATS input files generated by TSUNAMI-IP.

\subsubsection{Use of USLSTATS to Determine Bias and Bias Uncertainty Values}

USLSTATS can be opened from ExSITE with the input file preloaded by selecting the USL icon in the ExSITE toolbar, as shown in Fig. 149.

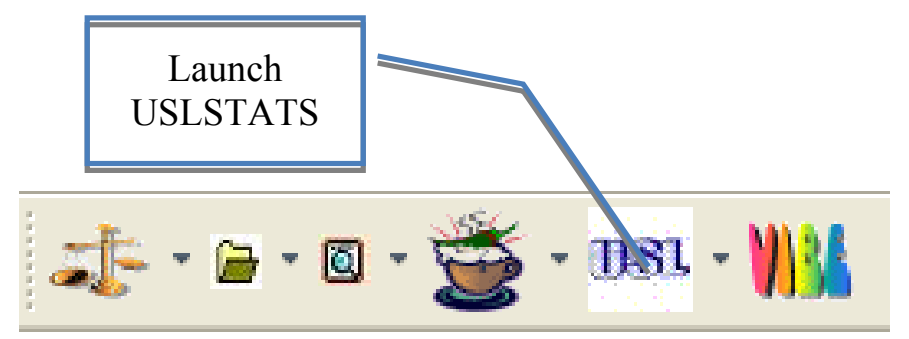

Fig. 149. ExSITE toolbar icon to launch USLSTATS.

USLSTATS will open with the two input files loaded, as shown in Fig. 150. USLSTATS uses a nested set of tabs to identify cases and the associated input and output files. The upper level of tabs is used to select the case, and the lower level of tabs is used to select the input and output files. The tsunami ip_0001_ck_sum_0001.usl file, shown in Fig. 150, uses the default settings for the parameter data. These data can be edited directly in USLSTATS, or TSUNAMI-IP can be rerun with modified parameters for USLSTATS input file generation. To execute the USLSTATS analysis using the default parameters, select the desired input file and select Analyze File from the Action menu, as shown in Fig. 151. When the calculation completes and the output loads, USLSTATS will update as shown in Fig. 152. Note that the upper tsunami-ip_0001_ck_sum_0001 tab now contains three files, the .usl input file, the .out text output, and the .html HTML output. Also, the top level of tabs now contains an additional tab for Plots. Clicking on the tab for plots will load the plot files in the applet version of Javapeño within the USLSTATS application, as shown in Fig. 153. Note that under the Plots tab, the Javapeño applet contains an additional submenu to control Javapeño. To close the data list on the right side of the plot, select Hide Reaction List from the Javapeño Window menu, as shown in Fig. 154 . 


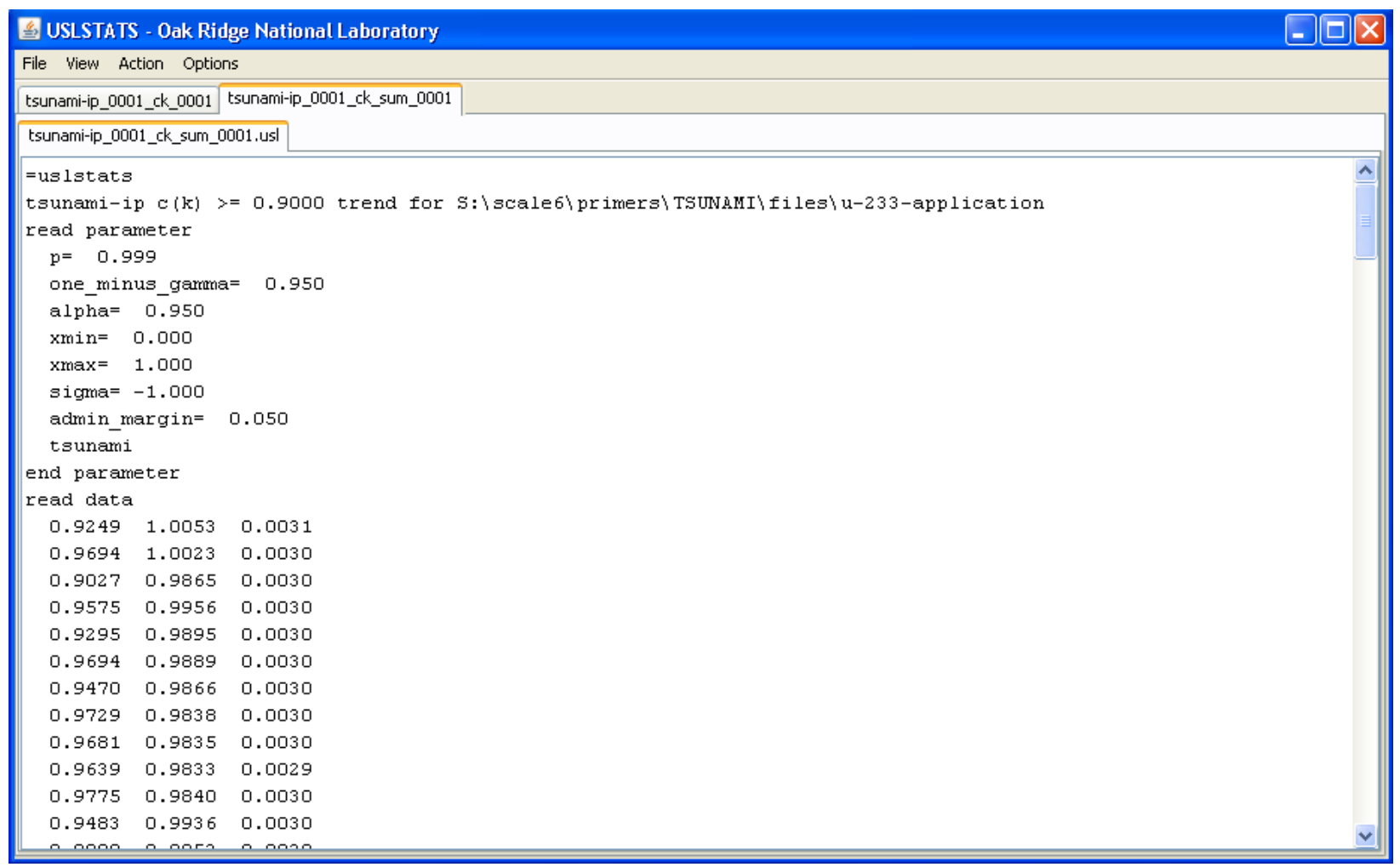

Fig. 150. USLSTATS with input files loaded. 


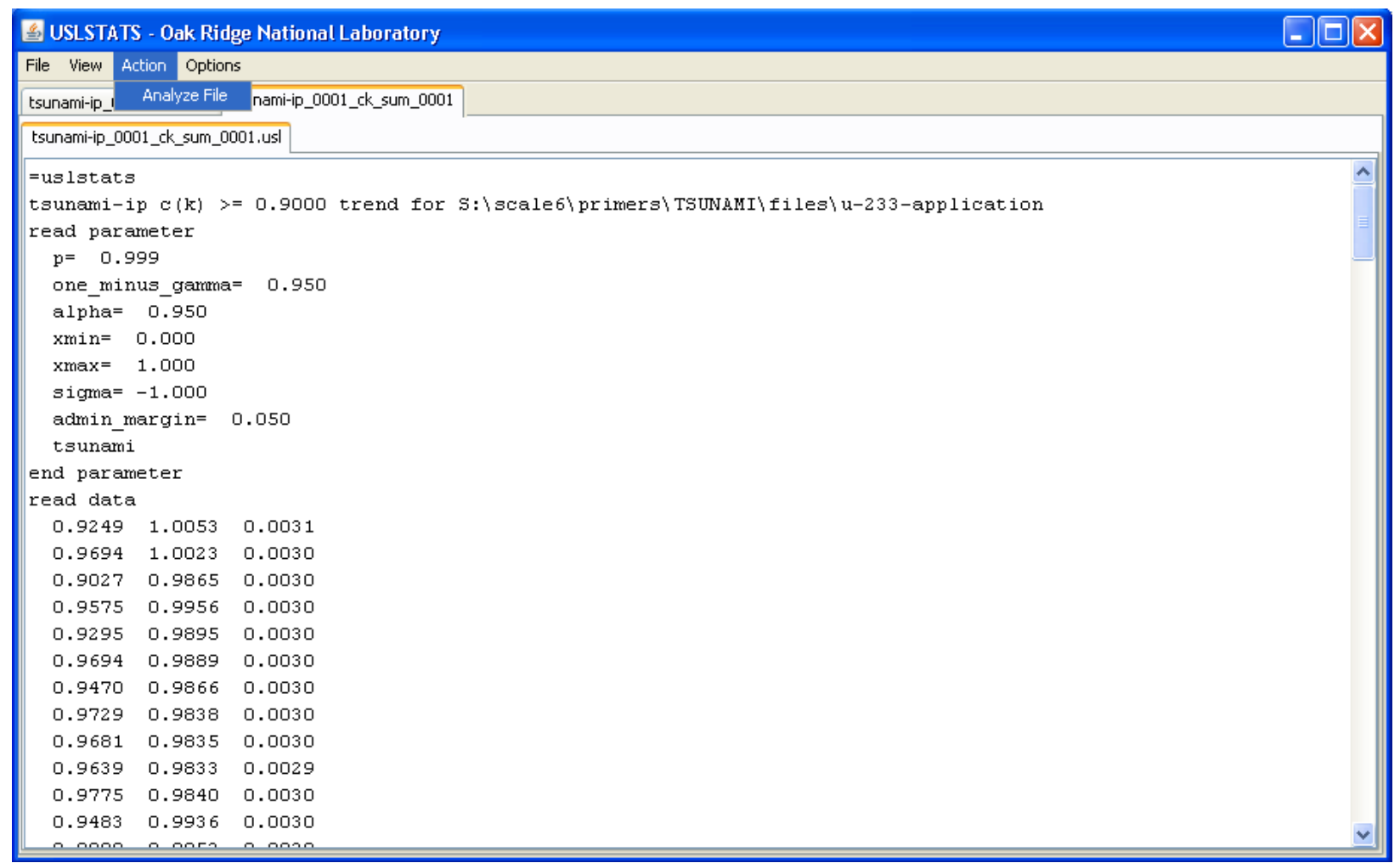

Fig. 151. USLSTATS action menu to analyze files.

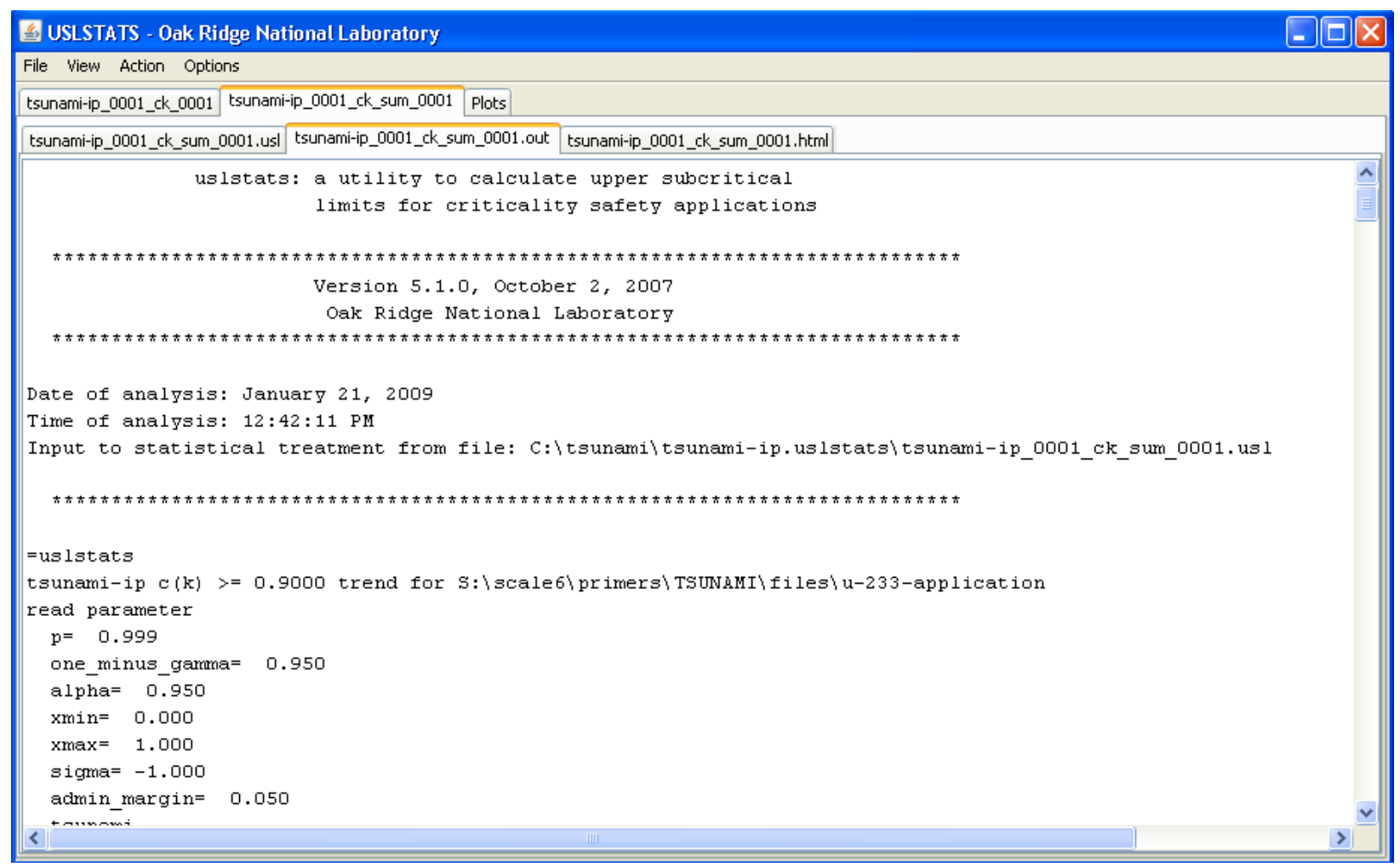

Fig. 152. USLSTATS with text output showing. 


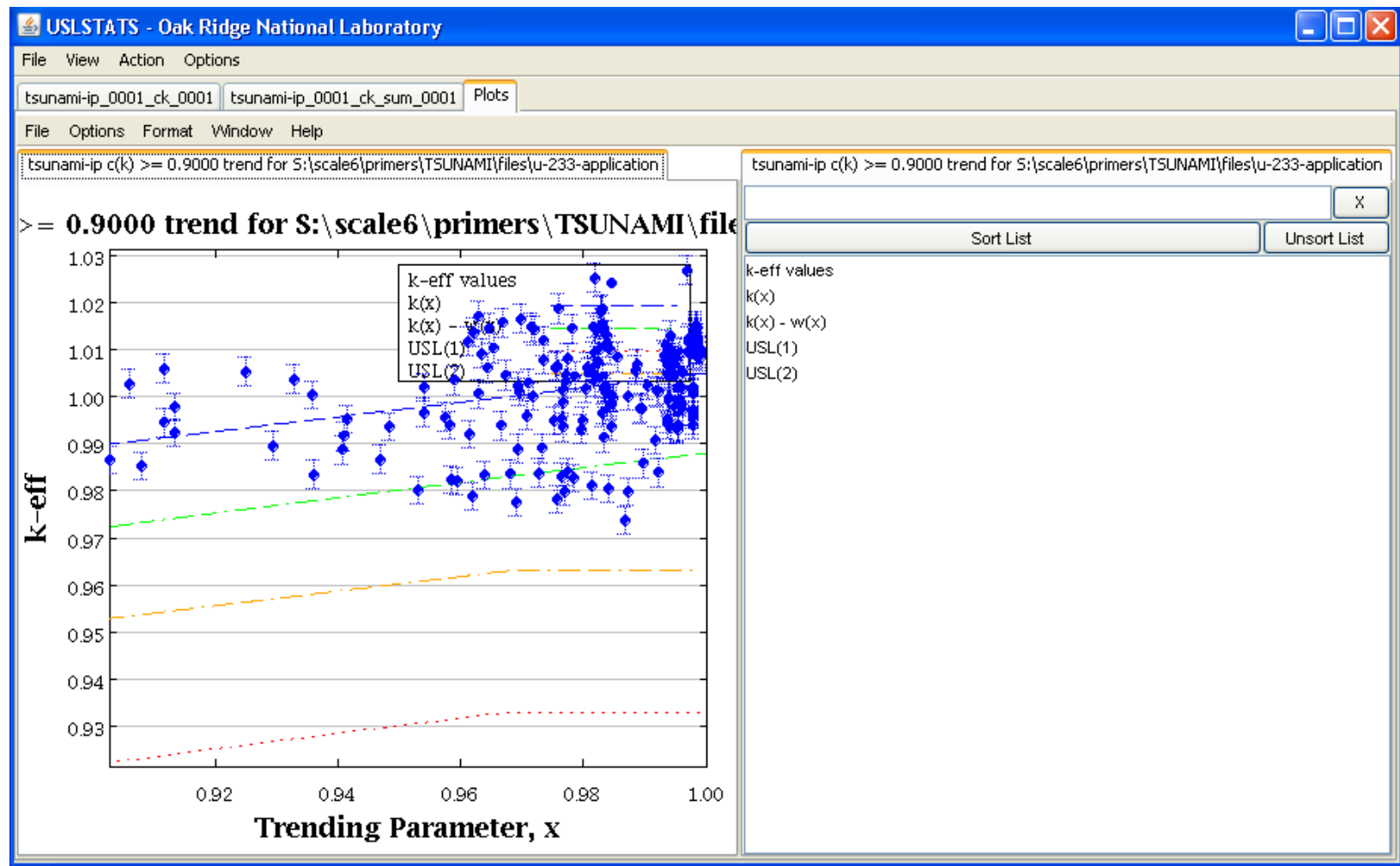

Fig. 153. USLSTATS with plot showing.

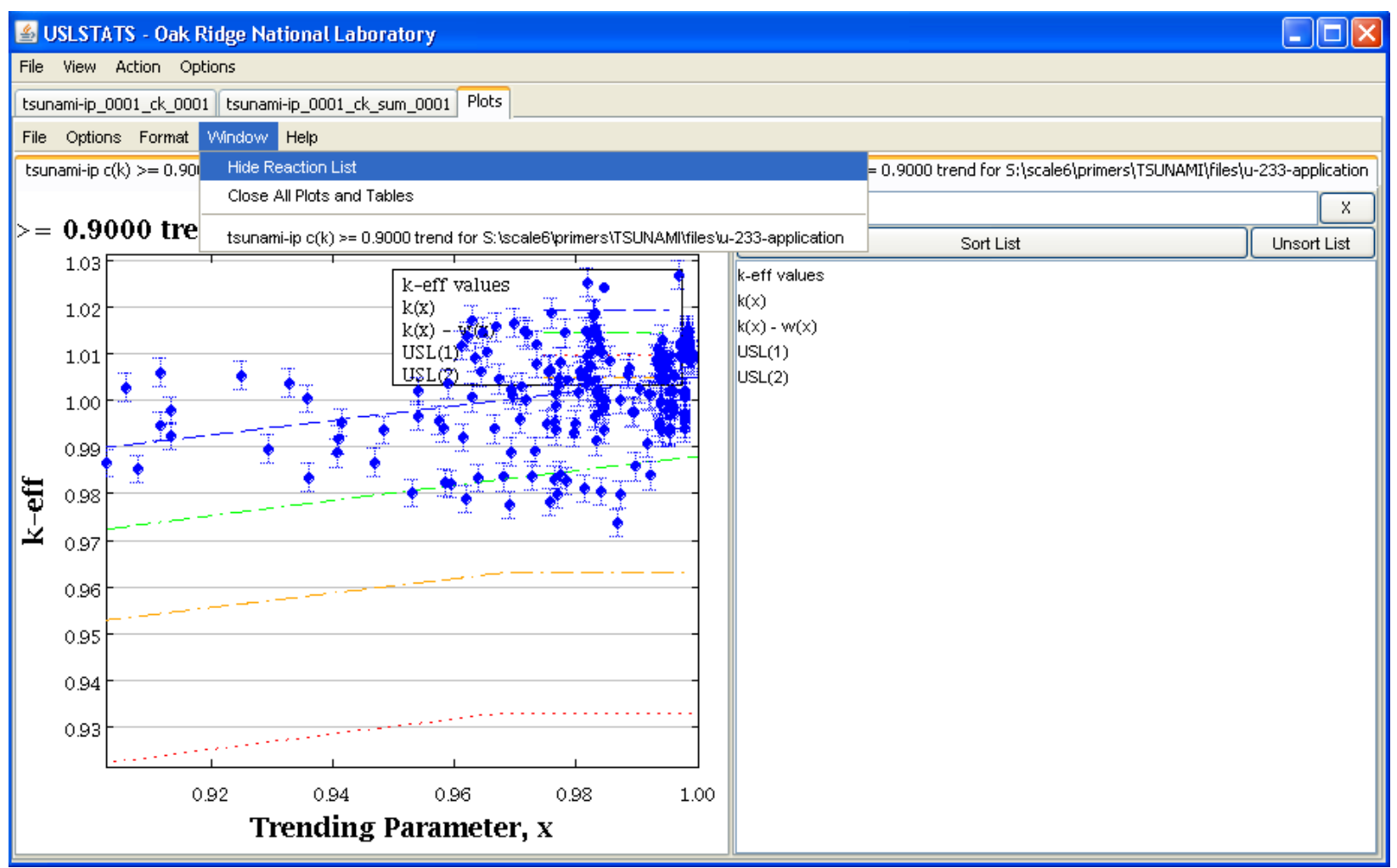

Fig. 154. USLSTATS with Javapeño applet window menu. 


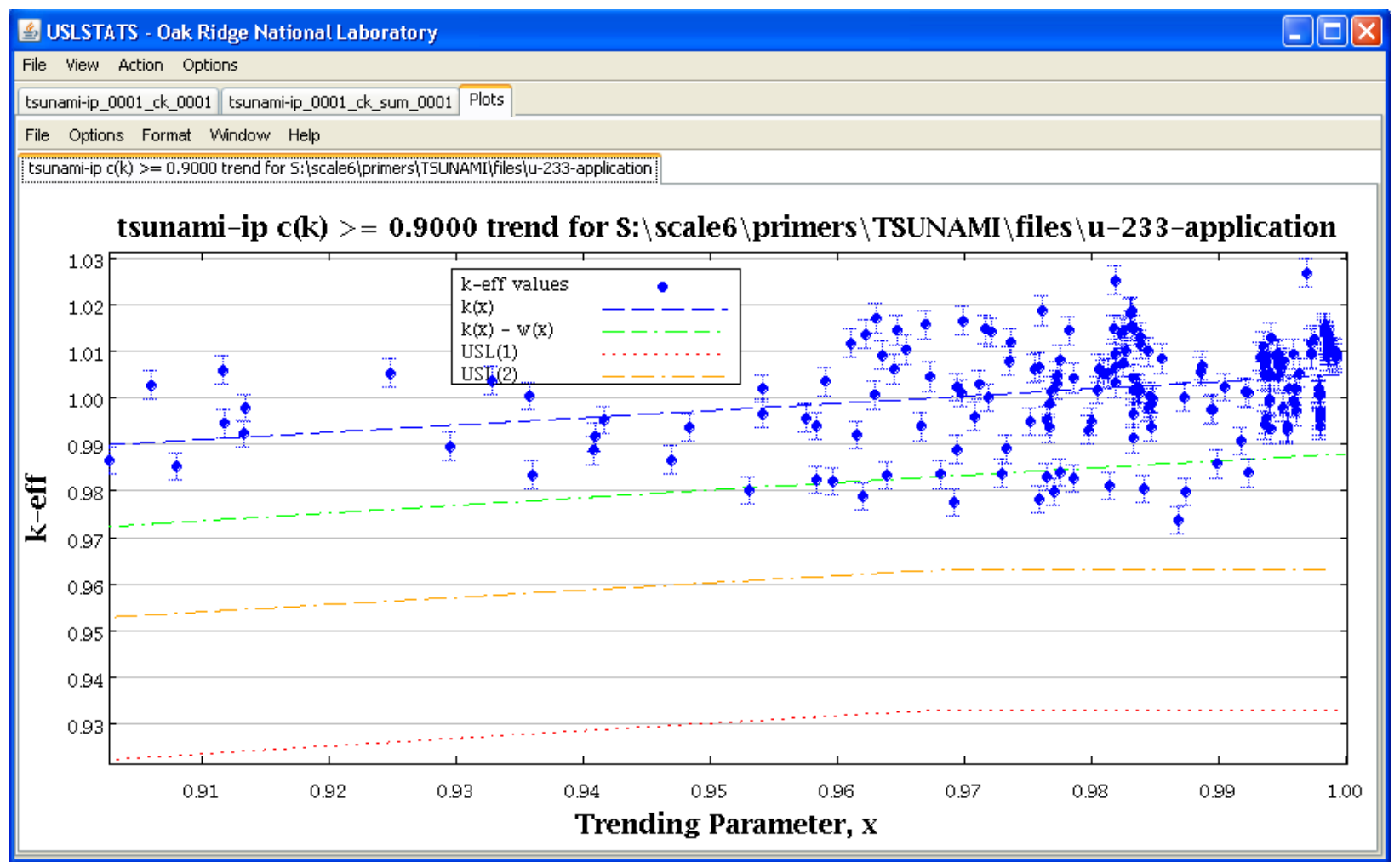

Fig. 155. USLSTATS trend plot for experiments with $c_{k} \geq 0.9$ for ${ }^{233} \mathrm{U}$ application.

The values of the computational bias and bias uncertainty can be determined from the equations for the linear fit of $k_{\text {eff, }}$ " $\mathrm{k}(\mathrm{x})$," and the quadratic bias uncertainty, "w(x)," by evaluating these functions at $\mathrm{x}=1.0$. Recall that the trending parameter, $\mathrm{x}$, is $c_{k}$ and that a $c_{k}$ value of 1.0 is the extrapolation to the application. Also, a table of values can be created using Javapeño, and the values can be read from the table where $c_{k}=1.0$. To create a table of values in Javapeño, right-click on the plot and select Create Table, as shown in Fig. 156. Extracting data from the last row of the table shown in Fig. 157, k(1.0) is the bias with a value $1.00497 \% \Delta \mathrm{k} / \mathrm{k}$. The bias less the bias uncertainty for the $95 / 95$ confidence interval requested with the default parameter data, $\mathrm{k}(1.0)-\mathrm{w}(1.0)$, is $0.987975 \% \Delta \mathrm{k} / \mathrm{k}$. The difference of these two values gives the bias uncertainty, w(1.0), with a value of $0.016995 \% \Delta \mathrm{k} / \mathrm{k}$. 


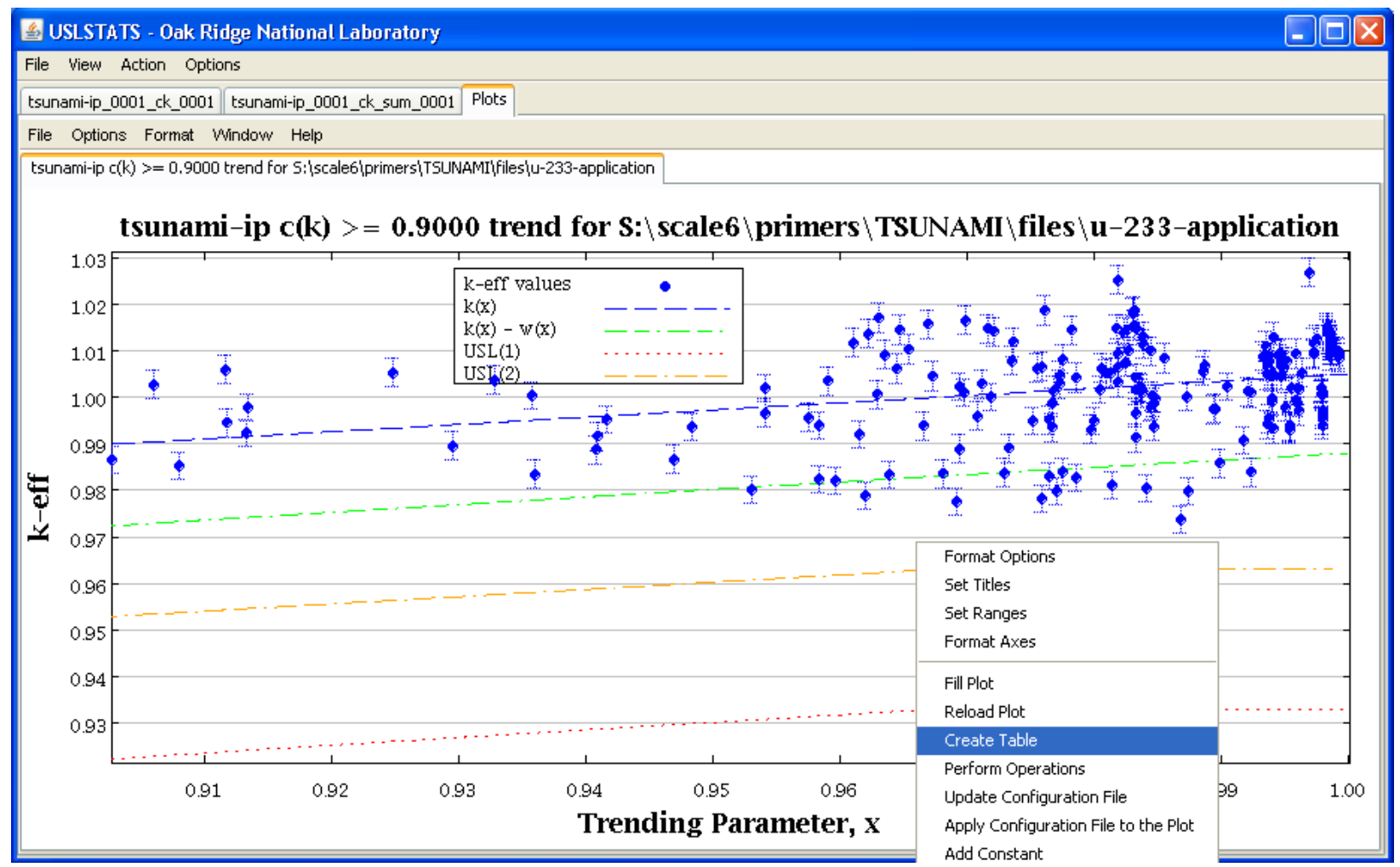

Fig. 156. USLSTATS with Javapeño pop-up menu.

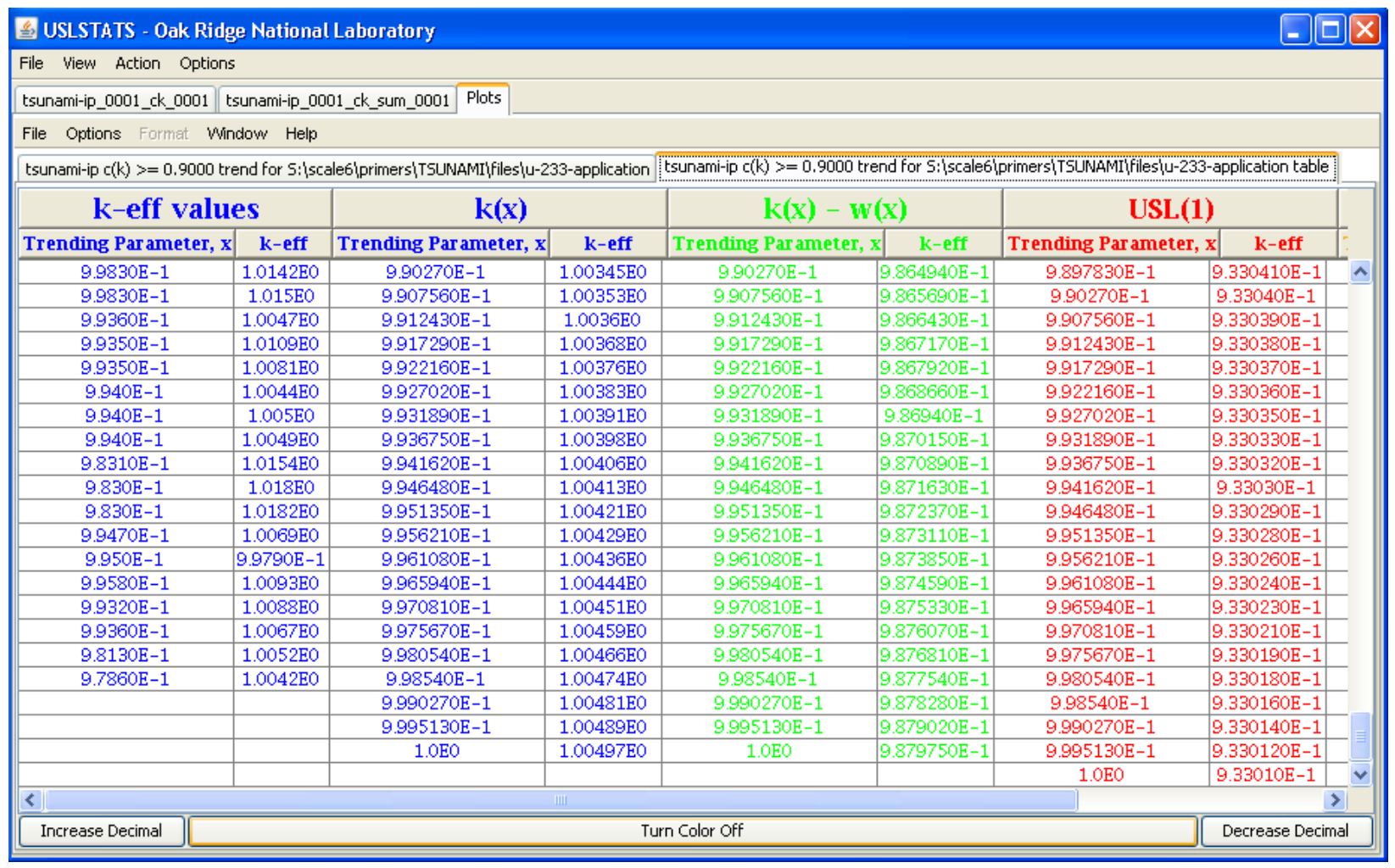

Fig. 157. USLSTATS with Javapeño table of results. 
The same procedure can be repeated for the USLSTATS input that includes all $c_{k}$ values, or the cvalue criterion can be changed in TSUNAMI-IP to include more or fewer experiments.

\subsubsection{Improving Results by Including Experimental Values}

In the preceding analysis, a few assumptions were made, which can be improved through the use of VIBE. First, the experimental $k_{\text {eff }}$ was assumed to be exactly 1.0 for all experiments. Although many experimental cases in the ICSBEP Handbook do have experiment $k_{\text {eff }}$ values of 1.0, many others have values slightly above or slightly below 1.0. Also, unless otherwise instructed, TSUNAMI-IP inputs a default experimental uncertainty of $0.3 \% \Delta \mathrm{k} / \mathrm{k}$ for each experiment. With the ability to easily query the DICE database for each experiment, these assumed values can be replaced with the actual values.

If VIBE was closed from the previous exercises, it can be easily reopened from within ExSITE by pressing the VIBE button on the toolbar, as shown in Fig. 158.

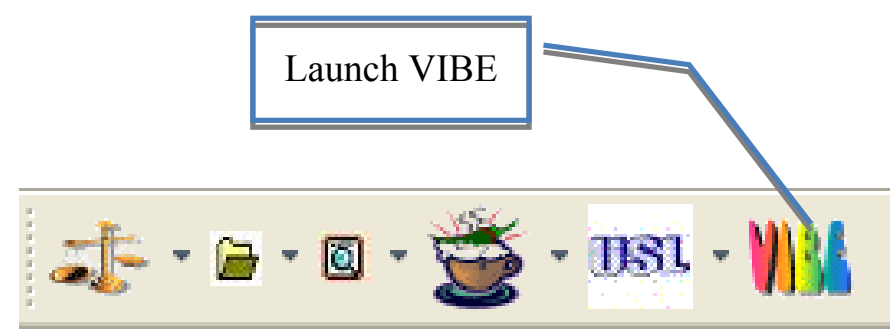

Fig. 158. ExSITE toolbar icons to launch VIBE.

Once VIBE has launched, the previously saved benchmark set for the handbook SDFs can be loaded by selecting Open benchmark set... from the File menu, as shown in Fig. 159, then browsing to the location were the handbook. vdf file was saved. In this case the vdf file was saved in C: \t sunami. After selecting the file, as shown in Fig. 160, click Open.

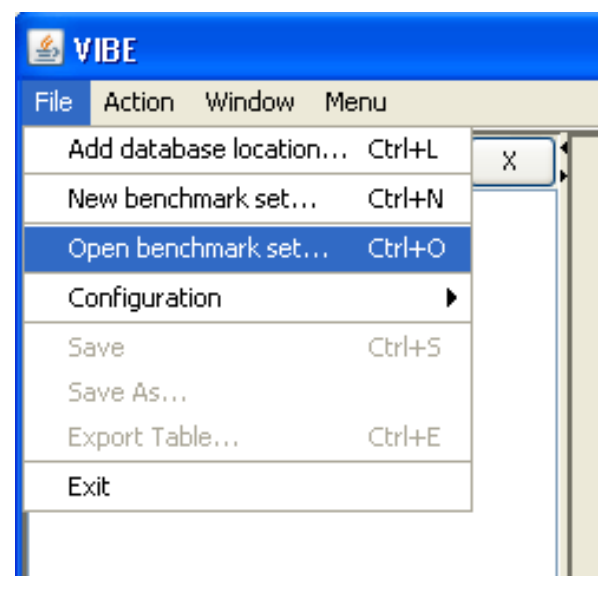

Fig. 159. VIBE file menu item to open an existing benchmark set. 


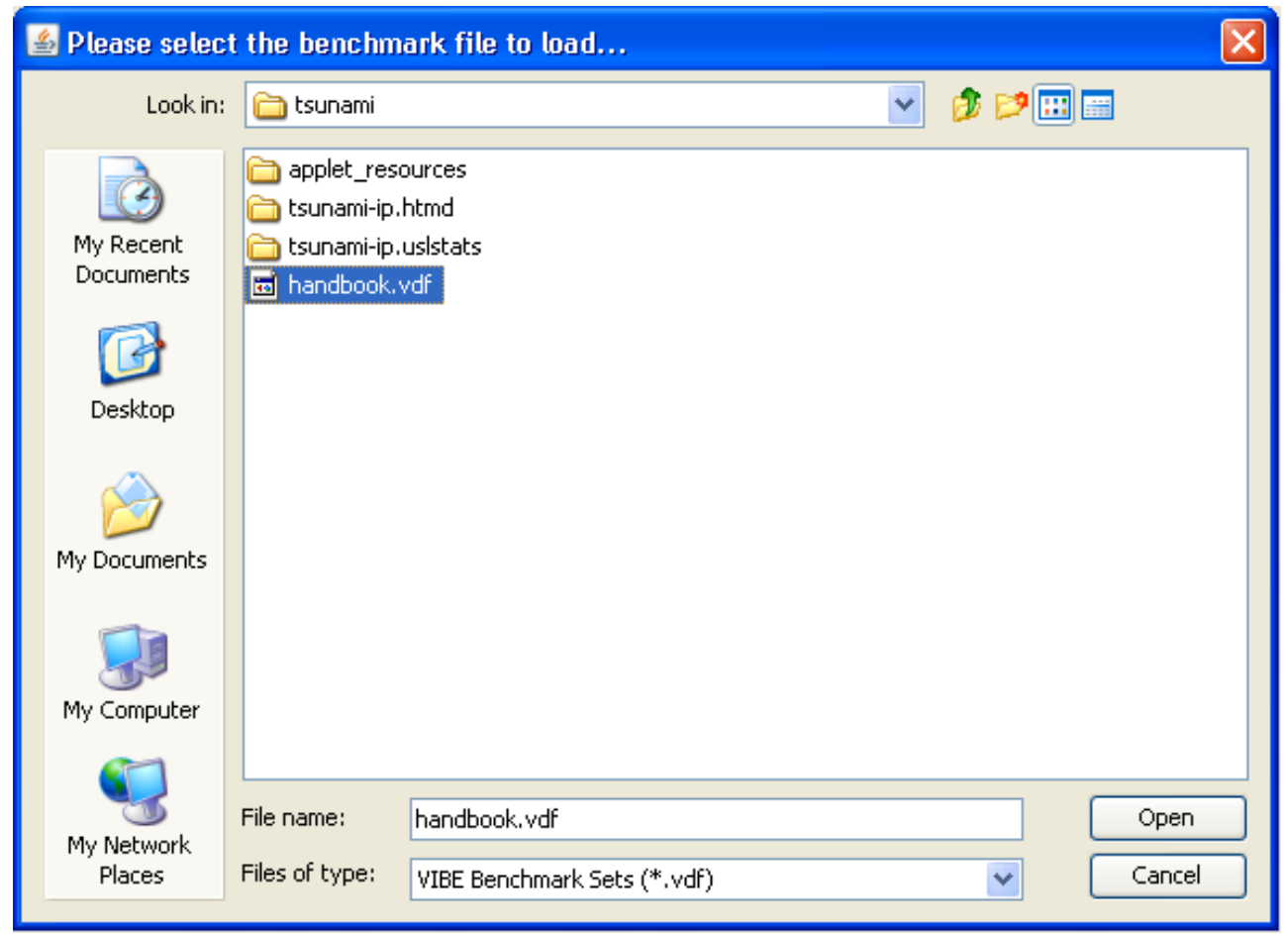

Fig. 160. VIBE file chooser to open the handbook benchmark set.

When the benchmark set loads in VIBE, perform a DICE query to list the benchmark $k_{\text {eff }}$ and benchmark $k_{\text {eff }}$ uncertainty, as shown in Fig. 161.

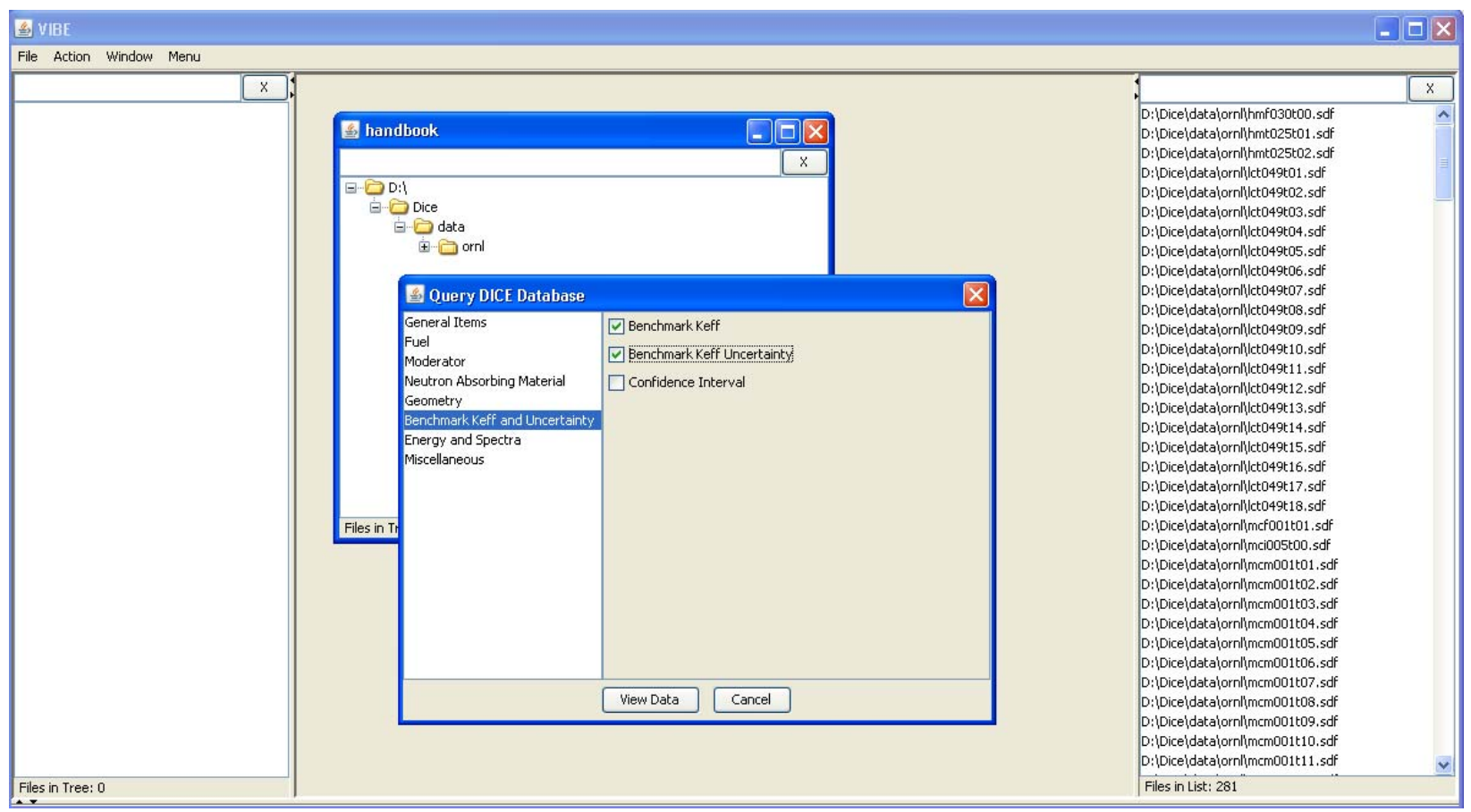

Fig. 161. VIBE DICE database query for benchmark $\boldsymbol{k}_{\text {eff }}$ and $\boldsymbol{k}_{\text {eff }}$ uncertainty. 


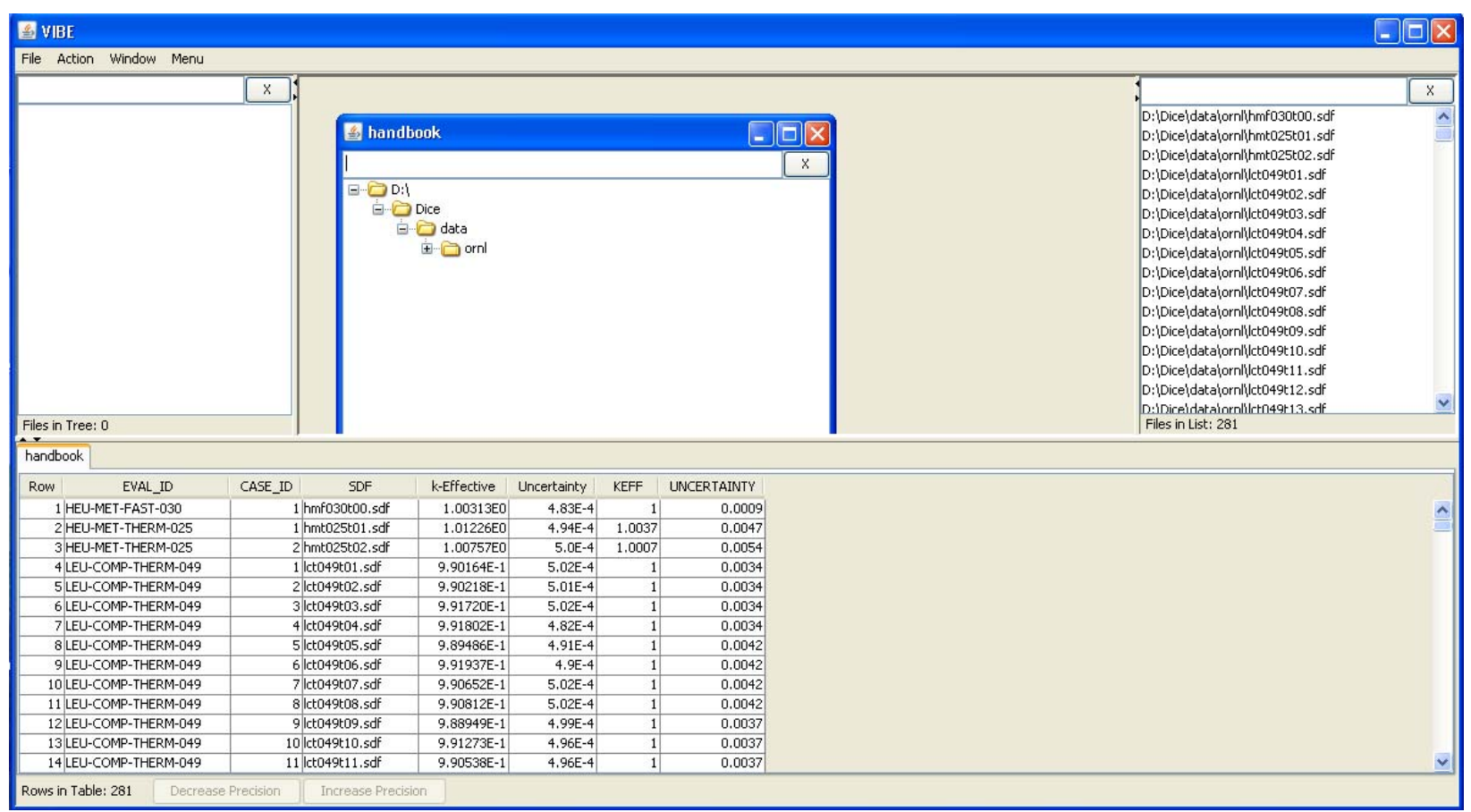

Fig. 162. VIBE query results for benchmark $\boldsymbol{k}_{\text {eff }}$ and $\boldsymbol{k}_{\text {eff }}$ uncertainty.

Although no mechanism currently exists to directly incorporate these results into the USLSTATS input, with a bit of data manipulation, the desired results can be achieved. From the VIBE File menu, select Export Table..., as shown in Fig. 163. In the export dialog, shown in Fig. 164, select . txt to save the table as a text file. Note that a table export will also save group collapsed sensitivity data in the SDF format. After clicking on . $t x t$, save the file in the $\mathrm{C}: \backslash$ tsunami directory as handbook. $t x t$, as shown in Fig. 165.

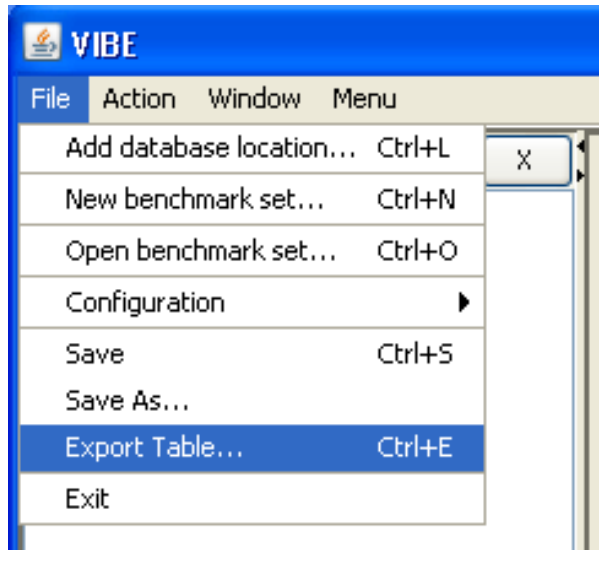

Fig. 163. VIBE file menu item to export data in a table. 


\section{Export Table \\ ? How do you want to export the current table?

$$
\text { As.txt As .sdf Cancel }
$$

Fig. 164. VIBE export dialog.

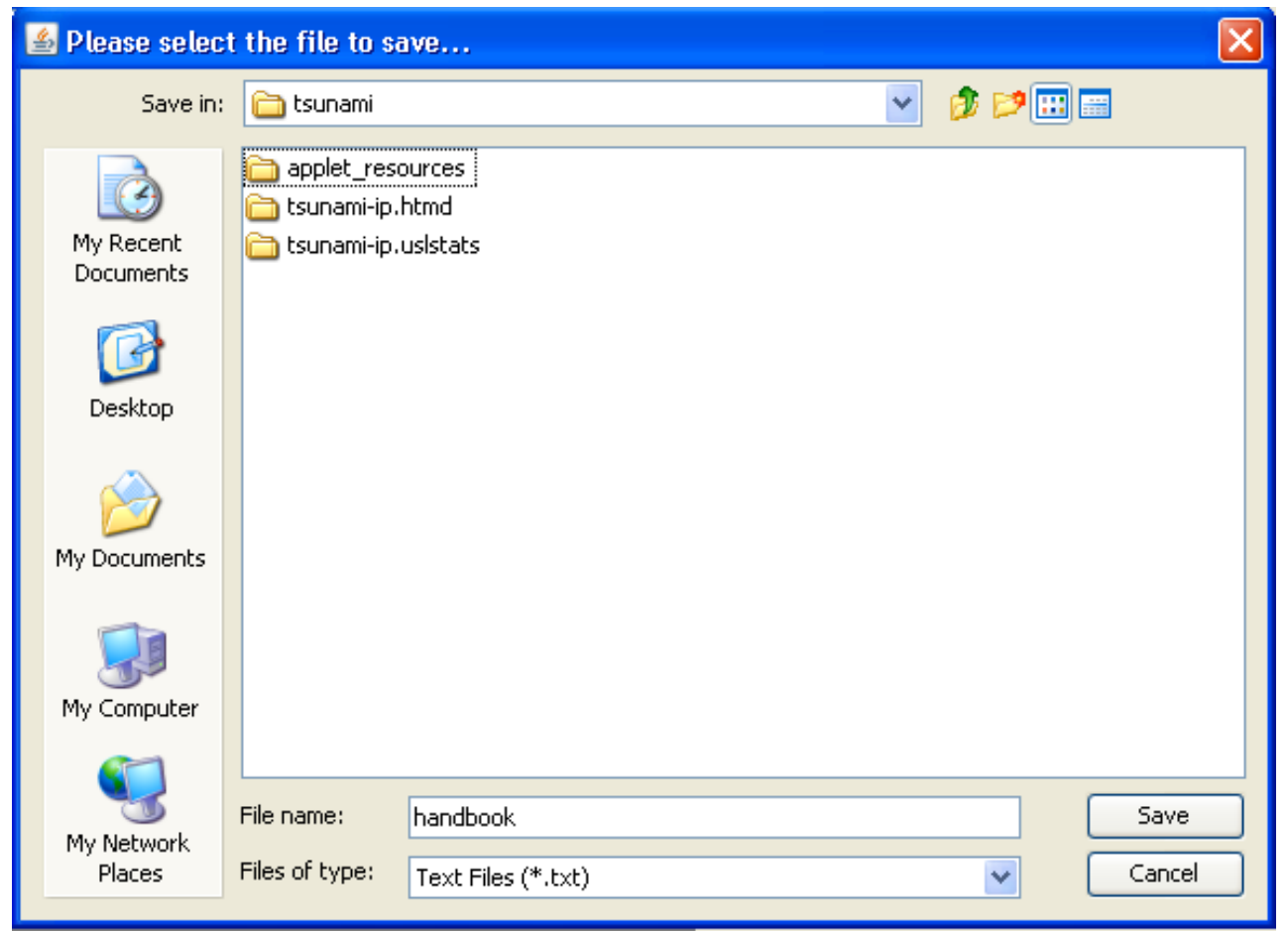

Fig. 165. VIBE file chooser to export table data.

After some manipulations using Excel, the data in the table can be converted into the TSUNAMI-IP input format, where the benchmark $k_{\text {eff }}$ values are designated with $e v=$ for experimental value, and the benchmark $k_{\text {eff }}$ uncertainties are designated with $\mathrm{uv}=$ for uncertainty value. The spreadsheet used to prepare this input is provided with SCALE as C: \scale6 $\backslash p$ pimers $\backslash T S U N A M I \backslash f i l e s \backslash h a n d b o o k-d a t a . x l s$. Future plans call for tighter integration of VIBE and ExSITE so the use of Excel to manipulate the data will eventually not be required. ExSITE with the updated TSUNAMI-IP input is shown in Fig. 166. 


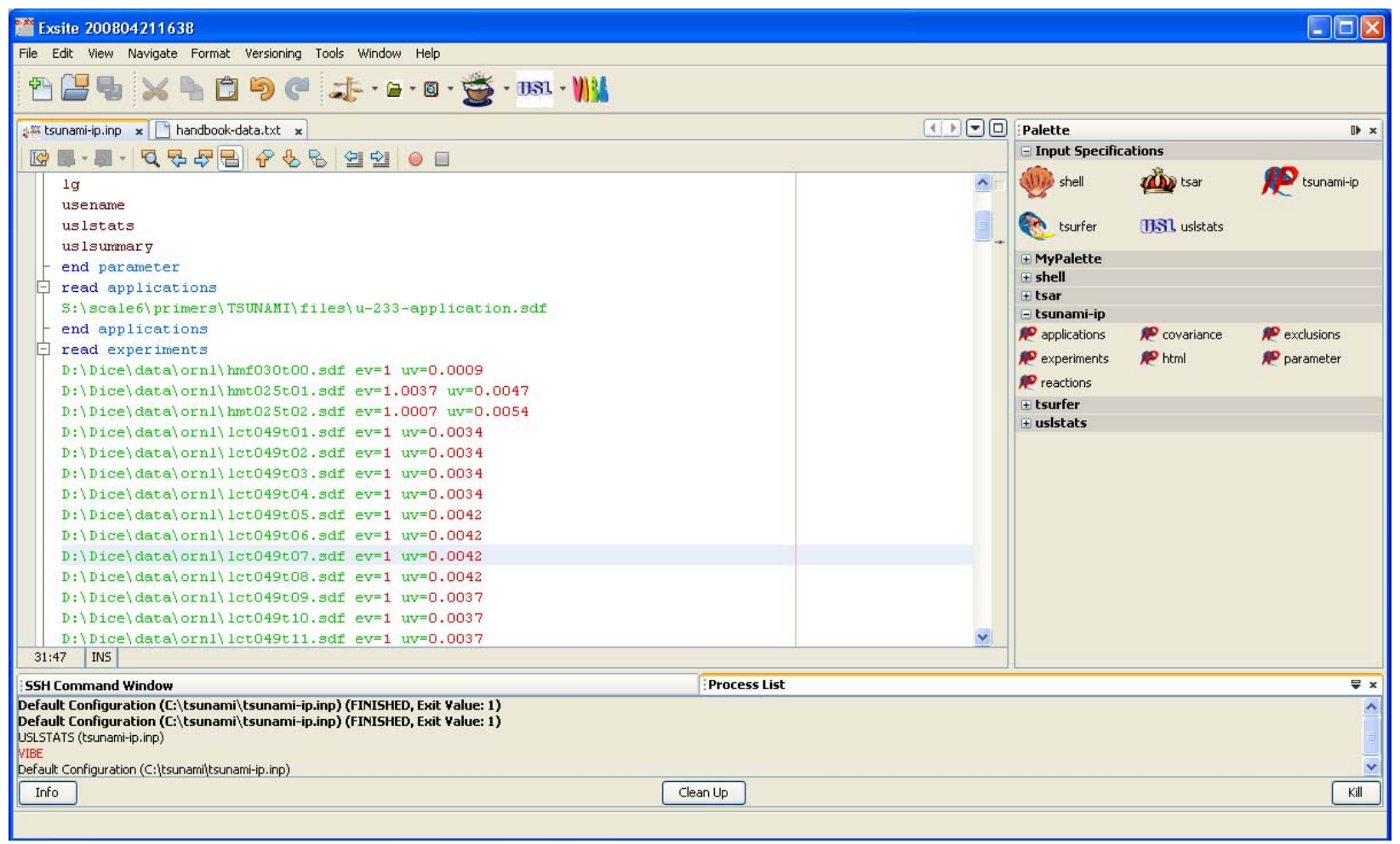

Fig. 166. ExSITE with TSUNAMI-IP input including benchmark $\boldsymbol{k}_{\text {eff }}$ and benchmark $\boldsymbol{k}_{\text {eff }}$ uncertainty values.

After executing the TSUNAMI-IP calculation and opening USLSTATS with the updated input files, the updated USLSTATS data appear as shown in Fig. 171. In the read data section of the input, the values on each row represent an experiment with its $c_{k}$ value, the ratio of the calculated-to-measured $k_{\text {eff }}$ values, and the relative experimental uncertainty. Note that the values shown in Fig. 167 differ from those shown in Fig. 150 and more accurately represent the experimental data. 


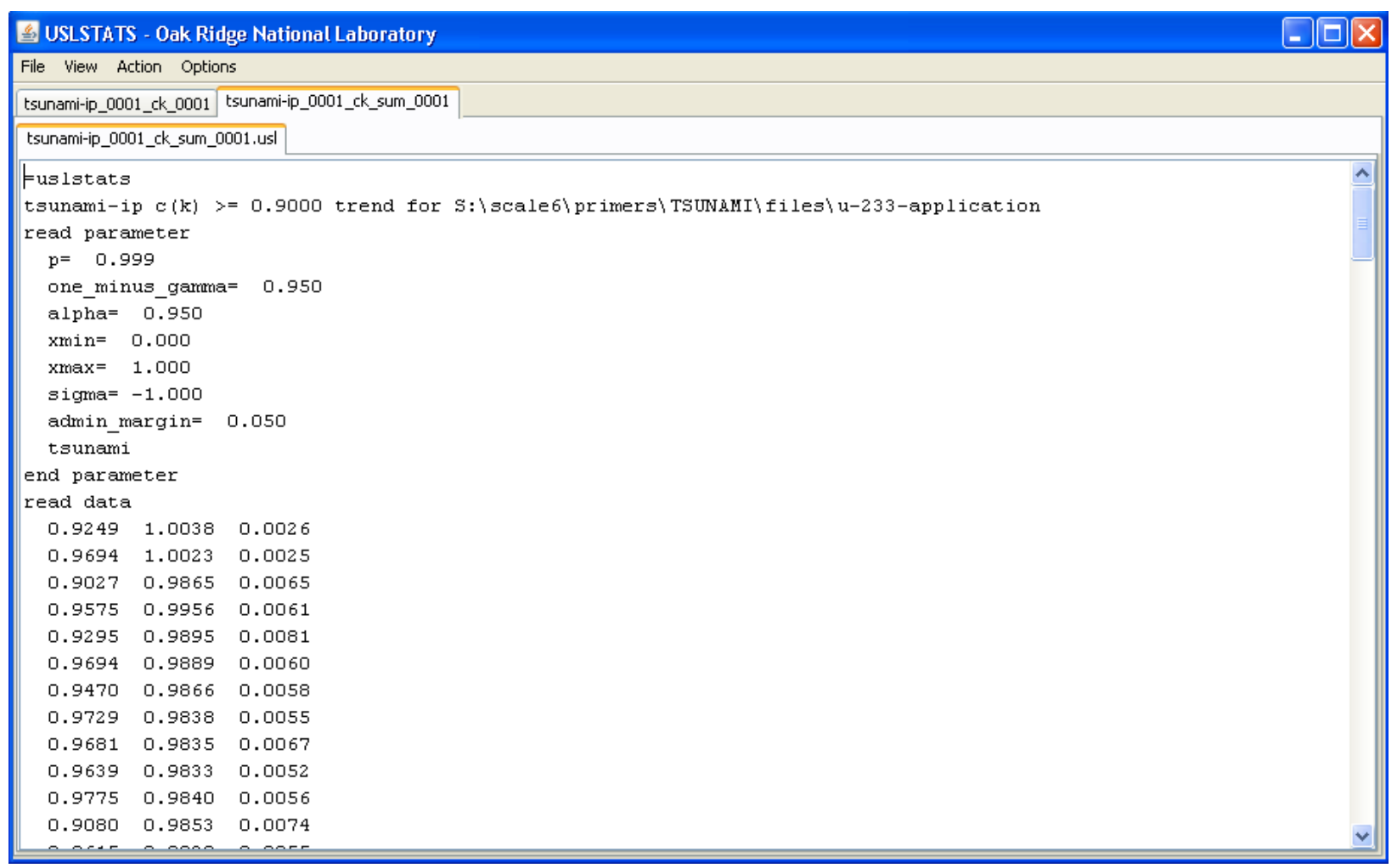

Fig. 167. ULSTATS input using benchmark $\boldsymbol{k}_{\text {eff }}$ and benchmark $\boldsymbol{k}_{\text {eff }}$ uncertainty values.

Repeating the USLSTATS analysis provides similar results to those from the previous analysis, with the trend plot shown in Fig. 168. Here many of the benchmark $k_{\text {eff }}$ uncertainty values have increased relative to the initial assumption of $0.3 \% \Delta \mathrm{k} / \mathrm{k}$. The bias decreased slightly to $1.00487 \% \Delta \mathrm{k} / \mathrm{k}$, and the bias uncertainty increased to $0.019365 \% \Delta \mathrm{k} / \mathrm{k}$. 


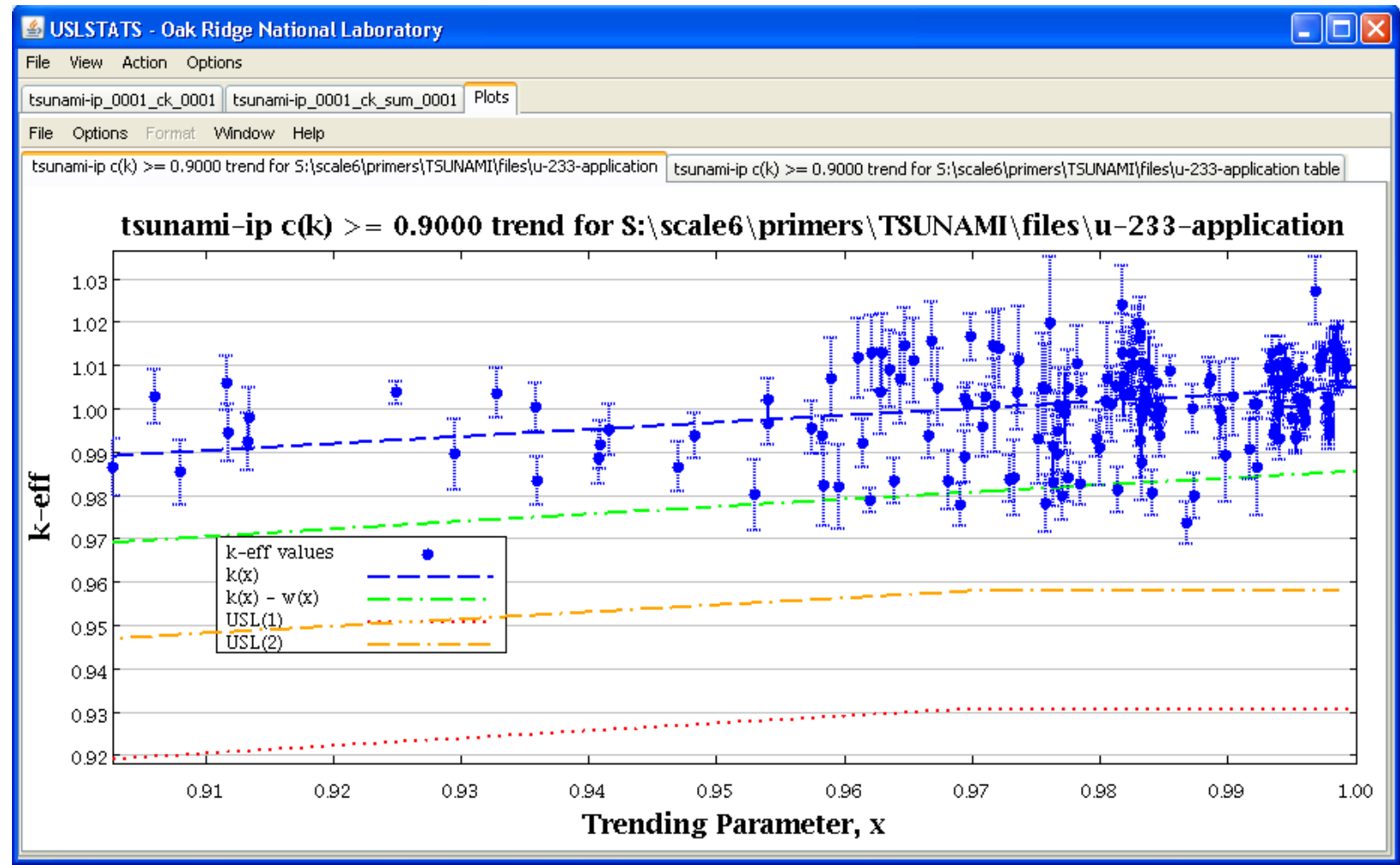

Fig. 168. USLSTATS trend plot including benchmark $\boldsymbol{k}_{\text {eff }}$ and benchmark $\boldsymbol{k}_{\text {eff }}$ uncertainties for experiments with $c_{k} \geq 0.9$ for ${ }^{233} \mathrm{U}$ application.

\subsection{GAP ANALYSIS}

Because the bias and bias uncertainty analysis procedure detailed in the previous section includes extrapolation from experiments to the application in terms of uncertainty, there will always be a gap that must be spanned. The statistical treatment of USLSTATS accounts for extrapolations in terms of known quantities, such as small concentrations or spectral variations for applications that fundamentally contain the same materials as many experiments. However, there may be a gap in the validation for certain materials that are present in the application but not present in available experiments. In some cases a material is tested in a benchmark, but the experiment is insufficiently sensitive to the desired material, either spectrally or simply in terms of magnitude. If the material is absent from the trending analysis, the statistical analysis cannot adequately predict what impact the material might have on the computational bias. However, using the uncertainty data for the application, the magnitude of the bias, although not the sign, can be bounded.

The TSUNAMI tools present a gap analysis methodology for quantifying an additional margin to subcriticality due to uncertainties in the safety application that exceed the area of applicability of the critical experiment suite selected for validation. Where the material is completely absent from the experiments, the uncertainty of the application due to the given material can be extracted from the TSUNAMI-IP extended uncertainty edit, shown in Fig. 124. Where the material is present in the experiments but provided inadequate coverage, the TSUNAMI-IP penalty calculation determines the uncertainty remaining after the coverage for each groupwise, nuclide-reaction-specific sensitivity from the suite of benchmark experiments and deducts it from the sensitivity of the safety application. The penalty procedure is described in detail in the SCALE manual section for TSUNAMI-IP. 
To request the penalty assessment for the application, enter penalty in the TSUNAMI-IP parameter data. To view an extended edit of the penalty contribution from each covariance matrix, enter penlong in the TSUNAMI-IP parameter data, as shown in Fig. 169.

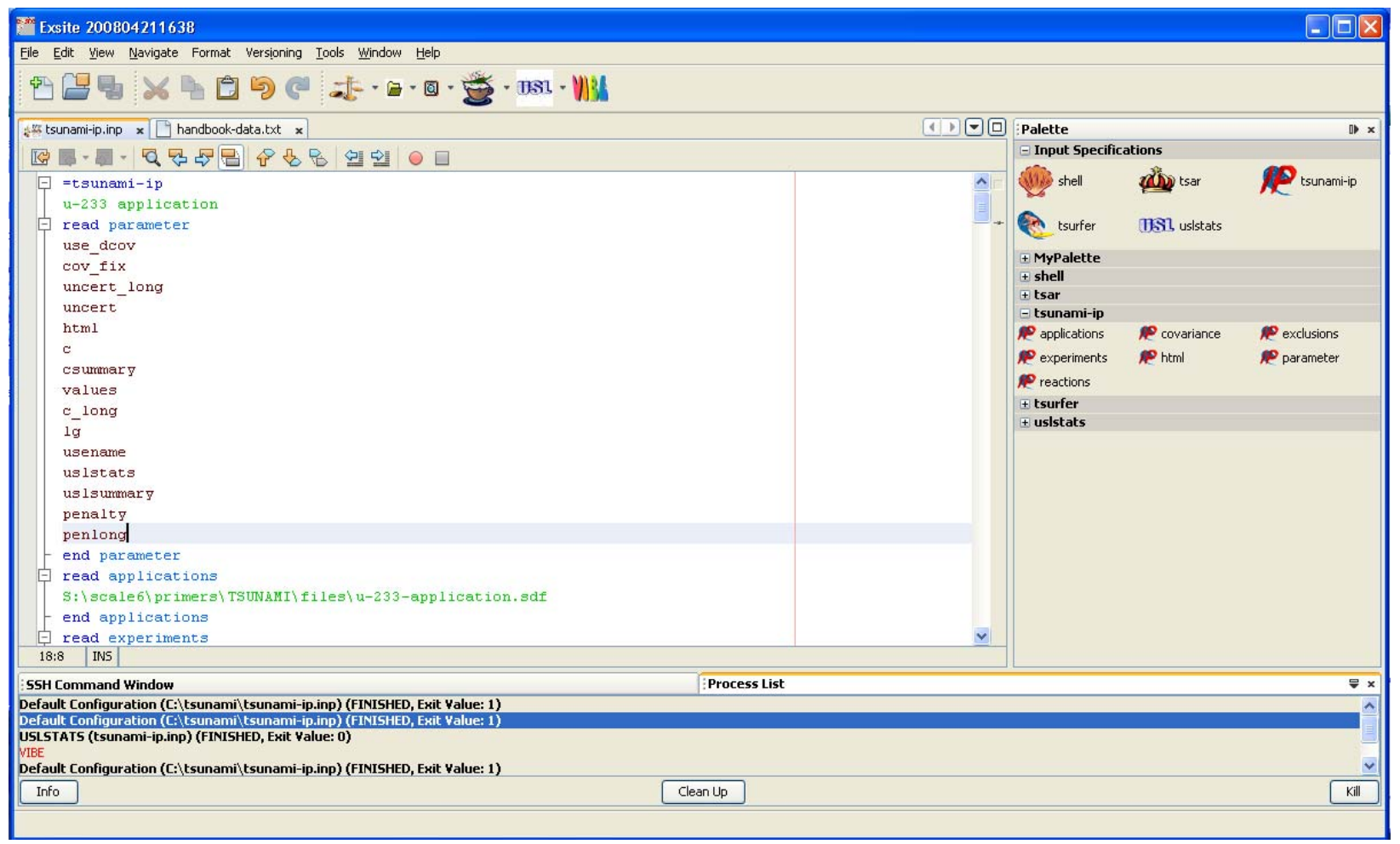

Fig. 169. ExSITE with TSUNAMI-IP input updated to perform penalty calculations.

After running the updated TSUNAMI-IP input and refreshing the HTML output, the Penalty and Uncertainty Assessment submenu will contain entries for Penalty Summary, shown in Fig. 170, and Extended Penalty Assessment, shown in Fig. 171. As shown in Fig. 124, the original uncertainty of the application due to uncertainties in the cross section is $1.067 \% \Delta \mathrm{k} / \mathrm{k}$. After the coverage provided by the 281 experiments from the handbook is applied, the remaining uncertainty, as shown in Fig. 170, is $0.0164 \% \Delta \mathrm{k} / \mathrm{k}$. Thus, approximately $99 \%$ of the potential sources of computational bias are accounted for in the validation suite available for the ${ }^{233} \mathrm{U}$ application. The remaining uncovered or undercovered data are revealed in the extended penalty assessment of Fig. 171. Here the remaining uncertainties are due to components of the stainless steel tank. However, in other applications, exotic structural components or poisons, such as fission products, may not have adequate validation coverage, and the TSUNAMI gap analysis techniques can provide justification for establishing additional margin to subcriticality. 


\begin{tabular}{|l|l|l|l|l|l|}
\hline G TSUNAMI-IP- Windows Internet Explorer & \\
\hline General \\
Information \\
Messages
\end{tabular}

Fig. 170. TSUNAMI-IP penalty summary edit in HTML output.

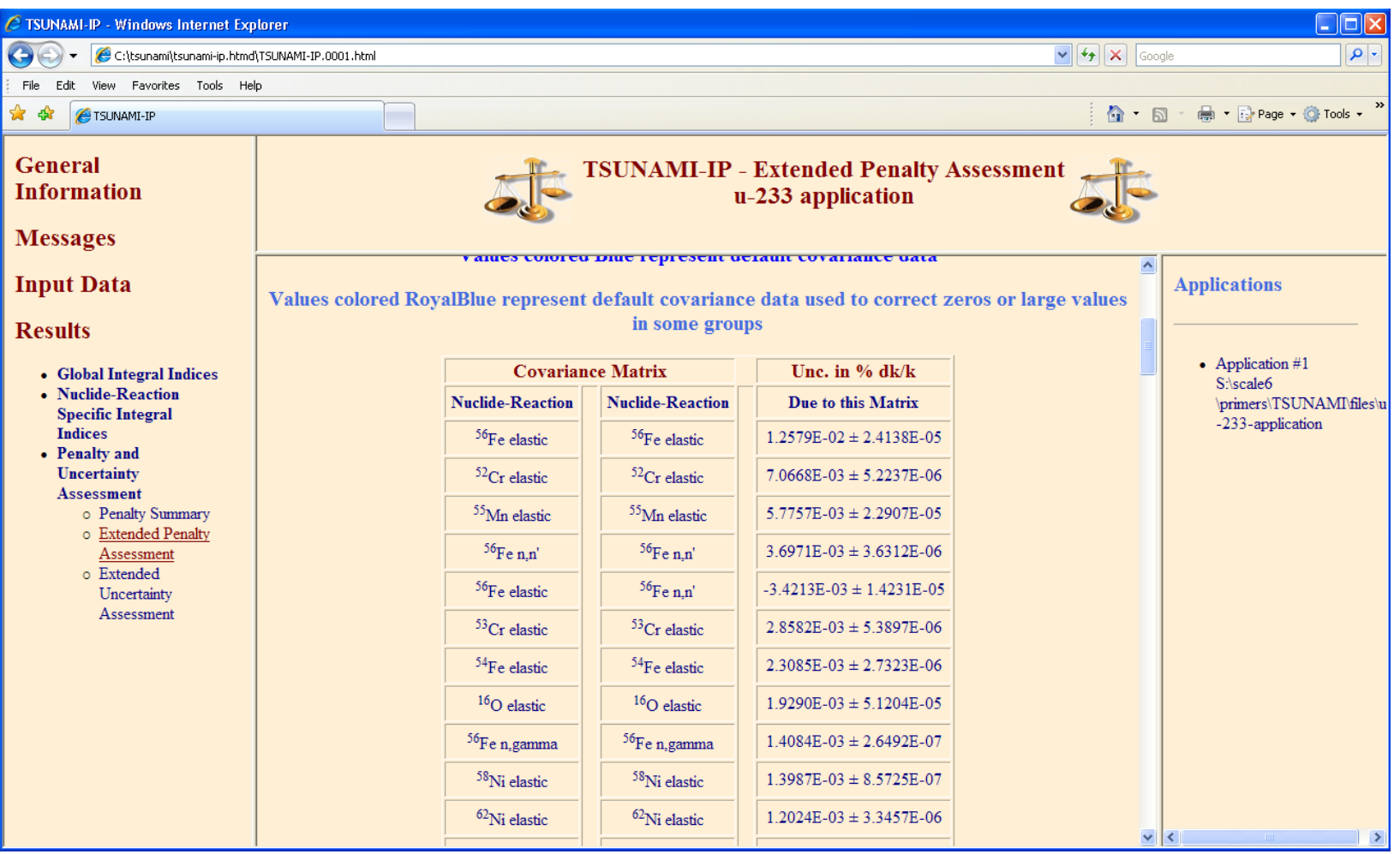

Fig. 171. TSUNAMI-IP extended penalty assessment edit in HTML output. 
The coverage provided by the benchmark set can be visualized with the composite sensitivity data feature of TSUNAMI-IP. When prtcomp is entered in the TSUNAMI-IP input, as shown in the palette dialog in Fig. 172, a new SDF will be generated with a composite of the best-matching sensitivity data from all experiments for each nuclide-reaction pair specified in the reaction data block of TSUNAMI-IP.

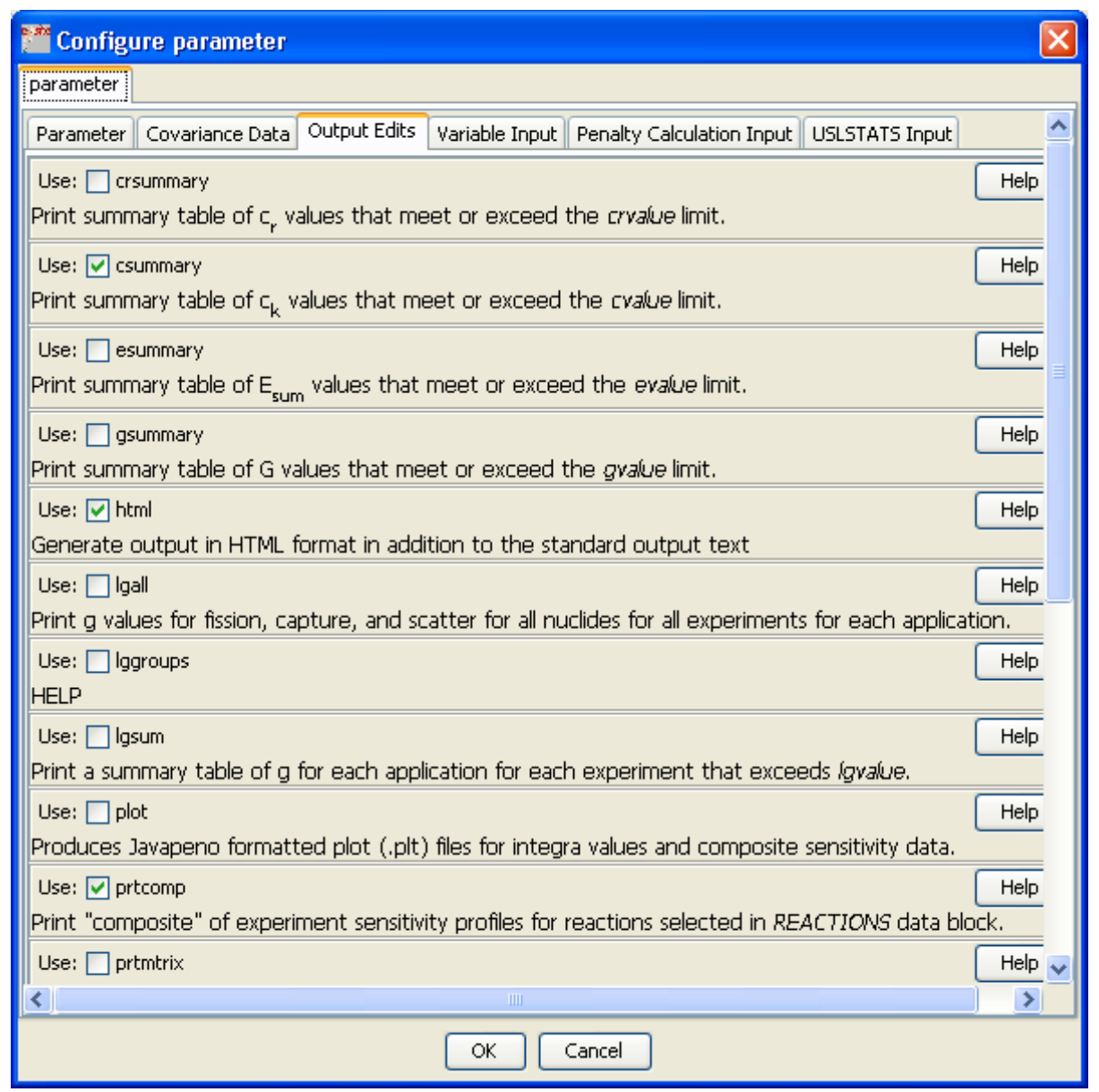

Fig. 172. ExSITE palette dialog for TSUNAMI-IP output edit parameters.

To add a reactions input block using ExSITE, open the main palette dialog, and check the box next to reactions. A new tab will appear at the top of the palette dialog, as shown in Fig. 173. To examine composite coverage for the top three reactions in the extended penalty edit, enter the appropriate data in the reactions block using either the palette dialog or manual entries, as shown in Fig. 174. 


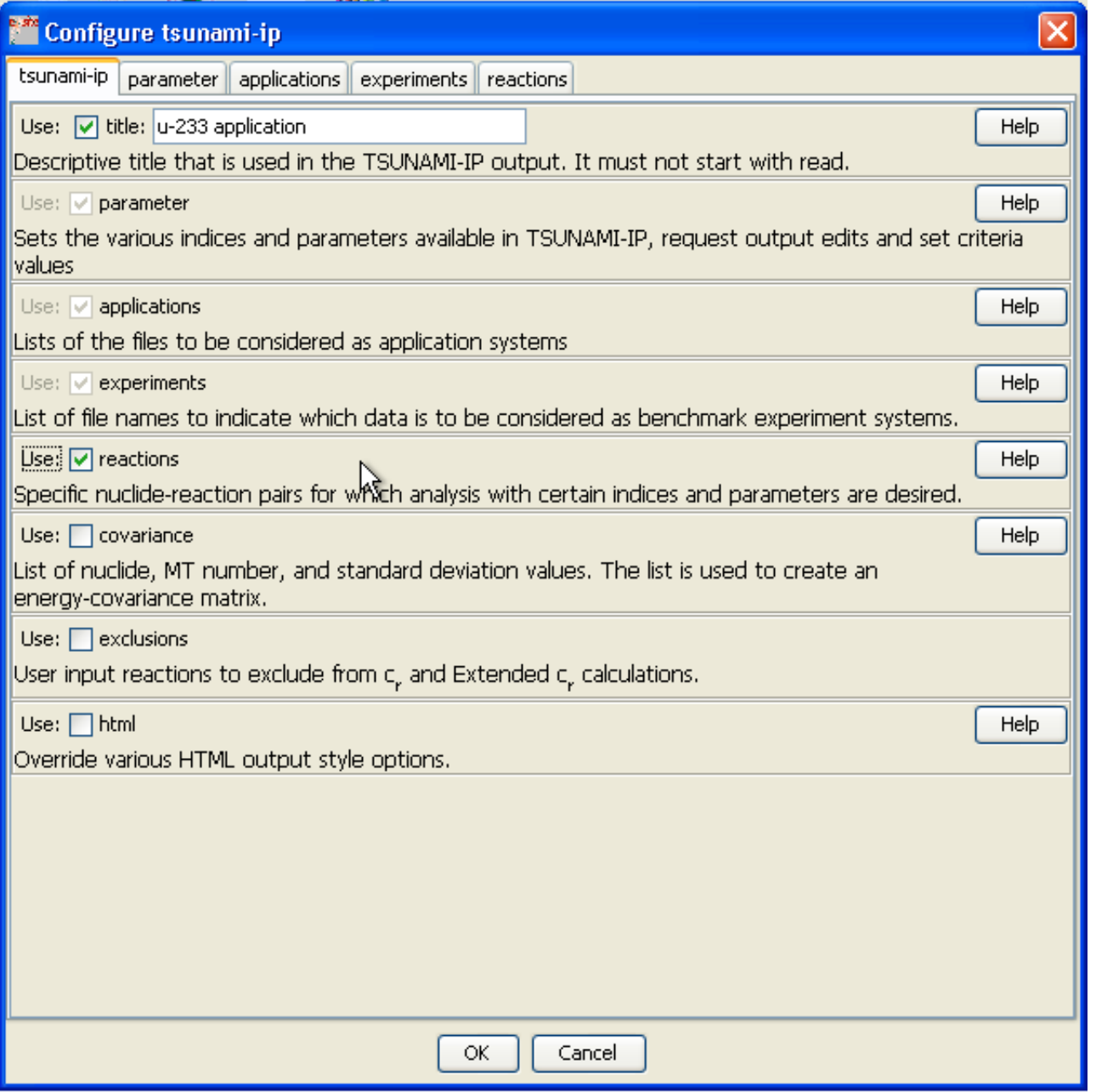

Fig. 173. ExSITE palette dialog to add reactions block to TSUNAMI-IP input. 


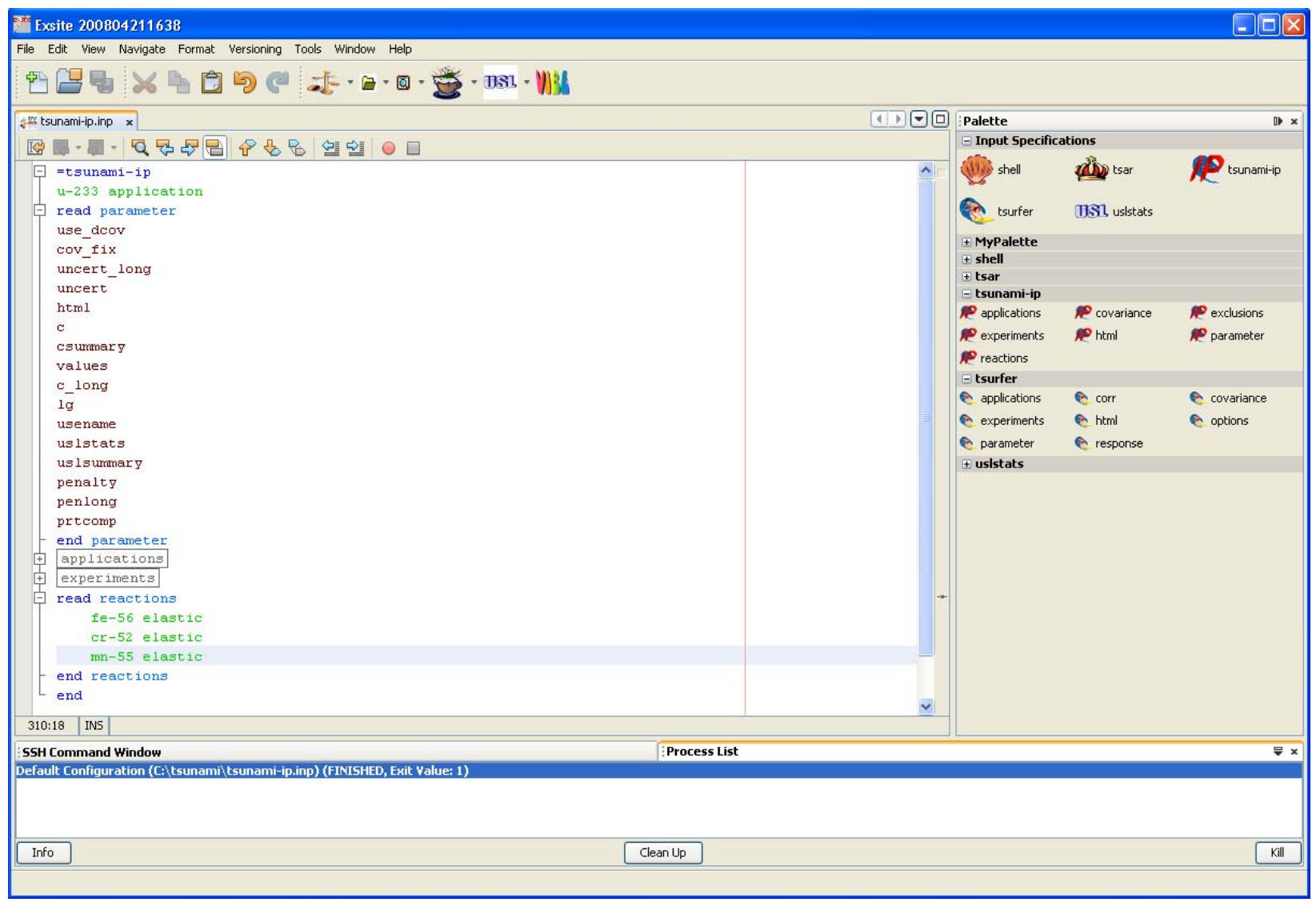

Fig. 174. ExSITE with TSUNAMI-IP input updated to add reactions data.

After running the calculation and refreshing the TSUNAMI-IP output, a new submenu for Coverage and Completeness Assessment will be available. Opening the Composite Sensitivity Data Plot edit will load the composite SDF in Javapeño. For each nuclide-reaction pair requested in the reactions block, three profiles are available for plotting. The Application data represent the sensitivity of the application itself. The Composite data represent the sensitivity for each group from any experiment considered in the calculation that best matches the sensitivity of the application, truncated so that they cannot exceed the sensitivity value of the application itself. The Cut Composite is the same as the composite, except that only experiments with $c_{k}$ values exceeding cvalue are included. Experiments with lower $c_{k}$ values are "cut" from the calculation. The cut composite sensitivity profiles are the ones used in the calculation of the penalty.

As shown in Fig. 175, the application sensitivity for ${ }^{56} \mathrm{Fe}$ elastic scattering, shown in green, slightly exceeds the composite data, shown in red, in most energy groups. However, the application sensitivity is approximately twice the magnitude of the cut composite profile, shown in blue, for most energy groups. From this plot, it appears that experiments with lower $c_{k}$ values are more sensitive to ${ }^{56} \mathrm{Fe}$ elastic scattering that those with higher $c_{k}$ values. Although these sensitivities are available and therefore the cross sections are validated with at least one experiment, it is not clear how to assess the bias due to this material through trending analysis because the other materials in the experiments are dissimilar from those in the application. 


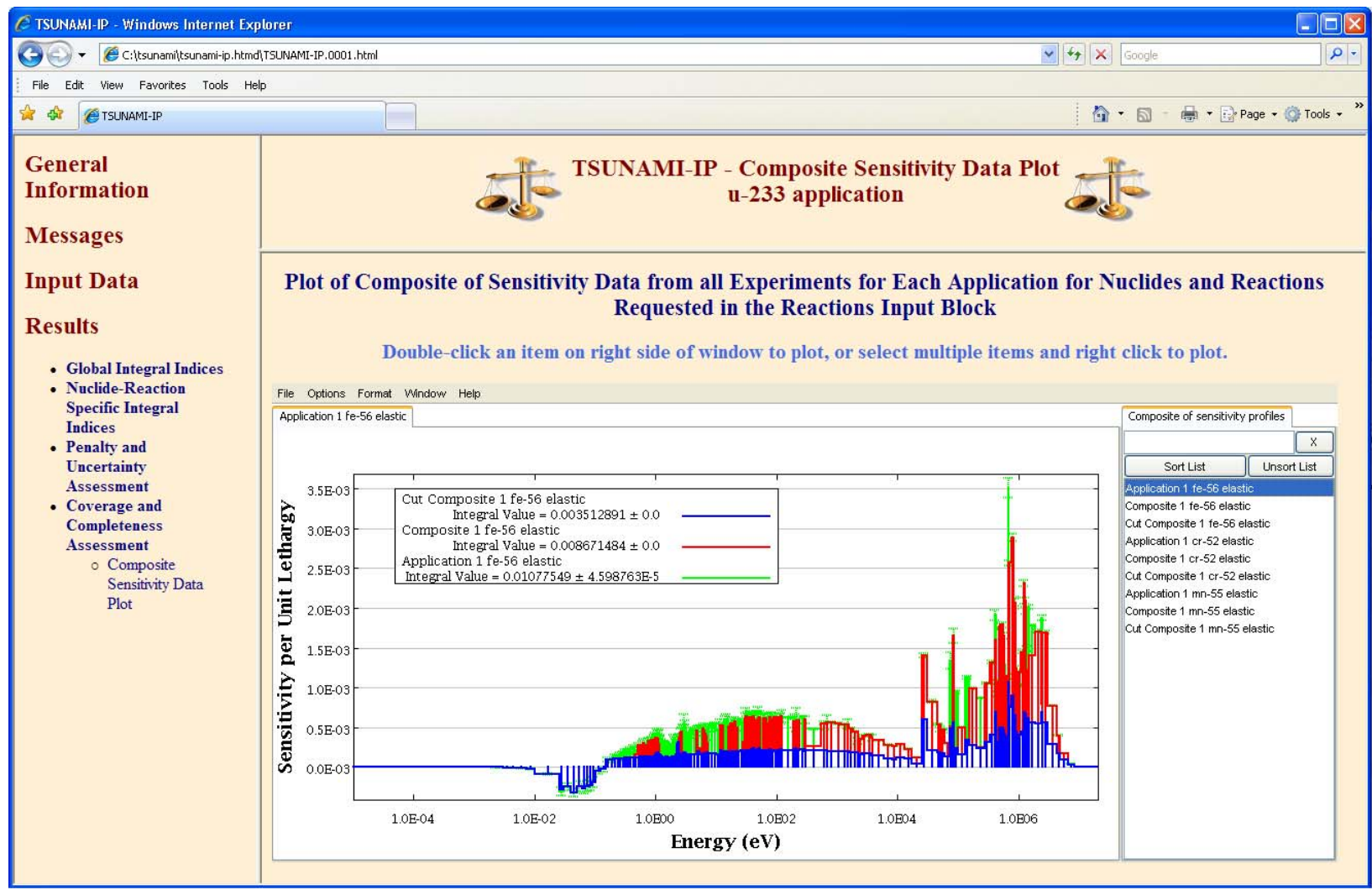

Fig. 175. TSUNAMI-IP composite sensitivity data plot in HTML output. 


\section{BIAS ASSESSMENT WITH TSURFER}

A new capability for SCALE 6 allows the prediction of computational biases with the nuclear data adjustment tool TSURFER, which is based on a generalized linear least squares approach. TSURFER identifies a single set of adjustments to nuclear data that will result in the computational models all producing $k_{\text {eff }}$ values close to their experimental $k_{\text {eff }}$ value. Then the same data adjustments are used to predict an unbiased $k_{\text {eff }}$ value for the application and an uncertainty on the adjusted $k_{\text {eff }}$ value. The difference between the originally calculated $k_{\text {eff }}$ value and the new postadjustment $k_{\text {eff }}$ value represents the bias in the original calculation, and the uncertainty in the adjusted value represents the uncertainty in this bias. If similar experiments are available to validate the use of a particular nuclide in the application, the uncertainty of the bias for this nuclide is reduced. In TSURFER, experiments that are dissimilar from the application can still provide useful information for bias assessment if at least one material demonstrates similar sensitivities to those of the applications. If similar experiments are not available to validate a particular nuclide, the uncertainty in the bias for the given nuclide is high. Thus, with a complete set of experiments to validate important components in the application, a precise bias with a small uncertainty can be predicted. Where the experimental coverage is lacking, a bias can be predicted with an appropriately large uncertainty. As users gain experience with TSURFER, it is expected to become a preferred tool for rigorous bias and bias uncertainty determination, particularly for applications for which nearly identical critical experiments are not available.

The input for TSUNAMI-IP and TSURFER are similar in many ways. Both codes require SDFs to identify the applications and experiments, and both codes allow corrections to be made to the crosssection-covariance data. Because of the similarities of the input file, it is convenient to save the existing tsunami-ip.inp file as tsurfer.inp using Save As... from the ExSITE File menu, then change the sequence specification record at the top of the input from =tsunami-ip to =tsurfer, as shown in Fig. 176. Note that ExSITE underlines several parameters, identifying them as errors in the TSURFER input. These parameters from TSUNAMI-IP are not valid in TSURFER and must be deleted. However, the parameters related to covariance data remain valid and should be left in the input for use in the TSURFER analysis.

The experiment, $e v=$, and uncertainty, $u v=$, values that were optional input to TSUNAMI-IP are required in TSURFER. 


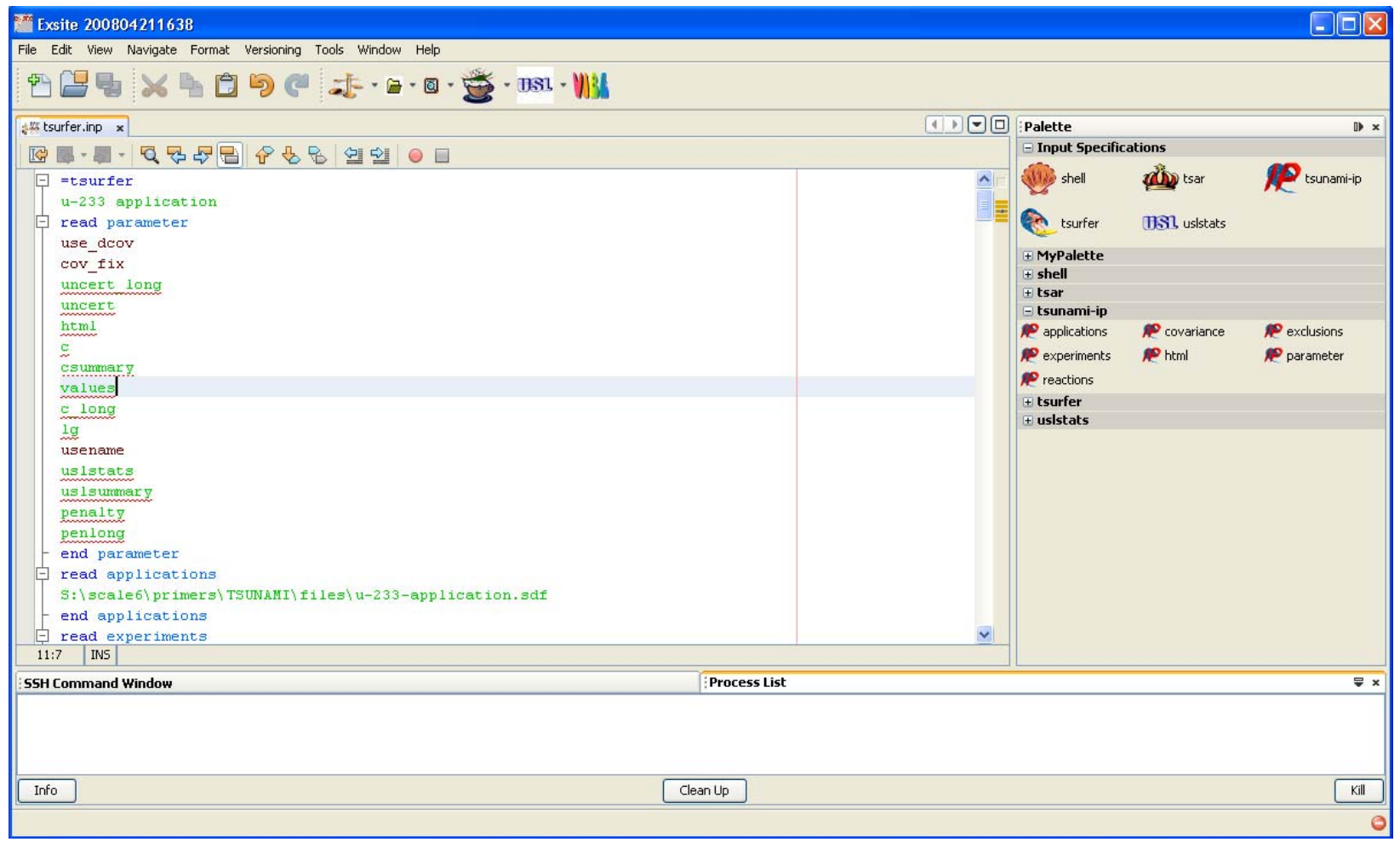

Fig. 176. Conversion of TSUNAMI-IP input to TSURFER input using ExSITE.

Once the invalid input parameters are deleted, the palette dialogs for TSURFER can be used to input additional input parameters. In the Calculation Options parameter tab, shown in Fig. 177, the ref_app is defined as 282nd SDF in the input file, the ${ }^{233} \mathrm{U}$ application. The reference application is used for additional output edits regarding sources of bias. Many applications can be input to TSURFER, but only one can be defined as the reference application. In the Output Edits parameter tab, shown in Fig. 178, the option to print cross-section adjustments, print_adjustments, is enabled, and the usename parameter from the TSUNAMI-IP input is preserved. In the Covariance Data tab, shown in Fig. 179, the cov_fix and use_dcov options from TSUNAMI-IP are preserved, and the TSURFER parameter to return the postadjustment cross-section-covariance library to the user's directory, return_adj_cov, is set. The updated TSURFER input is shown in Fig. 180. 


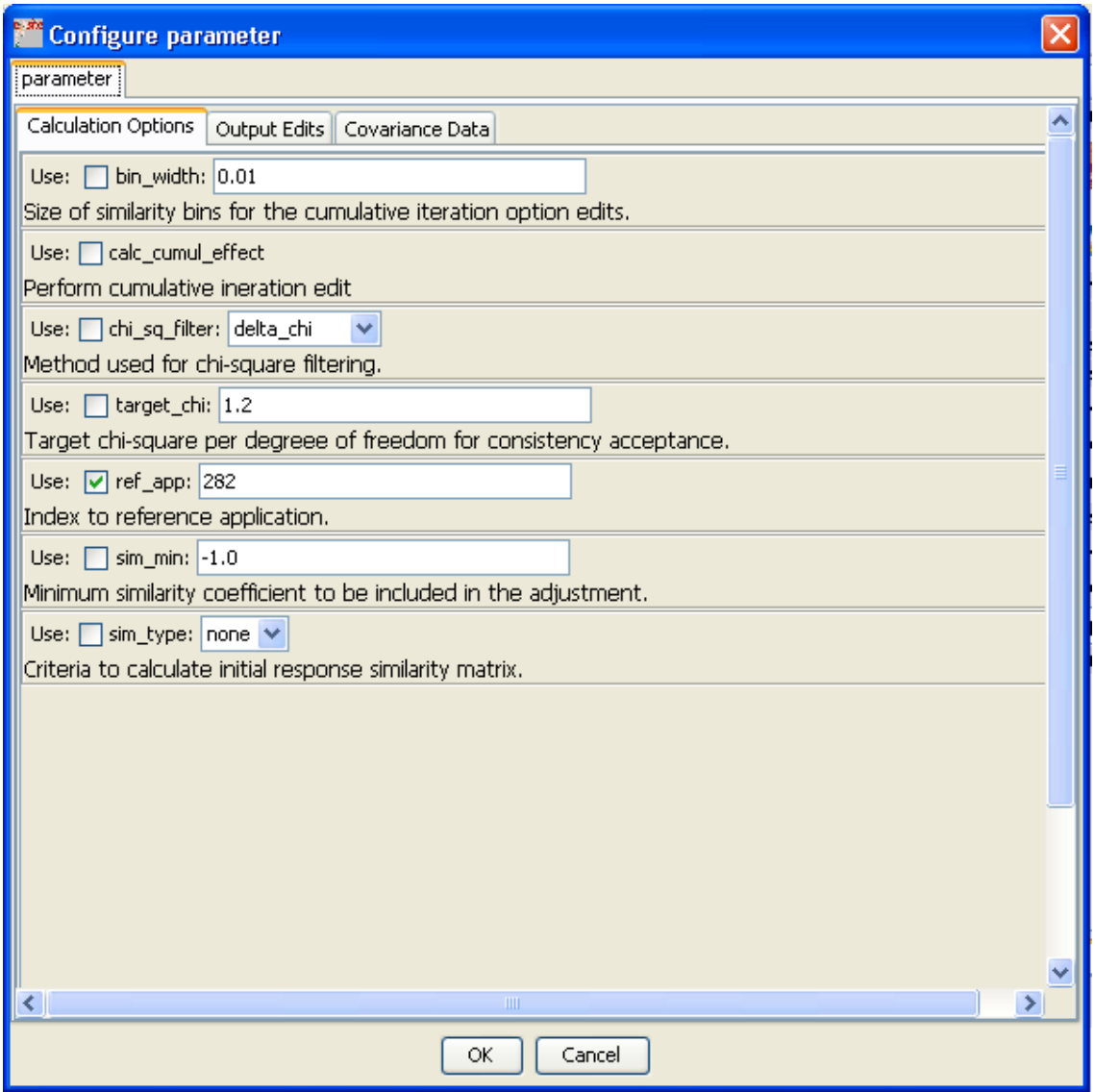

Fig. 177. ExSITE palette dialog for TSURFER calculation options parameters. 


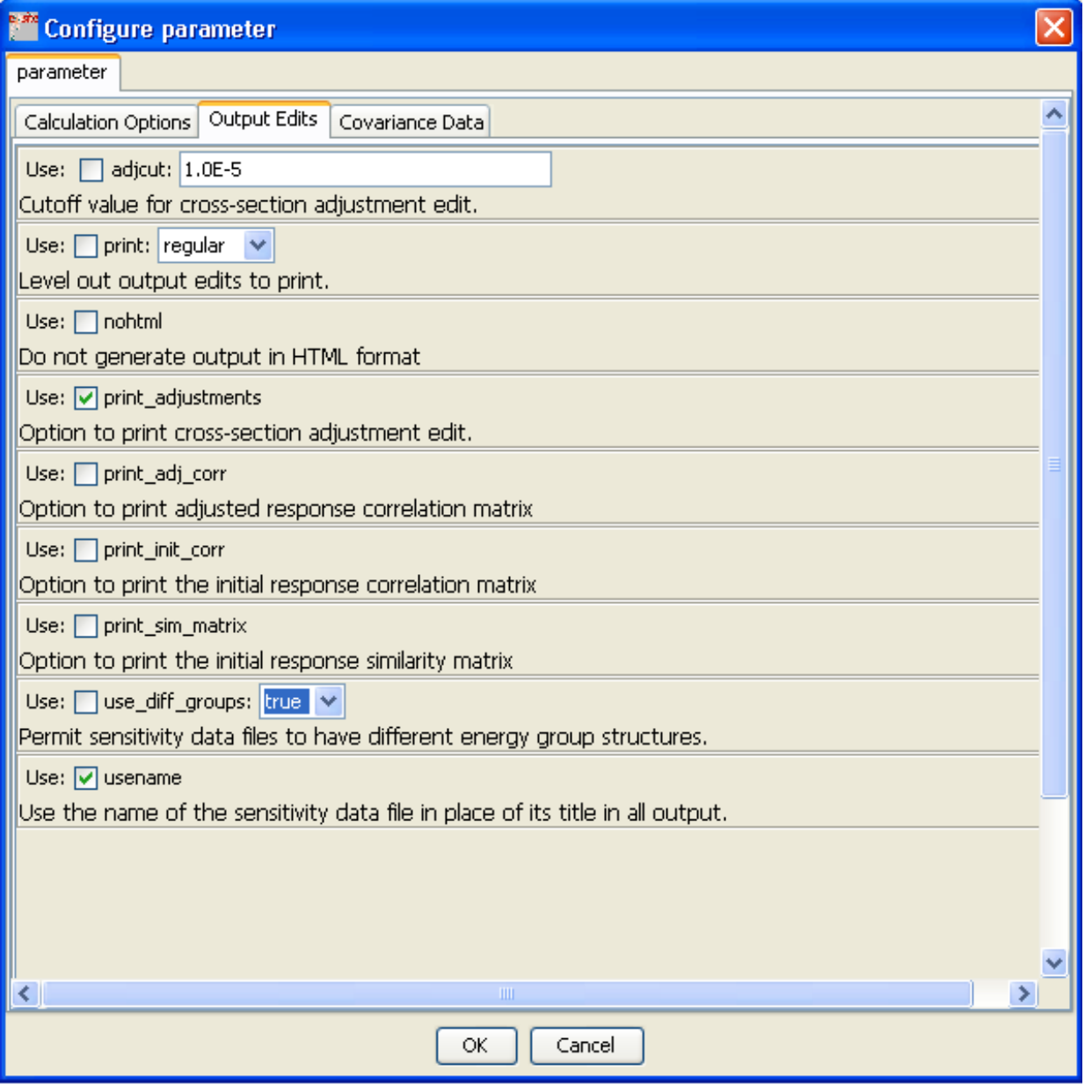

Fig. 178. ExSITE palette dialog for TSURFER output edits parameters. 


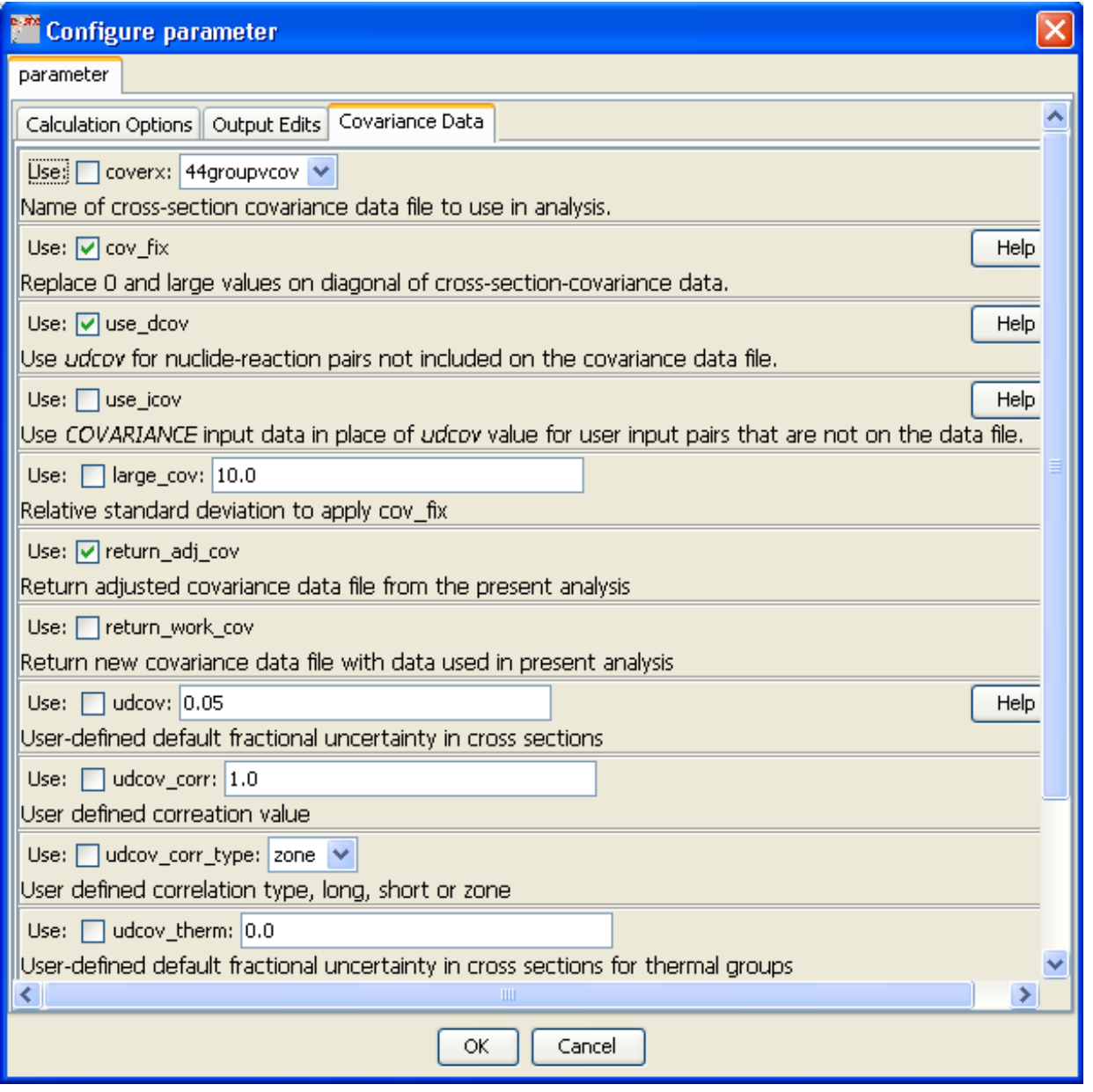

Fig. 179. ExSITE palette dialog for TSURFER covariance data parameters. 


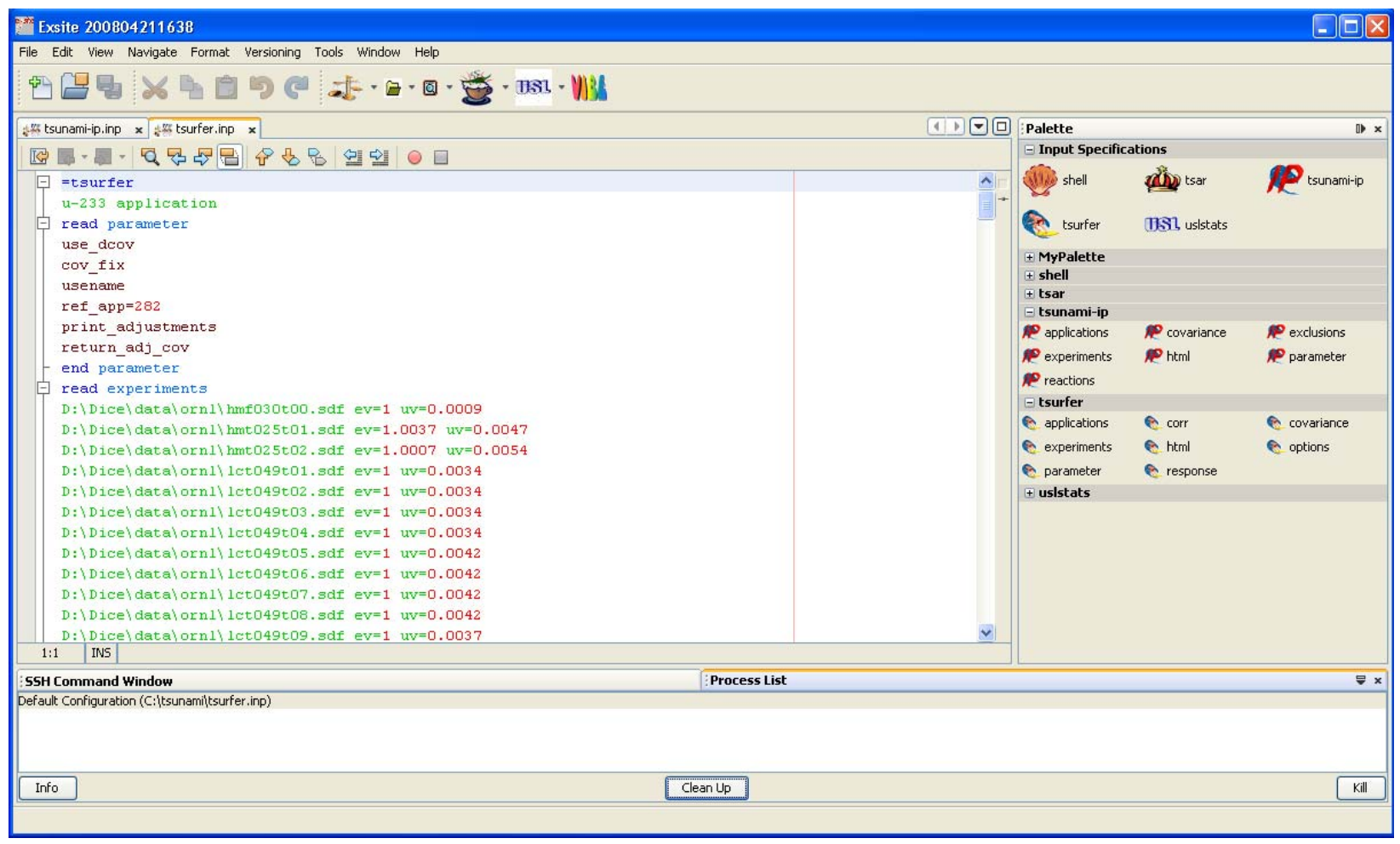

Fig. 180. TSURFER input with updated parameter data in ExSITE.

An additional important input to the TSURFER calculation is the set of correlations between experiment

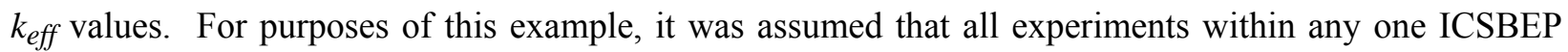
Handbook evaluation were $90 \%$ correlated and that there were no correlations between experiments in different evaluations. This approximation is arbitrary. In reality, the same fuel rods, plates, or solutions are used in multiple evaluations, and the correlations are far more complex than the simple assumption used here. However, this provides an approximate input for TSURFER and will be sufficient for demonstration purposes. There is currently an effort under way through the ICSBEP to quantify correlations between experimental configurations and provide that information through DICE. It is hoped that when these data are available, VIBE will be able access these correlations to provide convenient input for TSURFER. For now, the $90 \%$ correlations are input to the corr block of TSURFER, as shown in Fig. 181. Note that experiments in the cor $r$ block are identified by number. The number corresponds to the order of all SDFs input to TSURFER, whether they are applications or experiments. To simplify the input, the experiments have been moved to before the applications, so the correlation values will correspond to the experiment number. A spreadsheet used to prepare the corr input is provided with SCALE as C: \scale6\primers \TSUNAMI \files \handbook-data .xls. 


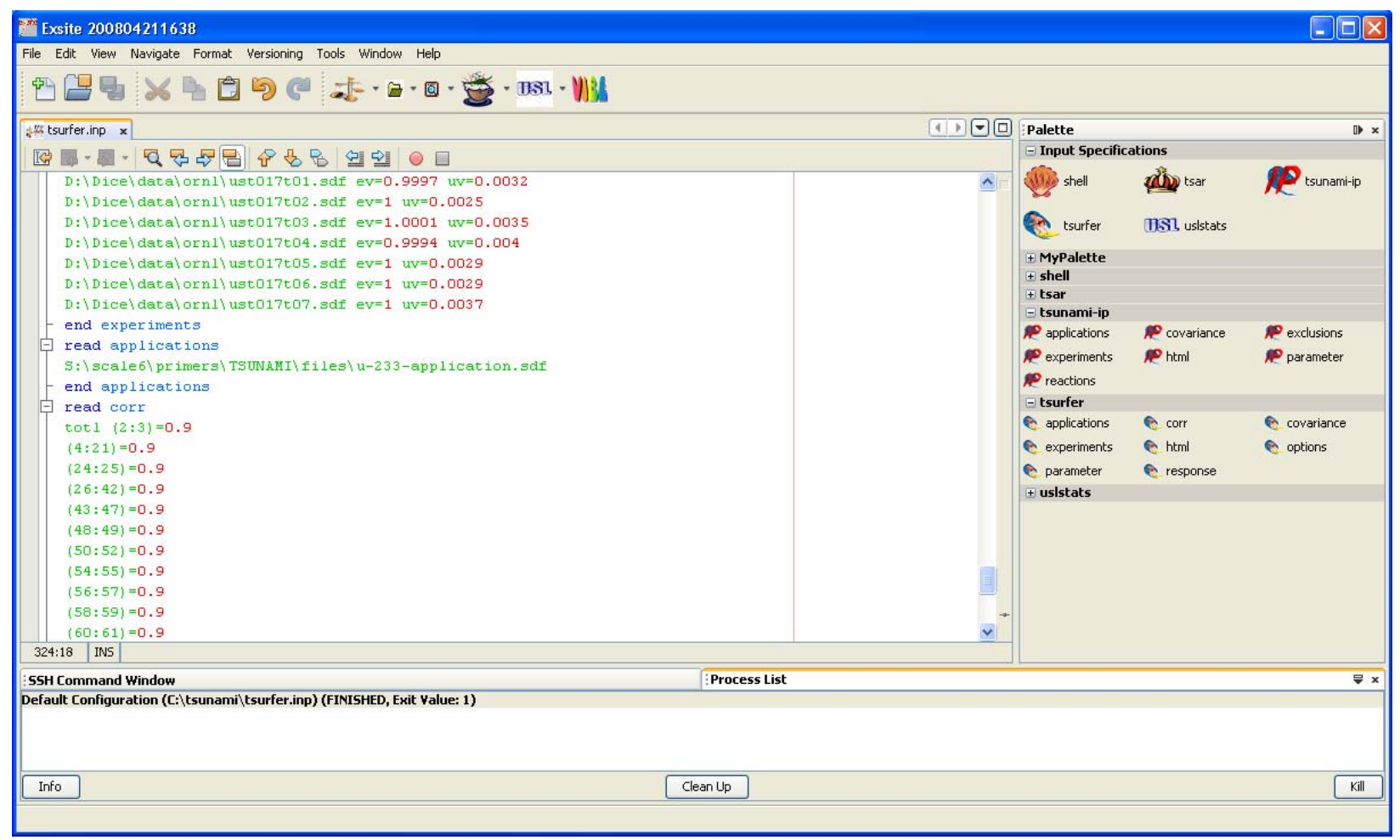

Fig. 181. TSURFER correlation data in ExSITE.

This input may require approximately $30 \mathrm{~min}$ to execute. When the execution is complete, open the HTML output file using the button on the ExSITE toolbar.

The TSURFER HTML output is arranged similarly to the TSUNAMI-IP HTML output, as shown by the program verification page in Fig. 182. The warning messages page includes the same covariance warnings as TSUNAMI-IP, but it also includes an additional message regarding the corr data block. Here it notes that the correlation coefficient between an experiment and itself must be 1.0. In the cor $r$ block, a value of 0.9 was assigned to some experiments' self-correlation, referred to as the diagonal element of the correlation matrix. TSURFER automatically corrects these diagonal terms to provide for shorter user input.

Many of the entries in the Input Data submenu are similar to those from TSUNAMI-IP or are self-explanatory. The input response list, shown in Fig. 184, lists all SDFs whether they are applications, experiments, or tagged to be omitted from the calculation. The uses of these SDFs are identified in the USE column as expt, appl, or omit, respectively. Also, the calculated and experimental values are listed. 


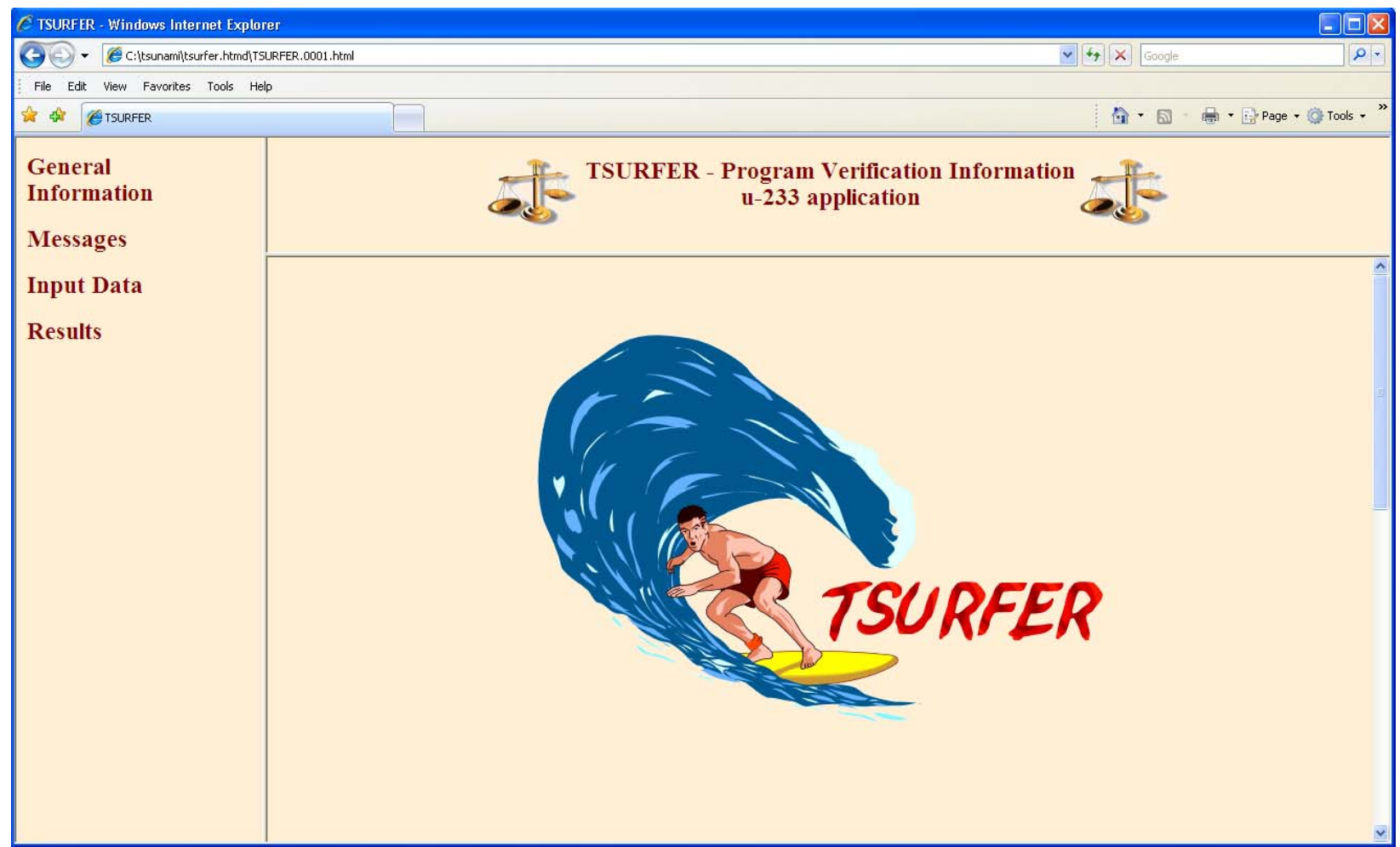

Fig. 182. TSURFER program verification page in HTML output.

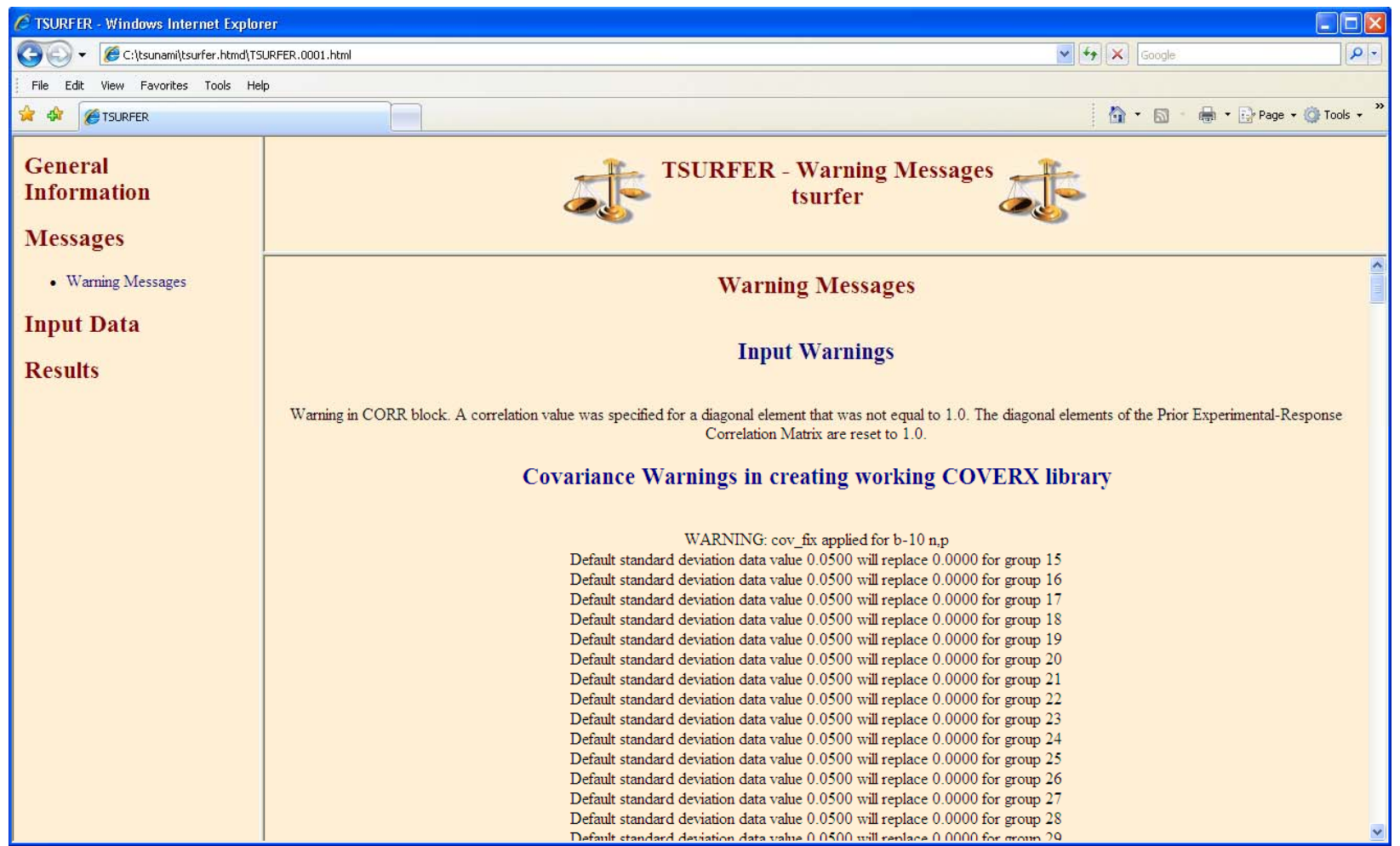

Fig. 183. TSURFER warning message in HTML output. 


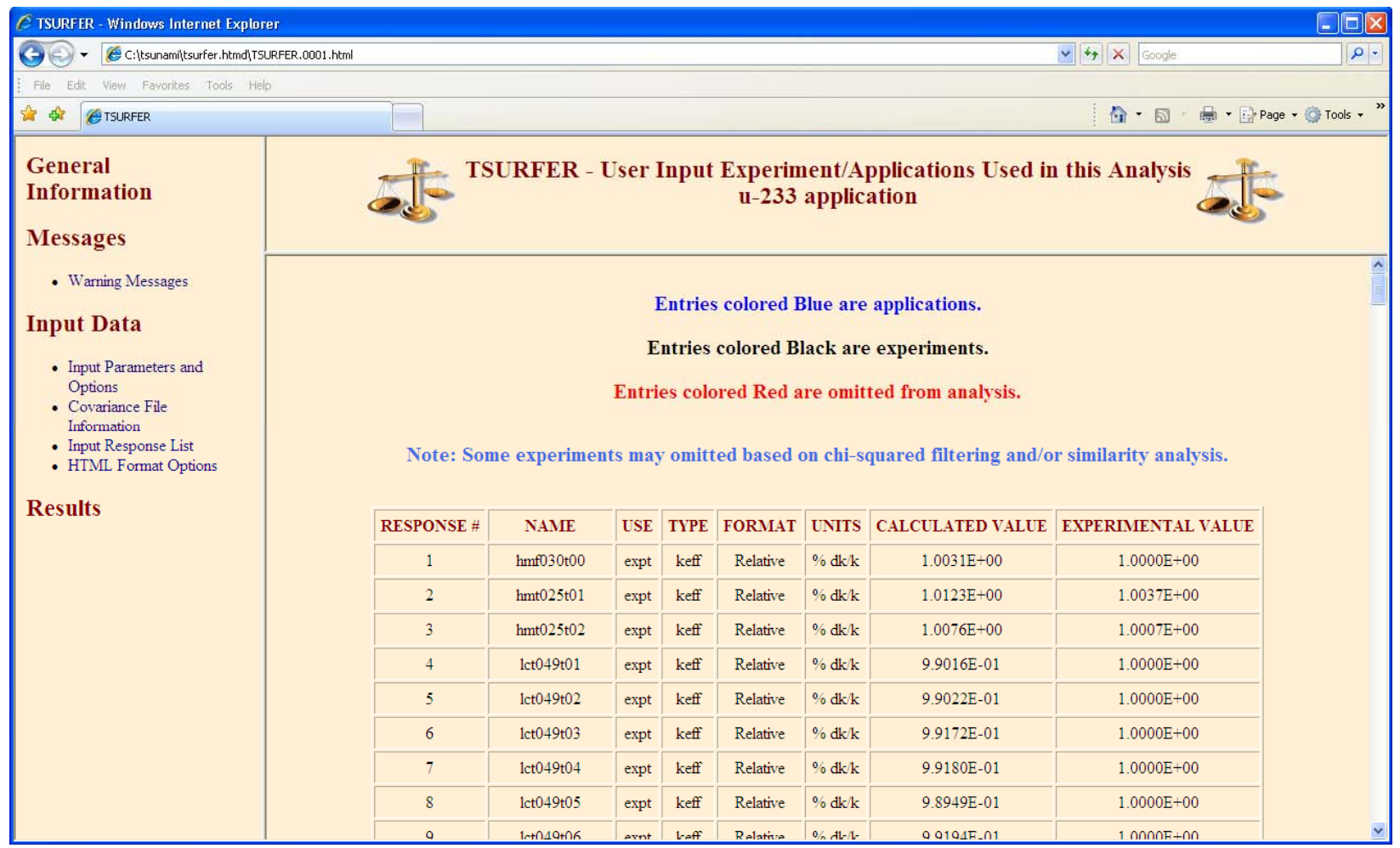

Fig. 184. TSURFER input response list in HTML output.

Under the Results submenu, the Application and Bias Summary edit, shown in Fig. 185, lists all applications (here only the ${ }^{233} \mathrm{U}$ application is included) in an edit that includes the original $k_{\text {eff }}$ as Calculated Value, the original uncertainty in $k_{\text {eff }}$ due to cross-section uncertainties as Prior Std Dev (\%), the relative bias in the application computed through the TSURFER data adjustment procedure as Relative Bias (\%), the absolute bias as Bias, the $k_{\text {eff }}$ value that would be computed if the adjusted cross sections were used as Adjusted Value, and the uncertainty in this adjusted $k_{e f f}$ value if the adjusted cross-section-covariance data were used as Adjusted Std Dev (\%).

In this case the bias computed with TSURFER is $0.259 \% \Delta \mathrm{k} / \mathrm{k}$, and the bias uncertainty, in terms of a single standard deviation of the adjusted results, is $0.0785 \% \Delta \mathrm{k} / \mathrm{k}$. Note that the bias uncertainty is defined as the uncertainty in the adjusted $k_{\text {eff }}$ value. The reduction in this uncertainty relative to the original uncertainty in the application, $1.0666 \% \Delta \mathrm{k} / \mathrm{k}$, is due to inclusion of additional information, the critical experiments, in the validation process. Here the uncertainty is reduced to approximately $8 \%$ of its original value, confirming that the benchmarks selected lead to adjustments in the cross sections that are the largest sources of uncertainty, and thus the largest potential sources of computational bias, for this application.

Further examining the results for this application, the individual sources of bias can be identified according to the impact of the adjusted cross section on the application's $k_{\text {eff }}$ value. TSURFER ranks the bias contributions according to an L1 norm, which is based on the absolute values of the groupwise cross-section adjustments. The top contributors to bias for the ${ }^{233} \mathrm{U}$ application are shown in Fig. 185. Comparing the TSURFER-predicted contribution to bias with the top sources of uncertainty shown in Fig. 124, one finds that the ${ }^{233} \mathrm{U}$ reactions and ${ }^{16} \mathrm{O}$ elastic rank at top of both tables. However, ${ }^{1} \mathrm{H}$ elastic scattering, the second highest source of uncertainty, appears as the sixth highest source of bias. For all 
nuclides and reactions, the bias contribution is approximately the same or less than the uncertainty shown in Fig. 124, as the adjustments in the cross sections are bounded with a $\chi^{2}$ of 1.2.

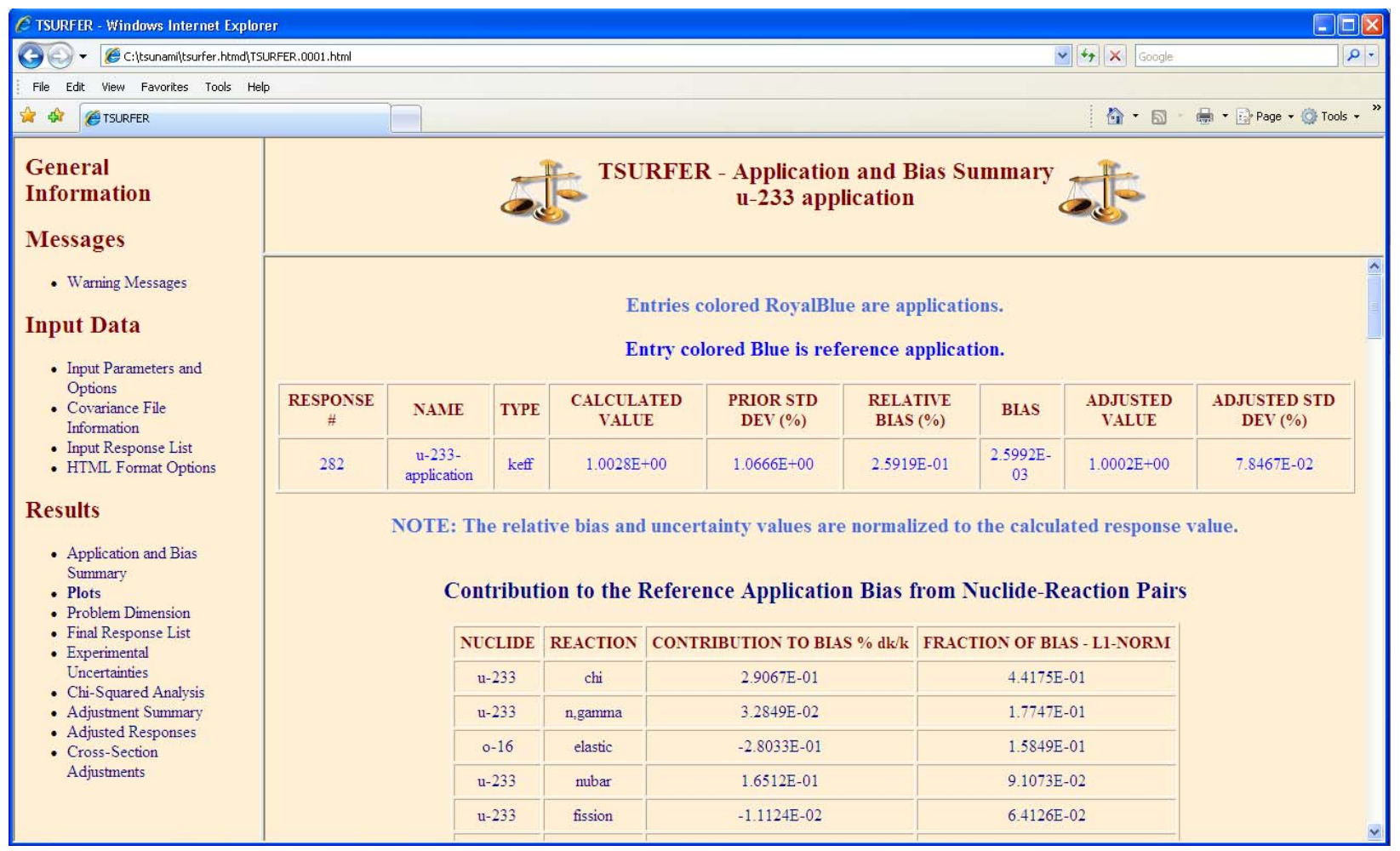

Fig. 185. TSURFER application and bias summary in HTML output.

The Adjustment Summary edit, shown in Fig. 186, details the impact of the data adjustments on each experiment. An overall consistency should be achieved between the calculated and experimental values after the adjustment procedure. Where an experiment must be adjusted too far outside its uncertainties to achieve consistency between its calculated and experimental values, the experiment is omitted from the calculation. The $\chi^{2}$ filter is used to achieve this consistency. The input parameter target_chi sets the level of desired consistency, and chi_sq_filter sets the method for filtering experiments. These techniques are described in more detail in the TSURFER manual. In Fig. 186, two experiments shown were omitted by the $\chi^{2}$ filtering procedure to achieve the default target value of 1.2 using the default delta_chi technique.

In this calculation, 100 experiments were omitted by the stringent default $\chi^{2}$ criteria, as shown in the Problem Dimension edit in Fig. 187. 


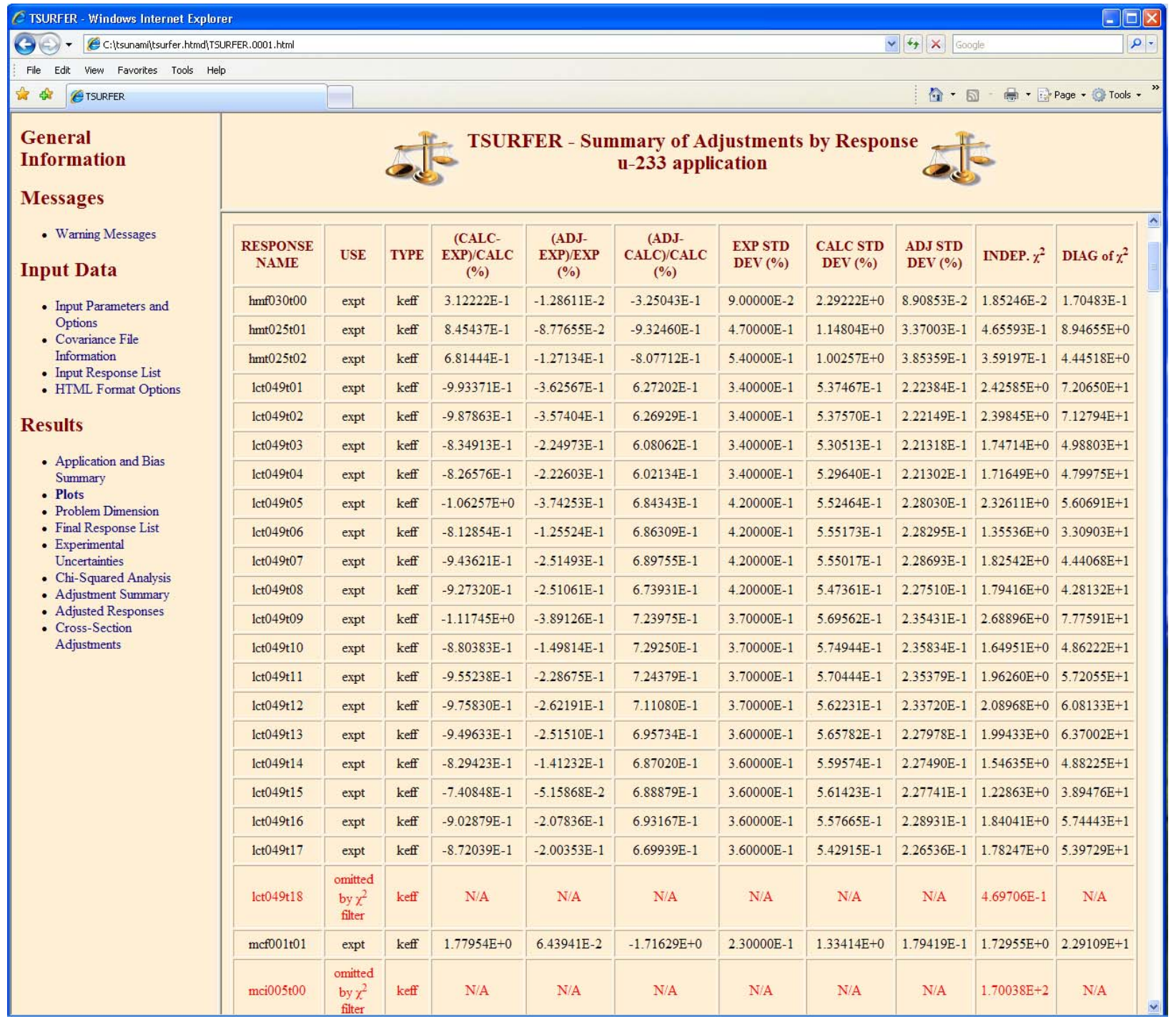

Fig. 186. TSURFER adjustment summary in HTML output. 


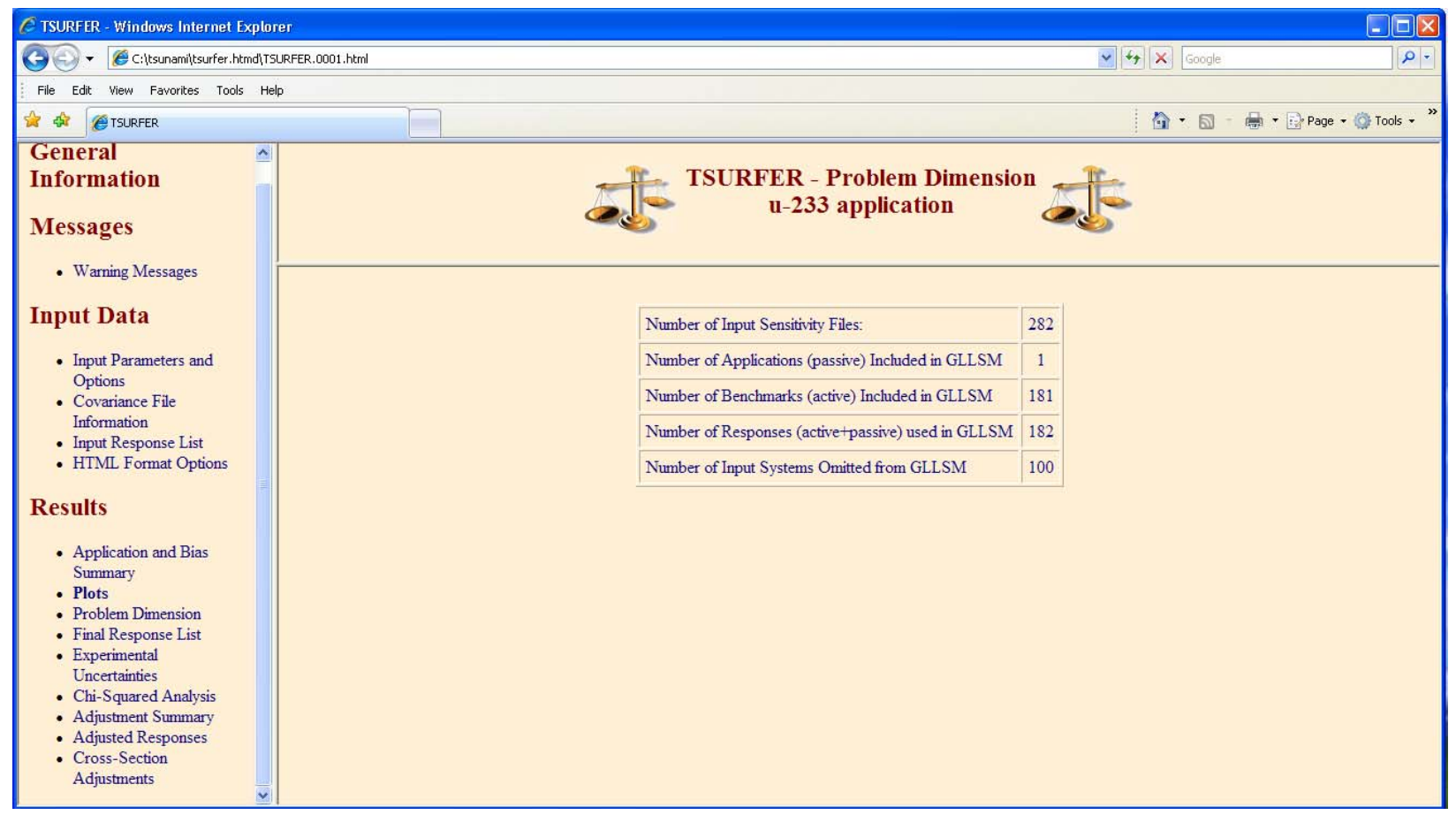

Fig. 187. TSURFER problem dimension edit in HTML output.

Because the input parameter print_adjustments was entered, the details of the individual cross-section adjustments can be seen in the Cross-Section Adjustments edit shown in Fig. 188. Here the percent changes in the individual groupwise cross sections, in the SCALE 44 group structure, are shown along with the uncertainty in the cross section before, (Prior), and after, (Adjusted), the adjustment procedure. The sensitivity coefficients for the reference application are shown, and the impact of the adjustment on the application bias is given for each group as both a relative contribution and a contribution to the L1 norm.

The nuclide-reaction-dependent output tables are selected with the menu on the right side of the browser, where the nuclide-reaction pairs are listed in order of descending contribution to the bias of the reference application. Viewing the ${ }^{233} \mathrm{U}$ chi table, the group with the largest adjustment is group 1; however, the largest contribution to bias, the product of the adjustment and the sensitivity of $k_{\text {eff }}$ to the adjustment, is observed for group 10. 


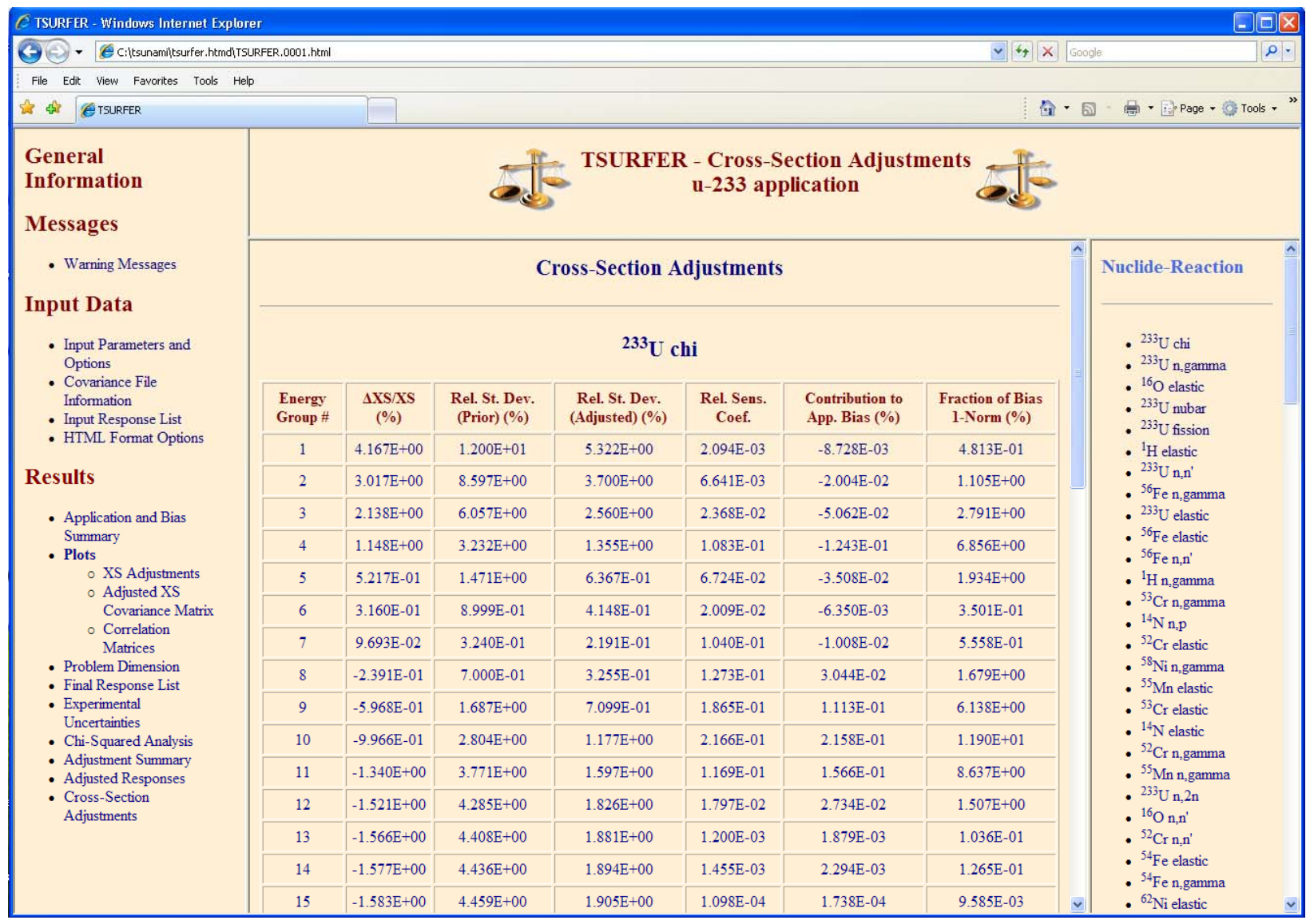

Fig. 188. TSURFER cross-section adjustments in HTML output.

Under the Plots submenu, the XS Adjustments plot provides a means of visualizing the relative changes in the cross sections using the applet version of Javapeño. A plot of the cross-section adjustments for the top five contributors to bias for the ${ }^{233} \mathrm{U}$ application is given in Fig. 189. A maximum positive adjustment of $\sim 7.5 \%$ is observed for ${ }^{233} \mathrm{U}$ fission, and a maximum negative adjustment of $\sim 8.0 \%$ is observed for ${ }^{233} \mathrm{U}$ n,gamma.

Because this input file was called tsurfer . inp, a Javapeño-formatted plot file is returned to the user directory with the name tsurfer.xs-adjust.plt. In a version of Javapeño that will be released after SCALE 6, the energy-dependent cross-section adjustments can be multiplied by the energydependent sensitivity of the application's $k_{\text {eff }}$ to the cross sections to form an energy-dependent bias resulting from a specific nuclide-reaction pair. As shown in Fig. 190, the application bias is dependent on which cross sections were modified in the adjustment procedure and how sensitive the application is to those adjustments. For example, the maximum adjustment for ${ }^{233} \mathrm{U}$ fission occurs around $20 \mathrm{keV}$. However, the application is not very sensitive to the cross section in this energy range, and the bias due to this adjustment is small. In the intermediate energies, the adjustment is on the order of $1 \%$, but the application is more sensitive at these energies, so a larger bias contribution results. Also, as the thermaland fast-energy cross sections are reduced where the intermediate-energy cross sections are increased, a negative contribution to the bias is observed for thermal and fast, energies and a positive contribution to the bias is observed for intermediate energies. 


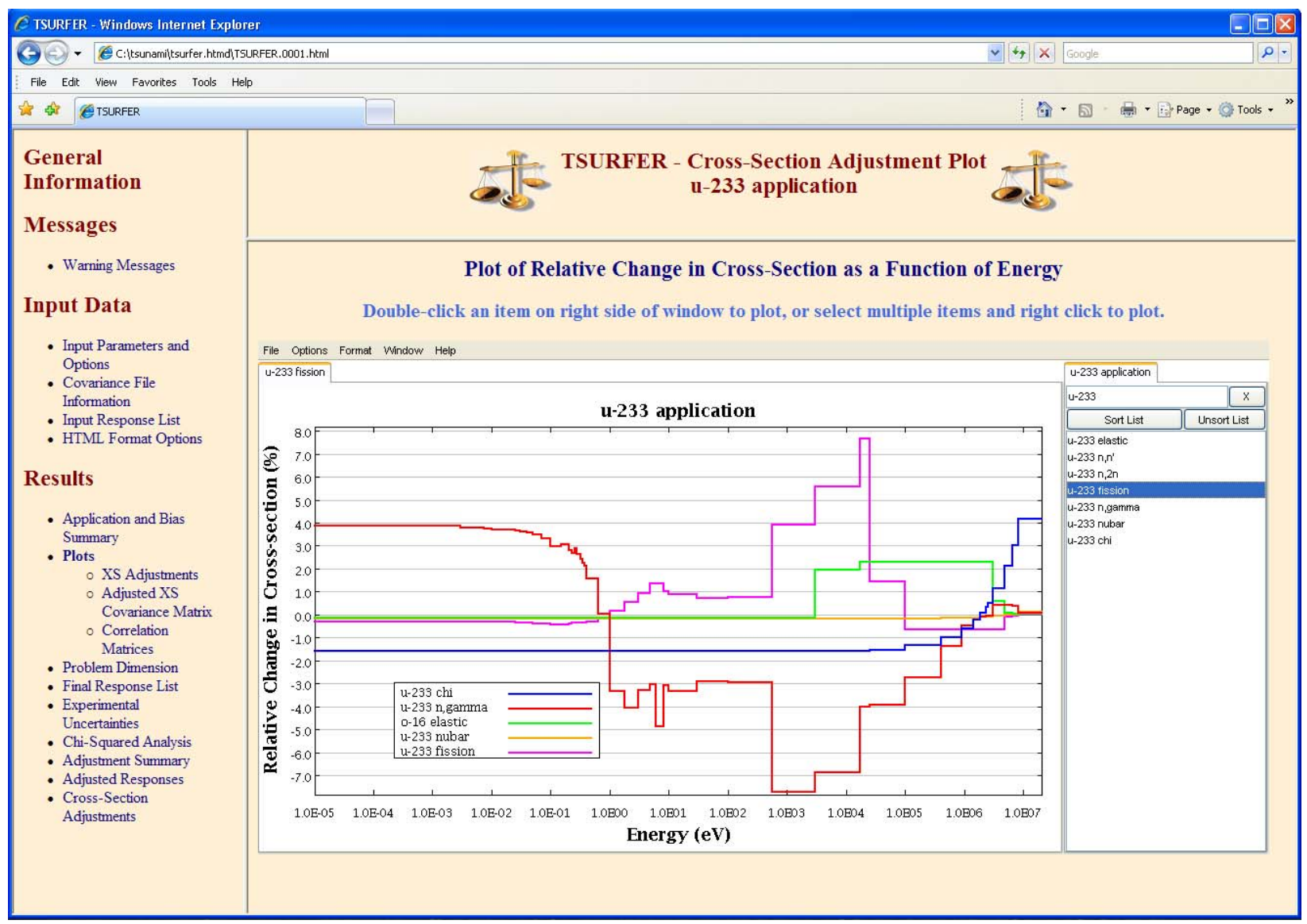

Fig. 189. TSURFER cross-section adjustment plot in HTML output. 


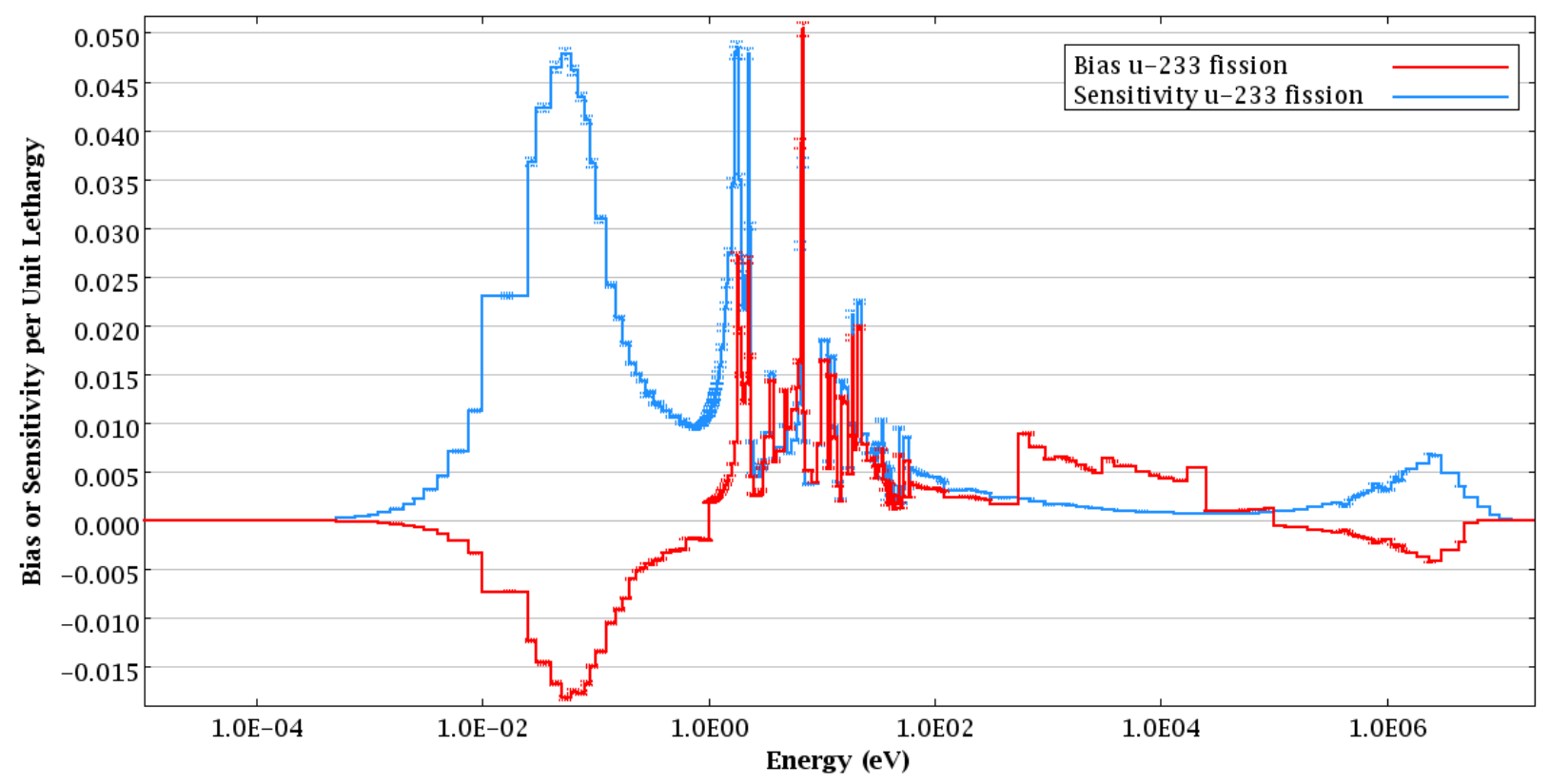

Fig. 190. Plot of energy-dependent bias and sensitivity for ${ }^{233} U$ fission for ${ }^{233} U$ application.

Because the TSURFER bias uncertainty is simply the uncertainty in the adjusted, or unbiased, cross-section data, the adjusted covariance matrix resulting from the TSURFER procedure can be used to provide contributions to the bias uncertainty by individual covariance matrices using the extended uncertainty edit of TSUNAMI-IP. Similar to the extended penalty assessment from Sect. 9.2, an extended uncertainty assessment with a postadjustment covariance matrix reveals components in the application that are not validated with the selected benchmarks. Because the input file is called tsurfer. inp and return_adj_cov was entered in the TSURFER parameter input, the postadjustment covariance library is returned to the user's directory as tsurfer.adj.cov. The SCALE "shell" module can be used to copy the adjusted covariance data to the SCALE temporary directory, as shown at the top of the input in Fig. 191. The short TSUNAMI-IP input shown in Fig. 191 can be use to compute the uncertainties of the ${ }^{233} \mathrm{U}$ application that remain after the adjustment procedure. 


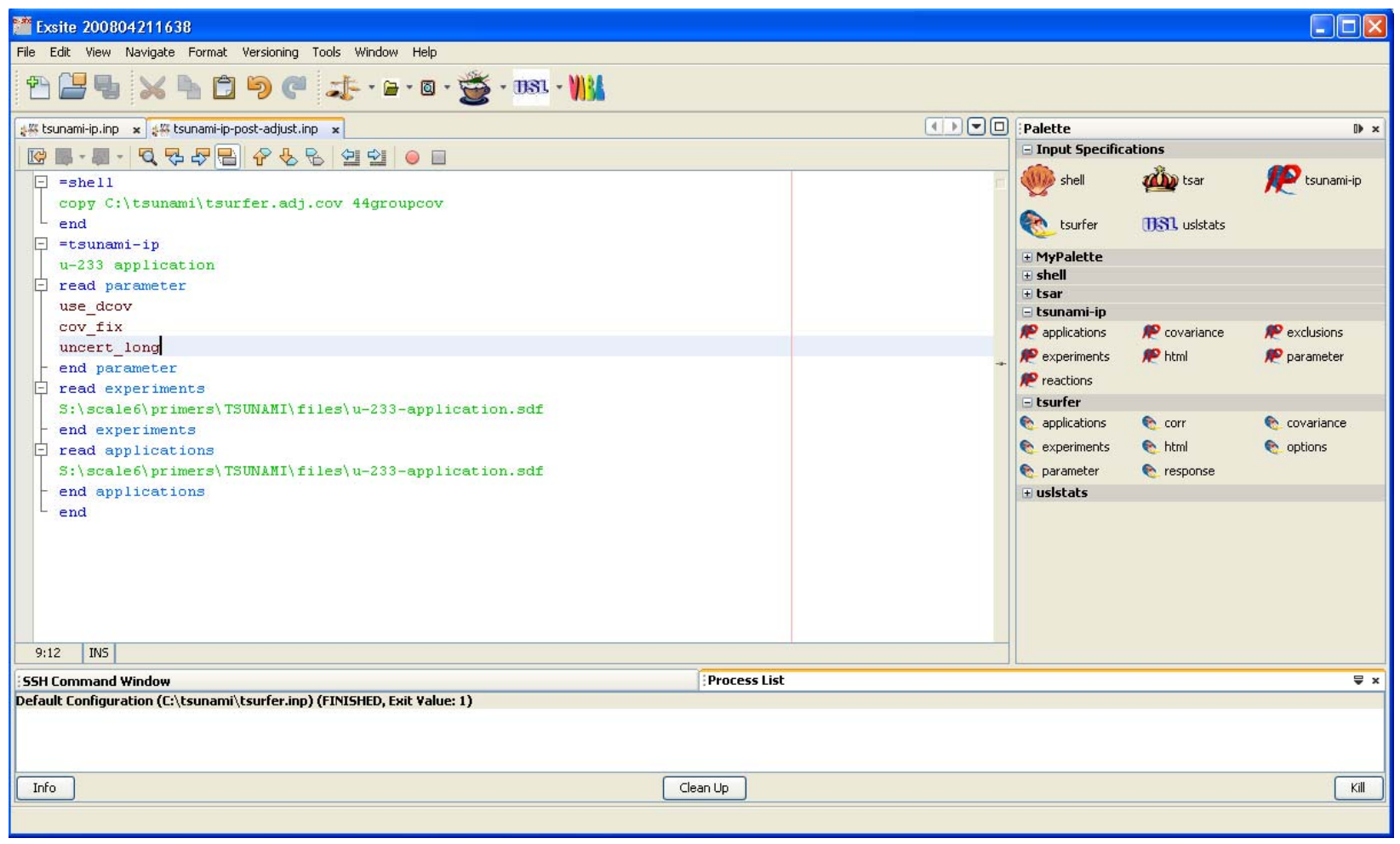

Fig. 191. ExSITE with TSUNAMI-IP input to compute postadjustment uncertainties.

The contributions to the uncertainty in the bias are shown in the TSUNAMI-IP extended uncertainty edit in Fig. 192. Here anticorrelations in the adjusted covariance data become important components in the reduced uncertainty. Because all cross sections are adjusted from the same set of benchmark experiments, the number of cross correlations in uncertainties for different nuclides and reactions greatly increases. Where sufficient quality benchmarks are available to validate a given nuclide reaction, the uncertainty in that nuclide reaction is reduced. However, due to correlations introduced in the adjustment procedure, the uncertainty in the cross section may be increased if only limited benchmarks that have significant sensitivities to a given nuclide reaction are available. 


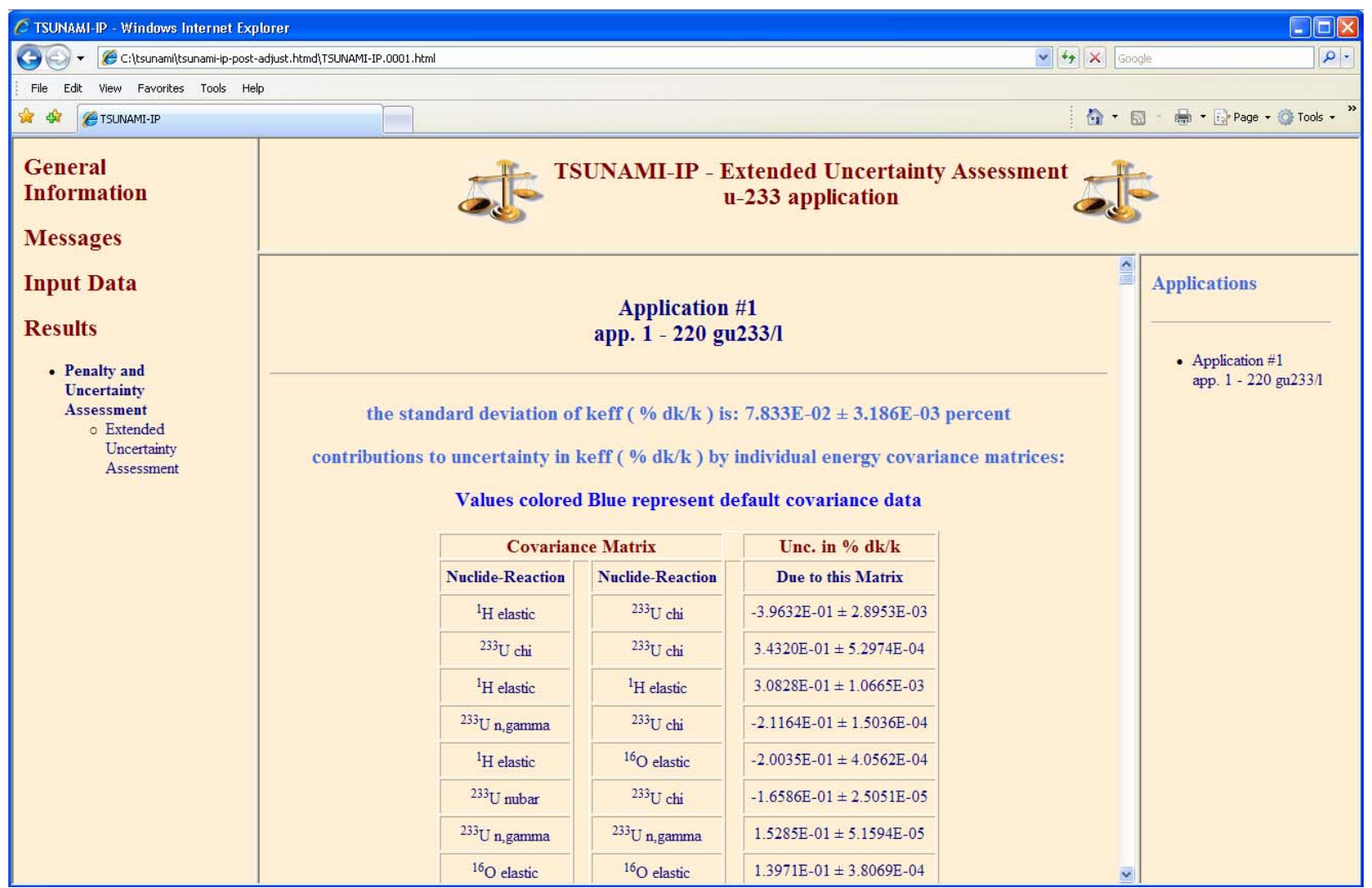

Fig. 192. TSUNAMI-IP extended uncertainty edit using postadjustment covariance data.

In comparing the results shown in Fig. 192 to the original uncertainties before the adjustment, shown in Fig. 124, the uncertainties for all nuclide-reaction pairs are reduced, and new cross correlations are introduced, further reducing the uncertainty. 


\section{CONCLUSIONS}

This primer has presented examples in the application of the SCALE/TSUNAMI tools to generate $k_{\text {eff }}$ sensitivity data for 1D and 3D models using TSUNAMI-1D and -3D and to examine uncertainties in the computed $k_{\text {eff }}$ values due to uncertainties in the cross-section data used in their calculation. The proper use of unit cell data and need for confirming the appropriate selection of input parameters through direct perturbations were described.

The uses of sensitivity and uncertainty data to identify and rank potential sources of computational bias in an application system and TSUNAMI tools for assessment of system similarity using sensitivity and uncertainty criteria were demonstrated. Uses of these criteria in trending analyses to assess computational biases, bias uncertainties, and gap analyses were also described. Additionally, an application of the data adjustment tool TSURFER was provided, in which specific details of sources of computational bias were identified.

These examples do not provide an exhaustive review of all possible applications of the TSUNAMI tools. However, it is hoped that they provide the interested reader with enough information to apply the TSUNAMI tools of SCALE 6 in practical analyses.

Those wishing to learn about the TSUNAMI techniques are encouraged to attend a TSUNAMI training course, typically offered twice a year by the SCALE staff at ORNL. 



\section{REFERENCES}

1. SCALE: A Modular Code System for Performing Standardized Computer Analyses for Licensing Evaluation, ORNL/TM-2005/39, Version 5.1, Vols. I-III, Oak Ridge National Laboratory, Oak Ridge, Tenn., November 2006. Available from Radiation Safety Information Computational Center at Oak Ridge National Laboratory as CCC-732.

2. MCNP-A General Monte Carlo N Particle Transport Code, Version 5, Vols. I-III, Oak Ridge National Laboratory, Oak Ridge, Tenn., February 2008. Available from Radiation Safety Information Computational Center at Oak Ridge National Laboratory as CCC-740.

3. American National Standard for Nuclear Criticality Safety in Operations with Fissionable Materials Outside Reactors, ANSI/ANS-8.1-1998, American Nuclear Society, La Grange Park, Ill., 1998.

4. American National Standard for Validation of Neutron Transport Methods for Nuclear Criticality Safety Calculations, ANSI/ANS-8.24-2007, American Nuclear Society, La Grange Park, Ill., 2007.

5. J. J. Lichtenwalter et al., Criticality Benchmark Guide for Light-Water-Reactor Fuel in Transportation and Storage Packages, NUREG/CR-6361 (ORNL/TM-13211), Oak Ridge National Laboratory, Oak Ridge, Tenn., 1997.

6. B. L. Broadhead et al., "Sensitivity- and Uncertainty-Based Criticality Safety Validation Techniques," Nucl. Sci. Eng. 146, 340-366, 2004.

7. P. R. Bevington, Data Reduction and Error Analysis for the Physical Sciences, McGraw-Hill Book Company, New York, 1969.

8. R. D. Busch and S. M. Bowman, KENO V.a Primer: A Primer for Criticality Calculations with SCALE/KENO V.a Using GeeWiz, ORNL/TM-2005/135, Oak Ridge National Laboratory, Oak Ridge, Tenn., January 2005.

9. D. E. Mueller, B. T. Rearden, and D. F. Hollenbach, Application of the SCALE TSUNAMI Tools for the Validation of Criticality Safety Calculations Involving ${ }^{233}$ U, ORNL/TM-2008/196, Oak Ridge National Laboratory, Oak Ridge, Tenn. January 2009.

10. D. E. Mueller and G. A. Harms, "Using the SCALE 5 TSUNAMI-3D Sequence in Critical Experiment Design,” Trans. Am. Nucl. Soc. 93, 263-266 (2005).

11. J. C. Dean and R. W. Tayloe, Jr., Guide for Validation of Nuclear Criticality Safety Calculation Methodology, NUREG/CR-6698, U. S. Nuclear Regulatory Commission, Science Applications International Corporation, January 2001.

12. V. R. Cain, A Computer Code to Perform Analyses of Criticality Validation Results, Y/DD-574, Martin Marietta Energy Systems, Inc., Oak Ridge Y-12 Plant, Oak Ridge, Tenn., September 1995. 
